\title{
Effects of drought on the growth of young and mature temperate forests in West Virginia
}

Nicholas R. Doner

West Virginia University

Follow this and additional works at: https://researchrepository.wvu.edu/etd

\section{Recommended Citation}

Doner, Nicholas R., "Effects of drought on the growth of young and mature temperate forests in West Virginia" (2004). Graduate Theses, Dissertations, and Problem Reports. 877.

https://researchrepository.wvu.edu/etd/877

This Thesis is protected by copyright and/or related rights. It has been brought to you by the The Research Repository @ WVU with permission from the rights-holder(s). You are free to use this Thesis in any way that is permitted by the copyright and related rights legislation that applies to your use. For other uses you must obtain permission from the rights-holder(s) directly, unless additional rights are indicated by a Creative Commons license in the record and/ or on the work itself. This Thesis has been accepted for inclusion in WVU Graduate Theses, Dissertations, and Problem Reports collection by an authorized administrator of The Research Repository @ WVU. For more information, please contact researchrepository@mail.wvu.edu. 
Effects of Drought on the Growth of Young and

Mature Temperate Forests in West Virginia

Nicholas R. Doner

Thesis Submitted to the College of Arts and Sciences

at West Virginia University

in Partial Fulfillment of the Requirements for

the Degree of

Master of Arts in Geography

Amy E. Hessl, Ph.D., Chair

William T. Peterjohn, Ph.D.

Timothy A. Warner, Ph.D.

Department of Geology and Geography

Morgantown, West Virginia

2004

Keywords: Fernow Experimental Forest, West Virginia, Temperate Deciduous Forests, Aboveground Net Primary Productivity, Drought 


\section{ABSTRACT \\ Effects of Drought on the Growth of Young and Mature Temperate Forests in West Virginia}

Nicholas R. Doner

Recent research suggests that the temperate deciduous forests of the eastern United States may have played a significant role in the sequestration of atmospheric carbon dioxide over the last 100 years. However, little is known about the role of forest management and climatic variability on the productivity of these forests. The purpose of this study was to determine the variation in aboveground production between two different aged forest stands and to evaluate the effect of drought on inter-annual variation in productivity. Aboveground net primary production (ANPP) was compared with precipitation and the Palmer Drought Severity Index to determine the effects of drought on aboveground productivity. I found that: 1) there was a significant difference in rates of ANPP between the two watersheds due to age; 2) drought conditions reduce the rate of aboveground productivity; and 3) the reduction of aboveground productivity was significantly related to species-specific factors; thus the productivity of some temperate species may be affected more by drought than others. 


\section{ACKNOWLEDGMENTS}

The author gratefully acknowledges the guidance, patience, and support given him by Dr. Amy E. Hessl (Department of Geology and Geography) during his term of study. Her assistance in the field and in the laboratory throughout the production of this thesis will be long remembered. In addition, the author also wishes to recognize the other members of his graduate committee, Dr. William T. Peterjohn (Department of Biology) and Dr. Timothy A. Warner (Department of Geology and Geography) for their time and advice.

The author also gratefully acknowledges the several undergraduates (Jodie Hoover, Matthew Perkowski, Jon Michael Bosley, and Aimee Phoenix) who provided their time and expertise with the field and laboratory work, as well as Dr. Stanley Wearden (Department of Statistics) and Dr. John Brooks (Department of Forestry) who provided comments, suggestions, and insights, and Paige Eagle (University of Washington) for her development of the net primary productivity calculator.

Finally, the author wishes to acknowledge the encouragement and support of his parents and younger sister. 


\section{TABLE OF CONTENTS}

$\underline{\text { Page }}$

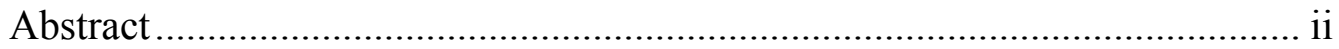

Acknowledgements ................................................................................

Table of Contents ............................................................................... iv

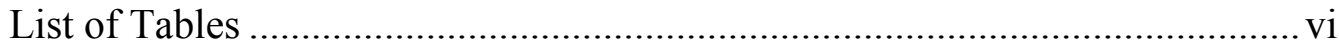

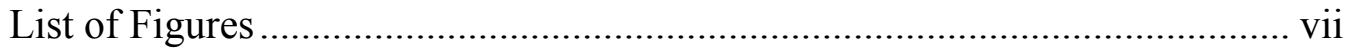

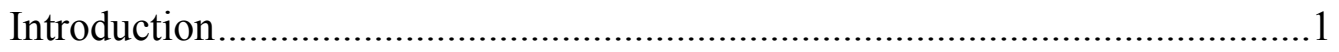

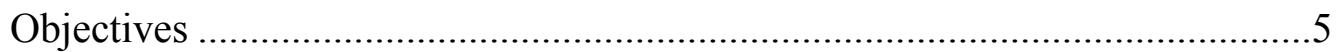

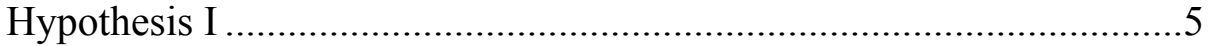

Hypothesis II .............................................................................

Hypothesis III...........................................................................

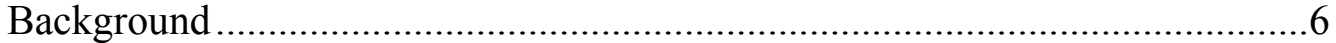

Measuring Growth (Aboveground Net Primary Production/

Carbon Sequestration) in Forests ...................................................6

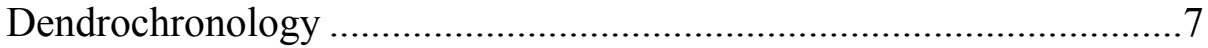

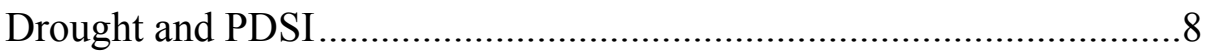

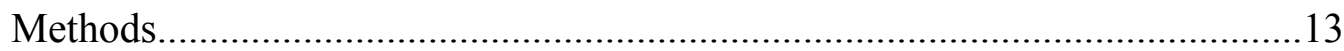

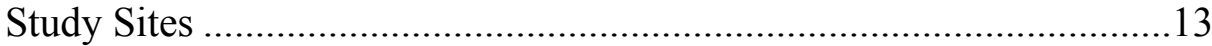

Field Methods .................................................................................15

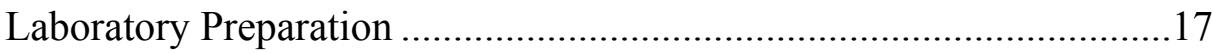

Biomass/Net Primary Productivity Calculations ................................18

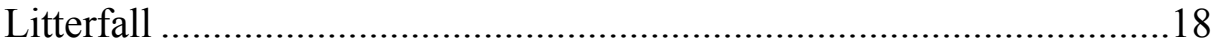

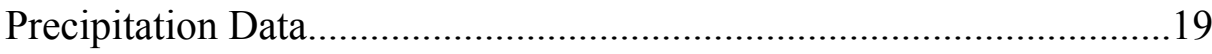

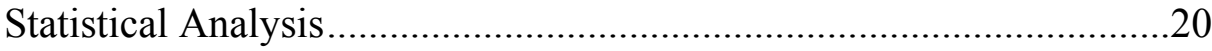

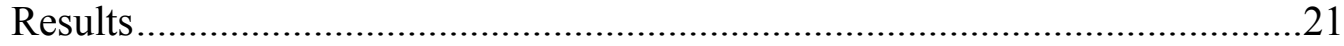

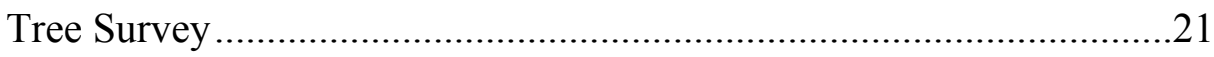

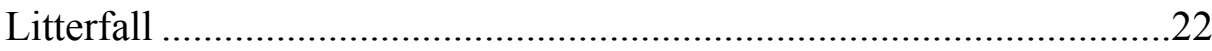

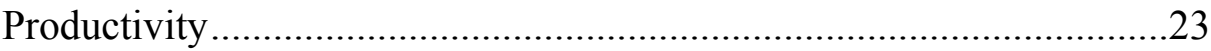

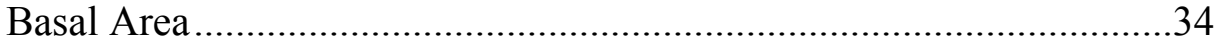




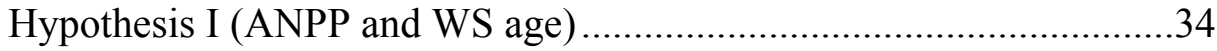

Hypothesis II (ANPP, WS age, and drought) .......................................34

Hypothesis III (ANPP, tree species, and drought)..................................35

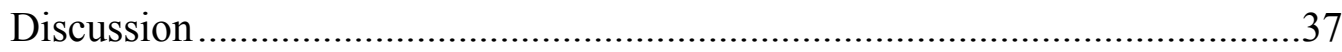

References Cited ......................................................................................4

Appendix A (Tree Species and Abbreviations) ...............................................49

Appendix B (Tree Biomass Allometric Equations) .............................................50

Appendix C (Tree Species Frequency per Plot) ..................................................51

Appendix D (Litterfall Weights (g) per Species per Plot) ..................................52

Appendix E (Bark Coefficients) .....................................................................53

Appendix F (Tree Survey and Measurements) ……………………………....54

Appendix G (Increment Core Measurements) ......................................................59

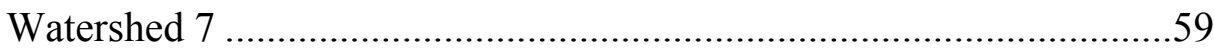

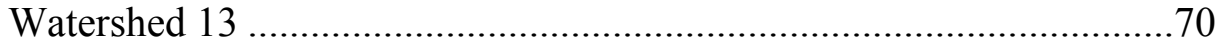

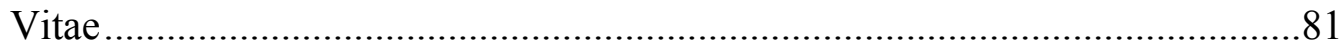




\section{LIST OF TABLES}

$\underline{\text { Page }}$

Table 1: Palmer classes for wet and dry periods.........................................11

Table 2: Physical and ecological characteristics of watershed 7 and watershed 13 (Adams et al., 1994)..........................13

Table 3: Aspect, slope, and elevation position

for the 12 plots in watershed 7 and watershed 13

Table 4: Tree species sampled in watershed 7 and watershed $13 \ldots \ldots \ldots \ldots \ldots \ldots . . .21$

Table 5: Summary of ring width measurements for watershed 7 and watershed 13 expressed in $\mathrm{cm}$

Table 6: Summary of litterfall weight for the Fernow

from 1991-2002 for selected collection baskets in watershed 7, expressed in $\mathrm{kg} / \mathrm{ha} /$ year (a), and for 2002 in newly established collection baskets in watershed 7 and watershed 13, expressed in $\mathrm{kg} / \mathrm{ha} /$ year (b)

Table 7: Mean ANPP excluding litterfall (1971-2001)

expressed in $\mathrm{kg} / \mathrm{ha} / \mathrm{year}$, and biomass (2002)

for plots in watershed 7 and watershed 13

expressed in $\mathrm{kg} / \mathrm{ha}$

Table 8: Pearson correlation coefficients (r) for the annual average ANPP for each plot and annual precipitation, growing season precipitation, and PDSI values for the 1971-1995 time period (a), and for the 1957-2000 time period (b)

Table 9: Basal area for each plot and watershed average $\left(\mathrm{cm}^{2} / \mathrm{ha}\right)$..................34

Table 10: ANOVA and ANCOVA results for each of the three hypotheses examined 


\section{LIST OF FIGURES}

Figure 1: The short-term reservoirs and movement of carbon through the biosphere and atmosphere. 1

Figure 2: Fernow Experimental Forest total annual precipitation (1970-2000) with full-drought (red diamonds), partial-drought (green diamonds), and non-drought (gray diamonds) years plotted

Figure 3: Fernow Experimental Forest growing season (May-September) precipitation (1970-2000) with full-drought (red diamonds), partial-drought (green diamonds), and non-drought (gray diamonds) years plotted

Figure 4: 1970-1995 PDSI values (grid point135) 12

Figure 5: Locations of watershed 7 (WS 7) and watershed 13 (WS 13) in relation to one another and other watersheds at the Fernow Experimental Forest, Parsons, West Virginia

Figure 6: Location of the 12 study plots in watershed 7 and watershed 13 (distances expressed in UTM)

Figure 7: Box plots of plot average ANPP excluding litterfall $(\mathrm{kg} / \mathrm{ha} /$ year $)$ for watershed 7 and watershed 13 for 1971-2001, with the middle line showing the median....

Figure 8: Box plots of plot average biomass ( $\mathrm{kg} / \mathrm{ha})$ for watershed 7 and watershed 13 for 2002 , with the middle line showing the median.

Figure 9: ANPP excluding litterfall (kg/ha/year) for watershed 7 (1971-2001) with diamonds showing estimated values, standard deviations bars; circles showing the estimated values including litterfall collected by the Fernow staff (WS 7) 
Figure 10: ANPP excluding litterfall $(\mathrm{kg} / \mathrm{ha} / \mathrm{year})$ for watershed 13 (1971-2001) with diamonds showing estimated values, standard deviations bars; circles showing the estimated values including litterfall collected by the Fernow staff (WS 7)

Figure 11: ANPP excluding litterfall $(\mathrm{kg} / \mathrm{ha} / \mathrm{year})$

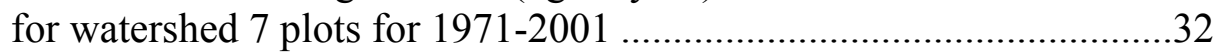

Figure 12: ANPP excluding litterfall $(\mathrm{kg} / \mathrm{ha} /$ year $)$ for watershed 13 plots for 1971-2001 .33 


\section{INTRODUCTION}

Atmospheric carbon dioxide concentrations have increased approximately $30 \%$ over the last 200 years due to the combustion of fossil fuels (Ciais et al., 2000; IPCC, 2001). However, atmospheric carbon dioxide is just one component of a complex carbon cycle, which includes living organisms, as well as dead organisms, carbonates, and fossil fuels (Schlesinger, 1997) (Figure 1).

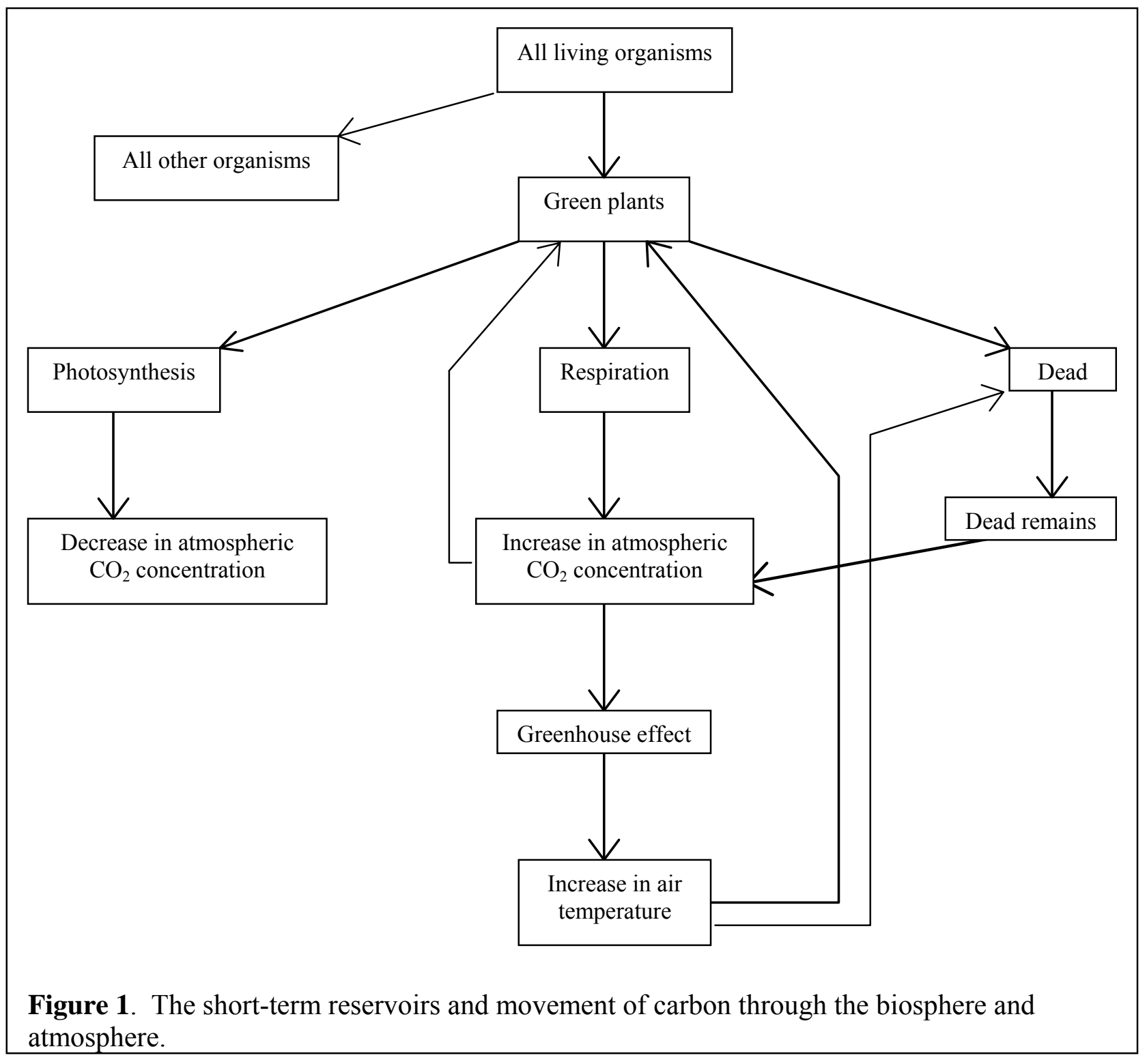


An important aspect of the carbon cycle is the fact that living organisms are partly composed of carbon. Through photosynthesis, trees and other plants remove carbon dioxide from the atmosphere, and as a result, have the potential to decrease the concentration of atmospheric carbon dioxide. Therefore, vegetation and especially forest biomes play an integral role in the sequestration of carbon, via carbon dioxide (DeLucia et al., 1999; Harmon, 2001). However, while plants can remove carbon dioxide from the atmosphere, they also release carbon dioxide back into the atmosphere through respiration, as well as decomposition via microbes. This in turn can enhance the greenhouse effect, potentially increasing air temperature and altering other climatic variables.

Terrestrial vegetation accounts for the third largest carbon pool $\left(5.6 \times 10^{17} \mathrm{~g} \mathrm{C} ; 1.4 \%\right.$ of total) of the total active global carbon cycle/budget $\left(\approx 4.0 \times 10^{19} \mathrm{~g} \mathrm{C}\right)$, with the atmospheric pool $\left(7.5 \times 10^{17} \mathrm{~g} \mathrm{C} ; 1.9 \%\right)$ and the oceanic pool $\left(3.8 \times 10^{19} \mathrm{~g} \mathrm{C} ; 95 \%\right)$ being larger (Schlesinger, 1997). Forests cover roughly $28 \%$ of Earth's terrestrial surface and have higher net primary production than other terrestrial vegetation types (Carey et al., 2001). However, forests have the potential to store more carbon than they already do through increasing the size of forested area and carefully selecting the management system(s) used (Harmon, 2001). Enhanced forest growth is important because it helps sequester carbon dioxide, thereby offsetting some of the emissions due to human activity (Moffat, 1998). Exploiting this potential is one of several proposed strategies to temporarily slow the increase of atmospheric carbon dioxide concentrations (Harmon, 2001).

The temperate deciduous forests of the eastern United States are the largest assemblage of deciduous tree species found anywhere in the world, and may be a significant carbon sink. Modeling of atmospheric carbon dioxide suggests that forests of the United States may play a 
disproportionate role in removing carbon from the atmosphere (Schimel et al., 2000; Goodale et al., 2002). Ciais et al. (2000) found that the largest biospheric sink $\left(2.1 \times 10^{15} \mathrm{~g} \mathrm{C} / \mathrm{yr}\right)$ is in the middle to high latitudes in the northern hemisphere. In addition, Ciais et al. (2000) indirectly determined the net carbon sink(s) over North America were 5-6 x $10^{14} \mathrm{~g} \mathrm{C} / \mathrm{yr}$. One explanation for this large sink lies in the temperate deciduous forests of the eastern U.S. that were clear-cut more than once in the last 300 years. Since the last major timbering period, eastern deciduous forests may have sequestered more atmospheric carbon dioxide due to re-growth (Goulden et al., 1996). In addition to long term increases in sequestration due to forest re-growth, inter-annual climatic variations on seasonal time scales may modify annual carbon dioxide exchange and capture (Goulden et al., 1996)

Many climatic factors may affect tree growth, and by extension carbon sequestration, in eastern deciduous forests. Persistent above average tree growth in old growth oak forests in the Blue Ridge Mountains appears to be associated with wetter than average conditions (Abrams et al. 1997). Similarly, increment growth was reduced by $23 \%$ due to drought in deciduous forests in Kentucky, with canopy trees more strongly affected than understory trees (Liu and Muller 1993). In southern Quebec, major growth declines in Acer saccharum are triggered by drought, but exacerbated by insects and disease (Payette et al. 1996). Prunus serotina, Quercus rubra, Fraxinus americana, and Liriodendron tulipifera, in the Fernow Experimental Forest, West Virginia, show positive growth responses to rainfall of prior summer, autumn, and current summer (Pan et al. 1997). In summary, both heavy precipitation and drought during and prior to the growing season may have major effects on radial growth. However, these studies focused on radial growth, and did not consider how radial growth translates into productivity or carbon storage. 
In addition to precipitation, tree age may influence rates of carbon sequestration in forests. Previous research concluded that old growth forests were carbon sources because: 1) tree growth declines with age and 2) respiration in older trees uses more photosynthate, therefore, trees cannot accumulate carbon as biomass (Odum, 1969; Odum, 1971; Carey et al., 2001). As a result, many believe that young forests are optimal for sequestering carbon because they are growing faster than older forests (Harmon, 2001). In addition, older forests have more dead trees and higher rates of decomposition; so, they should release more carbon than younger forests. While older forests do have more dead and decaying material, production tends to offset losses (Harmon 2001). Replacing old forests by young, fast-growing trees following clear-cutting could in fact, result in a net release of carbon into the atmosphere due to a carbon "legacy" coarse woody debris that continues to decay while young forests grow. Most of the work that has supported these conclusions is based on research in the Pacific Northwest, dominated by evergreen needleleaf forests with moist maritime climatic conditions (Cohen and Harmon, 1996; Law et al., 2001). Additional research is required to determine whether these conclusions apply to the moist deciduous broadleaf forests of the eastern United States. Additional research is also needed that addresses temporal, spatial, and climatic aspects of forest growth to develop a better understanding of NPP in the world's forests; ecosystems that play a major role in the global carbon budget (Clark et al., 2001). 


\section{OBJECTIVES}

The purpose of this study is to determine the differences in productivity by young and mature forests, the differences in productivity between major temperate deciduous tree species, and the effects of drought in the productivity of different aged temperate forests. I hypothesize the following:

Hypothesis I:

- $\quad \mathbf{H}_{\mathbf{0}}$ : Young forests will have higher rates of aboveground productivity than mature forests.

$\mathbf{H}_{1}$ : Mature forests will have higher rates of aboveground productivity than young forests.

Hypothesis II:

- $\mathbf{H}_{\mathbf{0}}$ : Drought event(s) will not affect the aboveground net primary production in different aged watersheds differentially.

$\mathbf{H}_{1}$ : Drought event(s) will reduce the aboveground net primary production in different aged watersheds differentially.

Hypothesis III:

- $\quad \mathbf{H}_{\mathbf{0}}$ : Drought will not affect the productivity of temperate tree species differently.

$\mathbf{H}_{1}$ : Drought will affect the productivity of temperate tree species differently, such that, species that prefer moist habitats will be affected more. 


\section{BACKGROUND}

Measuring Growth (Aboveground Net Primary Production/Carbon Sequestration) in Forests

Net primary production (NPP) is gross primary production minus plant respiration or the formation of plant tissues and reserve substances during a given period (Newbould, 1967). Thus,

$$
P_{n}=P_{g}-R
$$

where $P_{n}$ is the net primary production, $P_{g}$ is the gross primary production, and $R$ is plant respiration (Hall et al., 1993). Because of the difficulty in measuring biomass in forested vegetation, forest NPP cannot be directly measured. Instead several indirect measuring techniques have been developed in order to estimate the NPP of forested ecosystems. The sum of aboveground biomass increment and fine litterfall are considered equivalent to aboveground net primary productivity (ANPP). Quite often though, forest ANPP is underestimated because branchfall and leaf litter, or litterfall, is not taken into account in allometric equations (Clark et al., 2001).

Two widely accepted methods of sampling, increment cores and trunk discs, are used to obtain aboveground biomass increment measurements for individual trees. Increment cores, taken at breast height $(1.37 \mathrm{~m})$ yield annual rings that are measured and then converted to biomass increments via allometric equations (Phipps, 1985; Clark et al., 2001; Fritts, 2001). Alternatively, an entire disc or partial disc of the tree's trunk can be collected to measure annual increment growth (Newbould, 1967). If the tree is alive and the complete destruction of the tree is not wanted, or needed, a tree stump or a solid log from the remains of a nearby tree can be used to obtain a viable disc for measurement (Newbould, 1967; Stokes and Smiley, 1996). As 
with increment cores, the annual rings are measured and then converted to biomass increments via allometric equations (Clark et al., 2001; Fritts, 2001).

\section{$\underline{\text { Dendrochronology }}$}

In order to calculate yearly biomass and ANPP, it is crucial to correctly measure the size and characteristics of each tree ring. Dendrochronology is the science that deals with the dating and study of annual growth rings in wood (Schweingruber, 1993; Fritts, 2001). While many have restricted the term to dating events, the application of dendrochronology to biological, environmental, and climatic phenomena, such as tree growth, productivity, and drought, has become increasingly useful (Fritts, 2001). In general, tree ring widths respond to varying environmental conditions, including climate (Schweingruber, 1993; Fritts, 2001). Tree rings can be matched with the corresponding annual and seasonal climatic information, such as precipitation and temperature, in order to determine the effects of different climatic phenomena on tree growth (Fritts, 2001). Appalachian forests appear to be especially responsive to drought conditions. For example, Pinus strobus in the southern Appalachian Mountains responded to a severe drought period with a substantial decrease in annual radial increment (Clinton et al., 1997). Following drought episodes, trees in northern Virginia exhibited growth reductions lasting two to three years, with some species showing reductions lasting up to six years (Orwig and Abrams, 1997). Overall, Quercus alba was the only species that experienced statistically significant growth reductions associated with drought years. Other species studied (Liriodendron tulipifera, Carya glabra, Nyssa sylvatica, Pinus virginiana, and Quercus velutina) experienced growth reductions associated with drought years, but these variations in growth were not significant to the same degree. These results indicate that tree species in West Virginia may be 
responsive to drought and that the ANPP of West Virginia forests may also be similarly related to climatic variability.

\section{$\underline{\text { Drought and PDSI }}$}

A key factor affecting tree growth at a regional scale in the eastern United States is drought (Liu and Muller, 1993). Palmer defined a drought period as "an interval of time, generally of the order of months or years in duration, during which the actual moisture supply at a given time rather consistently falls short of the climatically expected or climatically appropriate moisture supply" (Palmer, 1965; Alley, 1984). It is important to remember that droughts are, by nature, regional phenomena (Alley, 1984). For the eastern region of the United States a deficit of $15 \%$ or greater over a six to twelve month period is considered a drought (Weather Forecast Office Pittsburgh, 2002). Several droughts of this magnitude have occurred in the last 30 years (Figures 2 and 3). 
Fernow Experimental Forest Precipitation Data (1970-2000) WS7

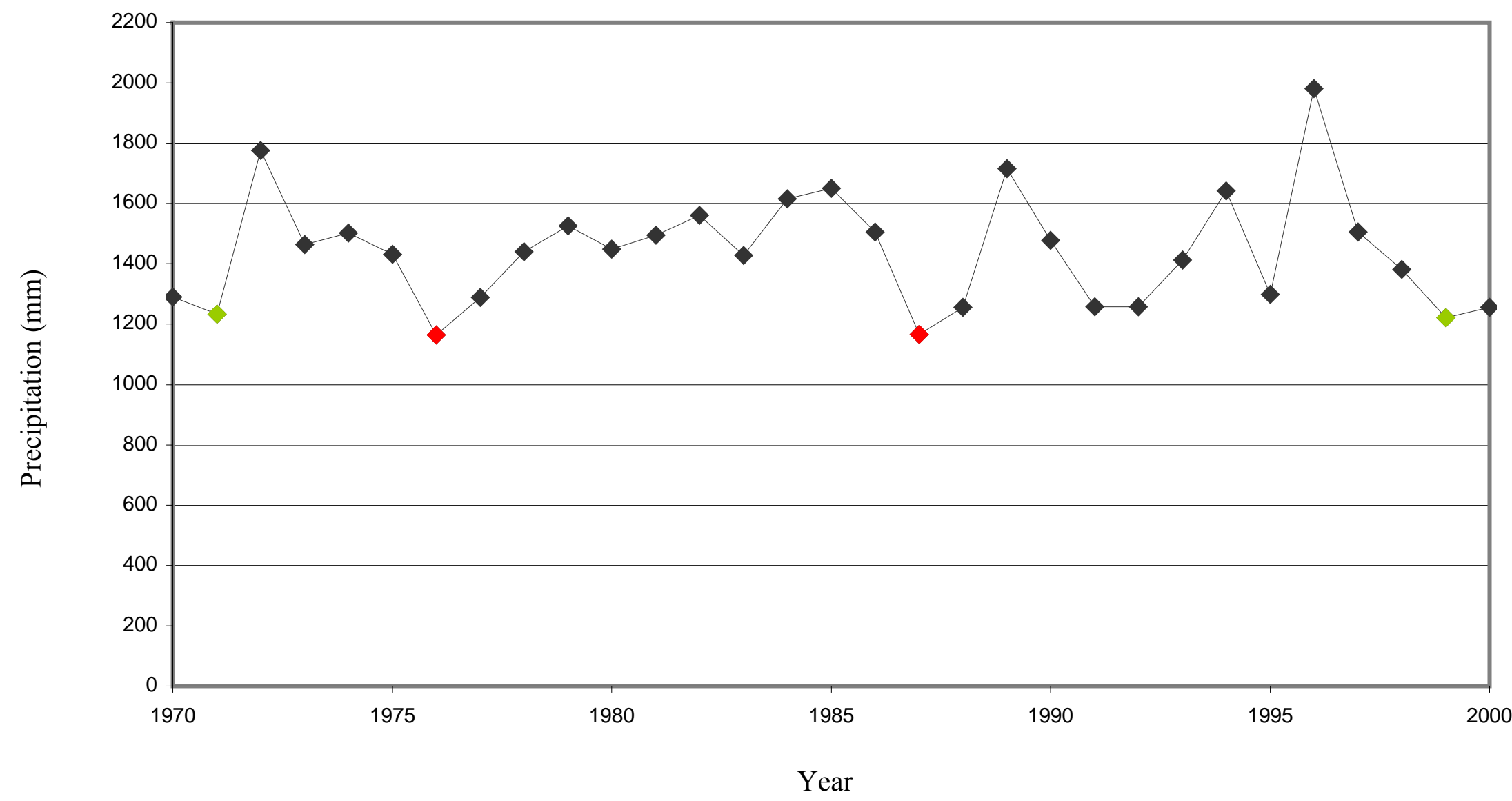

Figure 2. Fernow Experimental Forest total annual precipitation (1970-2000) with full-drought (red diamonds), partial-drought (green diamonds), and non-drought (gray diamonds) years plotted. 
Fernow Experimental Forest Growing Season Precipitation Data (1970-2000) WS7

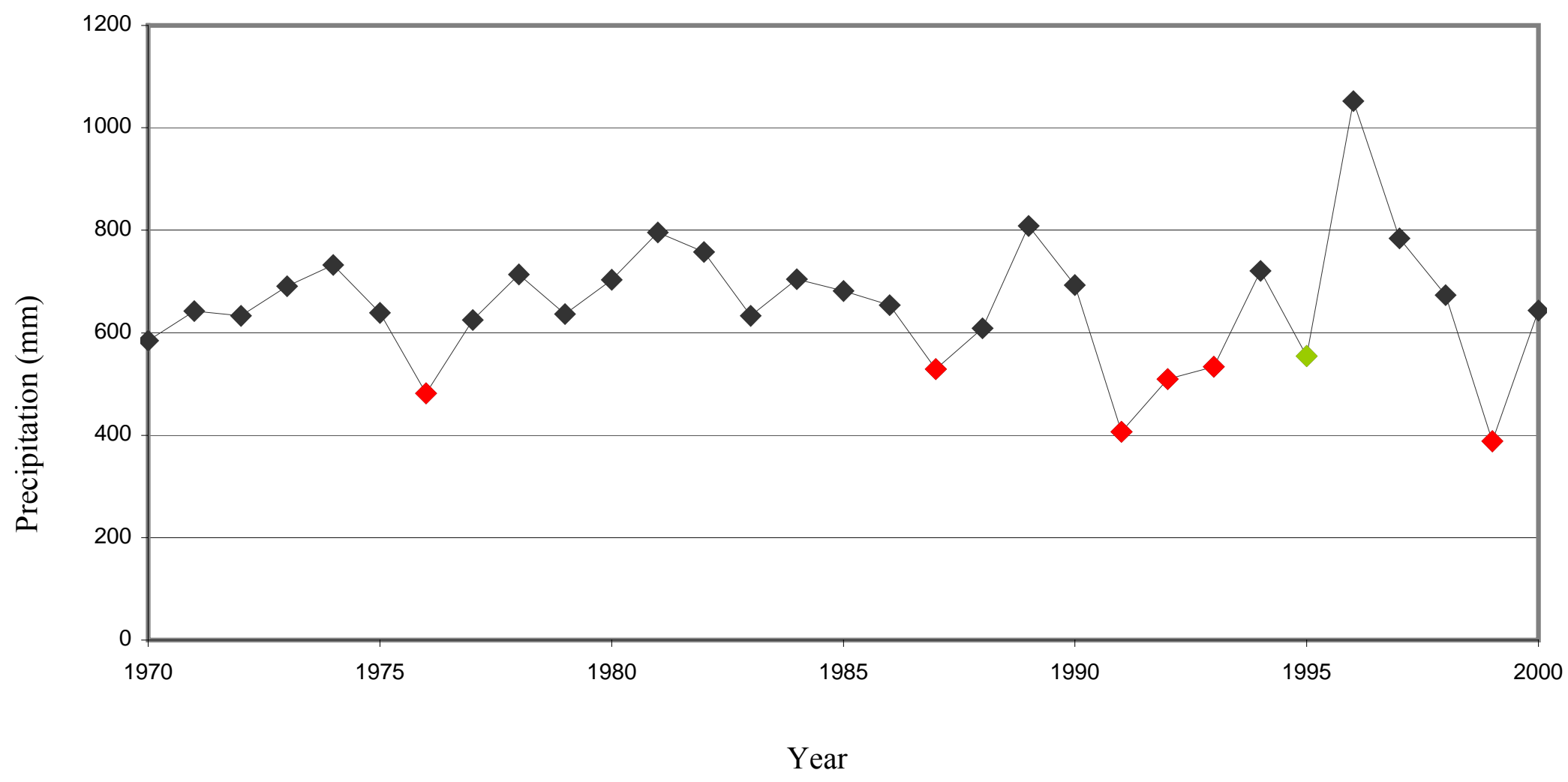

Figure 3. Fernow Experimental Forest growing season (May-September) precipitation (1970-2000) with full-drought (red diamonds), partial-drought (green diamonds), and non-drought (gray diamonds) years plotted. 
The Palmer Drought Severity Index (PDSI) is a widely used index that addresses two of the most elusive properties of droughts, their intensity and their duration (Alley, 1984) (Table 1). Palmer developed the PDSI as a means of measuring the severity of drought using a waterbalance model, enabling him to develop his index at the regional level. In doing so, the PDSI has several advantages: 1) it can be applied throughout the U.S. (with some modifications for snow and/or frozen ground) and 2) it considers both precipitation and temperature and their combined influences on evapotranspiration, soil moisture and runoff. On the other hand, the PDSI has several disadvantages: 1) simulation of runoff by a water balance model is quite unreliable and difficult to determine; 2) measurement of variables that go into the PDSI index are problematic, due to the difficulty and indirectness of measurement; and 3) not enough information is known about the relationships between the simulated variables and the actual physical conditions.

Table 1. Palmer classes for wet and dry periods (Palmer, 1965).

\begin{tabular}{|c|c|}
\hline $\begin{array}{c}\text { Palmer Drought Severity } \\
\text { Index Value }\end{array}$ & Class \\
\hline$\geq 4.00$ & Extremely wet \\
\hline 3.00 to 3.99 & Very wet \\
\hline 2.00 to 2.99 & Moderately wet \\
\hline 1.00 to 1.99 & Slightly wet \\
\hline 0.50 to 0.99 & Incipient wet spell \\
\hline 0.49 to -0.49 & Near normal \\
\hline-0.50 to -0.99 & Incipient drought \\
\hline-1.00 to -1.99 & Mild drought \\
\hline-2.00 to -2.99 & Moderate drought \\
\hline-3.00 to -3.99 & Severe drought \\
\hline$\leq-4.00$ & Extreme drought \\
\hline
\end{tabular}


1970-1995 PDSI Values (Cook et al ., 1999) Cell 135

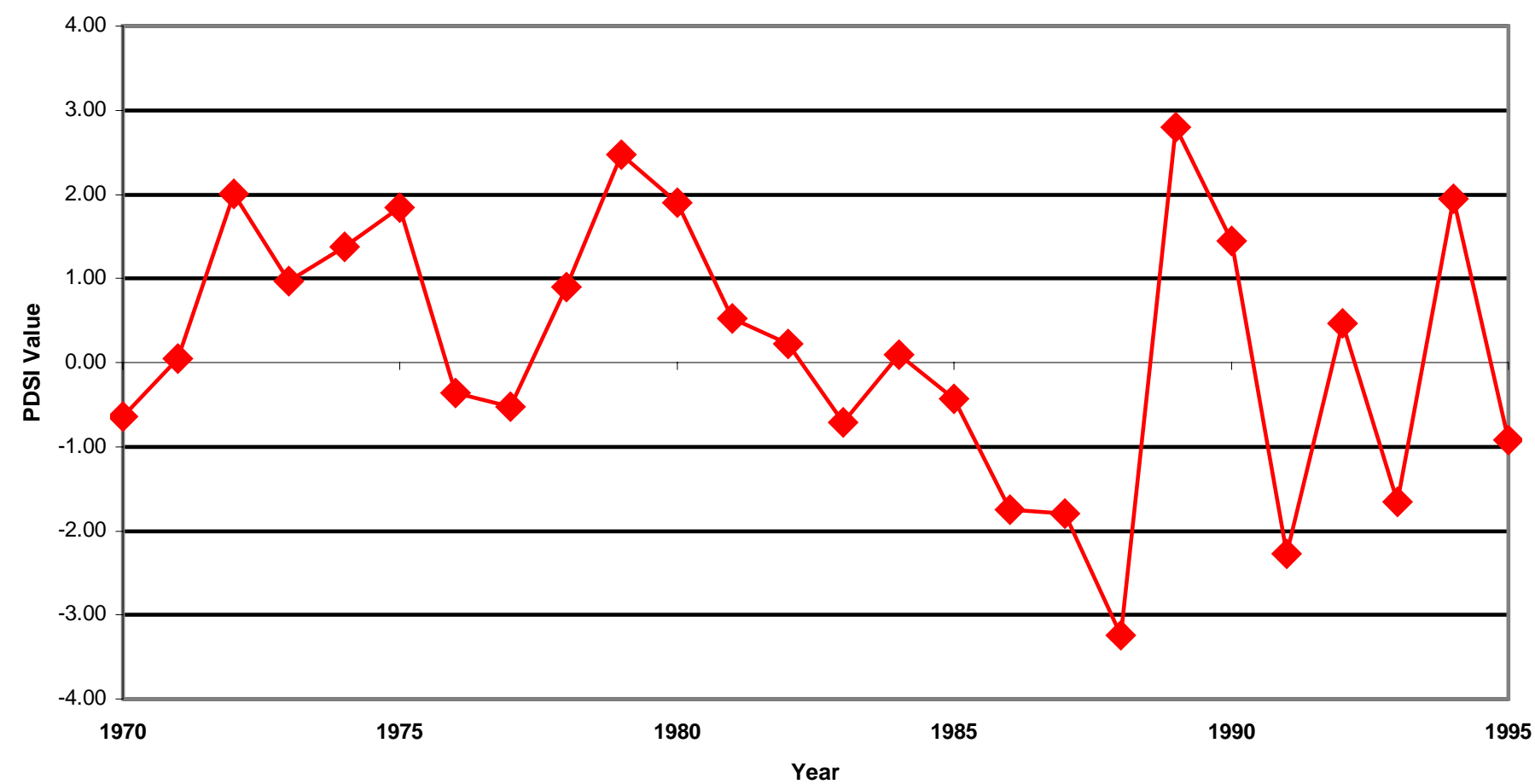

Figure 4. 1970-1995 PDSI values (grid point135) (Cook et al., 1999). 


\section{METHODS}

\section{$\underline{\text { Study Sites }}$}

The study was conducted in two watersheds (watershed 7 and watershed 13) in the

Fernow Experimental Forest, near Parsons, West Virginia (Table 2 and Figure 5). The Fernow

Experimental Forest is part of the Monongahela National Forest, and is located at $39^{\circ} 05^{\prime} \mathrm{N}, 79^{\circ}$

41'W (Adams et al., 1994).

Table 2. Physical and ecological characteristics of watershed 7 and watershed 13 (Adams et al., 1994).

\begin{tabular}{|c|c|c|}
\hline Attribute & Watershed 7 & Watershed 13 \\
\hline Size & $24.23 \mathrm{ha}$ & $14.23 \mathrm{ha}$ \\
\hline Elevation & $729-857 \mathrm{~m}$ & $715-808 \mathrm{~m}$ \\
\hline Aspect & Eastern & North-northeastern \\
\hline $\begin{array}{c}\text { Management } \\
\text { History }\end{array}$ & $\begin{array}{c}\text { Clear-cut in two phases (1963-1964 } \\
\text { and 1966-1967), left barren with } \\
\text { herbicides until 1969, then released }\end{array}$ & $\begin{array}{c}\text { No clear-cut or treatment since the } \\
\text { early part of the 20 }\end{array}$ \\
\hline Dominant trees & BELE, LITU, PRSE* & ACSA, FAGR, QURU \\
\hline Age of trees & $\leq 30$ years old; even aged stand & $\leq 90$ years old, uneven aged stand \\
\hline Simpson index & 0.166 & 0.292 \\
\hline Shannon index & 2.087 & 1.610 \\
\hline
\end{tabular}

*Tree species and abbreviations are located in Appendix A. 


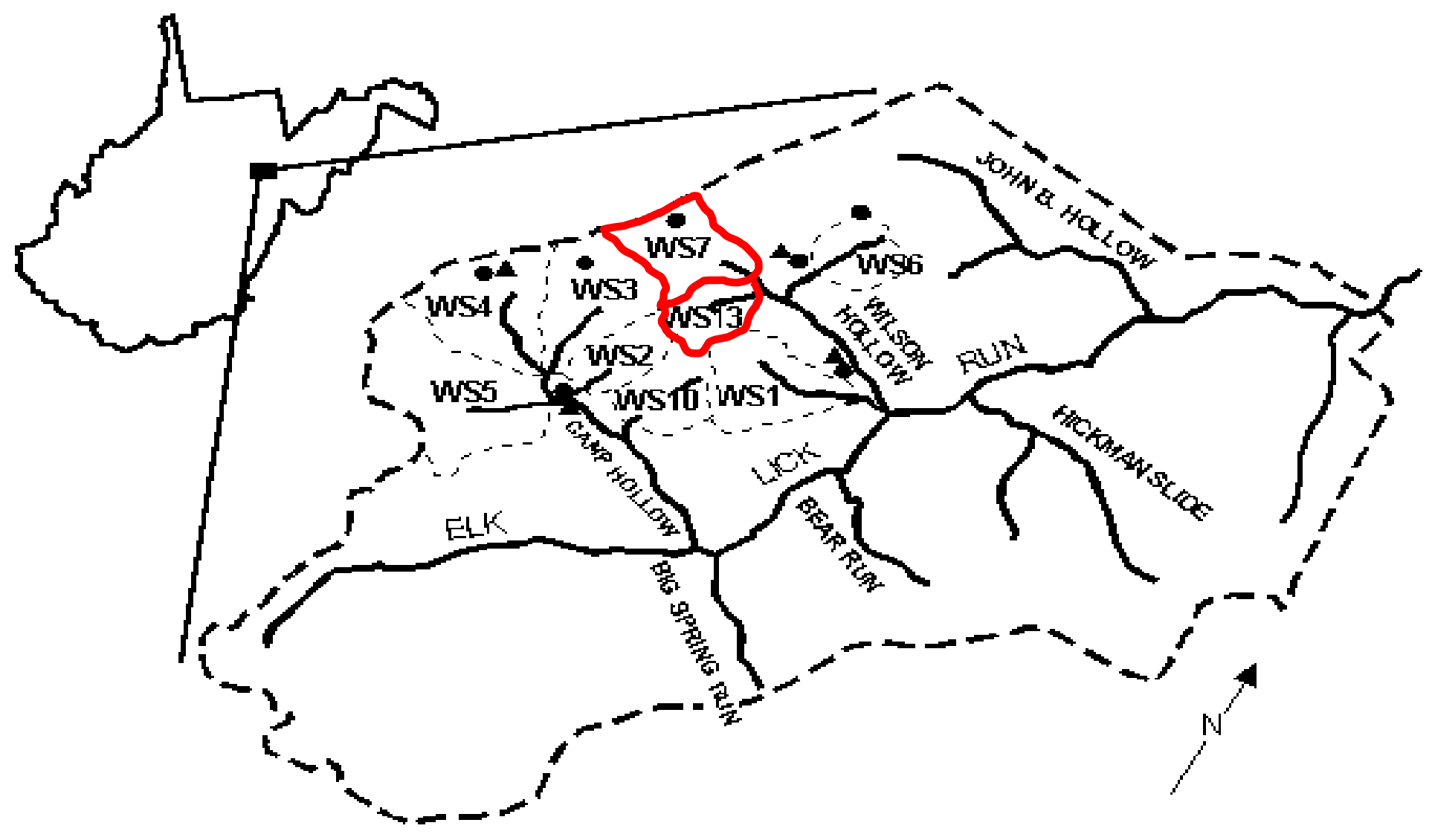

Figure 5. Locations of watershed 7 (WS 7) and watershed 13 (WS 13) in relation to one another and other watersheds at the Fernow Experimental Forest, Parsons, West Virginia (Adams et al., 1994). 
The climate of the two watersheds is moderate, with a mean annual precipitation of $1421 \mathrm{~mm}$ from 1957-2000 (Adams et al., 1994). In general, precipitation is distributed relatively evenly throughout the year, however, March through August have the greatest amount of precipitation. The average January temperature range is $-1^{\circ} \mathrm{C}$ to $5^{\circ} \mathrm{C}$, while the average July temperature is $25^{\circ} \mathrm{C}$. The soils in both watersheds are relatively thin $(<1 \mathrm{~m}$ in depth), acidic, sandy-loam Inceptisols of the Berks and Calvin series (Gilliam et al., 1995). The soil texture is on average roughly $67 \%$ sand, $11 \%$ clay, and $22 \%$ silt. Species richness and evenness were calculated using Simpson and Shannon indices to examine the possibly different species diversities and relative abundances of different species between the two watersheds (Schuler and Gillespie, 2000). These indices confirmed that the younger watershed is less species rich and even than the mature watershed (Table 2).

\section{Field Methods}

Six plots were established in each watershed, for a total of 12 study plots (Figure 6) (Table 3). Each plot was randomly chosen based on a $100 \mathrm{~m}$ x $100 \mathrm{~m}$ grid system, and located in the field using a GPS unit. A $10 \mathrm{~m}$ radius circle was then established at each selected grid intersection. All trees with a dbh (diameter at $1.37 \mathrm{~m}$ ) greater than $5 \mathrm{~cm}$ were included in a tree inventory. Tree species, dbh, tree height, crown height, and status (live or dead) for each tree were recorded (Appendix F). Two increment cores were taken from each tree at breast height $(1.37 \mathrm{~m})$. Each core was given an identification number and transported back to the laboratory for analysis. The increment boring was performed using standard tree-ring methods that do not harm the living tree (Stokes and Smiley, 1996). 


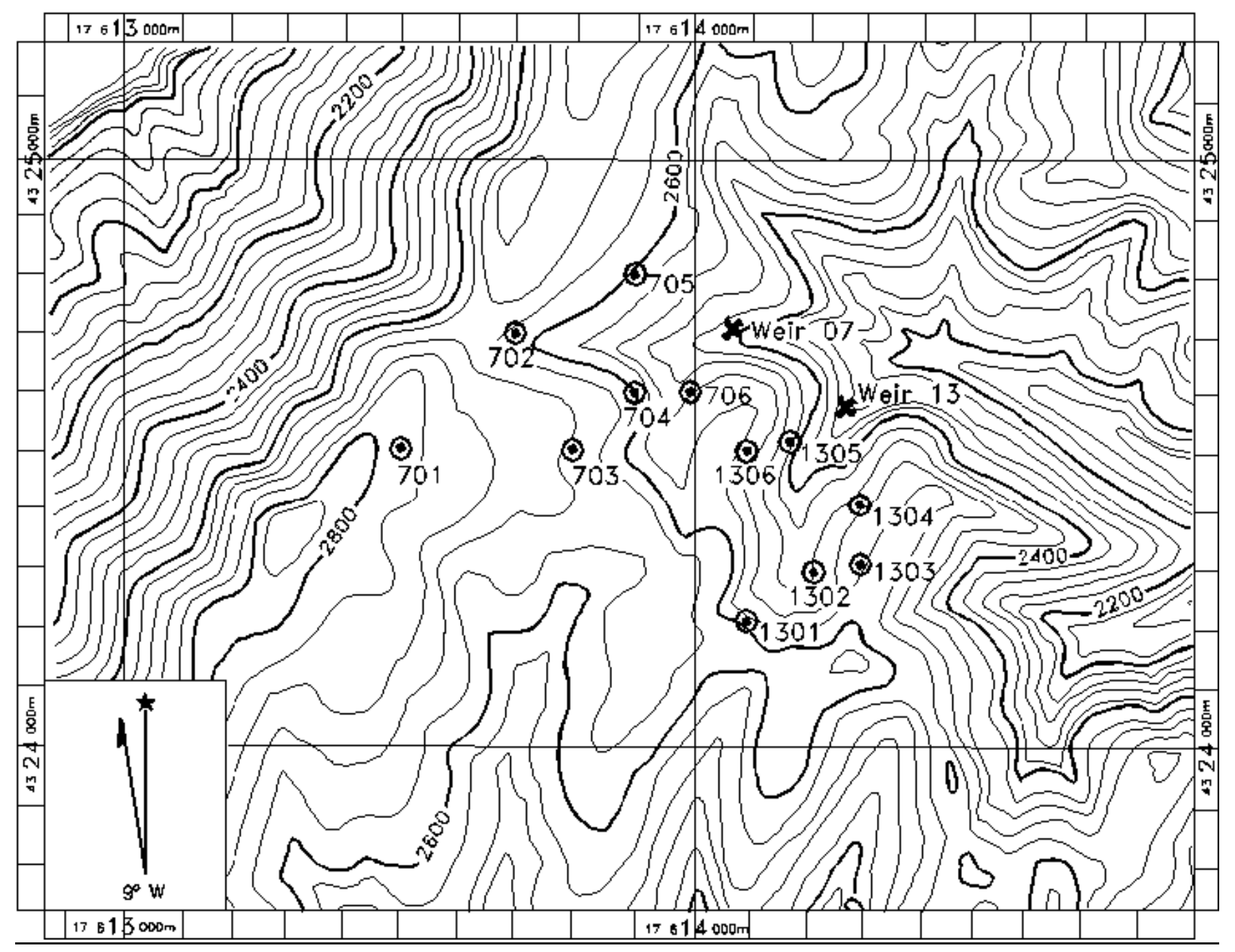

Figure 6. Location of the 12 study plots (circles) in watershed 7 and watershed 13 (distances expressed in UTM). 
Table 3. Aspect, slope, and elevation position for the 12 plots in watershed 7 and watershed 13.

\begin{tabular}{|l|c|c|c|}
\hline Plot & Aspect $\mathbf{(}^{\mathbf{}}$ ) & Slope (\%) & $\begin{array}{c}\text { Topographic } \\
\text { Position }\end{array}$ \\
\hline $\mathbf{1 3 0 1}$ & 29 & 50 & ridge-top \\
\hline $\mathbf{1 3 0 2}$ & 286 & 33 & mid-slope \\
\hline $\mathbf{1 3 0 3}$ & 350 & 18 & valley \\
\hline $\mathbf{1 3 0 4}$ & 327 & 18 & mid-slope \\
\hline $\mathbf{1 3 0 5}$ & 118 & 35 & mid-slope \\
\hline $\mathbf{1 3 0 6}$ & 319 & 20 & ridge-top \\
\hline $\mathbf{7 0 1}$ & 75 & 8 & ridge-top \\
\hline $\mathbf{7 0 2}$ & 51 & 10 & valley \\
\hline $\mathbf{7 0 3}$ & 99 & 13 & mid-slope \\
\hline $\mathbf{7 0 4}$ & 94 & 16 & mid-slope \\
\hline $\mathbf{7 0 5}$ & 166 & 18 & mid-slope \\
\hline $\mathbf{7 0 6}$ & 319 & 25 & valley \\
\hline
\end{tabular}

\section{$\underline{\text { Laboratory Preparation }}$}

Each increment core was prepared for measurement by mounting and then sanding, beginning with coarse-grit sandpaper (100-grit) and then using finer-grit sandpaper until 400-grit sandpaper was used to finish the smoothing process. Crossdating using skeleton plotting and marker years was attempted on the mature watershed's cores. With a maximum of 30 years of increment growth, the young watershed's cores were too short to be crossdated with confidence. Following measurement and subsequent crosschecking with COFECHA, a method of quality control on measured ring-widths, crossdating of the mature watershed's cores did not appear to be better than ring counting due to a lack of marker years. Approximately $10 \%$ of the increment cores were recounted to determine whether errors had been made in ring counting. It was concluded that the measurements and ring counts were correct, therefore, only "ring counting," is used to describe the corresponding year for each tree ring in both watersheds. 


\section{Biomass/Net Primary Productivity Calculations}

Tree species classification, dbh, bark coefficients, and ring measurements were used in combination with allometric equations (Brenneman et al. 1978), to calculate yearly biomass estimates for 1970-2002. Yearly diameters were calculated using:

$$
D_{t-1}=\frac{\left[D_{t}-\left(1-B_{s}\right) * D_{t}-2 * R_{t}\right]}{B_{s}}
$$

where $D$ is the dbh at a given time (t or $t-1), B_{s}$ is the bark coefficient for a given species, and $R_{t}$ is the ring width at time t. Yearly biomass values were calculated using species-specific equations (when applicable) or a general hardwood equation (Appendices B, E, F, and G).

From the individual aboveground tree biomass estimates, approximations of ANPP were calculated using:

$$
B_{t}-B_{t-1}=A
$$

where $\mathrm{B}_{t}$ is the biomass for year $\mathrm{t}, \mathrm{B}_{\mathrm{t}-1}$ is the biomass for the previous year, and $\mathrm{A}$ is the ANPP excluding litterfall. If a tree had more than one increment core, the annual ANPP of all cores was averaged after the calculations. Basal areas for each plot were calculated, using the following equation, to examine possible relationships to variability of plot ANPP:

$$
T B A=\pi r^{2}
$$

where TBA is the total basal area of a living tree and $r$ is the radius of the tree at breast height $(\mathrm{dbh} / 2)$.

\section{$\underline{\text { Litterfall }}$}

Litterfall data was collected to examine the possible yearly patterns associated with drought years, as well as to determine if Brenneman et al. (1978) was correct in using an acrossthe-board 5\% deduction for litterfall, using trees with ages ranging from 37 to 80 years, in his

allometric equations used here. Two litterfall collection containers (each $0.23 \mathrm{~m}^{2}$ ) were placed at 
each study plot to determine the amount of biomass released through litterfall by the standing trees throughout the year. Litterfall was collected every two weeks from September 6, 2002, to November 16, 2002, to ensure that little to no decomposition occurred (Clark et al., 2001). Collection containers that had been turned over were repositioned in order to collect litterfall. Plot 703 litterfall collection containers were stolen midway through litterfall collection.

Subsequently, the litterfall collection containers were replaced, and the time period (three weeks) of data was lost. After collection, litterfall was dried and then separated by species. Litterfall that could not be identified to species, as well as twigs and bare stems, were placed into an unknown/misc. category. After drying and separation, the litterfall was weighed (Table 5b). The plot total was then calculated by summing the species totals from both baskets and then extrapolating to the total plot area (Appendix D).

Litterfall weights were also obtained for 1991-2002 by the Fernow staff (Table 5a)

(Fernow Experimental Forest, 2003) from five existing $1 \mathrm{~m}^{2}$ litterfall traps located near my newly established plots. This close proximity allowed comparisons between their measurements and my measurements (Adams et al., 1995). Fernow litterfall data was collected, dried, and weighed multiple times throughout the fall season. Only the total weight for each collection basket was recorded, thus there was no separation by species.

\section{Precipitation Data}

Total annual and growing-season precipitation from the Fernow Experimental Forest was summarized from daily precipitation data for 1970-2000 (Adams et al., 1994; Fernow Experimental Forest, 2003). Years or growing-seasons with a 15\% or more decrease in precipitation from the 30-year growing-season average were considered drought years (Figures 2 
and 3). Additional drought information included annual instrumental Palmer Drought Severity Index data for grid point 135, located at 3900’ N, 80³0’W (Figure 4) (Cook et al., 1999).

\section{$\underline{\text { Statistical Analysis }}$}

The ANPP estimates were compared with annual and growing-season (May - September) precipitation data using a variety of statistical techniques. To determine whether there is a significant difference in ANPP of young versus mature watersheds, I used an ANOVA (Hypothesis I). An analysis of covariance (ANCOVA) was used to determine whether there is a difference in drought's effect on the aboveground productivity of young versus mature watersheds (Hypothesis II). An analysis of covariance (ANCOVA) was used to identify whether there are species-specific effects of drought on productivity (Hypothesis III). For both hypotheses II and III, two sets of ANCOVAs were performed to determine if there is a possible lag time between amount of precipitation and ANPP (sensu Orwig and Abrams 1997). 


\section{RESULTS}

\section{Tree Survey}

Of the 404 trees surveyed, 110 living trees were sampled in the mature watershed and 206 living trees were sampled in the young watershed, for a total of 316 living trees. There were 21 different species present, however most of the living trees $(83.5 \%)$ consisted of only six species (Table 4 and Appendix C). Summary of the ring width data between the two watersheds demonstrates that trees in the young watershed have larger average ring widths than trees in the mature watershed (Table 5).

Table 4. Frequency of tree species sampled in watershed 7 and watershed 13.

\begin{tabular}{|c|c|c|c|c|}
\hline Species & Total \# & WS 7 \# (\%) & WS 13 \# (\%) & Total \% \\
\hline ACSA & 73 & $23(11.2)$ & $50(45.5)$ & 23.1 \\
\hline BELE & 61 & $61(29.6)$ & $0(0.0)$ & 19.3 \\
\hline FAGR & 39 & $11(5.3)$ & $28(25.5)$ & 12.3 \\
\hline LITU & 39 & $38(18.4)$ & $1(0.9)$ & 12.3 \\
\hline PRSE & 30 & $30(14.6)$ & $0(0.0)$ & 9.5 \\
\hline QURU & 22 & $8(3.9)$ & $14(12.7)$ & 7.0 \\
\hline Other & 52 & $35(17.0)$ & $17(15.5)$ & 16.5 \\
\hline Total & 316 & 206 & 110 & 100.0 \\
\hline
\end{tabular}

Table 5. Summary of ring width measurements for watershed 7 and watershed 13 expressed in $\mathrm{cm}$.

\begin{tabular}{|c|c|c|c|c|}
\hline Species & $\begin{array}{c}\text { WS 7 } \\
\text { (Average) }\end{array}$ & $\begin{array}{c}\text { WS 7 } \\
\text { (Standard } \\
\text { deviation) }\end{array}$ & $\begin{array}{c}\text { WS 13 } \\
\text { (Average) }\end{array}$ & $\begin{array}{c}\text { WS 13 } \\
\text { (Standard } \\
\text { deviation) }\end{array}$ \\
\hline ACSA & 0.149 & 0.069 & 0.110 & 0.061 \\
\hline BELE & 0.230 & 0.076 & --------- & --------- \\
\hline FAGR & 0.224 & 0.150 & 0.088 & 0.050 \\
\hline LITU & 0.394 & 0.149 & 0.246 & --------- \\
\hline Whole watershed & 0.263 & 0.132 & 0.130 & 0.085 \\
\hline
\end{tabular}




\section{$\underline{\text { Litterfall }}$}

Average weight of the litterfall collected in the two watersheds reflects little difference with regards to the maturity of the trees in watershed 13 and the high tree density found in watershed 7. If the assumption was made that plot 703, which experienced a loss of data, litterfall was similar to the overall numbers found throughout the remaining plots, then the total watershed weights among the two watersheds would be relatively similar. Thus, leaf production and subsequent litterfall appear to be similar with little to no substantial difference due to stand age. Furthermore, litterfall weights taken for 2002 are greater than the 12-year Fernow average, however, similar weights can be observed several times throughout the 12-year collection history provided by the Fernow staff (Table 6 and Appendix D). Litterfall production appears to decrease substantially, by up to $25 \%$, during drought episodes, such as 1997 . On the other hand, a substantial increase in precipitation does not necessarily mean that more litterfall will be produced; such is the case in 1994 and 1996. Litterfall weights were compared to the 2002 biomass calculations for each watershed. This work demonstrated that Brenneman et al. (1978) was incorrect in using an across-the-board 5\% deduction for litterfall in his allometric equations. A more reliable value for litterfall relative to total biomass would be $2-5 \%$ for mature forests and $12-15 \%$ for young forests. However, my results do not fully go against Brenneman et al.'s (1978) work. The ages of the trees examined ranged from 37 to 80 years, with the overall average of 63 years (Brenneman et al., 1978). Thus, Brenneman et al. (1978) only examined mature trees, where a 5\% deduction was a reliable value to use. 
Table 6. Summary of litterfall weight for the Fernow from 1991-2002 for selected collection baskets in watershed 7, expressed in $\mathrm{kg} / \mathrm{ha} / \mathrm{year}$ (a), and for 2002 in newly established collection baskets in watershed 7 and watershed 13, expressed in $\mathrm{kg} / \mathrm{ha} / \mathrm{year}(\mathbf{b})$.

(a)

\begin{tabular}{|c|c|c|c|c|c|c|c|c|c|c|c|c|c|}
\hline $\begin{array}{c}\text { Collection } \\
\#\end{array}$ & $\mathbf{1 9 9 1}$ & $\mathbf{1 9 9 2}$ & $\mathbf{1 9 9 3}$ & $\mathbf{1 9 9 4}$ & $\mathbf{1 9 9 5}$ & $\mathbf{1 9 9 6}$ & $\mathbf{1 9 9 7}$ & $\mathbf{1 9 9 8}$ & $\mathbf{1 9 9 9}$ & $\mathbf{2 0 0 0}$ & $\mathbf{2 0 0 1}$ & $\mathbf{2 0 0 2}$ & $\begin{array}{c}\text { 12-Year } \\
\text { Average }\end{array}$ \\
\hline $\mathbf{5 6}$ & 2935 & 3180 & 2828 & 2778 & 2763 & 2511 & 1878 & 2326 & 2337 & 3163 & 3092 & 2785 & 2715 \\
\hline $\mathbf{6 0}$ & 3081 & 2789 & 2956 & 3300 & 3202 & 2750 & 1949 & 2554 & 2968 & 3452 & 3414 & 3022 & 2953 \\
\hline $\mathbf{6 9}$ & 2358 & 2685 & 1817 & 1927 & 2095 & 2081 & 1755 & 1792 & 1838 & 1754 & 2697 & 2178 & 2081 \\
\hline $\mathbf{7 2}$ & 2321 & 2204 & 2192 & 2014 & 2268 & 2488 & 1762 & 1775 & 2209 & 1612 & 3369 & 2079 & 2191 \\
\hline $\mathbf{7 4}$ & 2323 & 2251 & 2500 & 2149 & 2361 & 2064 & 1548 & 1904 & 1728 & 1649 & 2281 & 2068 & 2069 \\
\hline
\end{tabular}

(b)

\begin{tabular}{|c|c|}
\hline Plot & 2002 \\
\hline $\mathbf{7 0 1}$ & 3288 \\
\hline $\mathbf{7 0 2}$ & 3688 \\
\hline $\mathbf{7 0 3}$ & $1298^{*}$ \\
\hline $\mathbf{7 0 4}$ & 3284 \\
\hline $\mathbf{7 0 5}$ & 1384 \\
\hline $\mathbf{7 0 6}$ & 2964 \\
\hline $\mathbf{1 3 0 1}$ & 3760 \\
\hline $\mathbf{1 3 0 2}$ & 1644 \\
\hline $\mathbf{1 3 0 3}$ & 3520 \\
\hline $\mathbf{1 3 0 4}$ & 3398 \\
\hline $\mathbf{1 3 0 5}$ & 3936 \\
\hline $\mathbf{1 3 0 6}$ & 3130 \\
\hline WS 7 & $2922^{* *}$ \\
\hline WS 13 & 3231 \\
\hline
\end{tabular}

*Three weeks of litterfall data during the fall was lost due to stolen collection baskets.

**Average was calculated excluding plot 703 .

\section{Productivity}

There were a total of 738 increment cores taken from live and dead trees in both watersheds. 41 increment cores were rotten or unreadable, thus 697 increment cores were 
measured. However all dead tree increment cores were removed from ANPP calculations due to crossdating problems.

During the 1971-2001 time period, the mature watershed's plots had greater mean ANPP than the young watershed's plots due to the larger size (height and diameter) of the trees in the mature watershed and lower ANPP values of the young watershed during the beginning years of its re-vegetation (Table 7). However, towards the later part of the 30-year period examined, the ANPP values of the young watershed were greater than observed values in the mature watershed, thus the mean ANPP, appears to be similar in value. The mature watershed's plots also have higher mean biomass compared to the young watershed's plots. However, great variability was also found among plots in the same watershed, especially the young watershed, which had more ANPP outliers (Figures 7 and 8).

Table 7. Mean ANPP excluding litterfall (1971-2001) expressed in $\mathrm{kg} / \mathrm{ha} / \mathrm{year}$, and biomass (2002) for plots in watershed 7 and watershed 13 expressed in $\mathrm{kg} / \mathrm{ha}$.

\begin{tabular}{|c|c|c|c|c|}
\hline & $\begin{array}{c}\text { Mean } \\
\text { ANPP } \\
\text { (kg/ha/yr) }\end{array}$ & $\begin{array}{c}\text { Standard deviation } \\
\text { ANPP (kg/ha/yr) }\end{array}$ & $\begin{array}{c}\text { Mean } \\
\text { biomass } \\
\text { (kg/ha) }\end{array}$ & $\begin{array}{c}\text { Standard deviation } \\
\text { biomass (kg/ha) }\end{array}$ \\
\hline WS 7 & 5344.7 & 1681.6 & 166163.2 & 53194.3 \\
\hline WS 13 & 5655.1 & 2546.9 & 287454.6 & 149202.3 \\
\hline
\end{tabular}




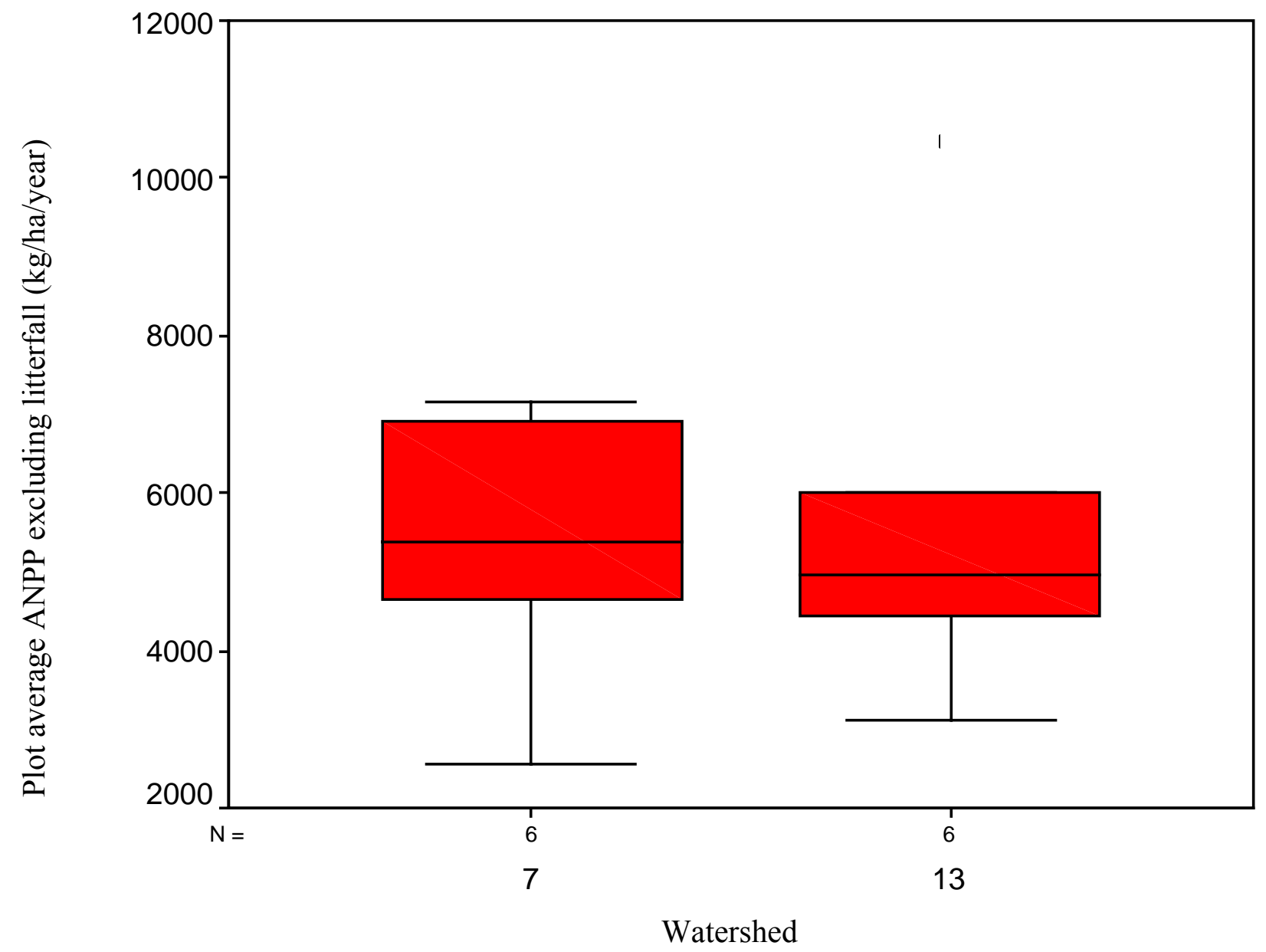

Figure 7. Box plots of plot average ANPP excluding litterfall (kg/ha/year) for watershed 7 and watershed 13 for 1971-2001, with the middle line showing the median. 


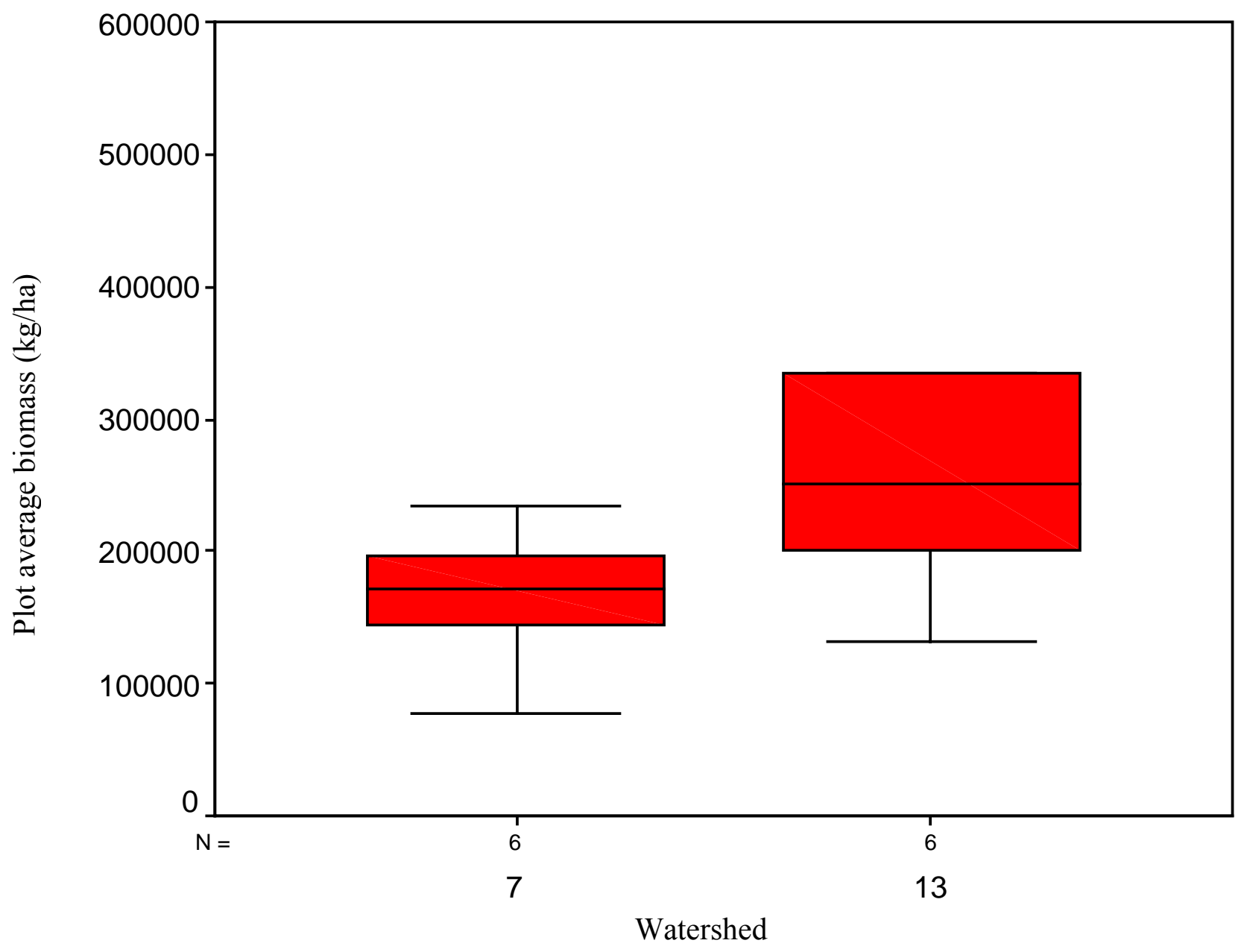

Figure 8. Box plots of plot average biomass ( $\mathrm{kg} / \mathrm{ha}$ ) for watershed 7 and watershed 13 for 2002, with the middle line showing the median. 
Substantial differences in the pattern of aboveground ANPP (kg/plot) for 1971-2001 in the two watersheds were found. The young watershed demonstrated increasing ANPP of a revegetating area as the number of trees and size of existing trees increased over time (Figure 9). In contrast, the mature watershed demonstrated a "leveled off" pattern of aboveground ANPP typical of a forested area that has reached a later stage of succession (Figure 10).

There was a substantial drop of ANPP in 1987, 1999 (both watersheds), and 1976 (watershed 13). These drops in ANPP coincided with corresponding drops in total annual precipitation and growing season precipitation (Figures 9 and 10). However, changes in ANPP only occurred with considerable decreases in precipitation, otherwise, precipitation (total annual precipitation, growing season precipitation, and PDSI) did not correlate well with ANPP. For the 1970-1995 time period, plots 701, 703 and 706 were only weakly negatively correlated with PDSI data $(\mathrm{R}=-0.381, \mathrm{P}=0.066 ; \mathrm{R}=-0.45, \mathrm{P}=0.099 ;$ and $\mathrm{R}=-0.370, \mathrm{P}=0.069$, respectively $)$. Furthermore, no plots were significantly correlated with total annual precipitation and only plot 1305 was significantly, but negatively, correlated with growing season precipitation $(R=-0.373$, $\mathrm{P}=0.043)($ Table $8 \mathrm{a})$. While the general pattern of correlation was weak, the correlation between ANPP and precipitation did become stronger when examining the longer time period (1957-2000) available with data from the mature watershed. For the mature watershed, correlations were most significant when examining the relationship between ANPP and growing season precipitation. Plots 1302, 1304, and 1306 were all found to be correlated with growing season precipitation $(\mathrm{R}=0.288, \mathrm{P}=0.058 ; \mathrm{R}=0.309, \mathrm{P}=0.041$; and $\mathrm{R}=0.308, \mathrm{P}=0.042$ respectively), while plot 1306 was also found to be weakly correlated with annual total precipitation $(\mathrm{R}=0.253, \mathrm{P}=0.098)($ Table $8 \mathrm{~b})$. 
WS7

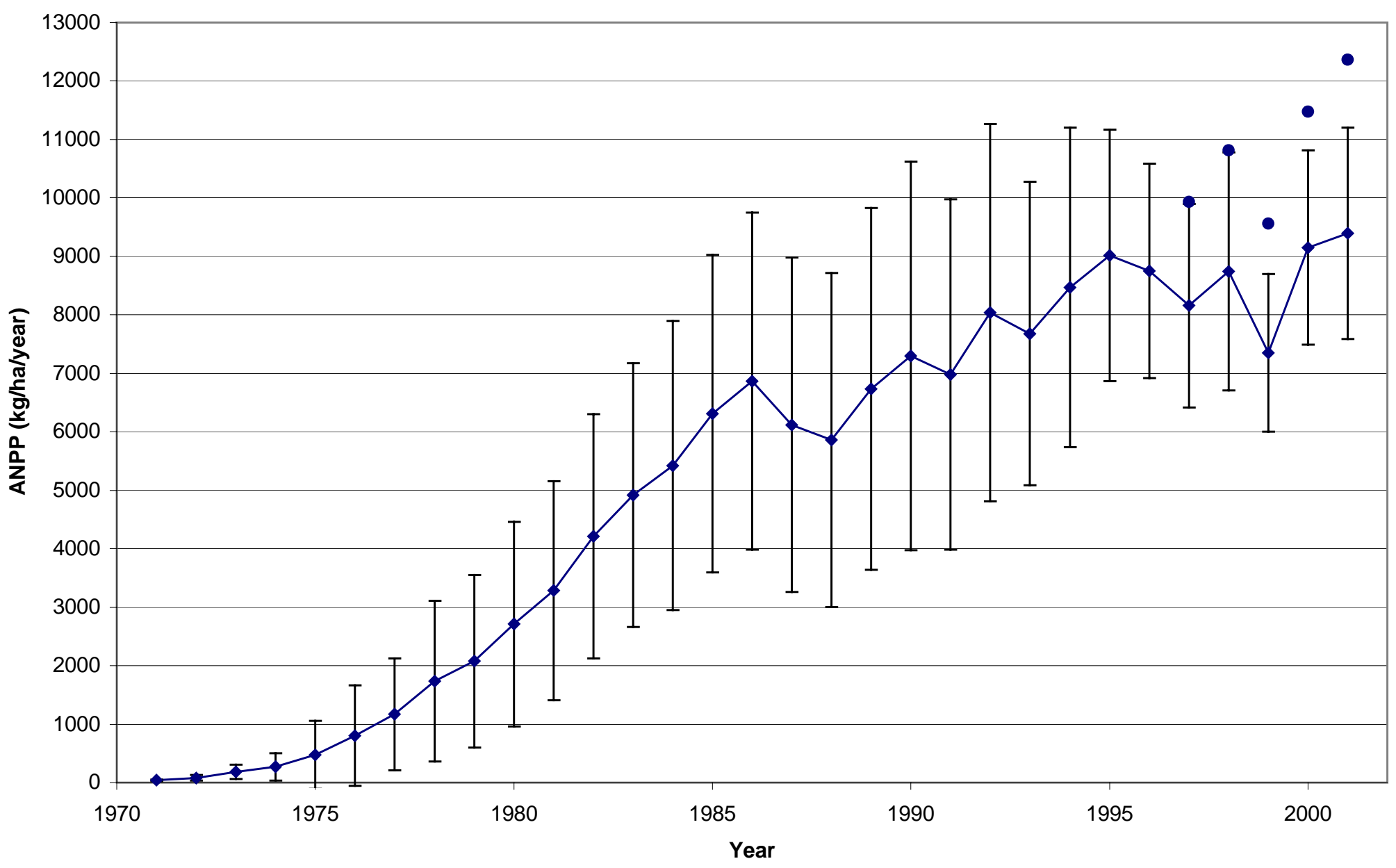

Figure 9. ANPP excluding litterfall (kg/ha/year) for watershed 7 (1971-2001) with diamonds showing estimated values, standard deviations bars; circles showing the estimated values including litterfall collected by the Fernow staff (WS 7). 
WS13

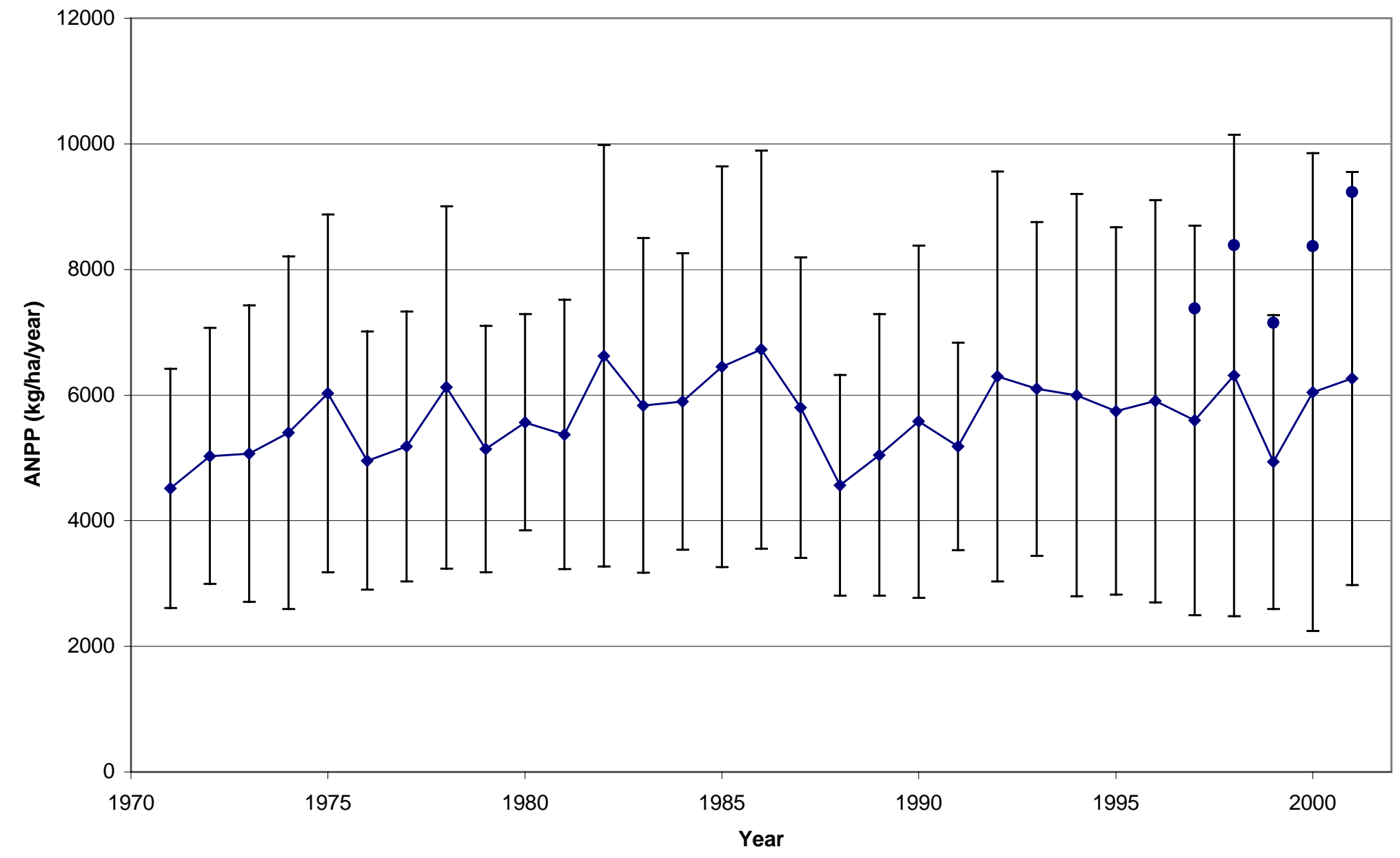

Figure 10. ANPP excluding litterfall (kg/ha/year) for watershed 13 (1971-2001) with diamonds showing estimated values, standard deviations bars; circles showing the estimated values including litterfall collected by the Fernow staff (WS 7). 
Table 8. Pearson correlation coefficients ( $r$ ) for the mean annual ANPP for each plot and total annual precipitation, growing season precipitation, and PDSI values for the 1971-1995 time period (a), and for the 1957-2000 time period (b).

(a)

\begin{tabular}{|c|c|c|c|}
\hline Plot & $\begin{array}{c}\text { Correlation to Annual } \\
\text { Precipitation }\end{array}$ & $\begin{array}{c}\text { Correlation to Growing Season } \\
\text { Precipitation }\end{array}$ & $\begin{array}{c}\text { Correlation to PDSI } \\
\text { values }\end{array}$ \\
\hline $\mathbf{7 0 1}$ & -0.050 & 0.040 & $-0.381^{* *}$ \\
\hline $\mathbf{7 0 2}$ & -0.012 & 0.029 & -0.239 \\
\hline $\mathbf{7 0 3}$ & 0.061 & 0.049 & $-0.345^{* *}$ \\
\hline $\mathbf{7 0 4}$ & 0.063 & 0.023 & -0.327 \\
\hline $\mathbf{7 0 5}$ & 0.079 & -0.016 & -0.357 \\
\hline $\mathbf{7 0 6}$ & 0.041 & -0.045 & $-0.370^{* *}$ \\
\hline $\mathbf{1 3 0 1}$ & 0.166 & 0.155 & -0.217 \\
\hline $\mathbf{1 3 0 2}$ & 0.159 & 0.109 & 0.047 \\
\hline $\mathbf{1 3 0 3}$ & 0.021 & -0.041 & -0.181 \\
\hline $\mathbf{1 3 0 4}$ & 0.271 & 0.294 & 0.090 \\
\hline $\mathbf{1 3 0 5}$ & -0.155 & $-0.373^{*}$ & -0.199 \\
\hline $\mathbf{1 3 0 6}$ & 0.206 & 0.249 & 0.041 \\
\hline
\end{tabular}

(b)

\begin{tabular}{|c|c|c|}
\hline Plot & $\begin{array}{c}\text { Correlation to Annual } \\
\text { Precipitation }\end{array}$ & $\begin{array}{c}\text { Correlation to Growing Season } \\
\text { Precipitation }\end{array}$ \\
\hline $\mathbf{1 3 0 1}$ & 0.137 & 0.195 \\
\hline $\mathbf{1 3 0 2}$ & 0.231 & $0.288^{* *}$ \\
\hline $\mathbf{1 3 0 3}$ & 0.122 & 0.157 \\
\hline $\mathbf{1 3 0 4}$ & 0.240 & $0.309^{*}$ \\
\hline $\mathbf{1 3 0 5}$ & 0.009 & 0.027 \\
\hline $\mathbf{1 3 0 6}$ & $0.253^{* *}$ & $0.308^{*}$ \\
\hline
\end{tabular}

* Significant at the $\alpha=0.05$.

** Significant at the $\alpha=0.10$.

Closer examination of each watershed, on the individual plot level, demonstrated that the declines of ANPP during drought events are also found to occur over the time period of the study. Each plot, with the exception of plot 1305, showed noteworthy decreases during the drought year of 1999, however, this appearance of a relation is not consistent with the correlation results. This is also apparent, but not as noteworthy, in the drought year of 1987. 
In watershed 7, each plot, with the exception of plot 702, has similar growth curves. In the case of plot 702, located in a topographic depression, growth was slow until 1990 after which trees grew at a greater rate (Figure 11). Two anomalies can be found in watershed 13. Plot 1305 acts counter to the norm throughout the entire study period when drought years occur (Figure 12). The most probable reason for this anomaly is the aspect of 1305 , which is $118^{\circ}$, or roughly ESE, which would effect the microclimate of the plot. Plot 1306, on the other hand, follows the norm, however at a much higher level of ANPP. After calculation of basal area, it is most likely that the existence of several extremely large individual trees gives plot 1306 a higher basal area, as well as a higher rate of ANPP (Figures 11 and 12) (Table 9). 
WS7

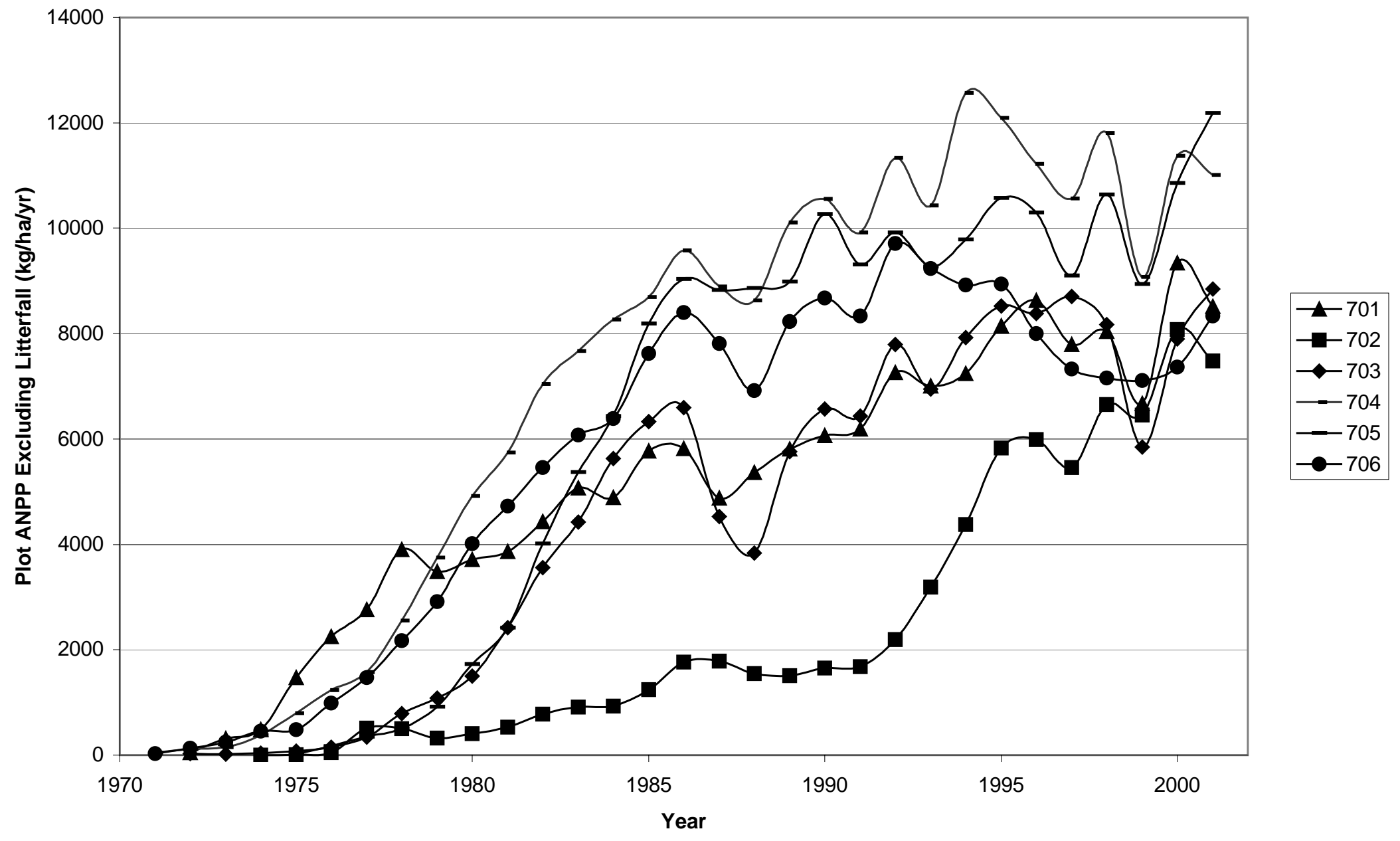

Figure 11. ANPP excluding litterfall (kg/ha/year) for watershed 7 plots for 1971-2001. 


\section{WS13}

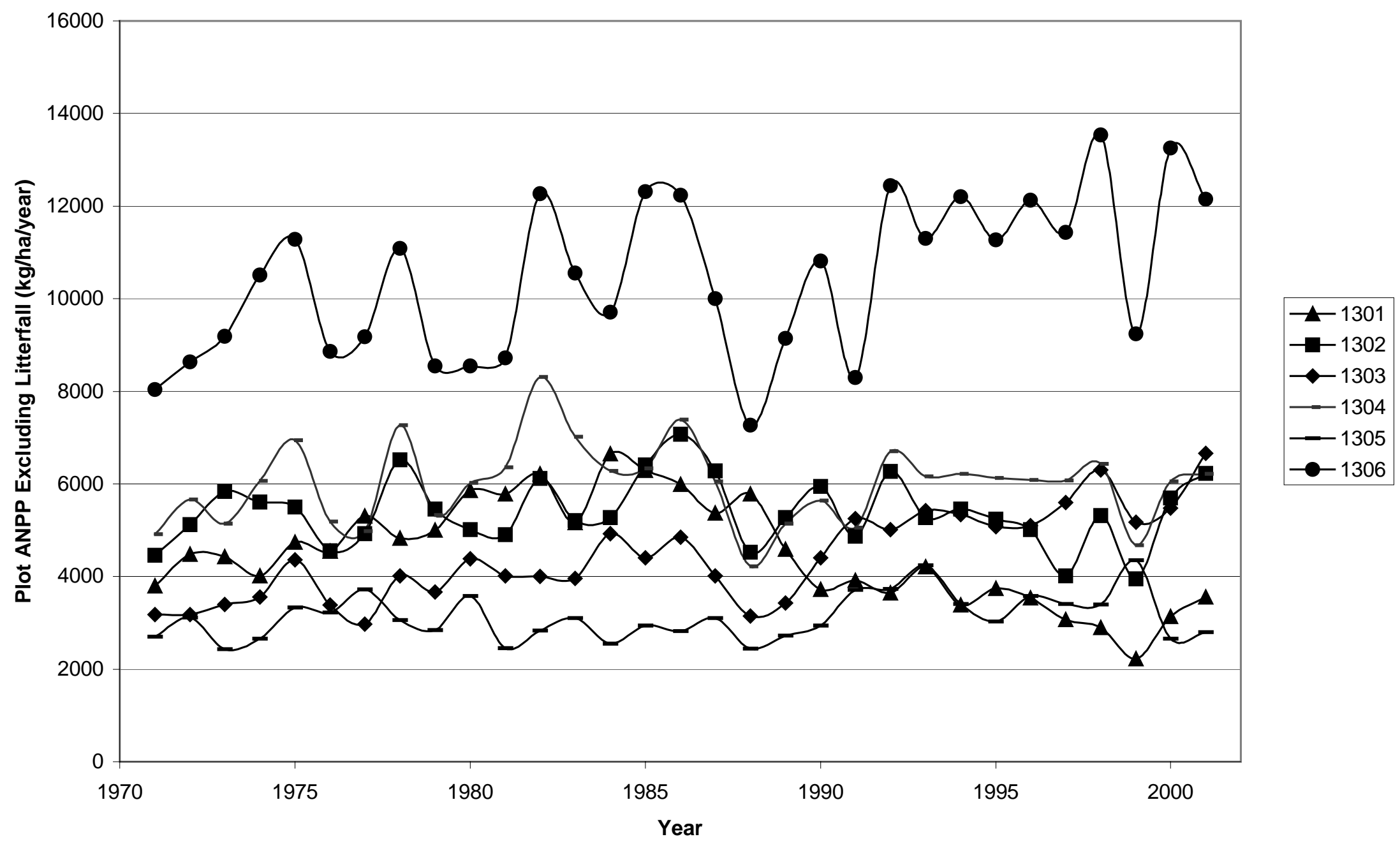

Figure 12. ANPP excluding litterfall (kg/ha/year) for watershed 13 plots for 1971-2001. 


\section{$\underline{\text { Basal Area }}$}

The basal area calculations do account for the higher plot ANPP found at 1306 as opposed to 1301, which lie at similar elevations (Table 9). The average basal area also explains the higher plot ANPP found in the mature watershed, as opposed to the young watershed, which is further evidence that in these watersheds tree size plays a greater role in productivity than tree density.

Table 9. Basal area for each plot and watershed average $\left(\mathrm{cm}^{2} / \mathrm{ha}\right)$.

\begin{tabular}{|c|c|c|}
\hline Plot \# & WS 7 & WS 13 \\
\hline $\mathbf{1}$ & 280,983 & 213,686 \\
\hline $\mathbf{2}$ & 125,661 & 345,688 \\
\hline $\mathbf{3}$ & 257,009 & 281,930 \\
\hline $\mathbf{4}$ & 364,021 & 399,112 \\
\hline $\mathbf{5}$ & 347,228 & 174,399 \\
\hline $\mathbf{6}$ & 315,334 & 584,379 \\
\hline Watershed Average & 281,706 & 333,199 \\
\hline
\end{tabular}

\section{Hypothesis I (ANPP and WS age)}

An ANOVA was run on the mean annual ANPP for each plot per year to determine if young and mature watersheds have a significantly different rate of ANPP. Over the first 15 years of the study, the average annual ANPP rate of the mature watershed was higher than the young watershed, while in the second 15 years of the study, the young watershed had a higher rate of growth than the mature watershed. Thus, while there is a substantial difference in rate over the entire period in the younger watershed, the overall average was relatively similar to the older watershed $(\mathrm{F}=0.02, \mathrm{P}=0.889)($ Table 10$)$.

Hypothesis II (ANPP, WS age, and drought)

An ANCOVA was performed on the mean annual ANPP with regards to drought classifications (annual totals and growing season totals) to determine if drought events affect the 
ANPP in different aged watersheds differently; thus watershed was the covariate in the model in order to address the significant difference between the two watersheds found in hypothesis I. Two ANCOVAs were run. One examined annual ANPP relative to same year classification, while the second ANCOVA examined annual ANPP relative to the previous year classification.

\section{$\underline{\text { Same Year Classification }}$}

Over all years (1971-2001), the effect of same year drought classification is associated with a lower value of ANPP $(\mathrm{F}=2.72, \mathrm{P}=0.100)$. However, the same year growing season drought classification does not appear to be associated with the rate of ANPP $(F=1.75$, $\mathrm{P}=0.186)($ Table 10).

\section{$\underline{\text { Previous Year Classification }}$}

Over all years (1971-2001), the previous year's (1970-2000) annual drought classification does not appear to play a significant role in the following year's ANPP $(\mathrm{F}=0.30, \mathrm{P}=0.585)$. However, the previous year's growing season classification appears to be associated with a much greater role than the same year growing season classification $(F=7.36, P=0.007)($ Table 10$)$.

Hypothesis III (ANPP, tree species, and drought)

An ANCOVA was used to identify whether there are species-specific effects of drought with regards to the four most common species (ACSA, BELE, FAGR, and LITU (tree species and abbreviations are located in Appendix A)). This statistical test determined if drought events would affect the ANPP of different temperate tree species differently. Two ANCOVAs were run. One examined annual ANPP relative to same year drought classification, while the second ANCOVA examined annual ANPP relative to the previous year drought classification. 


\section{$\underline{\text { Same Year Classification }}$}

Over all years (1971-2001), species does not affect the relationship between same year drought classification and ANPP $(\mathrm{F}=0.93, \mathrm{P}=0.334)$. Furthermore, species does not affect whether the same year growing season drought classification has an effect on the rate of ANPP $(\mathrm{F}=0.02, \mathrm{P}=0.895)($ Table 10$)$.

\section{$\underline{\text { Previous Year Classification }}$}

Over all years (1971-2001), the effects of species may play a more notable role with regards to the previous year's (1970-2000) annual drought classification $(\mathrm{F}=2.70, \mathrm{P}=0.101)$, however this role is not significant at the $\alpha=0.05$ level. On the other hand, the previous year's growing season classification does not necessarily determine a lower value of ANPP $(\mathrm{F}=0.97, \mathrm{P}$ $=0.325)$. However, the previous year's growing season classification appears to have a stronger relation to ANPP than the same year growing season drought classification (Table 10).

Table 10. ANOVA and ANCOVA results for each of the three hypotheses examined.

\begin{tabular}{|c|c|c|c|c|}
\hline \multicolumn{3}{|c|}{ Hypothesis } & ANCOVA & ANOVA \\
\hline \multicolumn{3}{|c|}{ Hypothesis I (response=ANPP; variate=WS age) } & & 0.02 \\
\hline \multirow{4}{*}{$\begin{array}{c}\text { Hypothesis II } \\
\text { (response=ANPP; } \\
\text { variate=drought; } \\
\text { covariate=WS age) }\end{array}$} & \multirow[t]{2}{*}{$\begin{array}{c}\text { Same year } \\
\text { classification }\end{array}$} & $\begin{array}{l}\text { Annual } \\
\text { precip. }\end{array}$ & $2.72 * *$ & \\
\hline & & G.S. precip. & 1.75 & \\
\hline & \multirow[t]{2}{*}{$\begin{array}{l}\text { Previous year } \\
\text { classification }\end{array}$} & $\begin{array}{l}\text { Annual } \\
\text { precip. }\end{array}$ & 0.30 & \\
\hline & & G.S. precip. & $7.36^{*}$ & \\
\hline \multirow{4}{*}{$\begin{array}{c}\text { Hypothesis III } \\
\text { (response=ANPP; } \\
\text { variate=drought; } \\
\text { covariate=tree } \\
\text { species) }\end{array}$} & \multirow[t]{2}{*}{$\begin{array}{l}\text { Same year } \\
\text { classification }\end{array}$} & $\begin{array}{l}\text { Annual } \\
\text { precip. }\end{array}$ & 0.93 & \\
\hline & & G.S. precip. & 0.02 & \\
\hline & \multirow[t]{2}{*}{$\begin{array}{l}\text { Previous year } \\
\text { classification }\end{array}$} & $\begin{array}{l}\text { Annual } \\
\text { precip. }\end{array}$ & $2.70 * *$ & \\
\hline & & G.S. precip. & 0.97 & \\
\hline
\end{tabular}

* Significant at the $\alpha=0.05$.

** Significant at the $\alpha=0.10$. 


\section{DISCUSSION}

The mean ANPP for each plot indicated that over the whole time period examined (19712002), both watersheds had relatively equal rates of growth. However, upon closer examination of growth rate pattern(s) of each plot over the entire time period, year-by-year, the mature watershed demonstrated a leveled-off growth pattern that is expected of mature forested areas.

On the other hand, the younger watershed demonstrated an increasing rate of growth throughout the entire time period examined, typical of recently disturbed forests. Therefore, the first 15 years of the younger watershed's re-vegetation produced growth rates lower than the mature watershed. The second 15 years produced growth rates that were greater than the mature watershed. The cause of this shift is due to the greater density of trees in the younger watershed trumping the rate of growth of the mature watershed. Prior to the shift, when the mature watershed had a higher rate of growth, the much greater height and girth of the mature trees compensated for the lower radial growth of measured. In other words, it is important to look at the forest in all three dimensions, length, width, and height, because a small radial growth measurement does not necessarily correspond to a low rate of growth in large trees. Therefore, while the overall mean and analysis of variance used to test this first hypothesis may have demonstrated a relatively equal ANPP, a closer examination of the temporal aspect of growth is just as important and needs to be taken into account.

The analysis of covariance of average annual ANPP with regards to age, annual drought classifications indicate that the same year drought classification does determine a lower value of ANPP, while growing season drought classifications did not significantly indicate a lower value of ANPP. However, the opposite occurs when examining the previous year drought classifications. In this case, annual drought classifications indicate that the previous year 
drought classification does not determine a lower value of ANPP, while growing season drought classifications indicated that the previous year drought classification does determine a lower value of ANPP, and thus is a much better indicator of future values of aboveground ANPP. This type of time-lag forecasting in tree growth has been found to exist up to six years (Orwig and Abrams, 1997), thus a significant drought year may also continue to dampen the ANPP of an individual tree or forested area for a number of years following the event.

Similar work has shown that July PHDI (Palmer Hydrological Drought Index) correlated better with tree growth than annual precipitation (Cleaveland and Duvick, 1992). This study demonstrated the opposite for ANPP. I found that the annual drought classification was a better indicator of future productivity than growing season drought classification. The most likely reason for the results in this study to be different than other studies is inverse-distance weighting of PDSI measurements for cell 135, located $71 \mathrm{~km}$ from the Fernow Experimental Forest (Cook et al., 1999). Therefore, areas included in cell 135 PDSI measurements were low-land areas near the Ohio river and on the western, wetter, side of the mountains, which have a distinctly different climate than the mountainous region where the Fernow Experimental Forest is located.

The analysis of covariance of mean annual ANPP with regards to species, annual drought classification, and growing season drought classifications indicated that the previous year drought classification is a better indicator of future values of ANPP, as opposed to the same year drought classification. Thus, a time-lag response mechanism also appears to exist among the four most common species in the two watersheds. More importantly, it is most likely that previous studies that found that birch species are more affected by drought than maple species are supported by this study (Brenneman et al., 1978). Therefore species' preference with regards to moist habitats or dry habitats does play a significant role. 
Pearson correlations demonstrated variable relationships between precipitation and ANPP. While certain plots did show a significant negative relationship between the amount of precipitation received and ANPP, overall, the correlations did not demonstrate a viable relationship between precipitation and ANPP, thus the correlations suggest a need to examine other possible factors that play a role in the annual ANPP of forested areas. These other factors may include the size of the individual trees, the number of individual trees, sunlight availability, species composition, and nutrient availability, which are discussed in more detail with regards to basal area (Gilliam et al., 1995; MacDonald, 2003). However, previous studies have shown few differences in nutrient availability among both watersheds (Gilliam et al., 1994). For example, one explanation for the unusual negative correlations of the young watershed's plots with PDSI is the high number of birch (BELE) found in the young watershed and absence of birch trees in the mature watershed. Birch prefer drier habitat, therefore a lack of precipitation or lower PDSI value may lead to increased growth of birch. Any one, or a combination, of these factors is a possible explanation for the unusual negative correlations of the young watershed's plots with regards to PDSI and precipitation.

The basal area calculations were able to answer many of the questions concerning the variations of ANPP among the individual plots. The three main factors appear to be: 1) the size of the trees, 2) the surrounding environment, and 3) the number of individuals, or density. Upon examining individual plots in watershed 13, plot 1306 demonstrated a higher rate in overall ANPP each year, for the entire time period examined. This substantial difference was more compelling due to the fact that plot 1306 and plot 1301 are found at similar elevations in the watershed (both along the ridge-top). However, when examining the basal area of the plots in watershed 13, and specifically 1301 and 1306 , tree size is shown to play an important role in the 
annual ANPP. Thus, the ANPP of individual plots can be greatly affected by the size of the individual trees found at each plot. The second main factor, the surrounding environment, is better demonstrated in watershed 7 . The surrounding environments were classified into 1) valley, 2) ridge-top, and 3) mid-slope (Table 3). The valley environment exhibited the lowest annual ANPP among all plots, demonstrated by plot 702 (Figure 6). The ridge-top environment, oddly, exhibited a median annual ANPP among all plots, demonstrated by plot 701. The midslope environment exhibited the highest annual ANPP among all plots, demonstrated by plots 704, 705, and 706. The final main factor, the number of individuals, is also better demonstrated in watershed 7. Plots 701 and 703 have equivalent numbers of individuals as well as relatively equivalent basal areas, however, plots 704 and 706 also have equivalent, but larger, numbers of individuals as well as relatively equivalent, but larger, basal areas.

One of the most surprising findings is the relatively quick rebound, of forested areas that have been clear-cut and then allowed to re-grow. This was demonstrated by the younger watershed, which shows that a clear-cut forest appears to re-vegetate relatively quickly, on the order of 15 years, and thus, approach values, approximately $10,000 \mathrm{~kg} / \mathrm{ha} / \mathrm{year}$, seen in the mature watershed and at Oak Ridge and Hubbard Brook (Cole and Rapp, 1981). However, while the younger watershed did return to mature levels of annual ANPP found in the mature watershed, it quickly surpassed those values, and continued to grow at an accelerated rate. Therefore, the younger watershed has yet to reach the tipping point of growth and level off at mature watershed's rates. Furthermore, the younger watershed should not be called or even considered a mature watershed, due to the younger watershed having twice the density of the mature watershed in order to reach the corresponding level of annual ANPP and then surpass that level. Thus, the younger watershed is still several decades from becoming as mature a forest in 
terms of forest structure. Future expectations of the younger watershed would include thinning of weaker trees and the continual growth of the stronger trees creating a lower rate of growth, and ANPP, that will correspond to the levels seen in the mature watershed.

Overall litterfall calculations of this study revealed values that did not closely relate to values seen in temperate deciduous forests studied by Cole and Rapp (1981). Fernow Experimental Forest litterfall values corresponded more closely to boreal deciduous forest litterfall values. One possible explanation of this difference is the higher elevation of the Fernow Experimental forest, thus producing a cooler climate. A further aspect of this study was the examination of the allometric equations produced by Brenneman et al. (1978). In order to produce their equations, Brenneman (1978) used an across-the-board 5\% of the green bole aboveground biomass for all litterfall weights. My own biomass calculations and litterfall weight, suggest that $5 \%$ is not an acceptable amount to use for litterfall weights with regards to total biomass. A more reliable amount for litterfall weight use with regards to total biomass would be $2-5 \%$ for mature forests and $12-15 \%$ for young forests. This difference in litterfall weights between mature watersheds and young watersheds, as well as Brenneman's examination of only mature trees (37-80 years in age with a mean age of 63 years) produced relatively correct total biomass (and ANPP) values for the mature watershed, but substantially overestimated the values for the total biomass (and ANPP) of the young watershed when Brenneman's equations were used.

A small number of difficulties in field work and laboratory preparation/work, such as sample preparation and litterfall collection, may have led to errors that influenced my results. The greatest difficulty was the possible dating errors that were a result of the lack of crossdating of the increment cores. This lack of crossdating may have let a year shift occur among some of 
the increment cores where a possible false ring or missing ring was located. A related problem associated with the lack of crossdating cores was the subsequent removal of all dead trees. Each of these dead trees did contribute to ANPP but this contribution was removed from my estimates due to the inability to date the core. Thus, due to the absence of dead trees, the ANPP was likely underestimated to a minor degree; however, the overall pattern over time is accurate. However, this problem would not have affected biomass estimates and the comparison to Brenneman et al.'s (1978) work, previously discussed. Another weakness of this work is the exclusion of all belowground factors with regards to NPP. While large carbon pools and sequestration was shown to exist aboveground, there may be several belowground factors, such as belowground growth, respiration, and root death that offset the aboveground benefits of large carbon pools and sequestration. However, comparable carbon net uptake amounts found at the Harvard Forest, demonstrated that $70 \%$ of the mean ecosystem net uptake occurred aboveground, which is typical for eastern deciduous forests (Barford et al., 2001). Finally, one variable that has been used in the past that was not directly included in this study was temperature. Pan et al. (1997) concluded that air temperature is as important as precipitation in its influence on growth of most species in the Fernow Experimental Forest. The exclusion of this factor may very well have been associated with the anomalies, such as the unusual patterns at plot 1305 and 702 , found at some of the plots. However, temperature was indirectly included though the use of PDSI data. Furthermore, all of the previous results create a problem when looked at together. The correlations tend to show that precipitation is not a significant variable with regards to ANPP. On the other hand, ANOVA and ANCOVA results demonstrate that there is some significance with regards to the present year and future year ANPP. Previous work has shown that both 
methods of analyzing data, correlations and ANOVA/ANCOVA, are valid, therefore, creating a fundamental difficulty in interpreting the results of this study.

It appears that productivity in these forests is only weakly sensitive to climatic variation. While both watersheds show a weak relationship with precipitation, this relationship does not appear in the younger watershed until after 15 years. Thus, a younger watershed's growth curve is more important to the total annual aboveground productivity than interannual climatic variability. Also, based on this study's aspect and topographic position, it is likely that the surrounding environment and related factors play a more important role in determining aboveground productivity than climatic variation.

This is an important step forward from previous studies, such as Orwig and Abrams (1997), which examined several similar tree species in Virginia, and found a strong association between precipitation, temperature, PDSI, and ring widths. Similar results were obtained with regards to a time-lag mechanism between precipitation and future radial growth (ANPP in this study). A similar time-lag mechanism was also found by Pan et al. (1997) in the Fernow Experimental Forest, which demonstrated that variations in precipitation in the previous year is more important to tree growth than variation in precipitation in the current year. In both of these studies, only one relatively even-aged mature stand was used; therefore, one step forward in this study was the inclusion of two stand ages, young and mature. This allowed for the study of age effects in ANPP, drought response, and species, which was found to be significant.

In conclusion, while climatic variability plays a part in ANPP only when drought events are substantial, future productivity of temperate deciduous forests will most likely rely more heavily on activities such as land use and management that directly affect the age and structure of these forests. My research demonstrates that a lower rate of productivity among young 
watersheds is the norm in the first 15 years of re-vegetation. The difficult decision that must be made is to determine the optimal cutting interval that allows the maximum amount of growth coupled with the economic benefits of the timber industry. With regards to ecological benefits, this study suggests that a minimum of $30+$ years is required for a young watershed to approach the productivity rate of a mature watershed, however, while the productivity may be relatively equivalent, the younger trees are still too immature and have yet to reach a leveled-off growth rate. Therefore, the next step in this line of research is the study of tree stands/watersheds that are closer in age, such as 60 years and 90 years, to determine if at that time, the younger watershed has reached a leveled-off growth rate and can be considered suitable for timber harvest without excessive carbon losses. 


\section{REFERENCES CITED}

Abrams, M. D., D. A. Orwig, and M. J. Dockry. 1997. Dendroecology and successional status of two contrasting old-growth oak forests in the Blue Ridge Mountains, U.S.A. Canadian Journal of Forest Research 27:994-1002.

Adams, M. B., J. N. Kochenderfer, F. Wood, T. R. Angradi, and P. Edwards. 1994. Forty Years of Hydrometeorological Data from the Fernow Experimental Forest, West Virginia. General Technical Report NE-184.

Adams, M. B., J. N. Kochenderfer, T. R. Angradi, and P. J. Edwards. 1995. Nutrient budgets of two watersheds on the Fernow Experimental Forest. In: Gottschalk, K. W., S. L. C. Fosbroke, eds. Proceedings, 10th Central Hardwood Forest Conference, 1995 March 5-8, Morgantown, West Virginia. General Technical Report NE-197. Radnor, Pennsylvania: United States Department of Agriculture, Forest Service, NEFES: 119130.

Alley, W. M. 1984. The Palmer drought severity index: limitations and assumptions. Journal of Climate and Applied Meteorology 23:1100-1109.

Barford, C. C., S. C. Wofsy, M. L. Goulden, J. W. Munger, E. H. Pyle, S. P. Urbanski, L. Hutyra, S. R. Saleska, D. Firzjarrald, K. Moore. 2001. Factors controlling long- and short-term sequestration of atmospheric $\mathrm{CO}_{2}$ in a mid-latitude forest. Science 294:16881691.

Brenneman, B. B., D. J. Frederick, W. E. Gardner, L. H. Schoenhofen, and P. L. Marsh. 1978. Biomass of species and stands of West Virginia hardwoods. Presented at: The Central Hardwood Forest Conference II, Purdue University, West Lafayette, Indiana, November 16, 1978.

Carey, E. V., A. Sala, R. Keane, and R. M. Callaway. 2001. Are old forests underestimated as global carbon sinks? Global Change Biology 7:339-344.

Ciais, P., P. Peylin, and P. Bousquet. 2000. Regional biospheric carbon fluxes as inferred from atmospheric $\mathrm{CO}_{2}$ measurements. Ecological Applications 10:1574-1589.

Clark, D. A., S. Brown, D. W. Klicklighter, J. Q. Chambers, J. R. Thomlinson, and J. Ni. 2001. Measuring net primary production in forests: concepts and field methods. Ecological Applications 11:356-370.

Cleaveland, M. K. and D. N. Duvick. 1992. Iowa climate reconstructed from tree rings, 16401982. Water Resources Research 28:2607-2615. 
Clinton, B. D., K. J. Elliott, and W. T. Swank. 1997. Response of planted eastern white pine (Pinus strobus L.) to mechanical release, competition, and drought in the southern Appalachians. Southern Journal for Appalachian Forestry 21:19-23.

Cohen, W. B. and M. E. Harmon. 1996. Two decades of carbon flux from forests of the Pacific Northwest. BioScience 46: 836-844.

Cole, D. and M. Rapp. 1981. Elemental cycling in forest ecosystems. In: D. Reichle ed., Dynamic properties of forest ecosystems. Cambridge University Press. International Biological Programme 23: 341-409.

Cook, E. R., D. M. Meko, D. W. Stahle, and M. K. Cleaveland. 1999. Drought reconstructions for the continental United States. Journal of Climate 12:1145-1162.

DeLucia, E. H., J. G. Hamilton, S. L. Naidu, R. B. Thomas, J. A. Andrews, A. Finzi, M. Lavine, R. Matamala, J. E. Mohan, G. R. Hendrey, and W. H. Schlesinger. 1999. Net primary production of a forest ecosystem with experimental $\mathrm{CO}_{2}$ enrichment. Science 284:11771179.

Fernow Experimental Forest. 2003. United States Department of Agriculture Forest Service, Timber and Watershed Lab, P.O. Box 404, Parsons, West Virginia 26287, Phone: (304) 478-2000, Fax: (304) 478-8692. http://www.fs.fed.us/ne/parsons/fefhome.htm.

Fritts, H. C. 2001. Tree Rings and Climate. The Blackburn Press, Caldwell, New Jersey, USA.

Gilliam, F. S., N. L. Turrill, S. d. Aulick, D. K. Evans, and M. B. Adams. 1994. Herbaceous layer and soil response to experimental acidification in a central Appalachian hardwood forest. Journal of Environmental Quality 23:835-844.

Gilliam, F. S., N. L. Turrill, and M. B. Adams. 1995. Herbaceous-layer and overstory species in clear-cut and mature central Appalachian hardwood forests. Ecological Applications 5:947-955.

Goodale, C. L., M. J. Apps, R. A. Birdsey, C. B. Field, L. S. Heath, R. A. Houghton, J. C. Jenkins, G. H. Kohlmaier, W. Kurz, S. Liu, G.-J. Nabuurs, S. Nilsson, and A. Z. Shvidenko. 2002. Forest carbon sinks in the northern hemisphere. Ecological Applications 12:891-899.

Goulden, M. L., J. W. Munger, S. M. Fan, B. C. Daube, and S. C. Wofsy. 1996. Exchange of carbon dioxide by a deciduous forest: response to interannual climate variability. Science 271:1576-1578.

Hall, D. O., J. M. O. Scurlock, H. R. Bolhàr-Nordenkampf, R. C. Leegood, and S. P. Long. 1993. Photosynthesis and Production in a Changing Environment: A Field and Laboratory Manual, $1^{\text {st }}$ edition. Chapman and Hall, London, Great Britain. 
Harmon, M. E. 2001. Carbon sequestration in forests: addressing the scale question. Journal of Forestry 99:24-29.

IPCC. 2001. Climate Change 2001: The Scientific Basis. Wembley, U. K.

Law, B. E., P. E. Thorton, J. Irvine, P. M. Anthoni, and S. V. Tuyl. 2001. Carbon storage and fluxes in ponderosa pine forests at different developmental stages. Global Change Biology 7: 755-777.

Liu, Y. and R. N. Muller. 1993. Effect of drought and frost on radial growth of overstory and understory stems in a deciduous forest. The American Midland Naturalist 129:19-25.

MacDonald, G. 2003. Biogeography: introduction to space, time, and life. John Wiley and Sons, Inc., New York, New York, USA.

Martin, A. J. 1981. Taper and volume equations for selected Appalachian hardwood species. Research Paper NE-490.

Moffat, A. S. 1998. Temperate forests gain ground. Science 282:1253.

Newbould, P. J. 1967. Methods for Estimating the Primary Production of Forests. William Brothers Limited, Birkenhead, Great Britain.

Odum, E. P. 1969. The strategy of ecosystem development. Science 164:262-270.

Odum, E. P. 1971. Fundamentals of Ecology. W. B. Saunders Company, Philadelphia, Pennsylvania, USA.

Orwig, D. A. and M. D. Abrams. 1997. Variation in radial growth responses to drought among species, site, and canopy strata. Trees 11:474-484.

Pan, C., S. J. Tajchman, and J. N. Kochenderfer. 1997. Dendroclimatological analysis of major forest species of the central Appalachians. Forest Ecology and Management 98:77-87.

Palmer, W. C. 1965. Meteorologic drought. U. S. Weather Bureau, Research Paper No. 45, $58 \mathrm{pp}$.

Payette, S., M. J. Fortin, and C. Morneau. 1996. The recent sugar maple decline in southern Quebec: probable causes deduced from tree rings. Canadian Journal of Forest Research 26:1069-1078.

Phipps, R. L. 1985. Collecting, preparing, crossdating, and measuring tree increment cores. Water-Resources Investigations Report 85-4148.

Schimel, D., J. Melillo, H. Tian, A. D. McGuire, D. Kicklighter, T. Kittel, N. Rosenbloom, S. Running, P. Thornton, D. Ojima, W. Parton, R. Kelly, M. Sykes, R. Neilson, and B. 
Rizzo. 2000. Contribution of increasing $\mathrm{CO}_{2}$ and climate to carbon storage by ecosystems in the United States. Science 287:2004-2006.

Schlesinger, W. H. 1997. Biogeochemistry: An Analysis of Global Change. Academic Press, San Diego, California, USA.

Schuler, T. M. and A. R. Gillespie. 2000. Temporal patterns of woody species diversity in a central Appalachian forest from 1865 to 1997 . Journal of the Torrey Botanical Society 127:149-161.

Schweingruber, F. H. 1993. Trees and Wood in Dendrochronology. Springer-Verlag Berlin Heidelberg, Berlin, Germany.

Stokes, M. A. and T. L. Smiley. 1996. An Introduction to Tree-Ring Dating. The University of Arizona Press, Tucson, Arizona, USA.

Weather Forecast Office Pittsburgh. 2002. National Weather Service, William Drzal, 192 Shafer Road, Moon Township, Pennsylvania 15108, Phone: (412) 262-1591. http://www.drought.unl.edu/dm/index.html. http://206.156.54.206/er/pit/hydro.htm. william.drzal@noaa.gov. 
APPENDIX A

Tree Species and Abbreviations

\begin{tabular}{|c|c|c|}
\hline Common name & Scientific name & Abbreviation \\
\hline Basswood & Tilia americana & TIAM \\
\hline Beech & Fagus grandifolia & FAGR \\
\hline Birch & Betula lenta & BELE \\
\hline Black cherry & Prunus serotina & PRSE \\
\hline Black gum & Nyssa sylvatica & NYSY \\
\hline Black locust & Robinia pseudoacacia & ROPS \\
\hline Chestnut oak & Quercus prinus & QUPR \\
\hline Cucumber magnolia & Magnolia acuminata & MAAC \\
\hline Dotted thorn & Crataegus punctata & CRPU \\
\hline Frazer magnolia & Magnolia fraseri & MAFR \\
\hline Hickory & Carya cordiformis & CACO \\
\hline Red maple & Acer rubrum & ACRU \\
\hline Red oak & Quercus rubra & QURU \\
\hline Sassafras & Sassafras albidum & SAAL \\
\hline Scarlet oak & Quercus coccinea & QUCO \\
\hline Spicebush & Lindera benzoin & LIBE \\
\hline Striped maple & Acer pensylvanicum & ACPE \\
\hline Sugar maple & Acer saccharum & ACSA \\
\hline Tulip poplar & Liriodendron tulipifera & LITU \\
\hline Umbrella & Magnolia tripetala & MATR \\
\hline Unk./misc. & & --- \\
\hline White ash & Fraxinus americana & FRAM \\
\hline White oak & Quercus alba & QUAL \\
\hline Witchhazel & Hamamelis virginiana & HAVI \\
\hline
\end{tabular}




\section{APPENDIX B}

Tree Biomass Allometric Equations

\begin{tabular}{|c|c|c|}
\hline Species & Allometric equation & Diameter Range \\
\hline ACSA & $\mathrm{Wt}=2.4439 \mathrm{dbh}^{2.5735}$ & $5-50 \mathrm{~cm}$ \\
\hline BELE & $\mathrm{Wt}=1.6542 \mathrm{dbh}^{2.6606}$ & $5-50 \mathrm{~cm}$ \\
\hline LITU & $\mathrm{Wt}=1.0259 \mathrm{dbh}^{2.7324}$ & $5-50 \mathrm{~cm}$ \\
\hline FAGR & $\mathrm{Wt}=2.0394 \mathrm{dbh}^{2.5715}$ & $5-50 \mathrm{~cm}$ \\
\hline PRSE & $\mathrm{Wt}=1.8082 \mathrm{dbh}^{2.6174}$ & $5-50 \mathrm{~cm}$ \\
\hline QUPR & $\mathrm{Wt}=1.5509 \mathrm{dbh}^{2.7276}$ & $5-50 \mathrm{~cm}$ \\
\hline CACO & $\mathrm{Wt}=2.0340 \mathrm{dbh}^{2.6349}$ & $5-50 \mathrm{~cm}$ \\
\hline ACRU & $\mathrm{Wt}=2.0772 \mathrm{dbh}^{2.5080}$ & $5-50 \mathrm{~cm}$ \\
\hline QURU & $\mathrm{Wt}=2.4601 \mathrm{dbh}^{2.4572}$ & $5-50 \mathrm{~cm}$ \\
\hline QUCO & $\mathrm{Wt}=2.65743 \mathrm{dbh}^{2.43948}$ & $5-40 \mathrm{~cm}$ \\
\hline FRAM & $\mathrm{Wt}=2.3626 \mathrm{dbh}^{2.4798}$ & $5-50 \mathrm{~cm}$ \\
\hline QUAL & $\mathrm{Wt}=1.5647 \mathrm{dbh}^{2.6887}$ & $5-50 \mathrm{~cm}$ \\
\hline Hardwood & $\mathrm{LN} \mathrm{Wt}=0.95595+2.42640 \mathrm{LNdbh}$ & $2.5-25 \mathrm{~cm}$ \\
\hline
\end{tabular}

*(Brenneman et al., 1978).

**" $\mathrm{Wt}$ " is equal to the dry weights (in pounds) of aboveground forests biomass. 
APPENDIX C

Tree Species Frequency by Plot

\begin{tabular}{|l|l|l|l|l|l|l|l|l|l|l|l|l|}
\hline & $\mathbf{7 0 1}$ & $\mathbf{7 0 2}$ & $\mathbf{7 0 3}$ & $\mathbf{7 0 4}$ & $\mathbf{7 0 5}$ & $\mathbf{7 0 6}$ & $\mathbf{1 3 0 1}$ & $\mathbf{1 3 0 2}$ & $\mathbf{1 3 0 3}$ & $\mathbf{1 3 0 4}$ & $\mathbf{1 3 0 5}$ & $\mathbf{1 3 0 6}$ \\
\hline TIAM & 0 & 1 & 0 & 0 & 0 & 0 & 0 & 0 & 5 & 0 & 0 & 0 \\
\hline FAGR & 0 & 2 & 1 & 1 & 3 & 4 & 1 & 18 & 4 & 2 & 2 & 1 \\
\hline BELE & 20 & 3 & 3 & 10 & 10 & 15 & 0 & 0 & 0 & 0 & 0 & 0 \\
\hline PRSE & 3 & 1 & 0 & 18 & 1 & 7 & 0 & 0 & 0 & 0 & 0 & 0 \\
\hline NYSY & 0 & 0 & 0 & 0 & 0 & 0 & 0 & 0 & 0 & 1 & 0 & 0 \\
\hline ROPS & 9 & 1 & 0 & 0 & 0 & 2 & 0 & 0 & 0 & 0 & 0 & 0 \\
\hline QUPR & 0 & 0 & 0 & 0 & 0 & 0 & 0 & 1 & 0 & 0 & 0 & 0 \\
\hline MAAC & 0 & 0 & 0 & 0 & 0 & 0 & 0 & 0 & 1 & 0 & 1 & 0 \\
\hline CRPU & 0 & 4 & 0 & 0 & 0 & 0 & 0 & 0 & 0 & 0 & 0 & 0 \\
\hline MAFR & 0 & 0 & 1 & 0 & 0 & 0 & 0 & 0 & 0 & 0 & 0 & 0 \\
\hline CACO & 0 & 0 & 0 & 0 & 0 & 2 & 0 & 0 & 2 & 0 & 0 & 0 \\
\hline ACRU & 0 & 0 & 0 & 0 & 0 & 0 & 0 & 0 & 1 & 0 & 0 & 0 \\
\hline QURU & 0 & 0 & 4 & 2 & 0 & 2 & 0 & 2 & 0 & 4 & 1 & 7 \\
\hline SAAL & 0 & 0 & 0 & 0 & 0 & 8 & 0 & 0 & 0 & 0 & 0 & 0 \\
\hline QUCO & 0 & 0 & 0 & 0 & 0 & 0 & 0 & 0 & 0 & 1 & 0 & 0 \\
\hline ACPE & 0 & 1 & 0 & 0 & 0 & 0 & 0 & 0 & 0 & 0 & 0 & 0 \\
\hline ACSA & 0 & 5 & 8 & 5 & 2 & 3 & 12 & 1 & 4 & 14 & 12 & 7 \\
\hline LITU & 6 & 3 & 14 & 1 & 14 & 0 & 0 & 0 & 1 & 0 & 0 & 0 \\
\hline MATR & 0 & 0 & 0 & 1 & 0 & 0 & 0 & 0 & 0 & 3 & 0 & 0 \\
\hline FRAM & 0 & 0 & 0 & 0 & 0 & 5 & 0 & 0 & 0 & 0 & 0 & 0 \\
\hline QUAL & 0 & 0 & 0 & 0 & 0 & 0 & 0 & 0 & 0 & 0 & 0 & 1 \\
\hline Total & 38 & 21 & 31 & 38 & 30 & 48 & 13 & 22 & 18 & 25 & 16 & 16 \\
\hline
\end{tabular}


APPENDIX D

Litterfall Weights (g) per Species per Plot

\begin{tabular}{|l|l|l|l|l|l|l|l|l|l|l|l|l|}
\hline & $\mathbf{7 0 1}$ & $\mathbf{7 0 2}$ & $\mathbf{7 0 3}$ & $\mathbf{7 0 4}$ & $\mathbf{7 0 5}$ & $\mathbf{7 0 6}$ & $\mathbf{1 3 0 1}$ & $\mathbf{1 3 0 2}$ & $\mathbf{1 3 0 3}$ & $\mathbf{1 3 0 4}$ & $\mathbf{1 3 0 5}$ & $\mathbf{1 3 0 6}$ \\
\hline TIAM & 0 & 1.2 & 0 & 0 & 0 & 0 & 0 & 0 & 41.6 & 0 & 0 & 0 \\
\hline FAGR & 0 & 1.0 & 0.5 & 0 & 0 & 8.7 & 6.0 & 14.5 & 16.8 & 12.0 & 1.9 & 1.6 \\
\hline BELE & 36.6 & 10.1 & 2.4 & 17.7 & 12.3 & 35.1 & 8.1 & 1.0 & 12.0 & 1.5 & 17.1 & 2.3 \\
\hline PRSE & 40.1 & 46.3 & 0.9 & 74.9 & 11.4 & 29.8 & 4.1 & 0 & 0 & 0 & 0.9 & 0.6 \\
\hline NYSY & 0 & 0 & 0 & 0 & 0 & 0 & 0 & 0 & 0 & 9.9 & 0 & 0 \\
\hline ROPS & 25.3 & 6.2 & 0 & 0 & 0 & 1.7 & 0 & 0 & 0 & 0 & 0 & 0 \\
\hline QUPR & 0 & 0 & 0 & 0 & 0 & 0 & 13.8 & 7.0 & 0 & 0 & 4.0 & 0 \\
\hline MAAC & 0 & 0 & 0 & 0 & 0 & 0 & 0 & 0 & 6.2 & 0 & 10.7 & 0 \\
\hline CRPU & 0 & 0.7 & 0 & 0 & 0 & 0 & 0 & 0 & 0 & 0 & 0 & 0 \\
\hline MAFR & 0 & 0 & 0 & 0 & 0 & 0 & 0 & 0 & 0 & 12.3 & 0 & 0 \\
\hline CACO & 0 & 0 & 0 & 0 & 0 & 3.2 & 0 & 0 & 5.6 & 0 & 0 & 0 \\
\hline ACRU & 0 & 0 & 0 & 2.3 & 0 & 0 & 0 & 0 & 0 & 0 & 0.6 & 7.7 \\
\hline QURU & 0 & 0 & 3.6 & 2.7 & 1.0 & 3.0 & 7.1 & 35.8 & 26.4 & 44.7 & 73.5 & 40.1 \\
\hline SAAL & 0 & 0 & 0 & 0 & 0 & 9.5 & 0 & 0 & 0 & 0 & 0 & 0 \\
\hline QUCO & 1.7 & 0 & 0 & 0 & 0 & 0 & 12.8 & 0 & 1.5 & 29.8 & 0 & 0 \\
\hline LIBE & 0 & 0 & 0 & 0 & 0 & 0 & 0 & 0 & 0 & 0 & 0 & 0 \\
\hline ACPE & 0 & 3.4 & 0 & 0 & 0 & 0 & 0 & 0 & 0 & 0 & 8.0 & 0 \\
\hline ACSA & 7.7 & 44.3 & 2.2 & 5.9 & 3.2 & 12.0 & 84.2 & 0.5 & 20.4 & 31.4 & 29.8 & 21.7 \\
\hline LITU & 15.1 & 17.4 & 33.7 & 9.6 & 17.7 & 5.5 & 0 & 1.1 & 14.6 & 0 & 3.9 & 1.1 \\
\hline MATR & 0 & 0 & 0 & 1.8 & 0 & 0 & 0 & 0 & 0 & 0.5 & 0 & 0 \\
\hline FRAM & 0 & 0 & 0 & 0 & 0 & 0.7 & 0 & 0 & 0 & 0 & 0 & 0 \\
\hline QUAL & 0 & 0 & 0 & 0 & 0 & 0 & 0 & 0 & 0 & 0 & 0 & 13.6 \\
\hline HAVI & 0 & 0 & 0 & 0 & 0 & 0 & 0 & 0 & 0 & 1.2 & 0 & 0 \\
\hline Unk./misc. & 37.9 & 53.8 & 21.6 & 49.3 & 23.5 & 39.0 & 51.9 & 22.3 & 30.9 & 26.6 & 46.4 & 67.8 \\
\hline Total** & 3288 & 3688 & $1298^{*}$ & 3284 & 1382 & 2964 & 3760 & 1644 & 3520 & 3398 & 3936 & 3130 \\
\hline
\end{tabular}

* Three weeks of litterfall data during the fall was lost due to stolen collection baskets.

** Totals are calculated estimates of litterfall expressed in $\mathrm{kg} / \mathrm{ha} / \mathrm{year}$. 


\section{APPENDIX E}

Bark Coefficients*

\begin{tabular}{|c|c|}
\hline Tree Species & Bark Coeffiecents $\mathbf{( \mathbf { D B H } _ { \mathbf { i b } }} \mathbf{D B H}_{\mathbf{o b}} \mathbf{)} * *$ \\
\hline TIAM & 0.907 \\
\hline FAGR & 0.968 \\
\hline BELE & 0.939 \\
\hline PRSE & 0.923 \\
\hline ROPS & 0.861 \\
\hline QUPR & 0.887 \\
\hline MAAC & 0.912 \\
\hline CACO & 0.915 \\
\hline ACRU & 0.942 \\
\hline QURU & 0.921 \\
\hline QUCO & 0.926 \\
\hline ACSA & 0.942 \\
\hline LITU & 0.896 \\
\hline FRAM & 0.913 \\
\hline QUAL & 0.929 \\
\hline All species & 0.918 \\
\hline
\end{tabular}

*(Martin, 1981).

** "ib" is inside of bark and "ob" is outside of bark. 
APPENDIX F

Tree Survey and Measurements

\begin{tabular}{|c|c|c|c|c|c|}
\hline plot \# & tree \# & species & status & dbh (cm) & tree height (m) \\
\hline 701 & 1 & LITU & $\mathrm{L}$ & 26.0 & 18.72 \\
\hline 701 & 2 & ROPS & $\mathrm{L}$ & 22.8 & 20.16 \\
\hline 701 & 3 & LITU & $\mathrm{L}$ & 29.0 & 22.10 \\
\hline 701 & 4 & LITU & $\mathrm{L}$ & 21.4 & 20.32 \\
\hline 701 & 5 & BELE & $\mathrm{L}$ & 8.3 & 10.00 \\
\hline 701 & 6 & PRSE & $\mathrm{L}$ & 31.3 & 24.00 \\
\hline 701 & 7 & BELE & $\mathrm{L}$ & 9.4 & 10.00 \\
\hline 701 & 8 & BELE & $\mathrm{L}$ & 10.8 & 13.44 \\
\hline 701 & 9 & BELE & $\mathrm{L}$ & 9.5 & 13.44 \\
\hline 701 & 10 & BELE & $\mathrm{L}$ & 18.0 & 19.84 \\
\hline 701 & 11 & PRSE & $\mathrm{L}$ & 38.4 & 22.50 \\
\hline 701 & 12 & BELE & $\mathrm{L}$ & 7.6 & 13.65 \\
\hline 701 & 13 & ROPS & $\mathrm{L}$ & 21.4 & 16.80 \\
\hline 701 & 14 & ROPS & D & 8.5 & 8.00 \\
\hline 701 & 15 & BELE & $\mathrm{L}$ & 10.1 & 14.10 \\
\hline 701 & 16 & ROPS & $\mathrm{L}$ & 26.0 & 23.00 \\
\hline 701 & 17 & ROPS & D & 12.1 & 10.00 \\
\hline 701 & 18 & PRSE & $\mathrm{L}$ & 15.1 & 16.05 \\
\hline 701 & 19 & BELE & $\mathrm{L}$ & 8.2 & 11.88 \\
\hline 701 & 20 & BELE & $\mathrm{L}$ & 9.5 & 12.60 \\
\hline 701 & 21 & ROPS & $\mathrm{L}$ & 21.9 & 18.70 \\
\hline 701 & 22 & BELE & $\mathrm{L}$ & 12.8 & 14.00 \\
\hline 701 & 23 & BELE & $\mathrm{L}$ & 13.3 & 16.28 \\
\hline 701 & 24 & LITU & $\mathrm{L}$ & 18.8 & 17.67 \\
\hline 701 & 25 & ROPS & $\mathrm{L}$ & 17.9 & 23.10 \\
\hline 701 & 26 & BELE & D & 11.5 & 2.50 \\
\hline 701 & 27 & BELE & $\mathrm{L}$ & 11.0 & 13.13 \\
\hline 701 & 28 & BELE & $\mathrm{L}$ & 12.4 & 12.60 \\
\hline 701 & 29 & ROPS & $\mathrm{L}$ & 21.1 & 23.37 \\
\hline 701 & 30 & BELE & $\mathrm{L}$ & 7.2 & 11.04 \\
\hline 701 & 31 & BELE & $\mathrm{L}$ & 11.7 & 13.50 \\
\hline 701 & 32 & ROPS & $\mathrm{L}$ & 21.2 & 20.30 \\
\hline 701 & 33 & BELE & D & 5.6 & 1.50 \\
\hline 701 & 34 & BELE & $\mathrm{L}$ & 8.9 & 10.73 \\
\hline 701 & 35 & LITU & $\mathrm{L}$ & 26.6 & 16.75 \\
\hline 701 & 36 & ROPS & $\mathrm{L}$ & 22.7 & 21.13 \\
\hline 701 & 37 & BELE & D & 31.0 & 5.50 \\
\hline 701 & 38 & ROPS & $\mathrm{L}$ & 16.7 & 15.08 \\
\hline 701 & 39 & ROPS & D & 7.7 & 7.50 \\
\hline
\end{tabular}

\begin{tabular}{|c|c|c|c|c|c|}
\hline plot \# & tree \# & species & status & dbh (cm) & tree height (m) \\
\hline 701 & 40 & BELE & $\mathrm{L}$ & 9.3 & 15.50 \\
\hline 701 & 41 & LITU & $\mathrm{D}$ & 8.3 & 12.00 \\
\hline 701 & 42 & LITU & $\mathrm{L}$ & 7.9 & 13.44 \\
\hline 701 & 43 & BELE & $\mathrm{L}$ & 7.0 & 10.88 \\
\hline 701 & 44 & BELE & $\mathrm{L}$ & 10.4 & 14.00 \\
\hline 701 & 45 & BELE & $\mathrm{L}$ & 6.7 & 10.50 \\
\hline 702 & 1 & ACSA & $\mathrm{L}$ & 8.9 & 10.03 \\
\hline 702 & 2 & ACSA & $\mathrm{L}$ & 18.4 & 10.92 \\
\hline 702 & 3 & TIAM & $\mathrm{L}$ & 8.9 & 11.00 \\
\hline 702 & 4 & FAGR & $\mathrm{L}$ & 7.3 & 13.50 \\
\hline 702 & 5 & LITU & $\mathrm{L}$ & 7.6 & 7.50 \\
\hline 702 & 6 & ROPS & $\mathrm{L}$ & 6.7 & 10.00 \\
\hline 702 & 7 & CRPU & $\mathrm{L}$ & 7.2 & 7.15 \\
\hline 702 & 8 & CRPU & $\mathrm{L}$ & 9.0 & 5.98 \\
\hline 702 & 9 & CRPU & $\mathrm{L}$ & 9.6 & 9.36 \\
\hline 702 & 10 & $\mathrm{ACPE}$ & $\mathrm{D}$ & 14.0 & 8.97 \\
\hline 702 & 11 & $\mathrm{ACPE}$ & $\mathrm{L}$ & 34.1 & 18.85 \\
\hline 702 & 12 & FAGR & $\mathrm{D}$ & 20.3 & 6.50 \\
\hline 702 & 13 & FAGR & $\mathrm{L}$ & 13.5 & 11.40 \\
\hline 702 & 14 & FAGR & D & 19.7 & 8.50 \\
\hline 702 & 15 & CRPU & $\mathrm{L}$ & 6.2 & 8.00 \\
\hline 702 & 16 & ACSA & $\mathrm{L}$ & 6.8 & 7.50 \\
\hline 702 & 17 & ACSA & $\mathrm{L}$ & 6.9 & 10.50 \\
\hline 702 & 18 & ACSA & $\mathrm{L}$ & 9.3 & 10.50 \\
\hline 702 & 19 & BELE & $\mathrm{L}$ & 11.3 & 16.33 \\
\hline 702 & 20 & LITU & $\mathrm{L}$ & 31.6 & 21.87 \\
\hline 702 & 21 & BELE & $\mathrm{L}$ & 6.8 & 6.00 \\
\hline 702 & 22 & PRSE & $\mathrm{L}$ & 13.5 & 18.75 \\
\hline 702 & 23 & BELE & $\mathrm{L}$ & 19.8 & 20.16 \\
\hline 702 & 24 & LITU & $\mathrm{L}$ & 39.3 & 28.35 \\
\hline 703 & 1 & BELE & $\mathrm{L}$ & 5.7 & 9.50 \\
\hline 703 & 2 & LITU & $\mathrm{L}$ & 18.1 & 19.20 \\
\hline 703 & 3 & ACSA & $\mathrm{L}$ & 5.4 & 9.50 \\
\hline 703 & 4 & ACSA & $\mathrm{L}$ & 6.0 & 12.00 \\
\hline 703 & 5 & unk & $\mathrm{D}$ & 11.5 & 10.70 \\
\hline 703 & 6 & QURU & $\mathrm{L}$ & 6.5 & 10.68 \\
\hline 703 & 7 & QURU & $\mathrm{L}$ & 5.8 & 11.50 \\
\hline 703 & 8 & unk & D & 6.8 & 2.50 \\
\hline 703 & 9 & unk & D & 5.5 & 7.50 \\
\hline
\end{tabular}




\begin{tabular}{|c|c|c|c|c|c|}
\hline plot \# & tree \# & species & status & dbh $(\mathrm{cm})$ & tree height (m) \\
\hline 703 & 10 & LITU & $\mathrm{L}$ & 5.5 & 10.50 \\
\hline 703 & 11 & BELE & $\mathrm{L}$ & 6.4 & 11.50 \\
\hline 703 & 12 & ACSA & $\mathrm{L}$ & 6.1 & 12.00 \\
\hline 703 & 13 & unk & $\mathrm{D}$ & 8.2 & 10.50 \\
\hline 703 & 14 & ACSA & $\mathrm{L}$ & 5.7 & 9.72 \\
\hline 703 & 15 & ACSA & $\mathrm{L}$ & 6.4 & 9.41 \\
\hline 703 & 16 & unk & $\mathrm{D}$ & 9.3 & 7.94 \\
\hline 703 & 17 & unk & $\mathrm{D}$ & 11.9 & 11.27 \\
\hline 703 & 18 & unk & $\mathrm{D}$ & 10.4 & 11.27 \\
\hline 703 & 19 & QURU & $\mathrm{L}$ & 7.5 & 12.00 \\
\hline 703 & 20 & LITU & $\mathrm{L}$ & 43.2 & 26.25 \\
\hline 703 & 21 & unk & $\mathrm{D}$ & 5.9 & 2.50 \\
\hline 703 & 22 & LITU & $\mathrm{L}$ & 16.5 & 23.59 \\
\hline 703 & 23 & ACSA & $\mathrm{L}$ & 23.6 & 25.43 \\
\hline 703 & 24 & LITU & $\mathrm{L}$ & 26.6 & 23.60 \\
\hline 703 & 25 & BELE & $\mathrm{L}$ & 17.8 & 19.53 \\
\hline 703 & 26 & LITU & $\mathrm{L}$ & 27.6 & 22.62 \\
\hline 703 & 27 & LITU & $\mathrm{L}$ & 23.4 & 21.86 \\
\hline 703 & 28 & LITU & $\mathrm{L}$ & 19.5 & 22.36 \\
\hline 703 & 29 & LITU & $\mathrm{L}$ & 23.5 & 18.36 \\
\hline 703 & 30 & unk & $\mathrm{D}$ & 10.7 & 8.64 \\
\hline 703 & 31 & unk & D & 15.4 & 13.12 \\
\hline 703 & 32 & LITU & $\mathrm{L}$ & 16.7 & 23.20 \\
\hline 703 & 33 & FAGR & $\mathrm{L}$ & 24.7 & 23.97 \\
\hline 703 & 34 & QURU & $\mathrm{L}$ & 6.6 & 9.64 \\
\hline 703 & 35 & LITU & $\mathrm{L}$ & 39.6 & 26.09 \\
\hline 703 & 36 & unk & D & 8.6 & 12.50 \\
\hline 703 & 37 & LITU & $\mathrm{L}$ & 8.3 & 14.00 \\
\hline 703 & 38 & LITU & $\mathrm{L}$ & 20.0 & 23.29 \\
\hline 703 & 39 & ACSA & $\mathrm{L}$ & 11.3 & 14.24 \\
\hline 703 & 40 & LITU & $\mathrm{L}$ & 17.2 & 18.63 \\
\hline 703 & 41 & ACSA & $\mathrm{L}$ & 9.5 & 12.76 \\
\hline 703 & 42 & MAFR & $\mathrm{L}$ & 9.8 & 13.13 \\
\hline 704 & 1 & PRSE & $\mathrm{L}$ & 22.4 & 11.16 \\
\hline 704 & 2 & PRSE & $\mathrm{L}$ & 18.0 & 20.80 \\
\hline 704 & 3 & unk & D & 12.2 & 5.85 \\
\hline 704 & 4 & PRSE & $\mathrm{L}$ & 13.1 & 22.36 \\
\hline 704 & 5 & unk & D & 10.5 & 2.50 \\
\hline 704 & 6 & PRSE & $\mathrm{L}$ & 28.5 & 29.70 \\
\hline 704 & 7 & unk & D & 6.9 & 7.00 \\
\hline 704 & 8 & unk & D & 13.2 & 6.50 \\
\hline 704 & 9 & unk & $\mathrm{D}$ & 9.1 & 6.30 \\
\hline 704 & 10 & unk & D & 10.8 & 7.00 \\
\hline 704 & 11 & ACSA & $\mathrm{L}$ & 9.1 & 12.45 \\
\hline
\end{tabular}

\begin{tabular}{|c|c|c|c|c|c|}
\hline plot \# & tree \# & species & status & dbh (cm) & tree height (m) \\
\hline 704 & 12 & PRSE & $\mathrm{L}$ & 12.1 & 13.80 \\
\hline 704 & 13 & PRSE & $\mathrm{L}$ & 33.2 & 29.85 \\
\hline 704 & 14 & unk & $\mathrm{D}$ & 8.4 & 3.00 \\
\hline 704 & 15 & PRSE & $\mathrm{L}$ & 27.5 & 29.04 \\
\hline 704 & 16 & unk & $\mathrm{D}$ & 7.5 & 7.00 \\
\hline 704 & 17 & unk & $\mathrm{D}$ & 16.0 & 14.41 \\
\hline 704 & 18 & unk & $\mathrm{D}$ & 13.0 & 11.16 \\
\hline 704 & 19 & LITU & $\mathrm{L}$ & 28.9 & 29.05 \\
\hline 704 & 20 & BELE & $\mathrm{L}$ & 10.7 & 13.72 \\
\hline 704 & 21 & PRSE & $\mathrm{L}$ & 31.5 & 33.22 \\
\hline 704 & 22 & BELE & $\mathrm{L}$ & 15.5 & 20.39 \\
\hline 704 & 23 & unk & $\mathrm{D}$ & 6.0 & 2.50 \\
\hline 704 & 24 & PRSE & $\mathrm{L}$ & 25.3 & 27.80 \\
\hline 704 & 25 & PRSE & $\mathrm{L}$ & 13.0 & 15.60 \\
\hline 704 & 26 & PRSE & $\mathrm{L}$ & 9.7 & 13.59 \\
\hline 704 & 27 & unk & $\mathrm{D}$ & 8.9 & 10.00 \\
\hline 704 & 28 & FAGR & $\mathrm{L}$ & 6.3 & 10.00 \\
\hline 704 & 29 & unk & $\mathrm{D}$ & 9.6 & 8.25 \\
\hline 704 & 30 & unk & $\mathrm{D}$ & 7.9 & 7.25 \\
\hline 704 & 31 & ACSA & $\mathrm{L}$ & 5.7 & 9.02 \\
\hline 704 & 32 & MATR & $\mathrm{L}$ & 13.4 & 16.01 \\
\hline 704 & 33 & QURU & $\mathrm{L}$ & 9.3 & 11.70 \\
\hline 704 & 34 & LITU & $\mathrm{D}$ & 11.1 & 16.34 \\
\hline 704 & 35 & BELE & $\mathrm{L}$ & 17.3 & 25.48 \\
\hline 704 & 36 & QURU & $\mathrm{L}$ & 10.8 & 14.40 \\
\hline 704 & 37 & BELE & $\mathrm{L}$ & 16.4 & 18.69 \\
\hline 704 & 38 & unk & $\mathrm{D}$ & 10.1 & 14.84 \\
\hline 704 & 39 & PRSE & $\mathrm{L}$ & 29.1 & 23.87 \\
\hline 704 & 40 & BELE & $\mathrm{L}$ & 16.4 & 20.91 \\
\hline 704 & 41 & unk & $\mathrm{D}$ & 8.1 & 7.00 \\
\hline 704 & 42 & unk & $\mathrm{D}$ & 9.8 & 6.00 \\
\hline 704 & 43 & unk & $\mathrm{D}$ & 5.8 & 3.50 \\
\hline 704 & 44 & unk & $\mathrm{D}$ & 6.2 & 3.00 \\
\hline 704 & 46 & ACSA & $\mathrm{L}$ & 6.4 & 7.50 \\
\hline 704 & 47 & BELE & $\mathrm{L}$ & 12.1 & 31.08 \\
\hline 704 & 48 & BELE & $\mathrm{L}$ & 23.0 & 25.52 \\
\hline 704 & 49 & BELE & $\mathrm{L}$ & 11.4 & 18.73 \\
\hline 704 & 50 & BELE & $\mathrm{L}$ & 19.8 & 24.15 \\
\hline 704 & 51 & ACSA & $\mathrm{L}$ & 7.5 & 7.50 \\
\hline 704 & 52 & PRSE & $\mathrm{L}$ & 20.2 & 26.08 \\
\hline 704 & 53 & BELE & $\mathrm{D}$ & 8.0 & 6.50 \\
\hline 704 & 54 & BELE & $\mathrm{L}$ & 22.8 & 24.70 \\
\hline 704 & 55 & PRSE & $\mathrm{L}$ & 26.0 & 21.60 \\
\hline 704 & 56 & PRSE & $\mathrm{L}$ & 19.4 & 24.96 \\
\hline
\end{tabular}




\begin{tabular}{|c|c|c|c|c|c|}
\hline plot \# & tree \# & species & status & dbh (cm) & tree height (m) \\
\hline 704 & 57 & PRSE & $\mathrm{L}$ & 9.6 & 10.26 \\
\hline 704 & 58 & ACSA & $\mathrm{L}$ & 8.9 & 7.92 \\
\hline 704 & 59 & unk & $\mathrm{D}$ & 7.2 & 8.00 \\
\hline 704 & 60 & PRSE & $\mathrm{L}$ & 32.6 & 27.72 \\
\hline 704 & 62 & PRSE & $\mathrm{L}$ & 30.5 & 23.98 \\
\hline 705 & 1 & ACSA & $\mathrm{L}$ & 5.9 & 8.55 \\
\hline 705 & 2 & unk & $\mathrm{D}$ & 10.0 & 4.00 \\
\hline 705 & 3 & unk & $\mathrm{D}$ & 7.9 & 5.50 \\
\hline 705 & 4 & unk & $\mathrm{D}$ & 7.1 & 6.50 \\
\hline 705 & 5 & LITU & $\mathrm{L}$ & 24.6 & 26.52 \\
\hline 705 & 6 & LITU & $\mathrm{L}$ & 12.5 & 15.98 \\
\hline 705 & 7 & BELE & $\mathrm{L}$ & 24.9 & 23.69 \\
\hline 705 & 8 & LITU & $\mathrm{L}$ & 31.4 & 29.03 \\
\hline 705 & 9 & LITU & $\mathrm{L}$ & 27.1 & 24.19 \\
\hline 705 & 10 & BELE & $\mathrm{L}$ & 12.6 & 14.45 \\
\hline 705 & 11 & LITU & $\mathrm{L}$ & 38.7 & 25.67 \\
\hline 705 & 12 & BELE & $\mathrm{L}$ & 12.3 & 13.92 \\
\hline 705 & 13 & LITU & $\mathrm{L}$ & 15.9 & 16.17 \\
\hline 705 & 14 & unk & D & 5.2 & 5.61 \\
\hline 705 & 15 & unk & $\mathrm{D}$ & 12.5 & 14.28 \\
\hline 705 & 16 & LITU & $\mathrm{L}$ & 24.7 & 26.43 \\
\hline 705 & 17 & LITU & $\mathrm{L}$ & 29.8 & 25.30 \\
\hline 705 & 18 & BELE & $\mathrm{L}$ & 14.3 & 16.08 \\
\hline 705 & 19 & BELE & $\mathrm{L}$ & 11.8 & 16.99 \\
\hline 705 & 20 & BELE & $\mathrm{L}$ & 19.0 & 16.80 \\
\hline 705 & 21 & unk & $\mathrm{D}$ & 10.6 & 4.00 \\
\hline 705 & 22 & unk & $\mathrm{D}$ & 13.7 & 14.69 \\
\hline 705 & 23 & unk & $\mathrm{D}$ & 7.1 & 3.50 \\
\hline 705 & 24 & unk & $\mathrm{D}$ & 7.0 & 6.50 \\
\hline 705 & 25 & unk & $\mathrm{D}$ & 8.4 & 5.50 \\
\hline 705 & 26 & BELE & $\mathrm{L}$ & 18.8 & 18.04 \\
\hline 705 & 27 & LITU & $\mathrm{L}$ & 21.0 & 23.65 \\
\hline 705 & 28 & PRSE & $\mathrm{L}$ & 21.4 & 21.94 \\
\hline 705 & 29 & unk & $\mathrm{D}$ & 8.8 & 6.00 \\
\hline 705 & 30 & ACSA & $\mathrm{L}$ & 13.7 & 11.90 \\
\hline 705 & 31 & BELE & $\mathrm{L}$ & 18.3 & 22.68 \\
\hline 705 & 32 & BELE & $\mathrm{L}$ & 13.1 & 19.32 \\
\hline 705 & 33 & BELE & $\mathrm{L}$ & 16.4 & 19.74 \\
\hline 705 & 34 & FAGR & $\mathrm{L}$ & 8.0 & 10.64 \\
\hline 705 & 35 & LITU & $\mathrm{L}$ & 37.0 & 28.67 \\
\hline 705 & 36 & LITU & $\mathrm{L}$ & 13.9 & 19.75 \\
\hline 705 & 37 & LITU & $\mathrm{L}$ & 23.1 & 27.23 \\
\hline 705 & 38 & LITU & $\mathrm{L}$ & 16.2 & 20.01 \\
\hline 705 & 39 & FAGR & $\mathrm{L}$ & 5.9 & 6.08 \\
\hline
\end{tabular}

\begin{tabular}{|c|c|c|c|c|c|}
\hline plot \# & tree \# & species & status & dbh (cm) & tree height (m) \\
\hline 705 & 40 & LITU & $\mathrm{L}$ & 25.7 & 25.30 \\
\hline 705 & 41 & FAGR & $\mathrm{L}$ & 34.0 & 28.50 \\
\hline 706 & 1 & unk & $\mathrm{D}$ & 8.6 & 11.50 \\
\hline 706 & 2 & PRSE & $\mathrm{L}$ & 8.0 & 7.00 \\
\hline 706 & 3 & PRSE & $\mathrm{L}$ & 26.9 & 20.86 \\
\hline 706 & 4 & BELE & $\mathrm{L}$ & 12.5 & 18.76 \\
\hline 706 & 5 & PRSE & $\mathrm{L}$ & 28.5 & 26.60 \\
\hline 706 & 6 & QURU & $\mathrm{L}$ & 15.0 & 17.52 \\
\hline 706 & 7 & $\mathrm{CACO}$ & $\mathrm{L}$ & 14.0 & 19.00 \\
\hline 706 & 8 & ACSA & $\mathrm{L}$ & 5.5 & 9.00 \\
\hline 706 & 9 & PRSE & $\mathrm{L}$ & 31.2 & 28.68 \\
\hline 706 & 10 & unk & $\mathrm{D}$ & 22.0 & 26.04 \\
\hline 706 & 11 & $\mathrm{CACO}$ & $\mathrm{L}$ & 11.3 & 15.00 \\
\hline 706 & 12 & PRSE & $\mathrm{L}$ & 14.7 & 19.63 \\
\hline 706 & 13 & PRSE & $\mathrm{L}$ & 18.0 & 22.08 \\
\hline 706 & 14 & FRAM & $\mathrm{L}$ & 13.5 & 23.12 \\
\hline 706 & 15 & unk & $\mathrm{D}$ & 8.4 & 12.29 \\
\hline 706 & 16 & ROPS & $\mathrm{L}$ & 17.2 & 15.58 \\
\hline 706 & 17 & BELE & $\mathrm{L}$ & 7.1 & 12.42 \\
\hline 706 & 18 & FAGR & $\mathrm{L}$ & 24.2 & 22.20 \\
\hline 706 & 19 & BELE & $\mathrm{L}$ & 8.1 & 10.24 \\
\hline 706 & 20 & BELE & $\mathrm{L}$ & 23.3 & 21.25 \\
\hline 706 & 21 & QURU & $\mathrm{L}$ & 9.5 & 11.34 \\
\hline 706 & 22 & BELE & $\mathrm{L}$ & 18.6 & 20.01 \\
\hline 706 & 23 & BELE & $\mathrm{L}$ & 9.5 & 17.11 \\
\hline 706 & 24 & unk & $\mathrm{D}$ & 12.7 & 14.25 \\
\hline 706 & 25 & FAGR & $\mathrm{L}$ & 9.2 & 8.23 \\
\hline 706 & 26 & BELE & $\mathrm{L}$ & 16.3 & 17.34 \\
\hline 706 & 27 & BELE & $\mathrm{L}$ & 15.2 & 15.64 \\
\hline 706 & 28 & ROPS & $\mathrm{L}$ & 16.6 & 19.38 \\
\hline 706 & 29 & unk & $\mathrm{D}$ & 6.6 & 5.50 \\
\hline 706 & 30 & ACSA & $\mathrm{L}$ & 5.6 & 10.50 \\
\hline 706 & 31 & SAAL & $\mathrm{L}$ & 14.2 & 14.55 \\
\hline 706 & 32 & unk & $\mathrm{D}$ & 6.7 & 5.00 \\
\hline 706 & 33 & unk & $\mathrm{D}$ & 9.6 & 7.70 \\
\hline 706 & 34 & SAAL & $\mathrm{L}$ & 14.8 & 17.22 \\
\hline 706 & 35 & SAAL & $\mathrm{D}$ & 9.1 & 6.50 \\
\hline 706 & 36 & FRAM & $\mathrm{D}$ & 6.5 & 11.38 \\
\hline 706 & 37 & FRAM & $\mathrm{L}$ & 11.9 & 20.52 \\
\hline 706 & 38 & SAAL & $\mathrm{L}$ & 17.8 & 21.72 \\
\hline 706 & 39 & unk & $\mathrm{D}$ & 10.5 & 12.36 \\
\hline 706 & 40 & BELE & $\mathrm{L}$ & 6.7 & 12.00 \\
\hline 706 & 41 & FRAM & $\mathrm{L}$ & 16.6 & 20.17 \\
\hline 706 & 42 & SAAL & L & 12.8 & 16.8 \\
\hline
\end{tabular}




\begin{tabular}{|c|c|c|c|c|c|}
\hline plot \# & tree \# & species & status & dbh (cm) & tree height (m) \\
\hline 706 & 43 & SAAL & $\mathrm{L}$ & 13.0 & 20.21 \\
\hline 706 & 44 & SAAL & $\mathrm{L}$ & 15.4 & 20.58 \\
\hline 706 & 45 & SAAL & $\mathrm{L}$ & 18.1 & 18.92 \\
\hline 706 & 46 & SAAL & $\mathrm{L}$ & 15.8 & 21.20 \\
\hline 706 & 47 & SAAL & $\mathrm{D}$ & 10.5 & 13.32 \\
\hline 706 & 48 & unk & $\mathrm{D}$ & 7.6 & 10.50 \\
\hline 706 & 49 & unk & $\mathrm{D}$ & 10.5 & 2.50 \\
\hline 706 & 50 & SAAL & $\mathrm{D}$ & 9.7 & 15.00 \\
\hline 706 & 51 & BELE & $\mathrm{L}$ & 6.7 & 10.45 \\
\hline 706 & 52 & unk & $\mathrm{D}$ & 7.2 & 6.00 \\
\hline 706 & 53 & FRAM & $\mathrm{L}$ & 12.4 & 16.25 \\
\hline 706 & 54 & FAGR & $\mathrm{L}$ & 10.3 & 12.65 \\
\hline 706 & 55 & FRAM & $\mathrm{L}$ & 13.6 & 18.84 \\
\hline 706 & 56 & unk & D & 7.4 & 9.36 \\
\hline 706 & 57 & BELE & $\mathrm{L}$ & 14.2 & 20.23 \\
\hline 706 & 58 & BELE & $\mathrm{L}$ & 17.8 & 16.80 \\
\hline 706 & 59 & BELE & $\mathrm{L}$ & 16.6 & 20.23 \\
\hline 706 & 60 & PRSE & $\mathrm{L}$ & 28.3 & 19.84 \\
\hline 706 & 61 & BELE & $\mathrm{L}$ & 23.6 & 25.28 \\
\hline 706 & 62 & ACSA & $\mathrm{L}$ & 8.6 & 16.35 \\
\hline 706 & 63 & FAGR & $\mathrm{L}$ & 6.0 & 8.00 \\
\hline 706 & 64 & BELE & $\mathrm{L}$ & 23.0 & 20.06 \\
\hline 1301 & 1 & ACSA & $\mathrm{L}$ & 16.3 & 20.16 \\
\hline 1301 & 2 & ACSA & $\mathrm{L}$ & 51.5 & 33.93 \\
\hline 1301 & 3 & ACSA & $\mathrm{L}$ & 17.5 & 20.79 \\
\hline 1301 & 4 & ACSA & $\mathrm{L}$ & 17.7 & 23.04 \\
\hline 1301 & 5 & ACSA & $\mathrm{L}$ & 21.3 & 27.45 \\
\hline 1301 & 6 & ACSA & $\mathrm{L}$ & 12.2 & 23.45 \\
\hline 1301 & 7 & unk & D & 19.0 & 7.00 \\
\hline 1301 & 8 & ACSA & $\mathrm{L}$ & 20.1 & 21.88 \\
\hline 1301 & 9 & ACSA & $\mathrm{L}$ & 9.3 & 12.20 \\
\hline 1301 & 10 & ACSA & $\mathrm{L}$ & 23.8 & 21.83 \\
\hline 1301 & 11 & ACSA & $\mathrm{L}$ & 26.3 & 9.36 \\
\hline 1301 & 12 & ACSA & $\mathrm{L}$ & 6.7 & 26.98 \\
\hline 1301 & 13 & ACSA & $\mathrm{L}$ & 35.8 & 27.15 \\
\hline 1301 & 14 & FAGR & $\mathrm{L}$ & 36.5 & 31.27 \\
\hline 1302 & 1 & FAGR & $\mathrm{L}$ & 25.5 & 19.13 \\
\hline 1302 & 2 & FAGR & $\mathrm{L}$ & 12.8 & 11.25 \\
\hline 1302 & 3 & FAGR & $\mathrm{L}$ & 11.8 & 10.13 \\
\hline 1302 & 4 & unk & D & 14.5 & 6.36 \\
\hline 1302 & 5 & unk & D & 28.2 & 12.09 \\
\hline 1302 & 6 & FAGR & $\mathrm{L}$ & 6.1 & 6.24 \\
\hline 1302 & 7 & FAGR & $\mathrm{L}$ & 14.1 & 11.59 \\
\hline 1302 & 8 & unk & D & 17.5 & 10.00 \\
\hline
\end{tabular}

\begin{tabular}{|c|c|c|c|c|c|}
\hline plot \# & tree \# & species & status & dbh (cm) & tree height (m) \\
\hline 1302 & 9 & QUPR & $\mathrm{L}$ & 34.0 & 24.78 \\
\hline 1302 & 10 & FAGR & $\mathrm{L}$ & 9.5 & 10.11 \\
\hline 1302 & 11 & unk & D & 22.9 & 20.70 \\
\hline 1302 & 12 & unk & $\mathrm{D}$ & 15.6 & 2.50 \\
\hline 1302 & 13 & unk & $\mathrm{D}$ & 8.5 & 13.50 \\
\hline 1302 & 14 & FAGR & $\mathrm{L}$ & 5.7 & 7.02 \\
\hline 1302 & 15 & unk & $\mathrm{D}$ & 16.5 & 4.00 \\
\hline 1302 & 16 & ACSA & $\mathrm{L}$ & 31.0 & 26.25 \\
\hline 1302 & 17 & FAGR & $\mathrm{L}$ & 8.4 & 14.04 \\
\hline 1302 & 18 & FAGR & $\mathrm{L}$ & 6.7 & 9.90 \\
\hline 1302 & 19 & FAGR & $\mathrm{L}$ & 11.9 & 16.56 \\
\hline 1302 & 20 & unk & $\mathrm{D}$ & 26.0 & 26.45 \\
\hline 1302 & 21 & unk & $\mathrm{D}$ & 22.0 & 11.50 \\
\hline 1302 & 22 & FAGR & $\mathrm{L}$ & 8.7 & 12.74 \\
\hline 1302 & 23 & FAGR & $\mathrm{L}$ & 7.7 & 7.65 \\
\hline 1302 & 24 & FAGR & $\mathrm{L}$ & 17.4 & 18.29 \\
\hline 1302 & 25 & FAGR & $\mathrm{L}$ & 25.3 & 26.07 \\
\hline 1302 & 26 & FAGR & $\mathrm{L}$ & 27.1 & 16.80 \\
\hline 1302 & 27 & FAGR & $\mathrm{L}$ & 11.6 & 12.87 \\
\hline 1302 & 28 & unk & $\mathrm{D}$ & 15.5 & 5.52 \\
\hline 1302 & 29 & QURU & $\mathrm{L}$ & 77.0 & 35.71 \\
\hline 1302 & 30 & QURU & $\mathrm{L}$ & 40.5 & 29.05 \\
\hline 1302 & 31 & FAGR & L & 24.3 & 22.56 \\
\hline 1302 & 32 & FAGR & $\mathrm{L}$ & 5.9 & 7.00 \\
\hline 1303 & 1 & TIAM & $\mathrm{L}$ & 9.3 & 12.42 \\
\hline 1303 & 2 & TIAM & $\mathrm{L}$ & 19.4 & 21.87 \\
\hline 1303 & 3 & TIAM & $\mathrm{L}$ & 38.7 & 25.92 \\
\hline 1303 & 4 & TIAM & $\mathrm{L}$ & 29.1 & 25.38 \\
\hline 1303 & 5 & FAGR & $\mathrm{L}$ & 7.8 & 10.15 \\
\hline 1303 & 6 & FAGR & $\mathrm{L}$ & 15.3 & 15.96 \\
\hline 1303 & 7 & FAGR & $\mathrm{L}$ & 11.5 & 13.20 \\
\hline 1303 & 8 & MAAC & $\mathrm{L}$ & 24.4 & 24.64 \\
\hline 1303 & 9 & ACSA & $\mathrm{L}$ & 7.4 & 9.72 \\
\hline 1303 & 10 & $\mathrm{CACO}$ & $\mathrm{L}$ & 28.5 & 32.34 \\
\hline 1303 & 11 & LITU & $\mathrm{L}$ & 25.6 & 32.00 \\
\hline 1303 & 12 & $\mathrm{CACO}$ & $\mathrm{L}$ & 18.3 & 25.65 \\
\hline 1303 & 13 & $\mathrm{CACO}$ & $\mathrm{D}$ & 14.0 & 15.75 \\
\hline 1303 & 14 & ACSA & $\mathrm{L}$ & 19.6 & 25.00 \\
\hline 1303 & 15 & FAGR & $\mathrm{L}$ & 25.2 & 30.80 \\
\hline 1303 & 16 & ACSA & $\mathrm{L}$ & 7.5 & 9.60 \\
\hline 1303 & 17 & ACSA & $\mathrm{L}$ & 9.8 & 13.20 \\
\hline 1303 & 18 & ACRU & $\mathrm{L}$ & 17.6 & 14.70 \\
\hline 1303 & 19 & TIAM & $\mathrm{L}$ & 64.1 & 37.50 \\
\hline 1304 & 1 & ACSA & L & 20.9 & 17.08 \\
\hline
\end{tabular}




\begin{tabular}{|c|c|c|c|c|c|}
\hline plot \# & tree \# & species & status & dbh (cm) & tree height (m) \\
\hline 1304 & 2 & ACSA & $\mathrm{L}$ & 6.0 & 8.68 \\
\hline 1304 & 3 & FAGR & $\mathrm{L}$ & 7.0 & 6.50 \\
\hline 1304 & 4 & ACSA & $\mathrm{L}$ & 17.4 & 20.30 \\
\hline 1304 & 5 & MATR & $\mathrm{L}$ & 8.1 & 10.40 \\
\hline 1304 & 6 & ACSA & $\mathrm{L}$ & 7.3 & 9.00 \\
\hline 1304 & 7 & unk & $\mathrm{D}$ & 15.8 & 14.22 \\
\hline 1304 & 8 & ACSA & $\mathrm{L}$ & 6.2 & 8.19 \\
\hline 1304 & 9 & ACSA & $\mathrm{L}$ & 8.4 & 9.36 \\
\hline 1304 & 10 & QUCO & $\mathrm{L}$ & 71.9 & 33.54 \\
\hline 1304 & 11 & FAGR & $\mathrm{L}$ & 5.2 & 5.39 \\
\hline 1304 & 12 & ACSA & $\mathrm{L}$ & 13.2 & 18.43 \\
\hline 1304 & 13 & unk & $\mathrm{D}$ & 17.8 & 14.90 \\
\hline 1304 & 14 & ACSA & $\mathrm{L}$ & 12.7 & 12.58 \\
\hline 1304 & 15 & QURU & $\mathrm{L}$ & 46.0 & 26.28 \\
\hline 1304 & 16 & QURU & $\mathrm{L}$ & 33.7 & 27.75 \\
\hline 1304 & 17 & ACSA & $\mathrm{L}$ & 8.4 & 6.50 \\
\hline 1304 & 18 & ACSA & $\mathrm{L}$ & 19.7 & 20.77 \\
\hline 1304 & 19 & QURU & $\mathrm{L}$ & 31.2 & 25.38 \\
\hline 1304 & 20 & NYSY & $\mathrm{L}$ & 35.4 & 21.33 \\
\hline 1304 & 21 & MATR & $\mathrm{L}$ & 24.8 & 16.44 \\
\hline 1304 & 22 & QURU & $\mathrm{L}$ & 48.7 & 30.71 \\
\hline 1304 & 23 & MATR & $\mathrm{L}$ & 39.5 & 26.03 \\
\hline 1304 & 24 & ACSA & $\mathrm{L}$ & 7.5 & 10.88 \\
\hline 1304 & 25 & ACSA & $\mathrm{L}$ & 12.1 & 13.58 \\
\hline 1304 & 26 & ACSA & $\mathrm{L}$ & 15.1 & 18.96 \\
\hline 1304 & 27 & ACSA & $\mathrm{L}$ & 20.1 & 19.80 \\
\hline 1305 & 1 & ACSA & $\mathrm{L}$ & 21.3 & 18.13 \\
\hline 1305 & 2 & MAAC & $\mathrm{L}$ & 47.1 & 26.00 \\
\hline 1305 & 3 & ACSA & $\mathrm{L}$ & 5.5 & 6.50 \\
\hline 1305 & 4 & ACSA & $\mathrm{L}$ & 7.3 & 10.50 \\
\hline 1305 & 5 & ACSA & $\mathrm{L}$ & 11.5 & 13.75 \\
\hline 1305 & 6 & QURU & $\mathrm{L}$ & 26.7 & 26.35 \\
\hline 1305 & 7 & FAGR & $\mathrm{L}$ & 21.0 & 14.95 \\
\hline
\end{tabular}

\begin{tabular}{|c|c|c|c|c|c|}
\hline plot \# & tree \# & species & status & dbh (cm) & tree height (m) \\
\hline 1305 & 8 & FAGR & $\mathrm{L}$ & 38.5 & 27.30 \\
\hline 1305 & 9 & ACSA & $\mathrm{L}$ & 7.4 & 11.00 \\
\hline 1305 & 10 & ACSA & $\mathrm{D}$ & 5.7 & 6.00 \\
\hline 1305 & 11 & ACSA & $\mathrm{L}$ & 25.1 & 24.30 \\
\hline 1305 & 12 & ACSA & $\mathrm{L}$ & 11.0 & 12.15 \\
\hline 1305 & 13 & ACSA & $\mathrm{L}$ & 5.3 & 5.50 \\
\hline 1305 & 14 & unk & $\mathrm{D}$ & 27.1 & 3.00 \\
\hline 1305 & 15 & ACSA & $\mathrm{L}$ & 7.2 & 6.00 \\
\hline 1305 & 16 & ACSA & $\mathrm{L}$ & 7.3 & 8.00 \\
\hline 1305 & 17 & ACSA & $\mathrm{L}$ & 5.5 & 5.00 \\
\hline 1305 & 18 & ACSA & $\mathrm{L}$ & 22.6 & 11.90 \\
\hline 1306 & 1 & unk & $\mathrm{D}$ & 19.6 & 5.40 \\
\hline 1306 & 2 & QUAL & $\mathrm{L}$ & 38.0 & 28.38 \\
\hline 1306 & 3 & ACSA & $\mathrm{L}$ & 5.8 & 7.50 \\
\hline 1306 & 4 & unk & $\mathrm{D}$ & 19.5 & 12.00 \\
\hline 1306 & 5 & ACSA & $\mathrm{L}$ & 30.2 & 21.25 \\
\hline 1306 & 6 & ACSA & $\mathrm{L}$ & 30.5 & 20.25 \\
\hline 1306 & 7 & ACSA & $\mathrm{L}$ & 11.0 & 12.00 \\
\hline 1306 & 8 & unk & $\mathrm{D}$ & 25.0 & 21.00 \\
\hline 1306 & 9 & unk & $\mathrm{D}$ & 28.8 & 23.24 \\
\hline 1306 & 10 & QURU & $\mathrm{L}$ & 56.2 & 25.58 \\
\hline 1306 & 11 & QURU & $\mathrm{L}$ & 88.5 & 25.58 \\
\hline 1306 & 12 & ACSA & $\mathrm{L}$ & 34.2 & 23.20 \\
\hline 1306 & 13 & ACSA & $\mathrm{L}$ & 7.4 & 10.00 \\
\hline 1306 & 14 & QURU & $\mathrm{L}$ & 47.5 & 26.25 \\
\hline 1306 & 15 & FAGR & $\mathrm{L}$ & 29.6 & 28.14 \\
\hline 1306 & 16 & QURU & $\mathrm{L}$ & 54.0 & 33.06 \\
\hline 1306 & 17 & QURU & $\mathrm{L}$ & 14.2 & 11.55 \\
\hline 1306 & 18 & ACSA & $\mathrm{L}$ & 5.5 & 14.00 \\
\hline 1306 & 19 & QURU & $\mathrm{L}$ & 37.1 & 29.12 \\
\hline 1306 & 20 & QURU & $\mathrm{D}$ & 23.1 & 20.00 \\
\hline 1306 & 21 & QURU & $\mathrm{L}$ & 7.9 & 6.00 \\
\hline
\end{tabular}




\section{APPENDIX G Increment Core Measurements*}

\section{Watershed 7}

070101A $1977 \quad 834 \quad 976 \quad 498$

$\begin{array}{lllllllllll}070101 \mathrm{~A} 1980 & 288 & 464 & 818 & 634 & 442 & 464 & 472 & 188 & 150 & 170\end{array}$ $\begin{array}{llllllllllll}070101 \mathrm{~A} & 1990 & 178 & 100 & 74 & 116 & 172 & 296 & 124 & 110 & 262 & 334\end{array}$ 070101A $2000 \quad 412 \quad 250 \quad 58-9999$

070101B $1975 \quad 228 \quad 614 \quad 538 \quad 698 \quad 568$

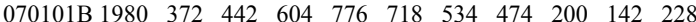
$\begin{array}{lllllllllll}070101 \mathrm{~B} 1990 & 262 & 136 & 228 & 208 & 196 & 180 & 180 & 212 & 498 & 266\end{array}$ 070101B $2000 \quad 526 \quad 392 \quad 38-9999$

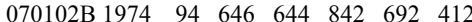

$\begin{array}{lllllllllll}070102 \mathrm{~B} 1980 & 300 & 328 & 264 & 166 & 196 & 284 & 160 & 66 & 146 & 182\end{array}$ $\begin{array}{lllllllllll}070102 \mathrm{~B} 1990 & 230 & 180 & 210 & 152 & 188 & 250 & 230 & 228 & 196 & 172\end{array}$ 070102B $2000 \quad 368 \quad 200 \quad 120-9999$

070103 A $1977 \quad 876 \quad 634 \quad 518$

$\begin{array}{lllllllllll}070103 \text { A } 1980 & 536 & 672 & 942 & 788 & 776 & 926 & 678 & 180 & 136 & 492\end{array}$

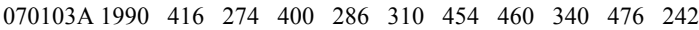
070103A $2000 \quad 492 \quad 552 \quad 142-9999$

070103B $1977 \quad 616 \quad 752 \quad 566$

$\begin{array}{lllllllllll}070103 \mathrm{~B} 1980 & 342 & 340 & 510 & 652 & 702 & 720 & 764 & 316 & 176 & 390\end{array}$

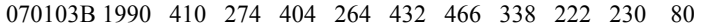
070103B $2000 \quad 196 \quad 452 \quad 106-9999$

070104A $1975 \quad 296 \quad 738 \quad 1342 \quad 466 \quad 262$

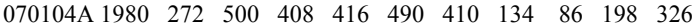

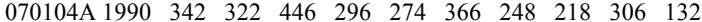
070104A $2000 \quad 134 \quad 110 \quad 50-9999$

070104B $1977 \quad 168 \quad 1120 \quad 536$

070104B 1980 $204 \begin{array}{lllllllll}220 & 378 & 460 & 302 & 574 & 574 & 140 & 136 & 304\end{array}$

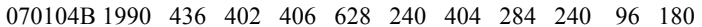
070104B $2000 \quad 94 \quad 318 \quad 198-9999$

070105A 1989240

$\begin{array}{lllllllllll}070105 \mathrm{~A} 1990 & 432 & 400 & 430 & 302 & 300 & 270 & 146 & 114 & 88 & 128\end{array}$ 070105A $2000 \quad 82 \quad 116 \quad 94-9999$

070105B $1988 \quad 210 \quad 378$

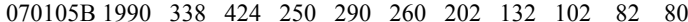
070105B $2000 \quad 34 \quad 72 \quad 102-9999$

070106A $1978 \quad 68 \quad 548$

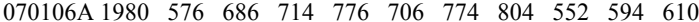

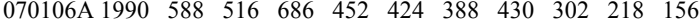
070106A $2000 \quad 334 \quad 382 \quad 104-9999$

070106B 19791362

070106B 1980 $596 \begin{array}{llllllllll}590 & 832 & 898 & 764 & 700 & 674 & 680 & 462 & 478\end{array}$

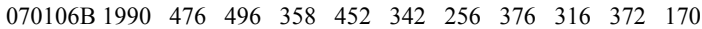
070106B $2000 \quad 278 \quad 274 \quad 72-9999$

070107A $1975 \quad 60 \quad 214 \quad 576 \quad 570 \quad 714$

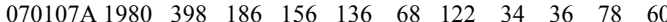

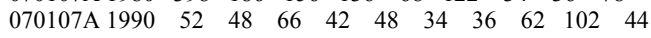

070107A $2000 \quad 44 \quad 108 \quad 24-9999$

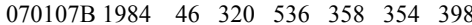

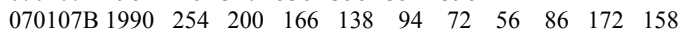

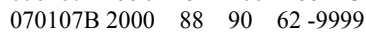

070108A 1979466

$\begin{array}{lllllllllll}070108 \text { A } 1980 & 376 & 360 & 248 & 244 & 188 & 118 & 236 & 190 & 246 & 146\end{array}$

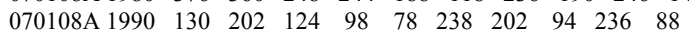
070108A $2000 \quad 134 \quad 128 \quad 40-9999$

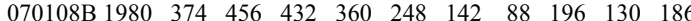

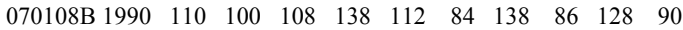
070108B $2000 \quad 148 \quad 72 \quad 230-9999$

070109A $1984 \quad 284 \quad 364 \quad 522 \quad 296 \quad 344 \quad 410$

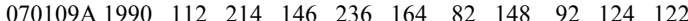
070109A $2000 \quad 106 \quad 74 \quad 132-9999$

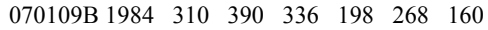

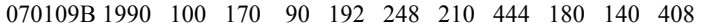

070109B $2000 \quad 90 \quad 206 \quad 256-9999$

070110A $1975 \quad 554 \quad 660 \quad 580 \quad 504 \quad 268$

$\begin{array}{lllllllllll}070110 \text { A } 1980 & 252 & 162 & 184 & 182 & 166 & 224 & 180 & 118 & 100 & 134\end{array}$

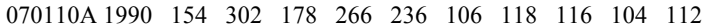
070110A $2000 \quad 162 \quad 150 \quad 46-9999$

070111A $1974 \quad 262 \quad 734 \quad 908 \quad 986 \quad 1258 \quad 894$

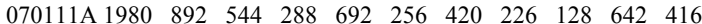
$\begin{array}{lllllllllll}070111 \mathrm{~A} 1990 & 426 & 468 & 782 & 550 & 772 & 616 & 728 & 474 & 514 & 346\end{array}$ 070111A $2000 \quad 310 \quad 364 \quad 214-9999$
070111B $19742546 \quad 1112 \quad 560 \quad 372 \quad 772 \quad 684$

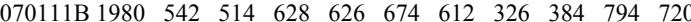
$\begin{array}{lllllllllll}070111 \mathrm{~B} 1990 & 482 & 592 & 692 & 504 & 280 & 628 & 758 & 612 & 574 & 354\end{array}$ 070111B $2000 \quad 702 \quad 228 \quad 114-9999$

070112 A $1977 \quad 82 \quad 424 \quad 370$

$\begin{array}{lllllllllll}070112 \text { A } 1980 & 268 & 128 & 132 & 100 & 86 & 148 & 124 & 158 & 162 & 156\end{array}$ $\begin{array}{lllllllllll}070112 \mathrm{~A} 1990 & 158 & 76 & 82 & 46 & 58 & 76 & 48 & 84 & 88 & 152\end{array}$ 070112A $2000 \quad 64 \quad 60 \quad 58-9999$

$070112 \mathrm{~B} 1979318$

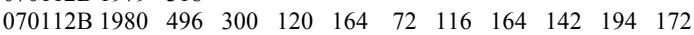

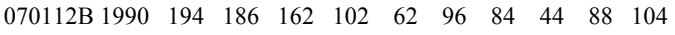
$\begin{array}{llllll}070112 B & 2000 & 84 & 56 & 140 & -9999\end{array}$

070113A $1974 \quad 112 \quad 654 \quad 742 \quad 736 \quad 884 \quad 658$

$\begin{array}{lllllllllll}070113 \text { A } 1980 & 828 & 764 & 452 & 556 & 376 & 298 & 192 & 112 & 78 & 82\end{array}$

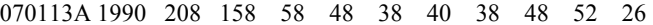

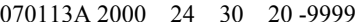

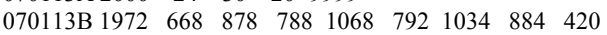

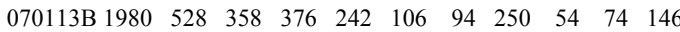

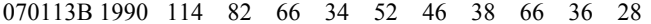
070113B $2000 \quad 50 \quad 40 \quad 42-9999$

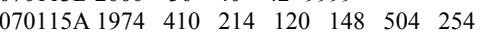

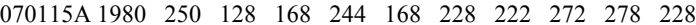

$\begin{array}{llllllllllll}070115 \mathrm{~A} & 1990 & 204 & 146 & 124 & 86 & 56 & 112 & 58 & 34 & 76 & 94\end{array}$ 070115A $2000 \quad 92 \quad 112 \quad 62-9999$

070115B 1979238

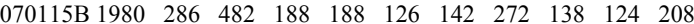

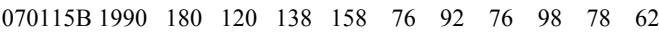
070115B $2000 \quad 76 \quad 56 \quad 156-9999$

070116A $1974 \quad 912 \quad 1032 \quad 906 \quad 602 \quad 1052 \quad 702$

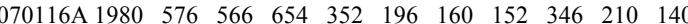
$\begin{array}{llllllllllll}\text { 070116A } 1990 & 136 & 174 & 164 & 160 & 156 & 150 & 154 & 400 & 84 & 128\end{array}$ 070116A $2000 \quad 180 \quad 110 \quad 70-9999$

$\begin{array}{lllllllllll}070116 \mathrm{~B} & 1971 & 364 & 732 & 828 & 884 & 734 & 346 & 418 & 468 & 378\end{array}$

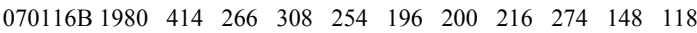

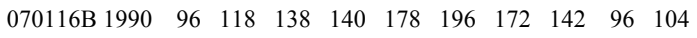
070116B 2000 $196 \quad 118 \quad 74-9999$

070117A $1985 \quad 336 \quad 734 \quad 782 \quad 730 \quad 800$

$\begin{array}{lllllllllll}\text { 070117A } 1990 & 408 & 428 & 406 & 184 & 184 & 202 & 154 & 78 & 72 & 58\end{array}$ 070117A $2000 \quad 72 \quad 50 \quad 64-9999$

070117B $1987 \quad 332 \quad 734 \quad 718$

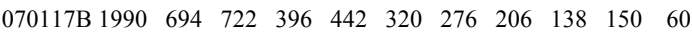
070117B $2000 \quad 56 \quad 66 \quad 70-9999$

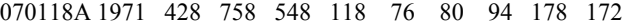

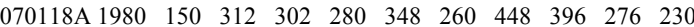

$\begin{array}{lllllllllll}070118 \text { A } 1990 & 184 & 208 & 158 & 194 & 158 & 108 & 94 & 84 & 60 & 96\end{array}$

070118A $2000 \quad 84 \quad 130 \quad 46-9999$

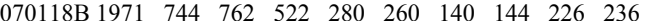

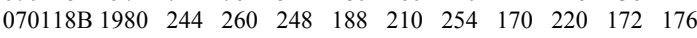

$\begin{array}{lllllllllll}070118 \mathrm{~B} 1990 & 194 & 138 & 240 & 236 & 178 & 124 & 112 & 72 & 82 & 128\end{array}$

070118B $2000 \quad 160 \quad 172 \quad 26-9999$

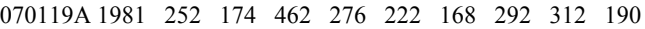

$\begin{array}{lllllllllll}070119 \text { A } 1990 & 206 & 116 & 114 & 82 & 130 & 92 & 48 & 42 & 48 & 38\end{array}$

070119A $2000 \quad 70 \quad 78 \quad 92-9999$

070119B $1977 \quad 102 \quad 112 \quad 126$

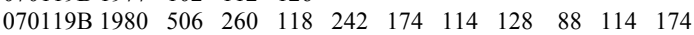

070119B 1990 $164 \begin{array}{llllllllll}186 & 112 & 110 & 106 & 92 & 64 & 46 & 96 & 178\end{array}$

070119B 2000 $158 \quad 70 \quad 68-9999$

070120A $1979 \quad 36$

$\begin{array}{lllllllllll}070120 \text { A } 1980 & 592 & 368 & 512 & 196 & 146 & 146 & 210 & 170 & 162 & 200\end{array}$ $\begin{array}{lllllllllll}070120 \text { A } 1990 & 94 & 110 & 122 & 188 & 120 & 120 & 192 & 68 & 162 & 92\end{array}$

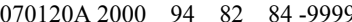

070120B 1970 $298 \begin{array}{llllllllll}508 & 336 & 220 & 234 & 92 & 68 & 118 & 94 & 142\end{array}$ $\begin{array}{lllllllllll}070120 \mathrm{~B} 1980 & 182 & 154 & 248 & 90 & 112 & 106 & 96 & 70 & 80 & 110\end{array}$

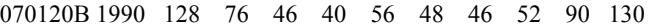
070120B $2000 \quad 68 \quad 184 \quad 72-9999$

070121A $1973 \quad 846 \quad 646 \quad 940 \quad 416 \quad 560 \quad 534 \quad 218$

$\begin{array}{llllllllllll}070121 \mathrm{~A} & 1980 & 300 & 226 & 236 & 158 & 100 & 122 & 182 & 204 & 162 & 156\end{array}$

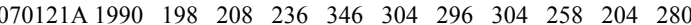
$\begin{array}{lllll}070121 \text { A } 2000 & 244 & 194 & 106 & -9999\end{array}$

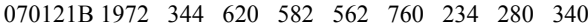

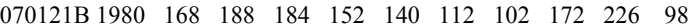




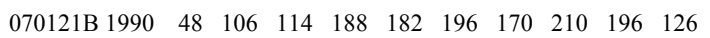
070121B $2000 \quad 124 \quad 106 \quad 172-9999$

$\begin{array}{llllll}070122 \mathrm{~A} & 1976 & 238 & 316 & 230 & 140\end{array}$

$\begin{array}{lllllllllll}070122 \mathrm{~A} 1980 & 218 & 158 & 218 & 316 & 252 & 336 & 326 & 348 & 444 & 280\end{array}$ $\begin{array}{lllllllllll}070122 \mathrm{~A} 1990 & 282 & 178 & 240 & 222 & 334 & 78 & 38 & 130 & 196 & 102\end{array}$ $\begin{array}{llllll}070122 \mathrm{~A} & 2000 & 112 & 158 & 60 & -9999\end{array}$

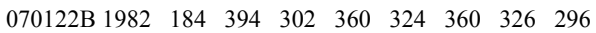

$\begin{array}{lllllllllll}070122 \mathrm{~B} 1990 & 378 & 240 & 204 & 180 & 156 & 166 & 236 & 214 & 196 & 138\end{array}$

070122B $2000 \quad 98 \quad 148 \quad 84-9999$

070123A $1978 \quad 456 \quad 400$

$\begin{array}{lllllllllll}070123 \text { A } 1980 & 240 & 120 & 156 & 122 & 86 & 150 & 262 & 240 & 230 & 248\end{array}$

$\begin{array}{lllllllllll}070123 \mathrm{~A} 1990 & 244 & 374 & 318 & 252 & 174 & 226 & 424 & 202 & 146 & 386\end{array}$ $\begin{array}{llllll}070123 \text { A } 2000 & 134 & 178 & 66 & -9999\end{array}$

070123B $1976 \quad 280 \quad 276 \quad 160 \quad 148$

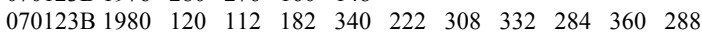

$\begin{array}{llllllllllll}070123 \mathrm{~B} 1990 & 230 & 168 & 182 & 198 & 184 & 198 & 176 & 98 & 100 & 194\end{array}$ 070123B 2000 $162 \quad 178 \quad 84-9999$

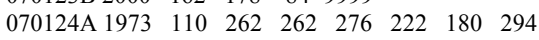

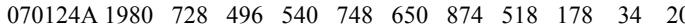

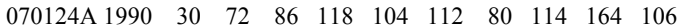

070124A $2000 \quad 140 \quad 130 \quad 34-9999$

070124B 197970

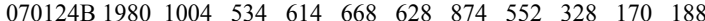

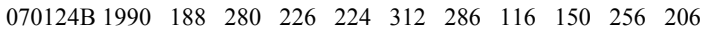
070124B $2000 \quad 212 \quad 232 \quad 66-9999$

$070125 \mathrm{~A} 1975 \quad 262 \quad 526 \quad 424 \quad 526 \quad 330$

$\begin{array}{lllllllllll}070125 \text { A } 1980 & 304 & 418 & 162 & 236 & 274 & 290 & 272 & 100 & 268 & 276\end{array}$ $\begin{array}{llllllllllll}070125 \mathrm{~A} & 1990 & 198 & 102 & 180 & 202 & 150 & 266 & 278 & 294 & 336 & 88\end{array}$ 070125A $2000 \quad 46 \quad 164 \quad 46-9999$

070125B $1976 \quad 556 \quad 326 \quad 416 \quad 368$

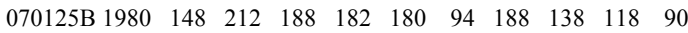

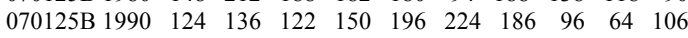

070125B $2000 \quad 130 \quad 40 \quad 32-9999$

070127A $1978 \quad 238 \quad 224$

$\begin{array}{lllllllllll}\text { 070127A } 1980 & 178 & 146 & 84 & 114 & 154 & 176 & 192 & 222 & 188 & 234\end{array}$ $\begin{array}{lllllllllll}070127 \mathrm{~A} 1990 & 172 & 118 & 276 & 140 & 80 & 140 & 164 & 62 & 144 & 132\end{array}$ 070127A $2000 \quad 158 \quad 178 \quad 28-9999$

070127B $1977 \quad 708 \quad 254 \quad 370$

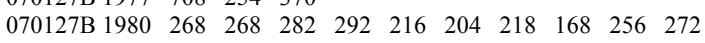
$\begin{array}{lllllllllll}070127 \mathrm{~B} 1990 & 158 & 122 & 184 & 182 & 226 & 196 & 156 & 112 & 200 & 132\end{array}$ 070127B $2000 \quad 164 \quad 140 \quad 102-9999$

070129A $1978 \quad 246 \quad 454$

070129A 1980 $396 \begin{array}{llllllllll}460 & 280 & 364 & 266 & 200 & 368 & 274 & 252 & 264\end{array}$

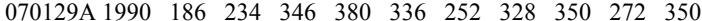
070129A $2000 \quad 286 \quad 428 \quad 348-9999$

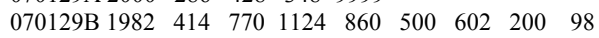

070129B 1990 $176 \begin{array}{lllllllll}168 & 152 & 134 & 198 & 196 & 136 & 158 & 152 & 212\end{array}$ 070129B $2000 \quad 130 \quad 88 \quad 108-9999$

$\begin{array}{llllllll}070130 \text { A } 1983 & 276 & 314 & 364 & 270 & 310 & 330 & 268\end{array}$

$\begin{array}{lllllllllll}070130 \text { A } 1990 & 116 & 114 & 126 & 148 & 88 & 90 & 60 & 58 & 50 & 58\end{array}$

070130A $2000 \quad 56 \quad 50 \quad 98-9999$

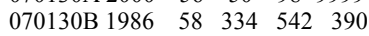

$\begin{array}{lllllllllll}070130 \mathrm{~B} 1990 & 422 & 272 & 160 & 152 & 110 & 226 & 280 & 146 & 74 & 50\end{array}$

070130B 2000 $62 \quad 54 \quad 110-9999$

070131A 1979342

$\begin{array}{lllllllllll}070131 \mathrm{~A} 1980 & 284 & 256 & 304 & 182 & 188 & 206 & 364 & 346 & 284 & 378\end{array}$

$\begin{array}{lllllllllll}070131 \mathrm{~A} 1990 & 196 & 210 & 176 & 118 & 124 & 102 & 122 & 164 & 206 & 376\end{array}$

$\begin{array}{lllll}070131 \text { A } 2000 & 314 & 240 & 276 & -9999\end{array}$

070131B $1976 \quad 332 \quad 524 \quad 458 \quad 368$

$\begin{array}{lllllllllll}070131 \mathrm{~B} 1980 & 338 & 186 & 206 & 178 & 206 & 216 & 278 & 328 & 184 & 182\end{array}$

$\begin{array}{lllllllllll}\text { 070131B } 1990 & 140 & 76 & 104 & 88 & 100 & 124 & 118 & 68 & 74 & 58\end{array}$

070131B 2000 $94 \quad 82 \quad 70-9999$

$\begin{array}{llllllll}070132 \text { A } 1973 & 202 & 142 & 178 & 174 & 300 & 278 & 86\end{array}$

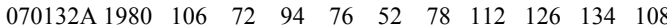

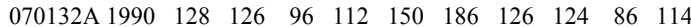

$\begin{array}{lllll}070132 \text { A } 2000 & 142 & 108 & 96 & -9999\end{array}$

070132B $1975 \quad 122 \quad 804 \quad 446 \quad 410 \quad 332$

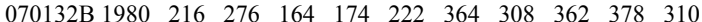

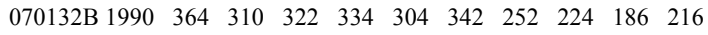

070132B $2000 \quad 282 \quad 244 \quad 84-9999$

$\begin{array}{lllllllll}070134 \mathrm{~A} & 1983 & 82 & 556 & 276 & 152 & 126 & 136 & 188\end{array}$

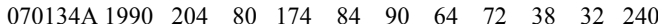

070134A $2000 \quad 74 \quad 76 \quad 38-9999$

070134B 1979352

$\begin{array}{lllllllllll}070134 \mathrm{~B} 1980 & 576 & 424 & 312 & 210 & 146 & 108 & 230 & 172 & 272 & 230\end{array}$

$\begin{array}{lllllllllll}070134 \mathrm{~B} 1990 & 216 & 172 & 150 & 148 & 106 & 134 & 38 & 64 & 160 & 168\end{array}$

070134B 2000 $208 \quad 116 \quad 40-9999$

$\begin{array}{lllllll}070135 \mathrm{~A} 1974 & 132 & 430 & 198 & 248 & 256 & 158\end{array}$

$\begin{array}{llllllllllll}070135 \mathrm{~A} & 1980 & 314 & 340 & 334 & 420 & 356 & 458 & 402 & 296 & 132 & 228\end{array}$

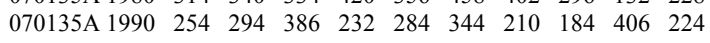

070135A $2000 \quad 364 \quad 474 \quad 124-9999$

070535B $1977 \quad 632 \quad 618 \quad 548$

$\begin{array}{lllllllllll}070535 \mathrm{~B} 1980 & 690 & 590 & 586 & 636 & 764 & 916 & 604 & 902 & 876 & 648\end{array}$

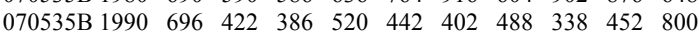
070535B $2000 \quad 1064 \quad 584 \quad 162-9999$

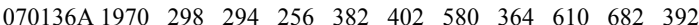
$\begin{array}{lllllllllll}070136 \text { A } 1980 & 328 & 298 & 384 & 274 & 148 & 94 & 278 & 274 & 318 & 270\end{array}$ $\begin{array}{lllllllllll}\text { 070136A } 1990 & 366 & 298 & 210 & 276 & 248 & 302 & 304 & 334 & 244 & 234\end{array}$ 070136A $2000 \quad 368 \quad 366 \quad 104-9999$

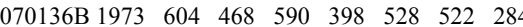

$\begin{array}{llllllllllll}070136 \mathrm{~B} & 1980 & 256 & 158 & 238 & 138 & 96 & 100 & 240 & 228 & 204 & 314\end{array}$

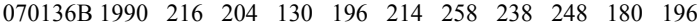
070136B $2000 \quad 318 \quad 274 \quad 94-9999$

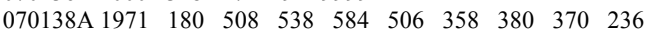

$\begin{array}{llllllllllll}\text { 070138A } 1980 & 176 & 208 & 204 & 132 & 102 & 74 & 140 & 148 & 72 & 80\end{array}$

$\begin{array}{lllllllllll}070138 \text { A } 1990 & 68 & 80 & 68 & 58 & 82 & 54 & 44 & 36 & 36 & 42\end{array}$

070138A $2000 \quad 34 \quad 36 \quad 36-9999$

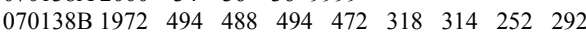

$\begin{array}{llllllllllll}070138 \mathrm{~B} & 1980 & 314 & 246 & 270 & 212 & 190 & 160 & 242 & 186 & 148 & 108\end{array}$

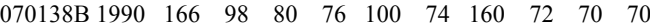

070138B $2000 \quad 40 \quad 56 \quad 40-9999$

070139A 1991 $124 \quad 404 \quad 404 \quad 400 \quad 526 \quad 424 \quad 416 \quad 348 \quad 214$

070139A 2000 $204 \quad 134 \quad 134-9999$

070139B 1989148

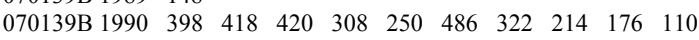
070139B $2000 \quad 144 \quad 48 \quad 98-9999$

070140A $1975 \quad 314 \quad 294 \quad 152 \quad 140 \quad 136$

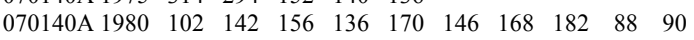

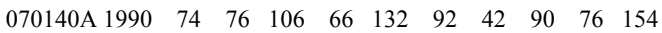

070140A $2000 \quad 112 \quad 160 \quad 160-9999$

070140B $1978 \quad 118 \quad 300$

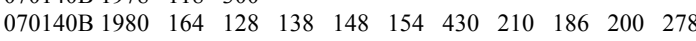

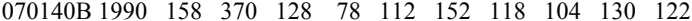
070140B $2000 \quad 222 \quad 158 \quad 46-9999$

070141A $1984 \quad 248 \quad 652 \quad 516 \quad 346 \quad 284 \quad 228$

070141A $1990 \begin{array}{lllllllllll}160 & 158 & 188 & 270 & 174 & 394 & 150 & 24 & 38 & 46\end{array}$

070141A $2000 \quad 30 \quad 30 \quad 28-9999$

070141B $1984 \quad 246 \quad 646 \quad 502 \quad 318 \quad 216 \quad 200$

$\begin{array}{llllllllllll}070141 \mathrm{~B} 1990 & 120 & 198 & 186 & 270 & 158 & 188 & 288 & 214 & 46 & 38\end{array}$

070141B 2000 $54 \quad 38 \quad 48-9999$

$\begin{array}{llllllll}070142 \mathrm{~A} & 1974 & 288 & 538 & 464 & 164 & 114 & 120\end{array}$

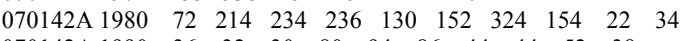

$\begin{array}{lllllllllll}070142 \mathrm{~A} 1990 & 36 & 32 & 30 & 80 & 94 & 86 & 44 & 44 & 52 & 38\end{array}$

070142A $2000 \quad 30 \quad 58 \quad 34-9999$

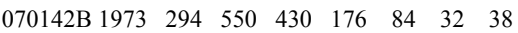

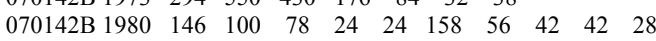

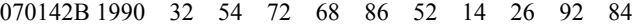

070142B 2000 $\quad 60 \quad 34 \quad 22-9999$

070143A $1985 \quad 244 \quad 496 \quad 366 \quad 356 \quad 184$

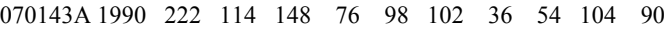

070143A $2000 \quad 172 \quad 70 \quad 94-9999$

070143B $1988 \quad 236 \quad 482$

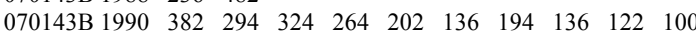

070143B 2000 $102 \quad 166 \quad 140-9999$

070144A $1976 \quad 314 \quad 476 \quad 516 \quad 450$

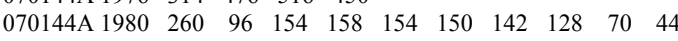

$\begin{array}{lllllllllll}070144 \mathrm{~A} 1990 & 48 & 78 & 60 & 96 & 118 & 146 & 108 & 210 & 126 & 82\end{array}$

070144A $2000 \quad 140 \quad 104 \quad 50-9999$

070144B $1975 \quad 224 \quad 500 \quad 466 \quad 302 \quad 230$

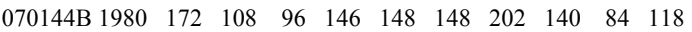

$\begin{array}{llllllllllll}070144 \mathrm{~B} & 1990 & 118 & 140 & 122 & 158 & 122 & 86 & 94 & 172 & 242 & 220\end{array}$

070144B 2000 $294 \quad 174 \quad 68-9999$

070145A $1985 \quad 314 \quad 372 \quad 432 \quad 246 \quad 370$

070145A 1990 $126 \begin{array}{llllllllll}154 & 104 & 102 & 70 & 42 & 70 & 70 & 70 & 52\end{array}$

070145A $2000 \quad 78 \quad 60 \quad 72-9999$

070145B 1989498

$\begin{array}{lllllllllll}070145 \mathrm{~B} 1990 & 366 & 274 & 222 & 258 & 48 & 148 & 84 & 84 & 66 & 82\end{array}$

070145B $2000 \quad 212 \quad 36 \quad 74-9999$

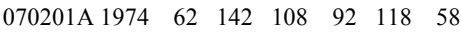

$\begin{array}{llllllllllll}070201 \mathrm{~A} & 1980 & 78 & 40 & 114 & 108 & 58 & 80 & 110 & 156 & 136 & 62\end{array}$

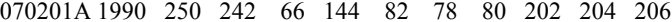

070201A $2000 \quad 164 \quad 120 \quad 106-9999$

070201B $1977 \quad 70 \quad 154 \quad 102$

$\begin{array}{lllllllllll}070201 \mathrm{~B} 1980 & 100 & 112 & 52 & 52 & 66 & 156 & 78 & 102 & 80 & 154\end{array}$

$\begin{array}{llllllllllll}070201 B & 1990 & 152 & 372 & 368 & 144 & 234 & 294 & 318 & 196 & 196 & 172\end{array}$

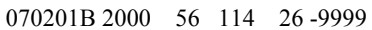

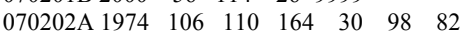

$\begin{array}{llllllllllll}070202 \mathrm{~A} & 1980 & 86 & 104 & 206 & 182 & 230 & 208 & 144 & 208 & 154 & 130\end{array}$

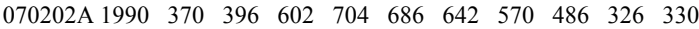

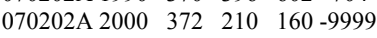


070202B $1987 \quad 166 \quad 120 \quad 152$

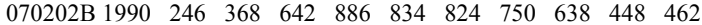
070202B $2000 \quad 414 \quad 310 \quad 200-9999$

070203A $1996 \quad 326 \quad 606 \quad 1120 \quad 660$

070203A $2000 \quad 636 \quad 370 \quad 250-9999$

070203B $1996 \quad 434 \quad 588 \quad 652 \quad 710$

070203B $2000 \quad 308 \quad 182 \quad 40-9999$

070204B 1982 $54 \quad \begin{array}{llllllll} & 52 & 62 & 64 & 64 & 64 & 48 & 46\end{array}$

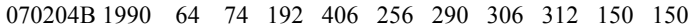

070204B $2000 \quad 138 \quad 150 \quad 76-9999$

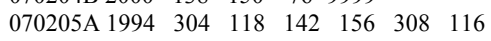

070205A $2000 \quad 534 \quad 326 \quad 68-9999$

070205B $1995 \quad 374 \quad 624 \quad 642 \quad 1086 \quad 440$

070205B $2000 \quad 602 \quad 184 \quad 68-9999$

070206A $1994 \quad 412 \quad 522 \quad 328 \quad 96 \quad 336 \quad 372$

070206A $2000 \quad 208 \quad 180 \quad 186-9999$

070206B $1993 \quad 76 \quad 508 \quad 430 \quad 248 \quad 74 \quad 236 \quad 248$

070206B $2000 \quad 84 \quad 92 \quad 64-9999$

070207A $1984 \quad 52 \quad 276 \quad 232 \quad 166 \quad 162 \quad 128$

070207A 1990 $310 \quad 188 \quad 70 \quad 72 \quad 96 \quad 178 \quad 326 \quad 356 \quad 250 \quad 292$

070207A $2000 \quad 164 \quad 140 \quad 106-9999$

070208A $1987 \quad 128 \quad 230 \quad 288$

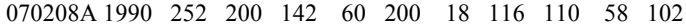
070208A $2000 \quad 110 \quad 72 \quad 80-9999$

070209A $1975 \quad 202 \quad 272 \quad 132 \quad 140 \quad 248$

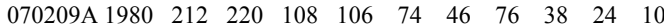

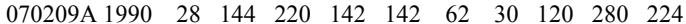

$\begin{array}{lllll}070209 \text { A } 2000 & 82 & 128 & 130 & -9999\end{array}$

070210A $1974 \quad 296 \quad 168 \quad 200 \quad 246 \quad 246 \quad 210$

070210A 1980 $240 \begin{array}{llllllllll}302 & 234 & 274 & 238 & 284 & 238 & 228 & 206 & 204\end{array}$

070210A $1990 \begin{array}{lllllllllll}110 & 80 & 58 & 68 & 32 & 34 & 32 & 46 & 50 & 54\end{array}$

070210A $2000 \quad 42 \quad 42 \quad 38-9999$

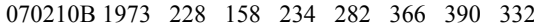

070210B 1980 $422 \begin{array}{lllllllll}360 & 526 & 440 & 468 & 466 & 400 & 442 & 380 & 304\end{array}$

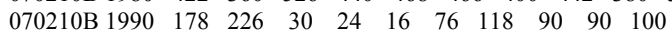

070210B $2000 \quad 82 \quad 54 \quad 48-9999$

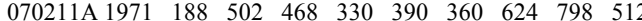

$\begin{array}{llllllllllll}070211 \mathrm{~A} & 1980 & 778 & 572 & 620 & 528 & 516 & 550 & 754 & 658 & 394 & 400\end{array}$

$\begin{array}{llllllllllll}070211 \text { A } 1990 & 310 & 264 & 294 & 476 & 606 & 746 & 614 & 588 & 698 & 572\end{array}$

070211A $2000 \quad 620 \quad 604 \quad 458-9999$

070211B $1977 \quad 50 \quad 1256 \quad 468$

$\begin{array}{lllllllllll}070211 \mathrm{~B} 1980 & 450 & 334 & 426 & 310 & 252 & 380 & 488 & 350 & 216 & 200\end{array}$

$\begin{array}{llllllllllll}070211 \mathrm{~B} & 1990 & 212 & 100 & 116 & 434 & 596 & 686 & 558 & 512 & 698 & 564\end{array}$

070211B $2000 \quad 614 \quad 576 \quad 434-9999$

$\begin{array}{llllllllll}070213 \text { A } 1981 & 292 & 534 & 262 & 102 & 84 & 142 & 140 & 142 & 196\end{array}$

$\begin{array}{lllllllllll}070213 \text { A } 1990 & 222 & 242 & 380 & 546 & 524 & 326 & 158 & 110 & 312 & 346\end{array}$

070213A $2000 \quad 242 \quad 438 \quad 428-9999$

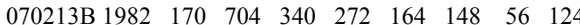

$\begin{array}{lllllllllll}070213 \mathrm{~B} 1990 & 142 & 146 & 228 & 332 & 370 & 328 & 148 & 178 & 328 & 286\end{array}$

070213B $2000306 \quad 294 \quad 300-9999$

$\begin{array}{llllllll}070215 \mathrm{~A} & 1984 & 216 & 34 & 144 & 132 & 166 & 36\end{array}$

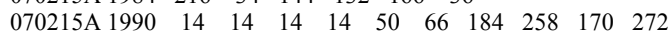

070215A $2000 \quad 124 \quad 108 \quad 104-9999$

070215B $1984 \quad 214 \quad 186 \quad 130 \quad 46 \quad 374 \quad 100$

$\begin{array}{lllllllllll}070215 \mathrm{~B} 1990 & 50 & 112 & 52 & 76 & 114 & 248 & 402 & 22 & 84 & 72\end{array}$

070215B $2000 \quad 46 \quad 76 \quad 88-9999$

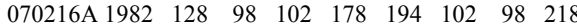

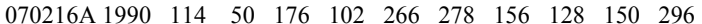

$\begin{array}{lllll}070216 \text { A } 2000 & 128 & 128 & 108 & -9999\end{array}$

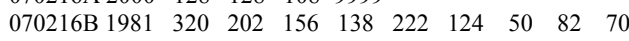

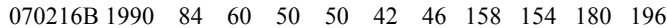

070216B $2000 \quad 192 \quad 132 \quad 208-9999$

070217A $1986 \quad 264 \quad 252 \quad 202 \quad 144$

$\begin{array}{llllllllllll}070217 \mathrm{~A} 1990 & 178 & 280 & 212 & 182 & 302 & 166 & 256 & 156 & 186 & 118\end{array}$

070217A $2000 \quad 58 \quad 88 \quad 64-9999$

$070217 \mathrm{~B} 1989 \quad 180$

$\begin{array}{lllllllllll}070217 \mathrm{~B} 1990 & 284 & 206 & 300 & 438 & 106 & 116 & 152 & 162 & 108 & 144\end{array}$

$\begin{array}{llllll}070217 \mathrm{~B} & 2000 & 174 & 54 & 80 & -9999\end{array}$

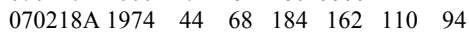

$\begin{array}{lllllllllll}070218 \text { A } 1980 & 70 & 154 & 190 & 54 & 54 & 56 & 100 & 44 & 80 & 56\end{array}$

$\begin{array}{llllllllllll}070218 \mathrm{~A} & 1990 & 72 & 104 & 296 & 230 & 164 & 308 & 248 & 342 & 386 & 340\end{array}$

070218A $2000 \quad 386 \quad 202 \quad 98-9999$

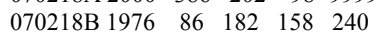

$\begin{array}{lllllllllll}070218 \mathrm{~B} 1980 & 68 & 252 & 144 & 72 & 90 & 78 & 94 & 70 & 80 & 48\end{array}$

$\begin{array}{llllllllllll}070218 B & 1990 & 70 & 74 & 316 & 244 & 164 & 220 & 156 & 202 & 206 & 200\end{array}$

070218B $2000 \quad 254 \quad 198 \quad 90-9999$

070219A $1975 \quad 152 \quad 388 \quad 208 \quad 224 \quad 194$

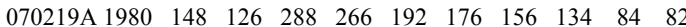

$\begin{array}{llllllllllll}070219 A & 1990 & 78 & 94 & 122 & 220 & 80 & 238 & 232 & 162 & 304 & 226\end{array}$

070219A $2000 \quad 274 \quad 234 \quad 138-9999$

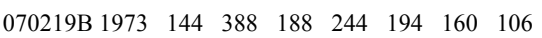

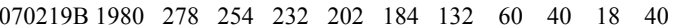

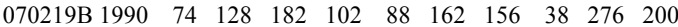

070219B 2000 $194 \quad 290 \quad 132-9999$

070220A $1978 \quad 228 \quad 184$

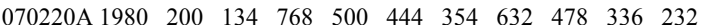

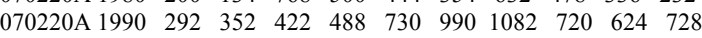

070220A $2000 \quad 892 \quad 528 \quad 234-9999$

070220B 1981 $234 \quad 390 \quad 412 \quad 468 \quad 754 \quad 770 \quad 556 \quad 326 \quad 312$

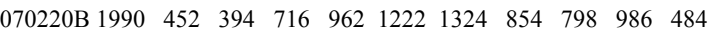

070220B $2000 \quad 786 \quad 916 \quad 362-9999$

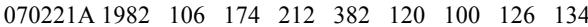

$\begin{array}{lllllllllll}070221 \mathrm{~A} 1990 & 172 & 74 & 146 & 74 & 50 & 138 & 154 & 180 & 116 & 114\end{array}$

070221A $2000 \quad 64 \quad 36 \quad 52-9999$

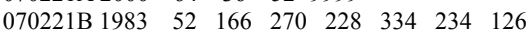

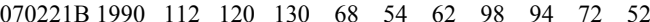

$070221 B 2000 \quad 70 \quad 46 \quad 52-9999$

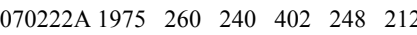

$\begin{array}{lllllllllll}070222 \text { A } 1980 & 164 & 128 & 184 & 214 & 388 & 330 & 378 & 342 & 398 & 408\end{array}$

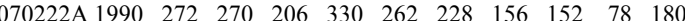

070222A $2000 \quad 172 \quad 50 \quad 36-9999$

070222B $1975 \quad 198 \quad 196 \quad 186 \quad 288 \quad 360$

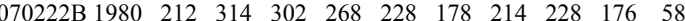

$\begin{array}{llllllllllll}070222 \mathrm{~B} 1990 & 70 & 92 & 74 & 232 & 190 & 178 & 118 & 108 & 126 & 186\end{array}$

070222B $2000 \quad 160 \quad 38 \quad 46-9999$

070223A $1977 \quad 352 \quad 556 \quad 668$

$\begin{array}{lllllllllll}070223 \text { A } 1980 & 446 & 504 & 422 & 500 & 502 & 430 & 218 & 308 & 346 & 168\end{array}$

$\begin{array}{lllllllllll}070223 \mathrm{~A} 1990 & 192 & 108 & 172 & 316 & 294 & 456 & 430 & 366 & 336 & 302\end{array}$ 070223A $2000 \quad 362 \quad 158 \quad 138-9999$

070223B $1973 \quad 406 \quad 294 \quad 366 \quad 500 \quad 312 \quad 346 \quad 430$

$\begin{array}{llllllllllll}070223 \mathrm{~B} & 1980 & 372 & 566 & 514 & 542 & 392 & 264 & 92 & 70 & 134 & 138\end{array}$

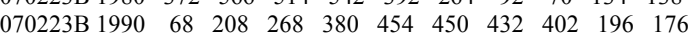

070223B $2000 \quad 136 \quad 192 \quad 158-9999$

$\begin{array}{llllll}070224 \mathrm{~A} & 1976 & 234 & 290 & 476 & 494\end{array}$

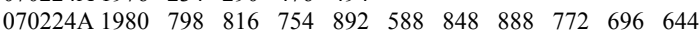

$\begin{array}{llllllllllll}070224 \mathrm{~A} & 1990 & 586 & 448 & 614 & 536 & 662 & 834 & 706 & 508 & 628 & 348\end{array}$

070224A $2000 \quad 566 \quad 598 \quad 258-9999$

070224B $1976 \quad 262 \quad 636 \quad 782 \quad 500$

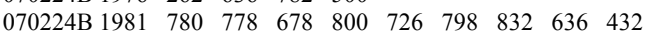

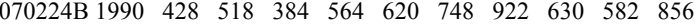

070224B $2000 \quad 970 \quad 670 \quad 302-9999$

070301A 1979302

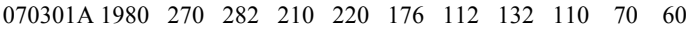

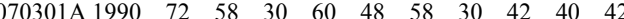

070301A $2000 \quad 80 \quad 44 \quad 66-9999$

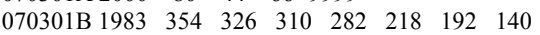

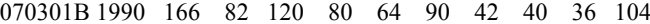

070301B $2000 \quad 92 \quad 42 \quad 42 \quad-9999$

$\begin{array}{lllllllllll}070302 \mathrm{~A} 1980 & 528 & 450 & 608 & 480 & 216 & 336 & 392 & 378 & 212 & 320\end{array}$

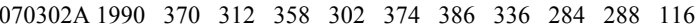

070302A $2000 \quad 250 \quad 362 \quad 108-9999$

$070302 \mathrm{~B} 1979370$

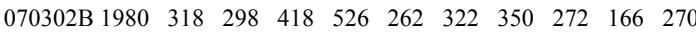

$\begin{array}{llllllllllll}070302 B & 1990 & 352 & 330 & 432 & 326 & 382 & 424 & 286 & 200 & 224 & 118\end{array}$

070302B $2000 \quad 154 \quad 280 \quad 86-9999$

070303A $1975 \quad 60 \quad 116 \quad 168 \quad 128 \quad 96$

$\begin{array}{lllllllllll}070303 \text { A } 1980 & 88 & 94 & 118 & 172 & 166 & 144 & 126 & 88 & 26 & 82\end{array}$

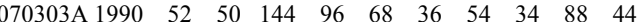

070303A $2000 \quad 58 \quad 26 \quad 44-9999$

070303B $1978 \quad 86 \quad 184$

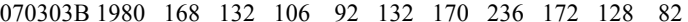

070303B $1990 \quad 50 \quad 74 \quad 52 \quad 42 \quad 148 \quad 64 \quad 64 \quad 32 \quad 50 \quad 58$

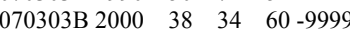

070304A $1984 \quad 44 \quad 280 \quad 246 \quad 136 \quad 98 \quad 222$

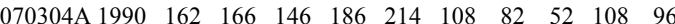

070304A $2000 \quad 82 \quad 62 \quad 54-9999$

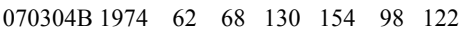

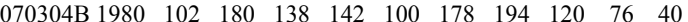

070304B 1990 $60 \begin{array}{llllllllll}46 & 28 & 80 & 42 & 24 & 14 & 16 & 12 & 18\end{array}$

070304B 2000 $36 \quad 56 \quad 44-9999$

070305A $1985 \quad 198 \quad 436 \quad 412 \quad 346 \quad 358$

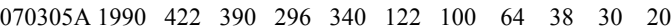

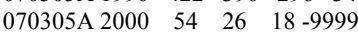

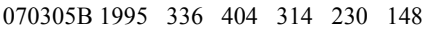

070305B $2000 \quad 360 \quad 326 \quad 70-9999$

070306A $1975 \quad 114 \quad 224 \quad 156 \quad 246 \quad 254$

$\begin{array}{llllllllll}\text { 070306A } 1981 & 186 & 288 & 164 & 198 & 170 & 114 & 158 & 80 & 82\end{array}$

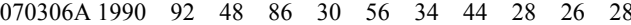

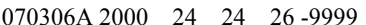

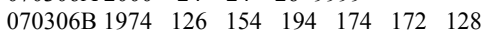




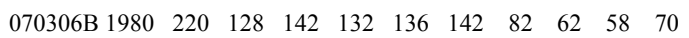

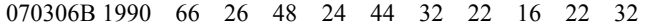
070306B $2000 \quad 30 \quad 20 \quad 26-9999$

070307A $1975 \quad 278 \quad 112 \quad 98 \quad 112 \quad 86$

070307A 1980 $90 \begin{array}{llllllllll}90 & 168 & 122 & 86 & 60 & 48 & 58 & 58 & 76 & 72\end{array}$ $\begin{array}{llllllllllll}070307 \mathrm{~A} & 1990 & 110 & 76 & 42 & 120 & 78 & 84 & 26 & 60 & 38 & 72\end{array}$ 070307A $2000 \quad 82 \quad 46 \quad 46-9999$

070307B $1975 \quad 158 \quad 116 \quad 146 \quad 106 \quad 92$

070307B 1980 $1100 \begin{array}{llllllllll}170 & 64 & 74 & 54 & 48 & 70 & 40 & 60 & 72\end{array}$

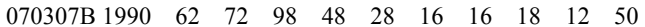

$\begin{array}{lllll}070307 \mathrm{~B} 2000 & 70 & 88 & 28 & -9999\end{array}$

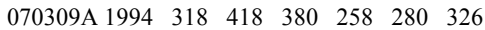

070309A $2000 \quad 178 \quad 116 \quad 92-9999$

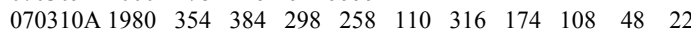

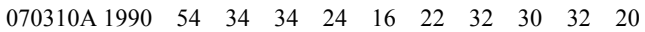

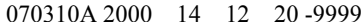

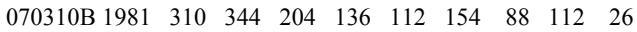

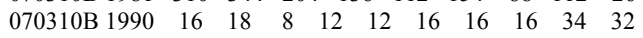

070310B 2000 $24 \quad 26 \quad 14-9999$

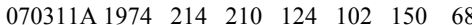

$\begin{array}{lllllllllll}070311 \text { A } 1980 & 106 & 96 & 116 & 94 & 12 & 78 & 46 & 46 & 42 & 88\end{array}$

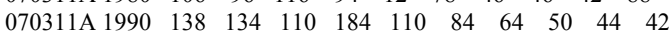

070311A $2000 \quad 38 \quad 60 \quad 40-9999$

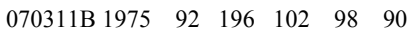

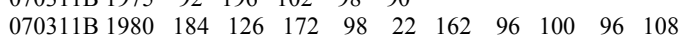

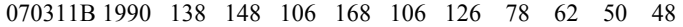
070311B $2000 \quad 40 \quad 68 \quad 42-9999$

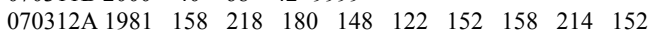

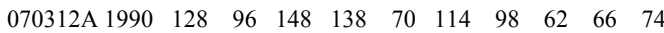

070312A $2000 \quad 94 \quad 70 \quad 46-9999$

070313A 1989296

$\begin{array}{lllllllllll}070313 \mathrm{~A} 1990 & 522 & 370 & 384 & 464 & 384 & 314 & 198 & 204 & 170 & 78\end{array}$

070313A $2000 \quad 46 \quad 34 \quad 36-9999$

$\begin{array}{lllllllllll}070314 \mathrm{~A} 1980 & 68 & 158 & 148 & 208 & 190 & 184 & 184 & 218 & 186 & 112\end{array}$

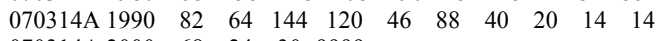

070314A $2000 \quad 68 \quad 24 \quad 30-9999$

070314B $1985 \quad 58 \quad 128 \quad 116 \quad 152 \quad 142$

$\begin{array}{lllllllllll}070314 \mathrm{~B} 1990 & 250 & 160 & 160 & 164 & 198 & 196 & 142 & 92 & 66 & 178\end{array}$

070314B 2000 $154 \quad 128 \quad 54-9999$

070315A 1979216

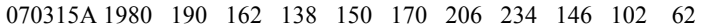

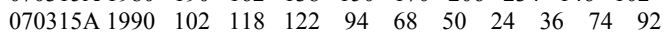

070315A $2000 \quad 82 \quad 32 \quad 40-9999$

070315B $1978 \quad 44 \quad 258$

$\begin{array}{llllllllllll}070315 B & 1980 & 186 & 182 & 190 & 186 & 174 & 174 & 186 & 146 & 78 & 58\end{array}$

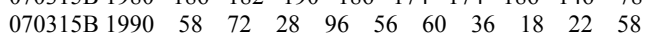

070315B $2000 \quad 72 \quad 46 \quad 24-9999$

070316A $1986 \quad 492 \quad 622 \quad 462 \quad 284$

$\begin{array}{lllllllllll}070316 \text { A } 1990 & 288 & 186 & 180 & 190 & 156 & 138 & 58 & 26 & 44 & 26\end{array}$

070316A $2000 \quad 26 \quad 26 \quad 28-9999$

070316B $1993 \quad 608 \quad 506 \quad 264 \quad 352 \quad 208 \quad 204 \quad 302$

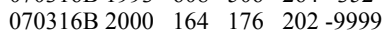

070317A 1979326

070317A 1980 $574 \begin{array}{llllllllll}548 & 576 & 450 & 302 & 350 & 284 & 192 & 212 & 144\end{array}$

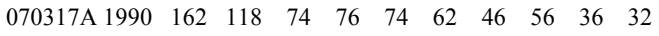

070317A $2000 \quad 32 \quad 40 \quad 52-9999$

070317B $1975 \quad 336 \quad 162 \quad 248 \quad 204 \quad 248$

$\begin{array}{lllllllllll}070317 \mathrm{~B} 1980 & 260 & 480 & 406 & 218 & 276 & 204 & 180 & 214 & 148 & 144\end{array}$

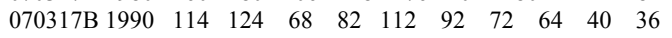

070317B $2000 \quad 32 \quad 36 \quad 84-9999$

$\begin{array}{lllll}070318 \text { A } 1987 & 178 & 414 & 580\end{array}$

$\begin{array}{llllllllllll}\text { 070318A } 1990 & 646 & 442 & 290 & 380 & 366 & 232 & 176 & 124 & 108 & 124\end{array}$

070318A $2000 \quad 54 \quad 82 \quad 78-9999$

070318B $1988 \quad 196 \quad 132$

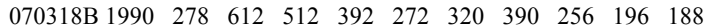

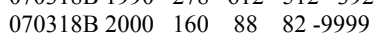

070319A $1975 \quad 244 \quad 236 \quad 182 \quad 166 \quad 82$

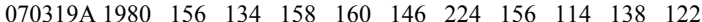

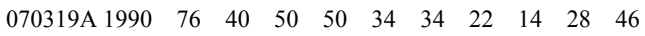

070319A $2000 \quad 42 \quad 32 \quad 26-9999$

070319B $1977 \quad 302 \quad 162 \quad 140$

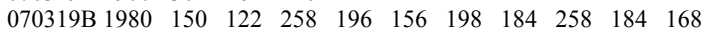

$\begin{array}{llllllllllll}070319 \mathrm{~B} & 1990 & 166 & 154 & 110 & 60 & 70 & 58 & 40 & 26 & 80 & 38\end{array}$

070319B 2000 $52 \quad 44 \quad 30-9999$

070320A $1976 \quad 218 \quad 804 \quad 736 \quad 1042$

$\begin{array}{lllllllllll}070320 \text { A } 1980 & 994 & 1120 & 1102 & 948 & 1384 & 1166 & 732 & 190 & 178 & 786\end{array}$

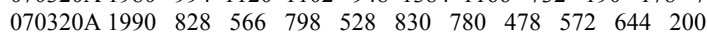

070320A $2000 \quad 378 \quad 482 \quad 138-9999$

070320B $1982 \quad 340 \quad 984 \quad 1202 \quad 990 \quad 358 \quad 348 \quad 836 \quad 652$

$\begin{array}{lllllllllll}070320 \mathrm{~B} 1990 & 342 & 758 & 464 & 696 & 854 & 686 & 802 & 742 & 300 & 322\end{array}$
070320B $2000 \quad 436 \quad 198 \quad 158-9999$

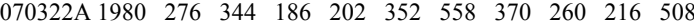
$\begin{array}{llllllllllll}070322 \text { A } 1990 & 616 & 336 & 554 & 368 & 464 & 470 & 356 & 286 & 292 & 174\end{array}$ 070322A $2000 \quad 178 \quad 320 \quad 182-9999$

$\begin{array}{llllll}070322 \mathrm{~B} & 1976 & 224 & 236 & 148 & 120\end{array}$

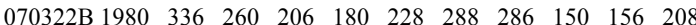

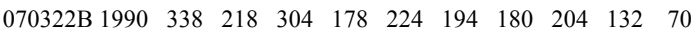
070322B $2000 \quad 102 \quad 138 \quad 60-9999$

$\begin{array}{llllllllll}070323 \mathrm{~A} 1971 & 50 & 158 & 110 & 288 & 454 & 498 & 676 & 586 & 582\end{array}$

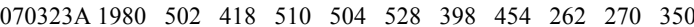

$\begin{array}{lllllllllll}070323 \text { A } 1990 & 312 & 284 & 360 & 228 & 238 & 140 & 178 & 236 & 202 & 90\end{array}$ 070323A $2000 \quad 194 \quad 94 \quad 78-9999$

070323B $1972 \quad 128 \quad 292 \quad 276 \quad 344 \quad 462 \quad 692 \quad 592 \quad 572$

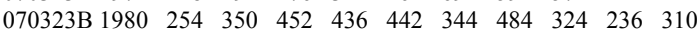

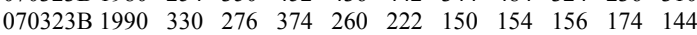

070323B $2000 \quad 210 \quad 164 \quad 84-9999$

070324A $1978 \quad 1994 \quad 678$

070324A 1980 $296 \begin{array}{llllllllll}326 & 362 & 430 & 464 & 608 & 432 & 140 & 58 & 362\end{array}$

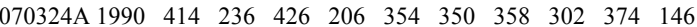
070324A $2000 \quad 348 \quad 462 \quad 110-9999$

070324B $1977 \quad 360 \quad 9181016$

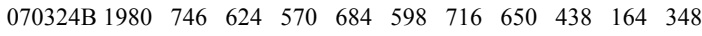

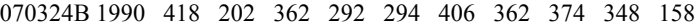
070324B 2000 $300 \quad 460 \quad 196-9999$

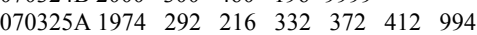

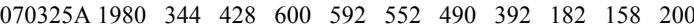

$\begin{array}{lllllllllll}070325 \mathrm{~A} 1990 & 232 & 206 & 324 & 224 & 240 & 138 & 252 & 204 & 138 & 118\end{array}$

070325A $2000 \quad 160 \quad 136 \quad 54-9999$

$\begin{array}{llllll}070325 B & 1976 & 254 & 258 & 290 & 252\end{array}$

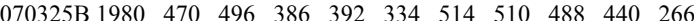

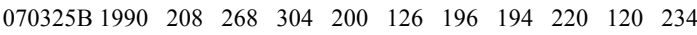
070325B $2000 \quad 98 \quad 126 \quad 64-9999$

070326A $1976 \quad 236 \quad 616 \quad 656 \quad 666$

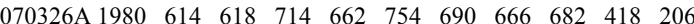

$\begin{array}{llllllllllll}070326 \mathrm{~A} & 1990 & 340 & 350 & 170 & 408 & 158 & 290 & 352 & 368 & 374 & 382\end{array}$

070326A $2000 \quad 378 \quad 306 \quad 114-9999$

070326B $1977 \quad 198 \quad 802 \quad 630$

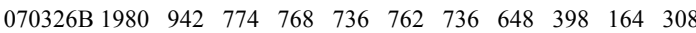

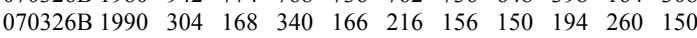

070326B $2000 \quad 290 \quad 408 \quad 132-9999$

070327A $1983 \quad 118 \quad 1216 \quad 1168 \quad 726 \quad 990 \quad 1042 \quad 774$

$\begin{array}{lllllllllll}070327 \mathrm{~A} 1990 & 724 & 652 & 432 & 608 & 456 & 516 & 628 & 530 & 462 & 502\end{array}$ 070327A $2000 \quad 330 \quad 172 \quad 120-9999$

070327B $1976 \quad 150 \quad 226 \quad 276 \quad 220$

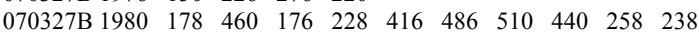

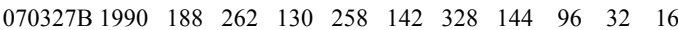

070327B $2000 \quad 8 \quad 16 \quad 22 \quad-9999$

070328A $1985 \quad 372 \quad 472 \quad 328 \quad 276 \quad 494$

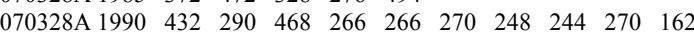
070328A $2000 \quad 418 \quad 472 \quad 154-9999$

$\begin{array}{llllll}070328 \mathrm{~B} & 1986 & 94 & 570 & 252 & 482\end{array}$

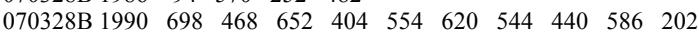

070328B $2000 \quad 308 \quad 440 \quad 178-9999$

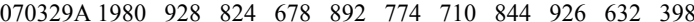

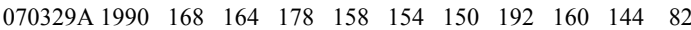

070329A $2000 \quad 164 \quad 270 \quad 94-9999$

070329B 1979396

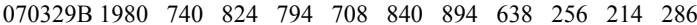

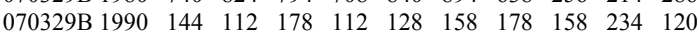
070329B 2000 $166 \quad 268 \quad 104-9999$

$\begin{array}{lllllllllll}070330 \text { A } 1980 & 354 & 434 & 390 & 436 & 368 & 208 & 288 & 456 & 258 & 276\end{array}$

070330A 1990 $27 \begin{array}{llllllllll} & 130 & 148 & 66 & 52 & 66 & 38 & 32 & 36 & 44\end{array}$

070330A $2000 \quad 46 \quad 46 \quad 50-9999$

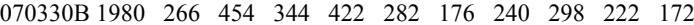

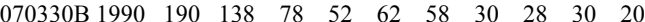

070330B $2000 \quad 28 \quad 36 \quad 32-9999$

070331A $1973 \quad 246 \quad 760 \quad 594 \quad 568 \quad 446 \quad 334 \quad 408$

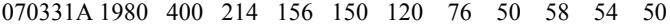

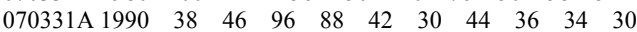

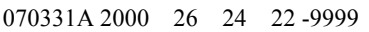

070331B $1973 \quad 294 \quad 662 \quad 652 \quad 716 \quad 708 \quad 502 \quad 462$

$\begin{array}{lllllllllll}070331 \mathrm{~B} 1980 & 442 & 260 & 196 & 134 & 182 & 118 & 68 & 66 & 64 & 70\end{array}$

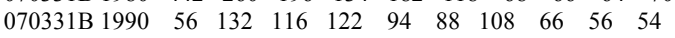

070331B $2000 \quad 58 \quad 66 \quad 68-9999$

070332 A $1979 \quad 300$

$\begin{array}{lllllllllll}070332 \mathrm{~A} 1980 & 504 & 418 & 304 & 246 & 454 & 444 & 280 & 122 & 128 & 394\end{array}$

$\begin{array}{lllllllllll}070332 \mathrm{~A} 1990 & 446 & 272 & 424 & 268 & 382 & 322 & 252 & 110 & 94 & 72\end{array}$

070332A $2000 \quad 158 \quad 274 \quad 80-9999$

070332B $1983 \quad 544 \quad 406 \quad 468 \quad 370 \quad 216 \quad 256 \quad 742$

$\begin{array}{lllllllllll}070332 \mathrm{~B} 1990 & 614 & 370 & 608 & 410 & 436 & 488 & 340 & 246 & 120 & 86\end{array}$ 
070332B $2000 \quad 294 \quad 380 \quad 116-9999$

070333A $1975 \quad 82 \quad 366 \quad 558 \quad 552 \quad 346$

$\begin{array}{lllllllllll}070333 \mathrm{~A} 1980 & 312 & 702 & 570 & 590 & 500 & 672 & 922 & 344 & 236 & 468\end{array}$ $\begin{array}{lllllllllll}070333 \mathrm{~A} 1990 & 524 & 422 & 546 & 354 & 306 & 218 & 156 & 134 & 122 & 92\end{array}$ 070333A $2000 \quad 100 \quad 66 \quad 60-9999$

070333B $1976 \quad 72 \quad 460 \quad 670 \quad 650$

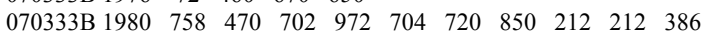

$\begin{array}{lllllllllll}070333 \mathrm{~B} 1990 & 430 & 346 & 570 & 382 & 370 & 406 & 468 & 360 & 336 & 178\end{array}$

070333B $2000 \quad 280 \quad 204 \quad 112-9999$

070334A $1975 \quad 232 \quad 106 \quad 144 \quad 102 \quad 196$

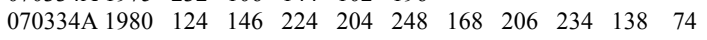

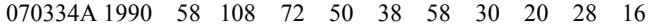

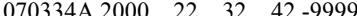

070334B $1978 \quad 210 \quad 156$

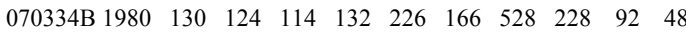

070334B 1990 $440 \begin{array}{llllllllll}80 & 44 & 24 & 34 & 24 & 34 & 30 & 28 & 18\end{array}$

070334B 2000 $\quad 18 \quad 30 \quad 46-9999$

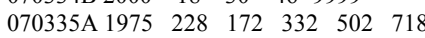

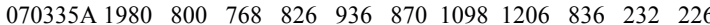

$\begin{array}{llllllllllll}070335 \mathrm{~A} 1990 & 692 & 774 & 550 & 616 & 380 & 598 & 518 & 592 & 444 & 602\end{array}$

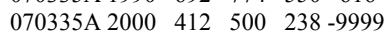

070335B $1977 \quad 780 \quad 718 \quad 938$

$\begin{array}{llllllllllll}070335 \mathrm{~B} & 1980 & 656 & 814 & 924 & 924 & 1050 & 1198 & 858 & 256 & 204 & 826\end{array}$

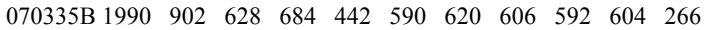

070335B $2000 \quad 452 \quad 550 \quad 150-9999$

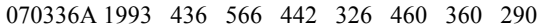

$\begin{array}{llllll}070336 \text { A } 2000 & 346 & 206 & 218 & -9999\end{array}$

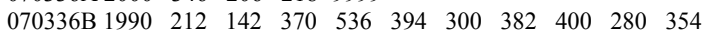

070336B $2000 \quad 190 \quad 164 \quad 200-9999$

070337A $1975 \quad 304 \quad 176 \quad 126 \quad 104 \quad 82$

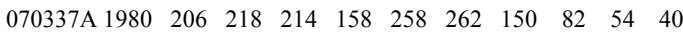

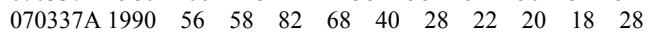

070337A $2000 \quad 26 \quad 26 \quad 26-9999$

$\begin{array}{lllll}070337 \mathrm{~B} 1976 & 78 & 186 & 158 & 168\end{array}$

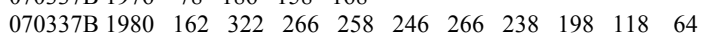

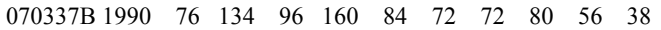

070337B $2000 \quad 60 \quad 58 \quad 60-9999$

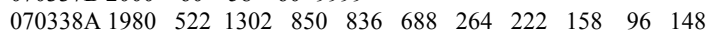

$\begin{array}{lllllllllll}070338 \text { A } 1990 & 222 & 166 & 296 & 178 & 238 & 268 & 258 & 278 & 244 & 180\end{array}$

070338A $2000 \quad 200 \quad 358 \quad 156-9999$

070338B 1979226

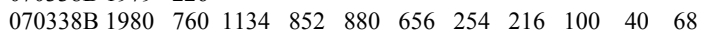

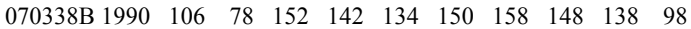

070338B $2000 \quad 142 \quad 276 \quad 110-9999$

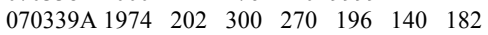

$\begin{array}{lllllllllll}\text { 070339A } 1980 & 162 & 122 & 120 & 142 & 192 & 144 & 88 & 146 & 162 & 154\end{array}$

$\begin{array}{llllllllllll}070339 \text { A } 1990 & 150 & 234 & 328 & 180 & 224 & 92 & 138 & 134 & 252 & 210\end{array}$

070339A $2000 \quad 216 \quad 182 \quad 138 \quad-9999$

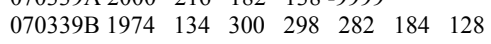

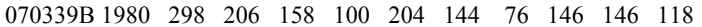

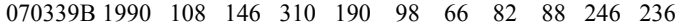

070339B 2000 $206 \quad 120 \quad 110-9999$

070340A $1977 \quad 80 \quad 1018 \quad 798$

$\begin{array}{lllllllllll}070340 \text { A } 1980 & 1156 & 746 & 834 & 948 & 640 & 218 & 110 & 82 & 26 & 44\end{array}$

$\begin{array}{llllllllllll}070340 \mathrm{~A} & 1990 & 98 & 74 & 136 & 124 & 166 & 176 & 134 & 158 & 210 & 110\end{array}$

070340A $2000 \quad 122 \quad 144 \quad 48-9999$

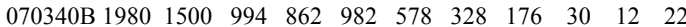

$\begin{array}{lllllllllll}070340 \mathrm{~B} 1990 & 48 & 60 & 80 & 70 & 90 & 90 & 86 & 74 & 62 & 48\end{array}$

070340B $2000 \quad 50 \quad 46 \quad 68-9999$

$\begin{array}{lllllllllll}070341 \text { A } 1980 & 322 & 216 & 250 & 246 & 210 & 210 & 196 & 174 & 162 & 238\end{array}$

$\begin{array}{lllllllllll}070341 \mathrm{~A} 1990 & 206 & 162 & 226 & 150 & 108 & 82 & 50 & 48 & 26 & 86\end{array}$

070341A $2000 \quad 106 \quad 78 \quad 40-9999$

070341B $1979 \quad 58$

$\begin{array}{lllllllllll}070341 \mathrm{~B} 1980 & 134 & 242 & 244 & 230 & 258 & 232 & 196 & 284 & 208 & 168\end{array}$

$\begin{array}{llllllllllll}070341 \mathrm{~B} & 1990 & 112 & 196 & 226 & 246 & 196 & 202 & 88 & 114 & 82 & 90\end{array}$

070341B $2000 \quad 90 \quad 92 \quad 56-9999$

070342A $1978 \quad 258 \quad 190$

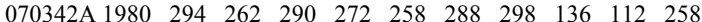

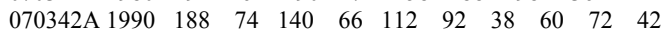

070342A $2000 \quad 88 \quad 40 \quad 84-9999$

070342B $1977 \quad 142 \quad 296 \quad 214$

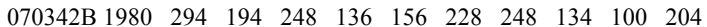

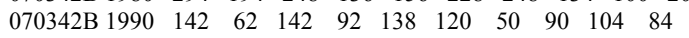

070342B $2000 \quad 90 \quad 104 \quad 40-9999$

070401A $1973 \quad 38 \quad 400 \quad 318 \quad 336 \quad 460 \quad 530 \quad 456$

$\begin{array}{lllllllllll}070401 \mathrm{~A} 1980 & 620 & 804 & 592 & 278 & 176 & 542 & 614 & 324 & 264 & 354\end{array}$

$\begin{array}{llllllllllll}070401 \mathrm{~A} 1990 & 364 & 312 & 202 & 198 & 180 & 164 & 136 & 134 & 174 & 94\end{array}$

070401A $2000 \quad 276 \quad 232 \quad 174-9999$

070401B $1972 \quad 164 \quad 326 \quad 272 \quad 358 \quad 296 \quad 228 \quad 342 \quad 300$

$\begin{array}{lllllllllll}070401 \mathrm{~B} 1980 & 376 & 472 & 438 & 410 & 418 & 404 & 380 & 268 & 152 & 286\end{array}$ $\begin{array}{lllllllllll}070401 \mathrm{~B} 1990 & 284 & 144 & 166 & 122 & 80 & 96 & 128 & 182 & 258 & 102\end{array}$ 070401B $2000 \quad 252 \quad 268 \quad 156-9999$

$\begin{array}{llllllllll}070402 \mathrm{~A} 1971 & 164 & 248 & 330 & 400 & 510 & 356 & 366 & 366 & 276\end{array}$

$\begin{array}{llllllllllll}070402 \mathrm{~A} 1980 & 422 & 426 & 484 & 428 & 400 & 450 & 246 & 164 & 112 & 198\end{array}$ $\begin{array}{lllllllllll}070402 \text { A } 1990 & 172 & 146 & 146 & 148 & 162 & 180 & 166 & 138 & 168 & 78\end{array}$

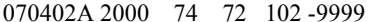

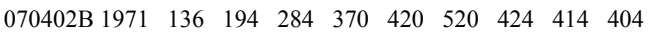

$\begin{array}{llllllllllll}070402 B & 1980 & 354 & 416 & 350 & 368 & 412 & 390 & 386 & 232 & 146 & 150\end{array}$

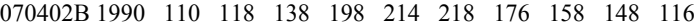
070402B $2000 \quad 124 \quad 162 \quad 118-9999$

070403A $1987 \quad 186 \quad 292 \quad 350$

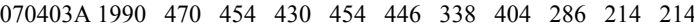
070403A $2000 \quad 164 \quad 122 \quad 106-9999$

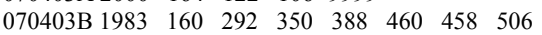

$\begin{array}{llllllllllll}070403 \mathrm{~B} & 1990 & 610 & 442 & 450 & 442 & 270 & 218 & 204 & 166 & 124 & 88\end{array}$

070403B $2000 \quad 62 \quad 44 \quad 24-9999$

070404A $1976 \quad 150 \quad 278 \quad 362 \quad 450$

$\begin{array}{llllllllllll}070404 \text { A } 1980 & 228 & 380 & 364 & 432 & 436 & 476 & 330 & 268 & 294 & 272\end{array}$

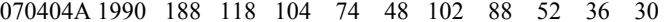

070404A $2000 \quad 22 \quad 24 \quad 38-9999$

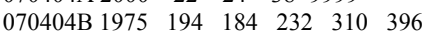

$\begin{array}{llllllllllll}070404 \mathrm{~B} 1980 & 416 & 270 & 398 & 402 & 366 & 322 & 228 & 174 & 174 & 192\end{array}$

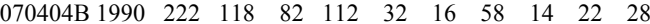

070404B 2000 $36 \quad 20 \quad 34-9999$

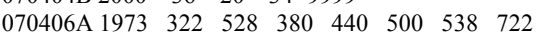

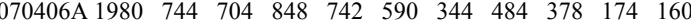

$\begin{array}{lllllllllll}070406 \text { A } 1990 & 244 & 192 & 376 & 222 & 366 & 402 & 278 & 174 & 192 & 142\end{array}$

070406A $2000 \quad 340 \quad 252 \quad 124-9999$

$\begin{array}{lllllllll}070406 \mathrm{~B} & 1973 & 338 & 596 & 426 & 490 & 592 & 798 & 744\end{array}$

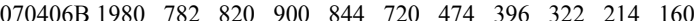

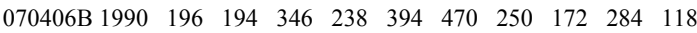

070406B 2000 $172 \quad 250 \quad 148-9999$

070408A $1985 \quad 110 \quad 302 \quad 456 \quad 564 \quad 236$

$\begin{array}{lllllllllll}070408 \text { A } 1990 & 400 & 276 & 198 & 366 & 256 & 334 & 300 & 300 & 270 & 332\end{array}$

070408A $2000 \quad 326 \quad 280 \quad 182-9999$

070408B $1988 \quad 546 \quad 244$

$\begin{array}{llllllllllll}070408 \mathrm{~B} 1990 & 496 & 356 & 392 & 198 & 126 & 236 & 268 & 282 & 458 & 408\end{array}$ 070408B $2000 \quad 278 \quad 468 \quad 128-9999$

$\begin{array}{lllllllllll}070411 \text { A } 1970 & 246 & 184 & 140 & 162 & 276 & 234 & 180 & 100 & 196 & 88\end{array}$

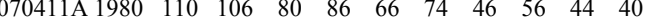

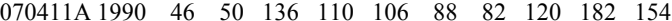

070411A $2000 \quad 204 \quad 152 \quad 86-9999$

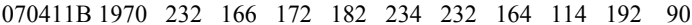

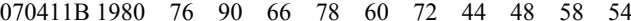

070411B 1990 $56 \begin{array}{llllllllll}56 & 38 & 142 & 106 & 94 & 86 & 82 & 92 & 174 & 156\end{array}$

070411B 2000 $232 \quad 180 \quad 114-9999$

070412A $1985 \quad 140 \quad 486 \quad 484 \quad 490 \quad 428$

$\begin{array}{lllllllllll}070412 \text { A } 1990 & 382 & 340 & 256 & 232 & 292 & 238 & 254 & 150 & 70 & 86\end{array}$

070412A $2000 \quad 92 \quad 132 \quad 42-9999$

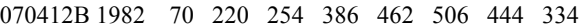

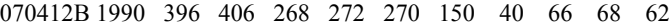

070412B $2000 \quad 70 \quad 64 \quad 40-9999$

$\begin{array}{llllllllll}070413 \text { A } 1971 & 110 & 580 & 406 & 810 & 874 & 888 & 788 & 862 & 926\end{array}$

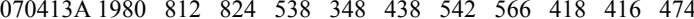

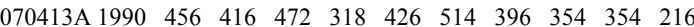

070413A 2000 $230 \quad 196 \quad 162-9999$

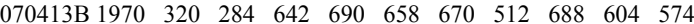

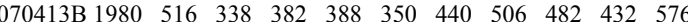
$\begin{array}{lllllllllll}070413 \mathrm{~B} 1990 & 356 & 294 & 430 & 470 & 254 & 576 & 608 & 432 & 370 & 326\end{array}$ 070413B $2000 \quad 338 \quad 360 \quad 222-9999$

070415A $1974 \quad 466 \quad 542 \quad 342 \quad 320 \quad 576 \quad 662$

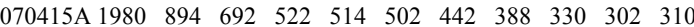

$\begin{array}{llllllllllll}070415 \text { A } 1990 & 330 & 270 & 422 & 290 & 344 & 268 & 214 & 158 & 140 & 90\end{array}$ 070415A $2000 \quad 106 \quad 192 \quad 146-9999$

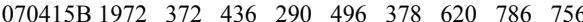

070415B $1980 \begin{array}{lllllllllll}906 & 796 & 772 & 654 & 656 & 556 & 586 & 432 & 434 & 504\end{array}$ $\begin{array}{lllllllllll}070415 \mathrm{~B} 1990 & 460 & 368 & 458 & 286 & 392 & 310 & 228 & 200 & 210 & 170\end{array}$

070415B $2000 \quad 150 \quad 300 \quad 206-9999$

$\begin{array}{llll}070416 \text { A } 1987 & 84 & 248 & 332\end{array}$

$\begin{array}{lllllllllll}070416 \text { A } 1990 & 460 & 480 & 362 & 378 & 282 & 208 & 212 & 160 & 144 & 134\end{array}$ 070416A $2000 \quad 74 \quad 56 \quad 50-9999$

070416B $1986 \quad 166 \quad 330 \quad 338 \quad 382$

$\begin{array}{lllllllllll}070416 \mathrm{~B} 1990 & 438 & 380 & 402 & 226 & 226 & 172 & 114 & 176 & 128 & 78\end{array}$

070416B $2000 \quad 54 \quad 48 \quad 36-9999$

070417A $1977 \quad 132 \quad 170 \quad 524$

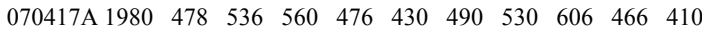

$\begin{array}{lllllllllll}070417 \text { A } 1990 & 288 & 310 & 290 & 310 & 240 & 168 & 250 & 102 & 178 & 98\end{array}$

070417A $2000 \quad 78 \quad 28 \quad 36-9999$

070417B $1979 \quad 82$

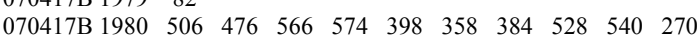


$\begin{array}{lllllllllll}070417 \mathrm{~B} 1990 & 308 & 170 & 226 & 174 & 178 & 114 & 154 & 118 & 60 & 82\end{array}$ 070417B $2000 \quad 76 \quad 50 \quad 46-9999$

070418A $1978 \quad 238 \quad 630$

$\begin{array}{lllllllllll}\text { 070418A } 1980 & 358 & 316 & 298 & 292 & 252 & 494 & 468 & 376 & 276 & 372\end{array}$

$\begin{array}{llllllllllll}070418 \text { A } 1990 & 368 & 310 & 308 & 244 & 178 & 76 & 82 & 64 & 78 & 98\end{array}$

070418A $2000 \quad 38 \quad 38 \quad 62-9999$

070418B 1979318

$\begin{array}{lllllllllll}070418 \mathrm{~B} 1980 & 606 & 270 & 284 & 282 & 202 & 200 & 304 & 308 & 208 & 206\end{array}$

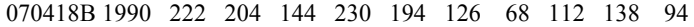

070418B $2000 \quad 50 \quad 56 \quad 36-9999$

$\begin{array}{lllllllllll}\text { 070419A } 1980 & 168 & 420 & 454 & 516 & 656 & 926 & 828 & 528 & 312 & 260\end{array}$

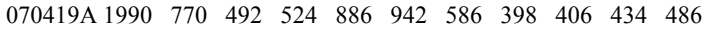
070419A $2000 \quad 592 \quad 556 \quad 284-9999$

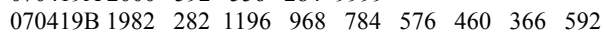

$\begin{array}{lllllllllll}070419 \mathrm{~B} 1990 & 462 & 404 & 480 & 324 & 960 & 438 & 302 & 318 & 368 & 394\end{array}$ 070419B 2000 $394 \quad 436 \quad 224-9999$

070420A $1975 \quad 212 \quad 218 \quad 470 \quad 446 \quad 310$

$\begin{array}{llllllllllll}070420 \text { A } 1980 & 366 & 372 & 262 & 218 & 186 & 176 & 168 & 88 & 62 & 66\end{array}$

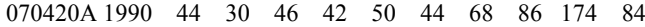

070420A $2000 \quad 172 \quad 128 \quad 106-9999$

$\begin{array}{llllll}070420 \mathrm{~B} & 1976 & 112 & 48 & 244 & 172\end{array}$

$\begin{array}{lllllllllll}070420 \mathrm{~B} 1980 & 450 & 448 & 348 & 458 & 440 & 386 & 278 & 260 & 252 & 172\end{array}$

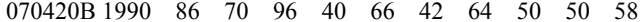

070420B 2000 $128 \quad 102 \quad 132-9999$

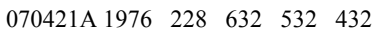

070421A $1980 \quad 556 \quad 802 \quad 788 \quad 662 \quad 536 \quad 464 \quad 582 \quad 458 \quad 466 \quad 462$

$\begin{array}{lllllllllll}070421 \mathrm{~A} 1990 & 332 & 272 & 310 & 354 & 476 & 304 & 354 & 276 & 296 & 150\end{array}$

070421A $2000 \quad 262 \quad 198 \quad 154-9999$

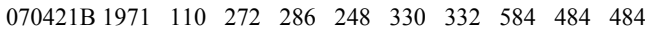

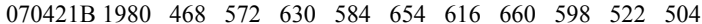

$\begin{array}{lllllllllll}070421 \mathrm{~B} 1990 & 500 & 478 & 548 & 466 & 594 & 450 & 438 & 396 & 424 & 304\end{array}$

070421B $2000 \quad 300 \quad 316 \quad 250-9999$

070422A $1976 \quad 314 \quad 732 \quad 624 \quad 392$

$\begin{array}{lllllllllll}070422 \mathrm{~A} 1980 & 506 & 574 & 582 & 638 & 222 & 288 & 296 & 376 & 228 & 200\end{array}$

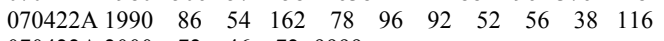

070422A $2000 \quad 72 \quad 46 \quad 72-9999$

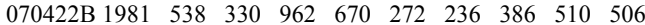

$\begin{array}{llllllllllll}070422 \mathrm{~B} & 1990 & 234 & 422 & 214 & 216 & 254 & 74 & 160 & 102 & 70 & 70\end{array}$

070422B $2000 \quad 76 \quad 114 \quad 108-9999$

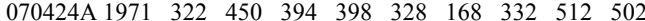

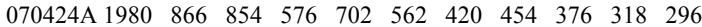

$\begin{array}{lllllllllll}070424 \mathrm{~A} 1990 & 242 & 192 & 206 & 228 & 300 & 354 & 266 & 246 & 252 & 140\end{array}$

070424A $2000 \quad 110 \quad 178 \quad 118-9999$

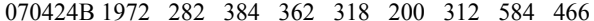

$\begin{array}{lllllllllll}070424 \mathrm{~B} 1980 & 722 & 688 & 522 & 486 & 438 & 464 & 356 & 444 & 492 & 370\end{array}$

$\begin{array}{llllllllllll}070424 \mathrm{~B} 1990 & 394 & 410 & 474 & 302 & 288 & 290 & 156 & 130 & 184 & 100\end{array}$

070424B 2000 $114 \quad 120 \quad 66-9999$

070425A 1979232

$\begin{array}{lllllllllll}070425 \text { A } 1980 & 504 & 338 & 264 & 268 & 320 & 344 & 250 & 444 & 486 & 298\end{array}$

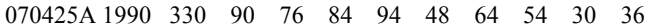

070425A $2000 \quad 40 \quad 28 \quad 30-9999$

070425 B $1979 \quad 158$

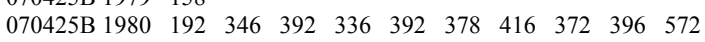

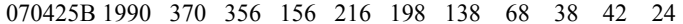

070425B 2000 $20 \quad 20 \quad 20-9999$

$\begin{array}{lllllllllll}070426 \text { A } 1980 & 304 & 302 & 236 & 420 & 240 & 168 & 196 & 206 & 282 & 316\end{array}$

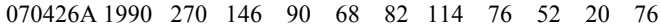

070426A $2000 \quad 46 \quad 40 \quad 42-9999$

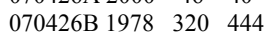

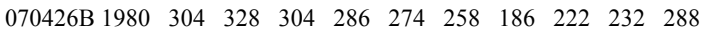

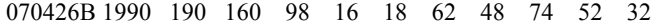

070426B $2000 \quad 40 \quad 36 \quad 50-9999$

070427A $1986 \quad 516 \quad 498 \quad 268 \quad 220$

$\begin{array}{lllllllllll}070427 \mathrm{~A} 1990 & 234 & 174 & 230 & 272 & 212 & 264 & 174 & 152 & 120 & 94\end{array}$

070427A $2000 \quad 80 \quad 50 \quad 66-9999$

$\begin{array}{llllllll}070427 \mathrm{~B} 1983 & 88 & 362 & 464 & 282 & 264 & 276 & 250\end{array}$

$\begin{array}{lllllllllll}070427 \mathrm{~B} 1990 & 298 & 330 & 216 & 426 & 136 & 168 & 166 & 112 & 78 & 72\end{array}$

070427B $2000 \quad 52 \quad 36 \quad 30-9999$

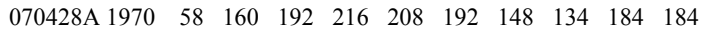

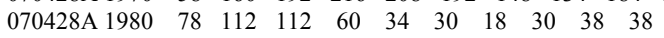

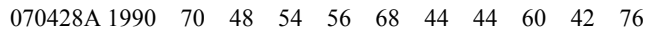

070428A $2000 \quad 70 \quad 54 \quad 42-9999$

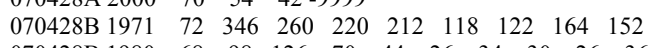

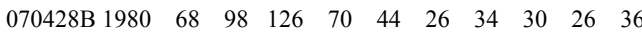

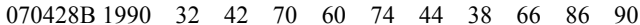

070428B $2000 \quad 76 \quad 52 \quad 32-9999$

070429A 1979298

$\begin{array}{lllllllllll}070429 \text { A } 1980 & 304 & 162 & 164 & 182 & 128 & 194 & 208 & 140 & 120 & 90\end{array}$

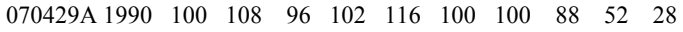

070429A $2000 \quad 32 \quad 44 \quad 44-9999$
070429B $1977 \quad 102 \quad 218 \quad 246$

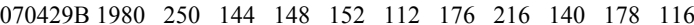

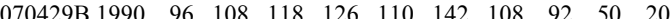

070429B $2000 \quad 32 \quad 34 \quad 34-9999$

$\begin{array}{llllllll}070430 \text { A } 1983 & 212 & 294 & 246 & 242 & 206 & 238 & 220\end{array}$

$\begin{array}{llllllllllll}070430 \mathrm{~A} & 1990 & 156 & 256 & 290 & 182 & 246 & 126 & 94 & 120 & 74 & 90\end{array}$

070430A $2000 \quad 60 \quad 36 \quad 20-9999$

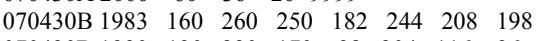

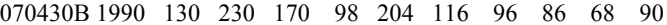

070430B $2000 \quad 44 \quad 38 \quad 36-9999$

$\begin{array}{lllllll}070431 \text { A } 1974 & 130 & 230 & 182 & 94 & 64 & 92\end{array}$

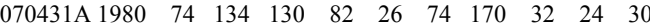

$\begin{array}{lllllllllll}070431 \mathrm{~A} 1990 & 32 & 62 & 72 & 54 & 58 & 48 & 64 & 110 & 114 & 78\end{array}$

070431A $2000 \quad 108 \quad 90 \quad 20-9999$

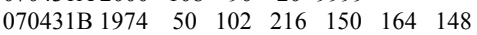

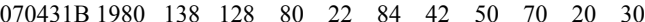

$\begin{array}{lllllllllll}070431 \mathrm{~B} 1990 & 30 & 30 & 76 & 44 & 56 & 56 & 78 & 82 & 82 & 80\end{array}$

070431B $2000 \quad 54 \quad 84 \quad 36-9999$

070432A $1969 \quad 84$

$\begin{array}{lllllllllll}070432 \text { A } 1970 & 320 & 222 & 238 & 218 & 184 & 220 & 154 & 186 & 176 & 108\end{array}$

$\begin{array}{llllllllllll}070432 \mathrm{~A} & 1980 & 234 & 198 & 172 & 104 & 146 & 152 & 180 & 124 & 86 & 100\end{array}$

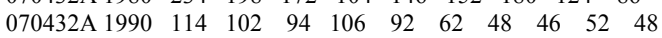

070432A $2000 \quad 62 \quad 90 \quad 54-9999$

070432B 196972

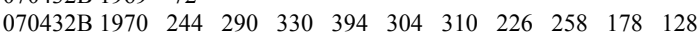

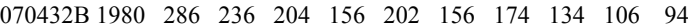

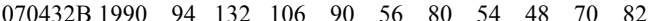

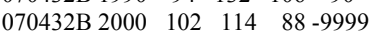

$\begin{array}{lllllllllll}070433 \text { A } 1980 & 234 & 296 & 294 & 228 & 146 & 130 & 230 & 238 & 180 & 250\end{array}$

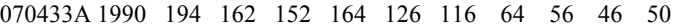

070433A $2000 \quad 38 \quad 24 \quad 32-9999$

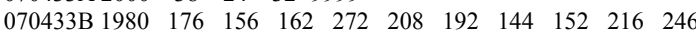

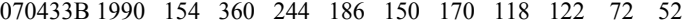

070433B $2000 \quad 48 \quad 46 \quad 42-9999$

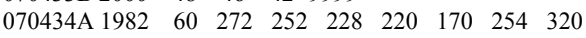

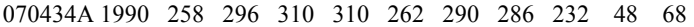

070434A $2000 \quad 94 \quad 66 \quad 78-9999$

070434B $1979 \quad 62$

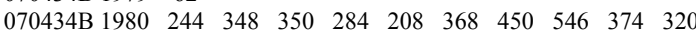

$\begin{array}{lllllllllll}070434 \mathrm{~B} 1990 & 334 & 334 & 372 & 322 & 332 & 82 & 70 & 58 & 28 & 28\end{array}$

070434B 2000 $28 \quad 30 \quad 30-9999$

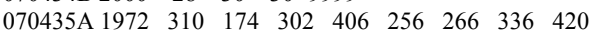

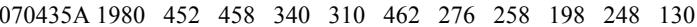

$\begin{array}{llllllllllll}070435 \mathrm{~A} 1990 & 210 & 254 & 216 & 262 & 146 & 146 & 142 & 110 & 218 & 78\end{array}$

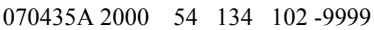

$\begin{array}{lllllllllll}070435 \mathrm{~B} 1970 & 262 & 412 & 128 & 396 & 334 & 356 & 318 & 324 & 470 & 492\end{array}$

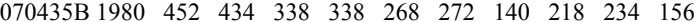

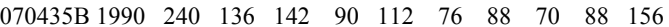

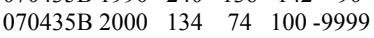

070436A 1971 $312 \quad 320 \quad 262 \quad 258 \quad 256 \quad 176 \quad 234 \quad 242 \quad 312$

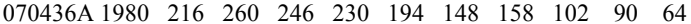

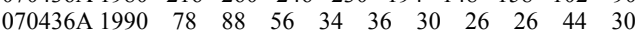

070436A $2000 \quad 68 \quad 64 \quad 64-9999$

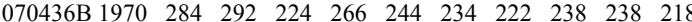

$\begin{array}{llllllllllll}070436 \mathrm{~B} & 1980 & 234 & 252 & 280 & 160 & 152 & 180 & 194 & 166 & 98 & 98\end{array}$

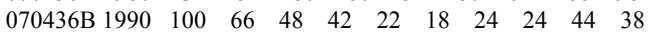

070436B $2000 \quad 64 \quad 56 \quad 58-9999$

070437A $1974 \quad 194 \quad 444 \quad 442 \quad 296 \quad 328 \quad 300$

$\begin{array}{lllllllllll}070437 \text { A } 1980 & 298 & 242 & 310 & 352 & 338 & 338 & 236 & 210 & 174 & 222\end{array}$

$\begin{array}{lllllllllll}070437 \text { A } 1990 & 150 & 188 & 198 & 154 & 204 & 214 & 212 & 146 & 136 & 86\end{array}$

070437A $2000 \quad 178 \quad 186 \quad 82-9999$

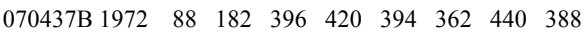

$\begin{array}{lllllllllll}070437 \mathrm{~B} 1980 & 702 & 450 & 284 & 382 & 302 & 332 & 250 & 206 & 160 & 228\end{array}$

070437B 1990 $178 \begin{array}{llllllllll}178 & 258 & 212 & 230 & 232 & 174 & 212 & 130 & 60\end{array}$

070437B $2000 \quad 96 \quad 94 \quad 44-9999$

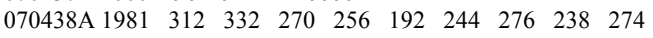

$\begin{array}{lllllllllll}070438 \text { A } 1990 & 160 & 130 & 148 & 108 & 96 & 122 & 80 & 66 & 70 & 38\end{array}$

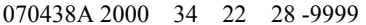

070438B $1978 \quad 276 \quad 332$

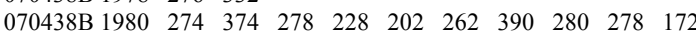

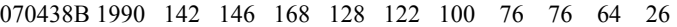

070438B $2000 \quad 44 \quad 28 \quad 26-9999$

$\begin{array}{llllllllll}070439 \text { A } 1971 & 302 & 226 & 314 & 378 & 430 & 324 & 344 & 484 & 490\end{array}$

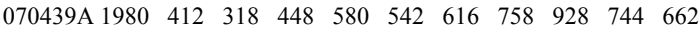

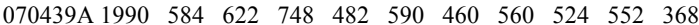
070439A $2000 \quad 432 \quad 374 \quad 270-9999$

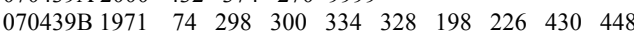

070439B 1980 $46 \begin{array}{llllllllll}258 & 280 & 422 & 362 & 400 & 420 & 416 & 432 & 464\end{array}$

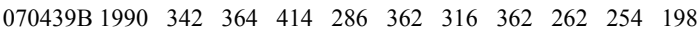

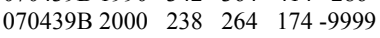


070440A $1975 \quad 198 \quad 190 \quad 306 \quad 116 \quad 186$

070440A 1980 $198 \begin{array}{llllllllll}410 & 278 & 366 & 410 & 324 & 272 & 286 & 332 & 232\end{array}$

$\begin{array}{llllllllllll}070440 \text { A } 1990 & 192 & 120 & 176 & 286 & 132 & 62 & 116 & 172 & 102 & 98\end{array}$

070440A $2000 \quad 120 \quad 128 \quad 92-9999$

070440B $1977 \quad 422 \quad 278 \quad 372$

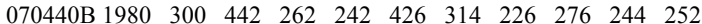

$\begin{array}{lllllllllll}070440 \mathrm{~B} 1990 & 208 & 338 & 208 & 170 & 164 & 336 & 182 & 272 & 112 & 120\end{array}$

070440B 2000 $318 \quad 204 \quad 146-9999$

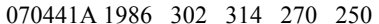

$\begin{array}{lllllllllll}070441 \mathrm{~A} 1990 & 264 & 172 & 208 & 272 & 158 & 292 & 206 & 128 & 166 & 136\end{array}$

070441A $2000 \quad 98 \quad 62 \quad 48-9999$

070441B $1986 \quad 406 \quad 286 \quad 286 \quad 216$

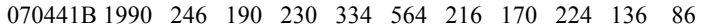

070441B $2000 \quad 62 \quad 54 \quad 22-9999$

$\begin{array}{llllllllll}070446 \text { A } 1981 & 102 & 214 & 156 & 176 & 168 & 136 & 140 & 152 & 108\end{array}$

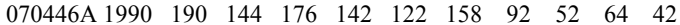

070446A $2000 \quad 32 \quad 42 \quad 26-9999$

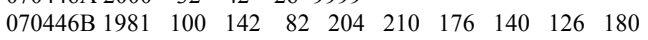

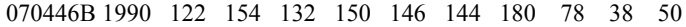

070446B 2000 $38 \quad 24 \quad 66-9999$

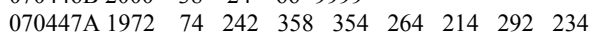

$\begin{array}{lllllllllll}070447 \mathrm{~A} 1980 & 354 & 258 & 258 & 290 & 262 & 218 & 182 & 170 & 136 & 136\end{array}$

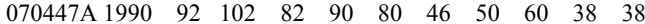

070447A $2000 \quad 18 \quad 26 \quad 34-9999$

$\begin{array}{llllllll}070447 \mathrm{~B} 1973 & 230 & 276 & 278 & 334 & 330 & 268 & 202\end{array}$

$\begin{array}{lllllllllll}070447 \mathrm{~B} 1980 & 308 & 234 & 424 & 300 & 292 & 246 & 206 & 198 & 208 & 216\end{array}$

$\begin{array}{lllllllllll}070447 \mathrm{~B} 1990 & 130 & 90 & 196 & 138 & 142 & 172 & 130 & 118 & 74 & 68\end{array}$

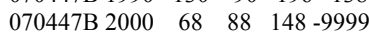

070448A $1977 \quad 540 \quad 434 \quad 396$

070448A 1980 $346 \begin{array}{lllllllll}342 & 424 & 478 & 480 & 502 & 442 & 350 & 444 & 346\end{array}$ $\begin{array}{lllllllllll}070448 \text { A } 1990 & 386 & 350 & 262 & 222 & 346 & 156 & 300 & 196 & 360 & 192\end{array}$ 070448A $2000 \quad 112 \quad 210 \quad 116-9999$

$\begin{array}{llllllll}070448 \text { B } 1973 & 246 & 300 & 582 & 352 & 322 & 308 & 404\end{array}$

070448B 1980 $394 \begin{array}{lllllllll}604 & 466 & 546 & 582 & 432 & 382 & 300 & 308 & 200\end{array}$ $\begin{array}{lllllllllllll}070448 B & 1990 & 320 & 304 & 214 & 378 & 226 & 294 & 222 & 456 & 194 & 120\end{array}$ 070448B $2000 \quad 90 \quad 180 \quad 120-9999$

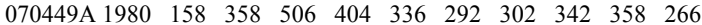
$\begin{array}{llllllllllll}070449 \text { A } 1990 & 318 & 370 & 218 & 216 & 186 & 208 & 68 & 136 & 68 & 74\end{array}$ 070449A $2000 \quad 80 \quad 72 \quad 24-9999$

070450A $1973 \quad 332 \quad 394 \quad 544 \quad 440 \quad 322 \quad 260 \quad 324$

$\begin{array}{lllllllllll}070450 \mathrm{~A} 1980 & 318 & 492 & 374 & 472 & 510 & 330 & 346 & 370 & 230 & 206\end{array}$ $\begin{array}{llllllllllll}070450 \mathrm{~A} 1990 & 308 & 222 & 106 & 230 & 158 & 234 & 132 & 236 & 228 & 370\end{array}$ 070450A $2000 \quad 178 \quad 38 \quad 174-9999$

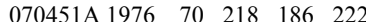

$\begin{array}{llllllllllll}070451 \mathrm{~A} & 1980 & 294 & 192 & 116 & 134 & 108 & 88 & 114 & 108 & 66 & 40\end{array}$

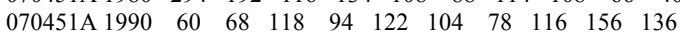
070451A $2000 \quad 100 \quad 146 \quad 126-9999$

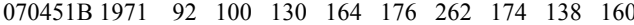

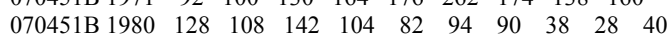

$\begin{array}{lllllllllll}070451 \mathrm{~B} 1990 & 32 & 28 & 54 & 58 & 74 & 76 & 54 & 96 & 138 & 116\end{array}$

070451B $2000 \quad 88 \quad 136 \quad 100-9999$

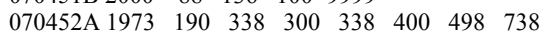

$\begin{array}{lllllllllll}070452 \mathrm{~A} 1980 & 394 & 528 & 482 & 384 & 396 & 428 & 352 & 150 & 114 & 352\end{array}$

$\begin{array}{llllllllllll}070452 \mathrm{~A} & 1990 & 284 & 238 & 452 & 374 & 438 & 402 & 406 & 356 & 374 & 212\end{array}$

$\begin{array}{lllll}070452 \text { A } 2000 & 274 & 222 & 132 & -9999\end{array}$

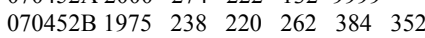

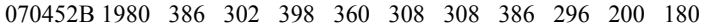

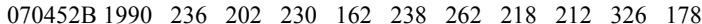

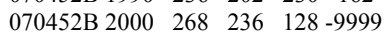

070454A $1974 \quad 334 \quad 1036 \quad 678 \quad 672 \quad 646 \quad 538$

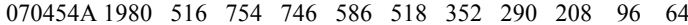

$\begin{array}{lllllllllll}070454 \mathrm{~A} 1990 & 222 & 236 & 174 & 278 & 198 & 156 & 100 & 106 & 148 & 118\end{array}$ 070454A $2000 \quad 78 \quad 90 \quad 88-9999$

070454B $1975 \quad 1088 \quad 622 \quad 520 \quad 594 \quad 696$

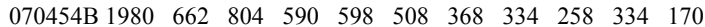
$\begin{array}{lllllllllll}\text { 070454B } 1990 & 102 & 308 & 244 & 180 & 246 & 180 & 256 & 96 & 94 & 134\end{array}$ 070454B $2000 \quad 64 \quad 44 \quad 134-9999$

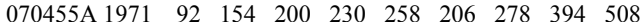

$\begin{array}{llllllllllll}070455 \mathrm{~A} & 1980 & 676 & 536 & 486 & 508 & 454 & 528 & 580 & 682 & 600 & 578\end{array}$

$\begin{array}{llllllllllll}070455 \mathrm{~A} 1990 & 670 & 542 & 890 & 556 & 532 & 374 & 336 & 394 & 438 & 222\end{array}$

$\begin{array}{llllll}070455 \mathrm{~A} & 2000 & 384 & 270 & 238 & -9999\end{array}$

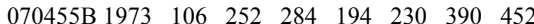

$\begin{array}{lllllllllll}070455 \mathrm{~B} 1980 & 594 & 292 & 298 & 324 & 276 & 340 & 406 & 332 & 290 & 370\end{array}$

$\begin{array}{llllllllllll}070455 \mathrm{~B} & 1990 & 362 & 242 & 260 & 204 & 316 & 248 & 408 & 306 & 430 & 166\end{array}$

070455B $2000 \quad 262 \quad 286 \quad 162-9999$

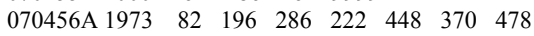

$\begin{array}{lllllllllll}070456 \text { A } 1980 & 494 & 584 & 550 & 378 & 358 & 384 & 406 & 264 & 216 & 296\end{array}$

$\begin{array}{lllllllllll}070456 \text { A } 1990 & 340 & 268 & 438 & 210 & 190 & 198 & 172 & 206 & 272 & 192\end{array}$

070456A $2000 \quad 160 \quad 176 \quad 156-9999$

070456B $1975 \quad 66 \quad 212 \quad 488 \quad 500 \quad 502$

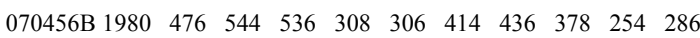
070456B 1990 $26 \begin{array}{llllllllll}160 & 254 & 152 & 160 & 266 & 98 & 186 & 258 & 162\end{array}$ 070456B $2000 \quad 188 \quad 170 \quad 120-9999$

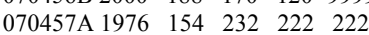

$\begin{array}{llllllllllll}070457 \text { A } 1980 & 156 & 248 & 392 & 408 & 356 & 296 & 306 & 268 & 266 & 188\end{array}$

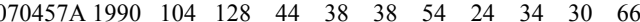

070457A $2000 \quad 32 \quad 12 \quad 14-9999$

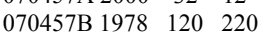

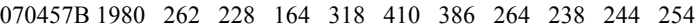

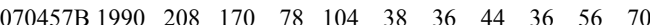

$\begin{array}{lllll}070457 \mathrm{~B} 2000 & 134 & 52 & 32 & -9999\end{array}$

070458A $1974 \quad 404 \quad 272 \quad 352 \quad 402 \quad 328 \quad 178$

$\begin{array}{lllllllllll}070458 \text { A } 1980 & 32 & 58 & 22 & 14 & 22 & 34 & 16 & 18 & 40 & 48\end{array}$

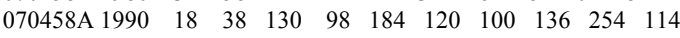

$\begin{array}{lllll}070458 \text { A } 2000 & 244 & 238 & 136 & -9999\end{array}$

070458B $1978 \quad 272 \quad 308$

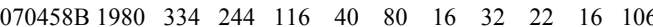

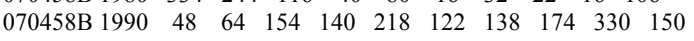

070458B $2000 \quad 246 \quad 240 \quad 166-9999$

070459A $1987 \quad 102 \quad 254 \quad 222$

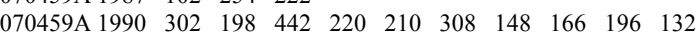
070459A $2000 \quad 80 \quad 126 \quad 42-9999$

070460A $1977 \quad 282 \quad 384 \quad 556$

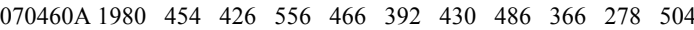

$\begin{array}{llllllllllll}070460 \text { A } 1990 & 550 & 370 & 348 & 244 & 302 & 314 & 324 & 268 & 300 & 228\end{array}$

070460A $2000 \quad 486 \quad 340 \quad 222-9999$

070462A $1973 \quad 122 \quad 296 \quad 532 \quad 454 \quad 620 \quad 616 \quad 440$

$\begin{array}{lllllllllll}070462 \mathrm{~A} 1981 & 580 & 552 & 654 & 388 & 426 & 474 & 570 & 390 & 346 & 500\end{array}$

$\begin{array}{lllllllllll}070462 \text { A } 1990 & 396 & 284 & 318 & 312 & 432 & 372 & 258 & 250 & 282 & 148\end{array}$

070462A $2000 \quad 210 \quad 162 \quad 182-9999$

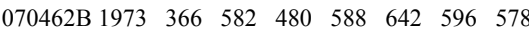

$\begin{array}{llllllllllll}070462 \mathrm{~B} & 1980 & 486 & 494 & 642 & 638 & 698 & 644 & 420 & 398 & 668 & 630\end{array}$

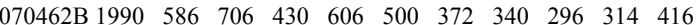

070462 B $2000 \quad 246 \quad 130 \quad 90-9999$

070501 A 1979232

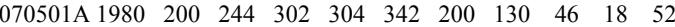

$\begin{array}{lllllllllll}070501 \mathrm{~A} 1990 & 16 & 14 & 22 & 4 & 14 & 84 & 102 & 90 & 56 & 16\end{array}$

070501A $2000 \quad 12 \quad 32 \quad 48-9999$

$\begin{array}{lllllllllll}070501 \mathrm{~B} 1980 & 196 & 230 & 214 & 226 & 260 & 258 & 288 & 258 & 164 & 48\end{array}$

$\begin{array}{lllllllllll}070501 \mathrm{~B} 1990 & 16 & 14 & 4 & 30 & 82 & 66 & 78 & 22 & 8 & 10\end{array}$

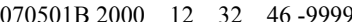

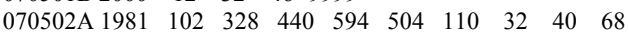

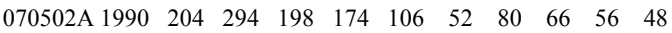

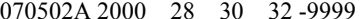

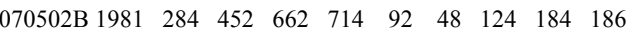

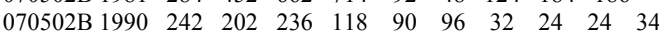

070502B $2000 \quad 14 \quad 22 \quad 36-9999$

070504A $1988 \quad 460 \quad 202$

$\begin{array}{lllllllllll}070504 \text { A } 1990 & 162 & 148 & 168 & 188 & 246 & 170 & 376 & 228 & 180 & 108\end{array}$ 070504A $2000 \quad 172 \quad 98 \quad 194-9999$

070504B $1988 \quad 276 \quad 192$

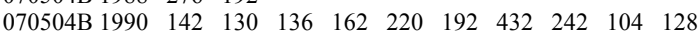

070504B 2000 $284 \quad 98 \quad 104-9999$

070505A $1977 \quad 268 \quad 476 \quad 696$

$\begin{array}{llllllllllll}070505 \text { A } 1980 & 634 & 654 & 558 & 528 & 442 & 488 & 594 & 492 & 410 & 232\end{array}$

$\begin{array}{lllllllllll}070505 \text { A } 1990 & 294 & 478 & 282 & 272 & 328 & 340 & 318 & 270 & 344 & 202\end{array}$

070505A $2000 \quad 344 \quad 394 \quad 178-9999$

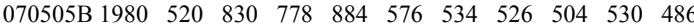

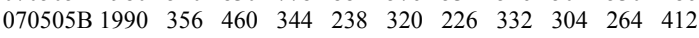

070505B 2000 $356 \quad 322 \quad 148-9999$

070506A 1979108

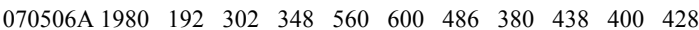

$\begin{array}{lllllllllll}070506 \text { A } 1990 & 360 & 204 & 104 & 106 & 58 & 80 & 56 & 26 & 24 & 16\end{array}$

070506A $2000 \quad 18 \quad 20 \quad 30-9999$

070506B 1980 $94 \begin{array}{llllllllll}202 & 290 & 378 & 628 & 610 & 192 & 140 & 424 & 554\end{array}$

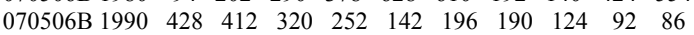

070506B $2000 \quad 22 \quad 46 \quad 34-9999$

070507A $1976 \quad 192 \quad 562 \quad 518 \quad 468$

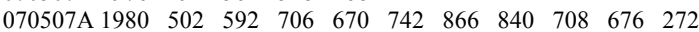

$\begin{array}{lllllllllll}070507 \mathrm{~A} 1990 & 174 & 190 & 158 & 352 & 194 & 148 & 168 & 116 & 94 & 126\end{array}$

$\begin{array}{lllll}070507 \mathrm{~A} 2000 & 58 & 152 & 172 & -9999\end{array}$

$\begin{array}{llllllllll}070507 \mathrm{~B} 1981 & 658 & 602 & 666 & 618 & 664 & 862 & 810 & 644 & 700\end{array}$

$\begin{array}{llllllllllll}070507 \mathrm{~B} & 1990 & 712 & 650 & 652 & 358 & 356 & 292 & 282 & 274 & 294 & 530\end{array}$

070507B $2000 \quad 218 \quad 316 \quad 394-9999$

070508A $1977 \quad 402 \quad 754 \quad 640$

070508A $19801376 \quad 906 \quad 938 \quad 1060 \quad 794 \quad 1036 \quad 898 \quad 774 \quad 300 \quad 604$

$\begin{array}{lllllllllll}070508 \text { A } 1990 & 870 & 554 & 618 & 452 & 440 & 620 & 570 & 558 & 556 & 268\end{array}$

070508A $2000 \quad 408 \quad 566 \quad 266-9999$

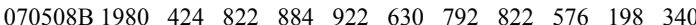

$\begin{array}{lllllllllll}070508 \mathrm{~B} 1990 & 384 & 308 & 400 & 240 & 248 & 200 & 206 & 178 & 202 & 120\end{array}$ 
070508B $2000 \quad 170 \quad 362 \quad 82-9999$

070509A $1975 \quad 180 \quad 338 \quad 358 \quad 476 \quad 400$

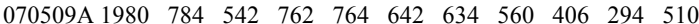

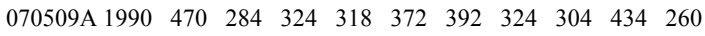

070509A $2000 \quad 450 \quad 574 \quad 248-9999$

070509B $1975 \quad 282 \quad 266 \quad 278 \quad 456 \quad 502$

$\begin{array}{lllllllllll}070509 \mathrm{~B} 1980 & 642 & 502 & 538 & 650 & 590 & 632 & 710 & 638 & 454 & 444\end{array}$

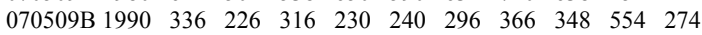

070509B $2000 \quad 338 \quad 382 \quad 100-9999$

070510A $1984 \quad 162 \quad 426 \quad 890 \quad 598 \quad 490 \quad 292$

$\begin{array}{llllllllllll}070510 A & 1990 & 386 & 296 & 336 & 288 & 258 & 184 & 150 & 170 & 90 & 180\end{array}$

070510A $2000 \quad 126 \quad 78 \quad 114-9999$

070510B $1984 \quad 642 \quad 454 \quad 238 \quad 252 \quad 340 \quad 402$

$\begin{array}{llllllllllll}070510 \mathrm{~B} 1990 & 424 & 408 & 528 & 482 & 406 & 274 & 168 & 148 & 270 & 92\end{array}$

070510B $2000 \quad 148 \quad 142 \quad 86-9999$

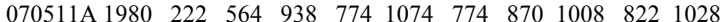

070511A $19901090 \quad 834 \quad 476 \quad 580 \quad 776 \quad 1456 \quad 844 \quad 406 \quad 502 \quad 976$

070511A $2000 \quad 620 \quad 316 \quad 336-9999$

070511B 1979190

070511B $19802058 \quad 1108 \quad 1204 \quad 1266 \quad 1050 \quad 1198 \quad 1134 \quad 668 \quad 184 \quad 210$

$\begin{array}{lllllllllll}070511 \mathrm{~B} 1990 & 382 & 224 & 346 & 310 & 360 & 504 & 464 & 478 & 666 & 280\end{array}$

070511B $2000 \quad 480 \quad 616 \quad 218-9999$

$\begin{array}{lllllllllll}070512 \text { A } 1981 & 272 & 362 & 288 & 168 & 472 & 446 & 510 & 818 & 220\end{array}$

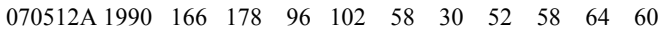

070512A $2000 \quad 44 \quad 94 \quad 86-9999$

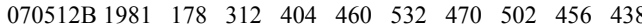

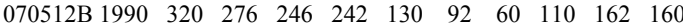

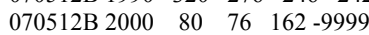

070513A 1979400

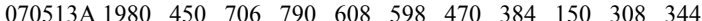

$\begin{array}{lllllllllll}070513 \text { A } 1990 & 196 & 164 & 128 & 188 & 118 & 68 & 42 & 12 & 16 & 8\end{array}$

$\begin{array}{lllll}070513 \text { A } 2000 & 12 & 10 & 26 & -9999\end{array}$

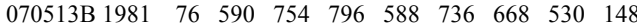

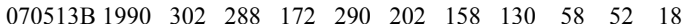

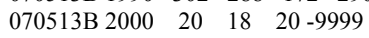

070514A $1996 \quad 308 \quad 292 \quad 168 \quad 262$

070514A $2000 \quad 256 \quad 182 \quad 192 \quad-9999$

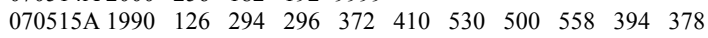

070515A $2000 \quad 246 \quad 256 \quad 150-9999$

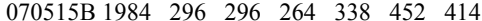

$\begin{array}{llllllllllll}070515 \mathrm{~B} 1990 & 388 & 340 & 258 & 202 & 112 & 122 & 118 & 72 & 48 & 70\end{array}$

$\begin{array}{lcccc}070515 \mathrm{~B} 2000 & 68 & 52 & 122 & -9999\end{array}$

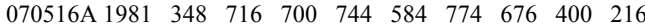

$\begin{array}{lllllllllll}070516 \text { A } 1990 & 302 & 352 & 168 & 334 & 208 & 376 & 334 & 324 & 322 & 334\end{array}$

$\begin{array}{llllll}070516 \text { A } 2000 & 358 & 342 & 132 & -9999\end{array}$

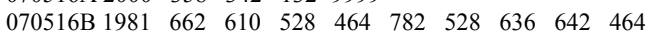

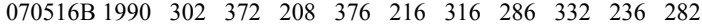

070516B $2000 \quad 310 \quad 334 \quad 140-9999$

070517A $1976 \quad 286 \quad 494 \quad 490 \quad 660$

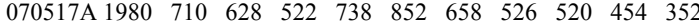

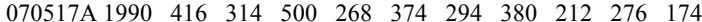

070517A $2000 \quad 244 \quad 464 \quad 124-9999$

070517B $1978 \quad 696 \quad 816$

$\begin{array}{lllllllllll}070517 \mathrm{~B} 1980 & 634 & 934 & 1342 & 662 & 760 & 794 & 668 & 630 & 1506 & 418\end{array}$

$\begin{array}{llllllllllll}070517 \mathrm{~B} 1990 & 562 & 392 & 470 & 454 & 710 & 382 & 528 & 250 & 38 & 38\end{array}$

$\begin{array}{lllll}070517 \mathrm{~B} 2000 & 28 & 22 & 140 & -9999\end{array}$

$\begin{array}{llllll}070518 A & 1976 & 710 & 378 & 392 & 372\end{array}$

$\begin{array}{lllllllllll}070518 \text { A } 1980 & 444 & 494 & 504 & 400 & 502 & 352 & 248 & 440 & 328 & 282\end{array}$

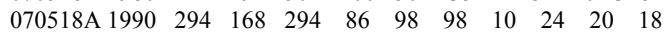

$\begin{array}{lllll}070518 \text { A } 2000 & 24 & 52 & 22 & -9999\end{array}$

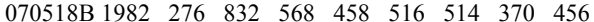

$\begin{array}{lllllllllll}070518 \mathrm{~B} 1990 & 530 & 430 & 344 & 330 & 208 & 228 & 220 & 150 & 110 & 112\end{array}$

070518B $2000 \quad 142 \quad 70 \quad 100-9999$

070519A $1975 \quad 198 \quad 208 \quad 308 \quad 328 \quad 292$

$\begin{array}{lllllllllll}070519 \text { A } 1980 & 380 & 332 & 298 & 342 & 282 & 296 & 162 & 182 & 202 & 198\end{array}$ $\begin{array}{lllllllllll}\text { 070519A } 1990 & 142 & 262 & 224 & 160 & 284 & 208 & 184 & 184 & 86 & 102\end{array}$ 070519A $2000 \quad 80 \quad 64 \quad 62-9999$

070519B $1979 \quad 190$

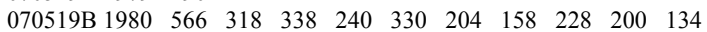

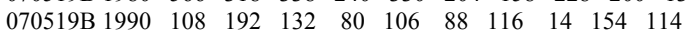

070519B 2000 $118 \quad 66 \quad 48-9999$

070520A $1977 \quad 554 \quad 330 \quad 512$

$\begin{array}{lllllllllll}070520 \mathrm{~A} 1980 & 470 & 540 & 438 & 556 & 686 & 332 & 238 & 264 & 234 & 240\end{array}$

$\begin{array}{lllllllllll}070520 \text { A } 1990 & 262 & 286 & 248 & 408 & 220 & 218 & 122 & 204 & 204 & 130\end{array}$

070520A $2000 \quad 114 \quad 74 \quad 172-9999$

070520B $1975 \quad 248 \quad 300 \quad 322 \quad 428 \quad 480$

$\begin{array}{lllllllllll}070520 \mathrm{~B} 1980 & 516 & 474 & 676 & 718 & 482 & 350 & 382 & 408 & 318 & 268\end{array}$

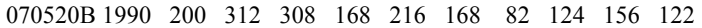

070520B $2000 \quad 184 \quad 116 \quad 72-9999$

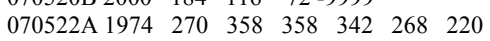

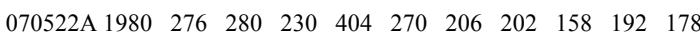
$\begin{array}{llllllllllll}070522 \mathrm{~A} & 1990 & 130 & 116 & 102 & 100 & 94 & 106 & 100 & 68 & 76 & 44\end{array}$

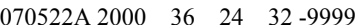

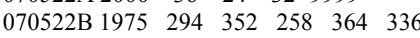

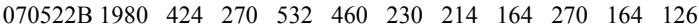

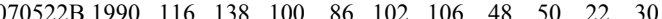

070522B $2000 \quad 36 \quad 32 \quad 32-9999$

070523A $1994 \quad 420 \quad 346 \quad 256 \quad 232 \quad 228 \quad 194$

070523A $2000 \quad 518 \quad 164 \quad 136-9999$

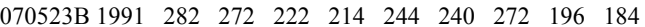

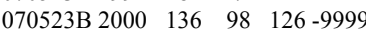

070524A $1988 \quad 234 \quad 168$

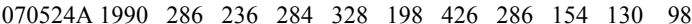

070524A $2000 \quad 92 \quad 90 \quad 92-9999$

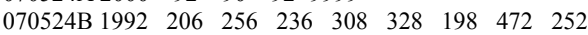

070524B $2000 \quad 162 \quad 240 \quad 140-9999$

070525A 1989680

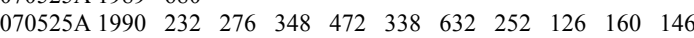
070525A $2000 \quad 62 \quad 40 \quad 34-9999$

$\begin{array}{lllllllllll}070526 \text { A } 1980 & 322 & 440 & 454 & 574 & 592 & 494 & 358 & 296 & 170 & 182\end{array}$ $\begin{array}{lllllllllll}070526 \text { A } 1990 & 220 & 196 & 222 & 190 & 186 & 180 & 136 & 164 & 140 & 192\end{array}$ 070526A $2000 \quad 148 \quad 204 \quad 98-9999$

$070526 \mathrm{~B} 1976 \quad 72 \quad 462 \quad 532 \quad 666$

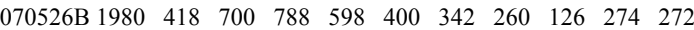

$\begin{array}{llllllllllll}070526 \mathrm{~B} & 1990 & 310 & 210 & 254 & 238 & 50 & 76 & 92 & 172 & 90 & 210\end{array}$

070526B 2000 $106 \quad 114 \quad 86-9999$

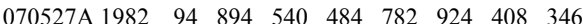

$\begin{array}{lllllllllll}070527 \mathrm{~A} 1990 & 208 & 292 & 200 & 222 & 286 & 242 & 296 & 174 & 230 & 172\end{array}$ 070527A $2000 \quad 226 \quad 348 \quad 148-9999$

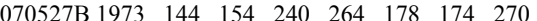

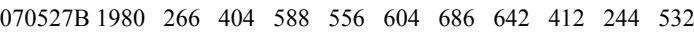

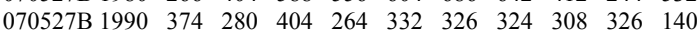

070527B $2000 \quad 246 \quad 308 \quad 138-9999$

$\begin{array}{llllll}070528 A & 1976 & 230 & 252 & 100 & 222\end{array}$

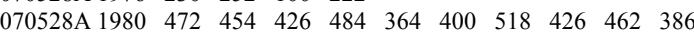

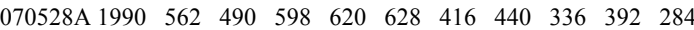
070528 A $2000 \quad 264 \quad 148 \quad 110-9999$

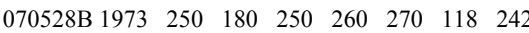

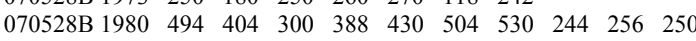

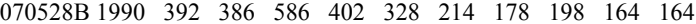
070528B $2000 \quad 204 \quad 140 \quad 68-9999$

$\begin{array}{llllllllll}070529 \text { A } 1991 & 408 & 348 & 308 & 332 & 262 & 182 & 154 & 290 & 142\end{array}$

070529A $2000 \quad 162 \quad 192 \quad 110-9999$

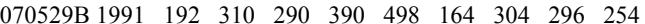

070529B 2000 $254 \quad 246 \quad 272-9999$

070530A 1979232

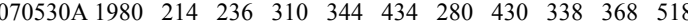
$\begin{array}{llllllllllll}070530 \mathrm{~A} 1990 & 468 & 422 & 248 & 196 & 116 & 118 & 176 & 118 & 138 & 38\end{array}$ $\begin{array}{lllll}070530 \text { A } 2000 \quad 82 & 32 & 30 & -9999\end{array}$

070530B $1978 \quad 174 \quad 496$

070530B 1980 $640 \begin{array}{lllllllll}446 & 446 & 478 & 428 & 532 & 486 & 336 & 172 & 114\end{array}$ $\begin{array}{lllllllllll}070530 \mathrm{~B} 1990 & 230 & 130 & 148 & 166 & 106 & 58 & 18 & 16 & 14 & 24\end{array}$ 070530B $2000 \quad 20 \quad 14 \quad 30-9999$

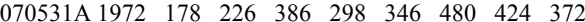

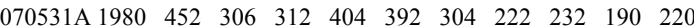

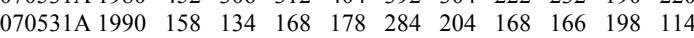
070531A $2000 \quad 204 \quad 240 \quad 96-9999$

070531 B $1978 \quad 218 \quad 290$

$\begin{array}{lllllllllll}070531 \mathrm{~B} 1980 & 422 & 398 & 454 & 404 & 818 & 420 & 604 & 508 & 540 & 514\end{array}$

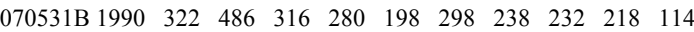
070531B $2000 \quad 264 \quad 152 \quad 180-9999$

$\begin{array}{lllllllllll}070532 \mathrm{~A} 1980 & 156 & 304 & 358 & 288 & 450 & 332 & 230 & 354 & 264 & 260\end{array}$

$\begin{array}{llllllllllll}070532 \text { A } 1990 & 196 & 132 & 162 & 216 & 224 & 134 & 270 & 278 & 174 & 158\end{array}$ 070532A $2000 \quad 78 \quad 160 \quad 80-9999$

070532B $1982 \quad 326 \quad 312 \quad 312 \quad 406 \quad 264 \quad 222 \quad 350 \quad 256$

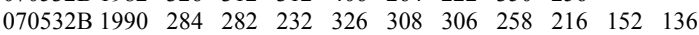

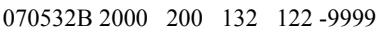

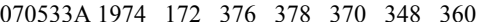

$\begin{array}{llllllllllll}070533 \text { A } 1980 & 320 & 320 & 230 & 258 & 268 & 216 & 310 & 282 & 220 & 140\end{array}$

$\begin{array}{lllllllllllll}070533 \mathrm{~A} & 1990 & 184 & 224 & 226 & 282 & 202 & 170 & 170 & 258 & 54 & 80\end{array}$

070533A $2000 \quad 86 \quad 44 \quad 66-9999$

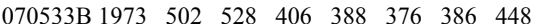

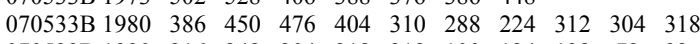

$\begin{array}{lllllllllll}070533 \mathrm{~B} 1990 & 216 & 248 & 204 & 218 & 218 & 100 & 124 & 138 & 72 & 92\end{array}$

070533B $2000 \quad 116 \quad 114 \quad 86-9999$

070534A $1984 \quad 328 \quad 314 \quad 392 \quad 316 \quad 218 \quad 376$

$\begin{array}{lllllllllll}070534 \mathrm{~A} 1990 & 222 & 52 & 56 & 54 & 84 & 46 & 34 & 38 & 48 & 34\end{array}$

070534A 2000 $72 \quad 42 \quad 54-9999$

070534B $1977 \quad 116 \quad 82 \quad 62$

$\begin{array}{lllllllllll}070534 \mathrm{~B} 1980 & 182 & 318 & 426 & 422 & 286 & 344 & 350 & 216 & 206 & 180\end{array}$ 


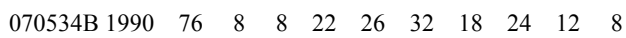
070534B 2000 $\quad 8 \quad 4 \quad 8$-9999

070535 A $1978 \quad 358 \quad 680$

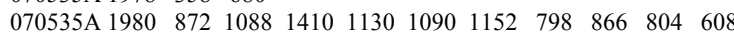

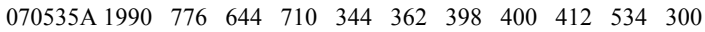
070535 A $2000 \quad 444 \quad 562 \quad 226-9999$

070535B 1979244

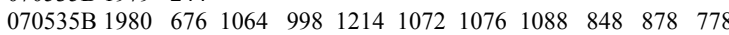

$\begin{array}{llllllllllll}070535 \mathrm{~B} 1990 & 772 & 294 & 428 & 230 & 388 & 334 & 366 & 458 & 712 & 326\end{array}$

070535B $2000 \quad 538 \quad 476 \quad 168-9999$

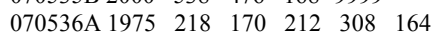

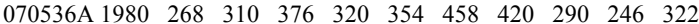

$\begin{array}{llllllllllll}070536 \text { A } 1990 & 246 & 130 & 152 & 212 & 214 & 152 & 100 & 66 & 76 & 66\end{array}$

070536A $2000 \quad 78 \quad 98 \quad 98-9999$

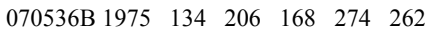

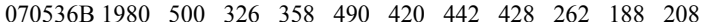

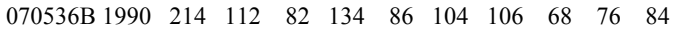

070536B $2000 \quad 80 \quad 84 \quad 44-9999$

070537A $1979 \quad 88$

070537A $1980 \quad 368 \quad 402 \quad 582 \quad 476 \quad 566 \quad 660 \quad 666 \quad 766 \quad 734 \quad 502$

$\begin{array}{lllllllllll}070537 \mathrm{~A} 1990 & 594 & 304 & 202 & 278 & 246 & 464 & 540 & 408 & 288 & 394\end{array}$

070537A $2000 \quad 602 \quad 506 \quad 182-9999$

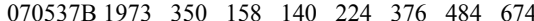

$\begin{array}{llllllllllll}070537 \mathrm{~B} 1980 & 508 & 598 & 614 & 560 & 688 & 636 & 172 & 186 & 174 & 190\end{array}$

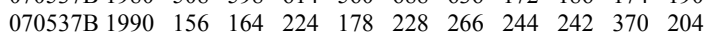

070537B $2000 \quad 398 \quad 478 \quad 138-9999$

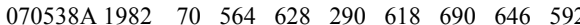

$\begin{array}{llllllllllll}070538 \text { A } 1990 & 696 & 452 & 502 & 1122 & 328 & 148 & 78 & 90 & 72 & 42\end{array}$

$\begin{array}{lllll}070538 \text { A } 2000 & 38 & 52 & 26 & -9999\end{array}$

070538B $1974 \quad 186 \quad 280 \quad 222 \quad 294 \quad 376 \quad 388$

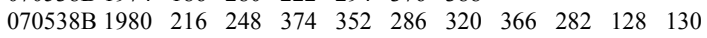

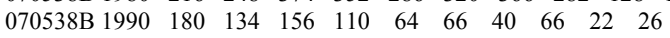

070538B 2000 $46 \quad 70 \quad 32-9999$

070539A $1974 \quad 162 \quad 154 \quad 142 \quad 140 \quad 218 \quad 178$

$\begin{array}{lllllllllll}070539 \text { A } 1980 & 178 & 156 & 62 & 52 & 64 & 42 & 52 & 94 & 70 & 66\end{array}$

$\begin{array}{lllllllllll}\text { 070539A } 1990 & 92 & 88 & 192 & 112 & 122 & 82 & 36 & 34 & 18 & 58\end{array}$

070539A $2000 \quad 58 \quad 30 \quad 20-9999$

070539B $1976 \quad 180 \quad 136 \quad 198 \quad 114$

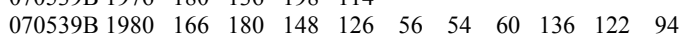

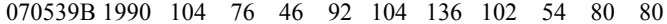

070539B 2000 $34 \quad 10 \quad 20-9999$

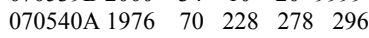

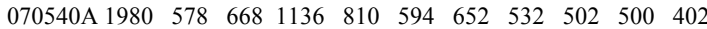

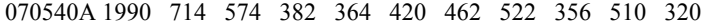

070540A $2000 \quad 592 \quad 686 \quad 238-9999$

070540B $1976 \quad 348 \quad 328 \quad 510 \quad 576$

070540B 1980 $496 \begin{array}{llllllllll}660 & 580 & 424 & 374 & 406 & 488 & 318 & 238 & 290\end{array}$

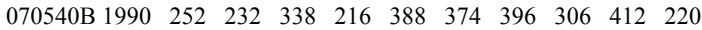

070540B 2000 $406 \quad 486 \quad 186-9999$

070541A $1975 \quad 396 \quad 1242 \quad 1050 \quad 772 \quad 1152$

$\begin{array}{llllllllllll}070541 \mathrm{~A} & 1980 & 1142 & 890 & 1030 & 888 & 956 & 966 & 628 & 490 & 588 & 662\end{array}$

$\begin{array}{lllllllllll}070541 \mathrm{~A} 1990 & 494 & 504 & 314 & 318 & 306 & 222 & 182 & 82 & 98 & 100\end{array}$

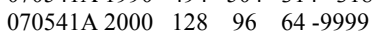

070541B $1973 \quad 192 \quad 402 \quad 460 \quad 506 \quad 748 \quad 766 \quad 604$

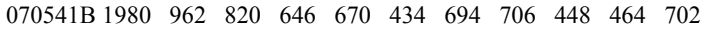

$\begin{array}{lllllllllll}070541 \mathrm{~B} 1990 & 740 & 626 & 630 & 472 & 388 & 386 & 276 & 198 & 152 & 158\end{array}$

070541B $2000 \quad 140 \quad 104 \quad 82-9999$

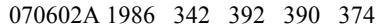

$\begin{array}{lllllllllll}070602 \text { A } 1990 & 378 & 292 & 178 & 138 & 128 & 138 & 194 & 68 & 34 & 32\end{array}$

070602A $2000 \quad 68 \quad 104 \quad 48-9999$

070602B $1975 \quad 248 \quad 404 \quad 474 \quad 340 \quad 258$

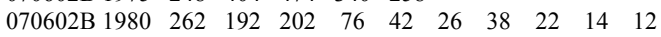

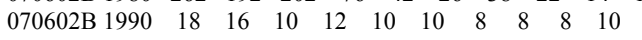

070602B $2000 \quad 6 \quad 6 \quad 6-9999$

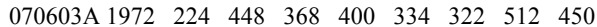

$\begin{array}{llllllllllll}070603 \text { A } 1980 & 528 & 678 & 772 & 600 & 722 & 752 & 676 & 458 & 354 & 460\end{array}$

$\begin{array}{lllllllllll}070603 \text { A } 1990 & 542 & 496 & 502 & 392 & 422 & 482 & 400 & 228 & 192 & 244\end{array}$

$\begin{array}{lllll}070603 \text { A } 2000 & 288 & 324 & 240 & -9999\end{array}$

070603B 1979124

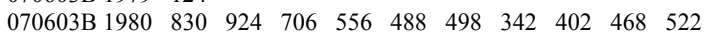

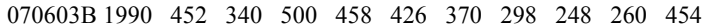

070603B $2000 \quad 298 \quad 288 \quad 342-9999$

070604A $1977 \quad 72 \quad 402 \quad 484$

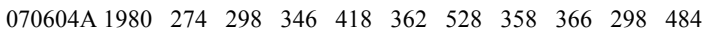

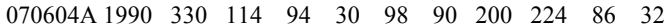

070604A $2000 \quad 68 \quad 46 \quad 28-9999$

070604B $1978 \quad 160 \quad 472$

070604B 1980 $330 \begin{array}{llllllllll}300 & 320 & 326 & 408 & 170 & 270 & 270 & 308 & 218\end{array}$

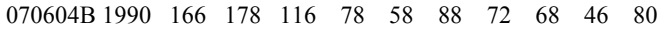

070604B $2000 \quad 24 \quad 120 \quad 178-9999$
070605A $1976 \quad 106 \quad 454 \quad 502 \quad 548$

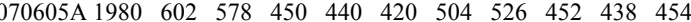

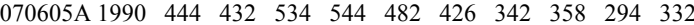

$\begin{array}{lllll}070605 \text { A } 2000 & 334 & 316 & 178 & -9999\end{array}$

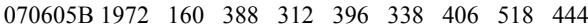

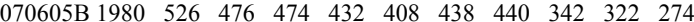

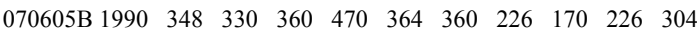

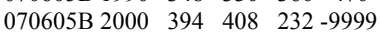

070606A $1974 \quad 150 \quad 292 \quad 274 \quad 272 \quad 454 \quad 388$

$\begin{array}{lllllllllll}070606 \text { A } 1980 & 492 & 412 & 332 & 230 & 200 & 184 & 274 & 202 & 184 & 182\end{array}$

$\begin{array}{lllllllllll}\text { 070606A } 1990 & 140 & 88 & 62 & 60 & 52 & 62 & 52 & 88 & 128 & 80\end{array}$

070606A $2000 \quad 122 \quad 82 \quad 84-9999$

070606B $1971 \quad 268 \quad 270 \begin{array}{llllllll}230 & 222 & 220 & 266 & 378 & 466 & 394\end{array}$

$\begin{array}{llllllllllll}070606 \mathrm{~B} & 1980 & 428 & 488 & 404 & 310 & 260 & 240 & 300 & 222 & 164 & 194\end{array}$

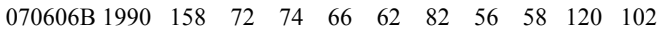

070606B $2000 \quad 104 \quad 94 \quad 70-9999$

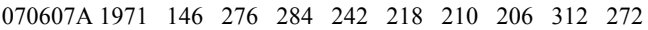

$\begin{array}{llllllllllll}070607 \text { A } 1980 & 278 & 302 & 202 & 230 & 190 & 190 & 164 & 166 & 112 & 148\end{array}$

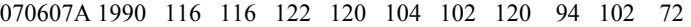

070607A $2000 \quad 50 \quad 48 \quad 52-9999$

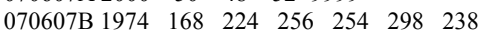

$\begin{array}{lllllllllllll}070607 \mathrm{~B} & 1980 & 234 & 308 & 314 & 274 & 234 & 246 & 202 & 190 & 102 & 188\end{array}$

$\begin{array}{lllllllllll}070607 \mathrm{~B} 1990 & 138 & 110 & 120 & 120 & 102 & 112 & 100 & 92 & 110 & 74\end{array}$

070607B $2000 \quad 48 \quad 46 \quad 48-9999$

$\begin{array}{lllllllll}070608 \text { A } 1973 & 116 & 162 & 158 & 150 & 182 & 186 & 90\end{array}$

$\begin{array}{lllllllllll}070608 \text { A } 1980 & 66 & 74 & 48 & 22 & 20 & 46 & 40 & 32 & 32 & 38\end{array}$

$\begin{array}{lllllllllll}070608 \text { A } 1990 & 30 & 24 & 66 & 80 & 44 & 26 & 24 & 38 & 46 & 38\end{array}$

$\begin{array}{lllll}070608 \mathrm{~A} & 2000 & 30 & 38 & 30-9999\end{array}$

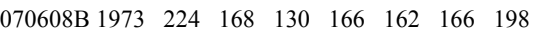

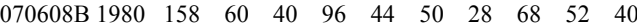

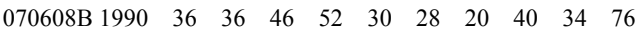

070608B $2000 \quad 42 \quad 46 \quad 34-9999$

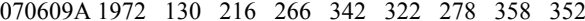

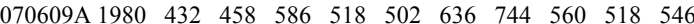

$\begin{array}{llllllllllll}070609 \text { A } 1990 & 634 & 546 & 572 & 654 & 510 & 540 & 632 & 468 & 314 & 324\end{array}$

070609A $2000 \quad 368 \quad 474 \quad 274-9999$

070609B $1977 \quad 68 \quad 292 \quad 324$

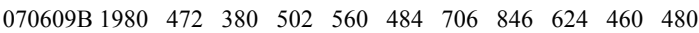

$\begin{array}{llllllllllll}070609 B & 1990 & 640 & 668 & 664 & 570 & 464 & 492 & 350 & 306 & 258 & 322\end{array}$

070609B 2000 $434 \quad 464 \quad 208-9999$

070610A 1979216

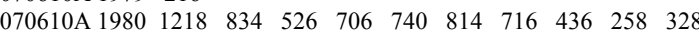

$\begin{array}{lllllllllll}070610 \text { A } 1990 & 288 & 236 & 182 & 210 & 256 & 238 & 202 & 212 & 154 & 116\end{array}$

070610A $2000 \quad 146 \quad 136 \quad 52-9999$

070610B $1977 \quad 704 \quad 472 \quad 692$

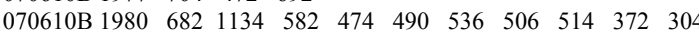

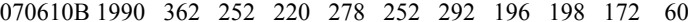

070610B 2000 $64 \quad 90 \quad 66-9999$

070611A $\begin{array}{lllll}1976 & 162 & 206 & 186 & 232\end{array}$

$\begin{array}{lllllllllll}070611 \text { A } 1980 & 222 & 346 & 252 & 336 & 342 & 316 & 312 & 246 & 274 & 220\end{array}$

$\begin{array}{llllllllllll}070611 \mathrm{~A} 1990 & 168 & 120 & 200 & 118 & 118 & 112 & 86 & 78 & 48 & 54\end{array}$

$\begin{array}{lllll}070611 \text { A } 2000 & 36 & 44 & 30-9999\end{array}$

070611B $1974 \quad 56 \quad 182 \quad 168 \quad 194 \quad 186 \quad 186$

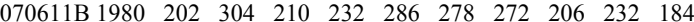

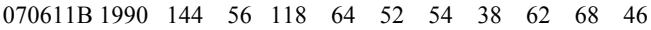

070611B $2000 \quad 36 \quad 34 \quad 30-9999$

070612A $1978 \quad 478 \quad 246$

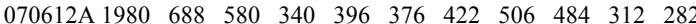

$\begin{array}{lllllllllll}070612 \text { A } 1990 & 196 & 180 & 138 & 82 & 120 & 136 & 80 & 106 & 44 & 20\end{array}$

070612A $2000 \quad 26 \quad 28 \quad 32-9999$

$070612 \mathrm{~B} 1979374$

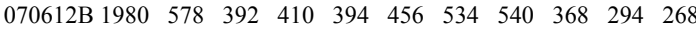

$\begin{array}{lllllllllll}070612 \mathrm{~B} 1990 & 208 & 242 & 238 & 202 & 192 & 138 & 92 & 88 & 46 & 76\end{array}$

070612B 2000 $44 \quad 48 \quad 64-9999$

070613A $1973 \quad 164 \quad 92 \quad 144 \quad 102 \quad 204 \quad 138 \quad 136$

$\begin{array}{lllllllllll}070613 \text { A } 1980 & 246 & 284 & 284 & 324 & 288 & 216 & 292 & 316 & 214 & 134\end{array}$

$\begin{array}{lllllllllll}070613 \text { A } 1990 & 234 & 256 & 310 & 280 & 292 & 272 & 176 & 124 & 74 & 66\end{array}$

070613A $2000 \quad 50 \quad 152 \quad 146-9999$

070613B $1973 \quad 166 \quad 142 \quad 134 \quad 166 \quad 262 \quad 214 \quad 270$

$\begin{array}{llllllllllll}070613 B & 1980 & 320 & 340 & 498 & 426 & 312 & 282 & 272 & 214 & 230 & 172\end{array}$

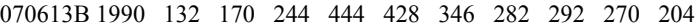
070613B 2000 $246 \quad 226 \quad 152-9999$

070614A $1975 \quad 194 \quad 244 \quad 240 \quad 310 \quad 252$

$\begin{array}{lllllllllll}070614 \text { A } 1980 & 292 & 286 & 240 & 262 & 234 & 254 & 280 & 202 & 172 & 188\end{array}$

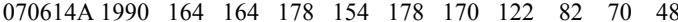

070614A $2000 \quad 48 \quad 40 \quad 36-9999$

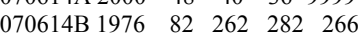

070614B 1980 $346 \begin{array}{lllllllll}296 & 350 & 380 & 352 & 368 & 374 & 298 & 204 & 290\end{array}$

$\begin{array}{lllllllllll}070614 \mathrm{~B} 1990 & 276 & 194 & 276 & 208 & 190 & 194 & 116 & 98 & 70 & 46\end{array}$

070614B $2000 \quad 38 \quad 34 \quad 34-9999$ 
070615A $1986 \quad 344 \quad 416 \quad 276 \quad 292$

$\begin{array}{lllllllllll}070615 \text { A } 1990 & 328 & 294 & 514 & 276 & 226 & 190 & 176 & 116 & 62 & 88\end{array}$ 070615A $2000 \quad 40 \quad 66 \quad 36-9999$

$\begin{array}{llllllllll}\text { 070616A } 1971 & 136 & 278 & 278 & 362 & 388 & 546 & 442 & 498 & 648\end{array}$ $\begin{array}{lllllllllll}070616 \text { A } 1980 & 468 & 578 & 346 & 322 & 196 & 118 & 80 & 140 & 86 & 86\end{array}$ $\begin{array}{lllllllllll}070616 \mathrm{~A} 1990 & 80 & 80 & 82 & 114 & 74 & 88 & 126 & 122 & 68 & 64\end{array}$ 070616A $2000 \quad 94 \quad 48 \quad 54-9999$

070617A $1984 \quad 156 \quad 158 \quad 228 \quad 260 \quad 164 \quad 132$

$\begin{array}{llllllllllll}070617 \mathrm{~A} & 1990 & 114 & 128 & 146 & 188 & 108 & 96 & 148 & 114 & 92 & 100\end{array}$ $\begin{array}{llllll}070617 \text { A } 2000 & 138 & 124 & 290 & -9999\end{array}$

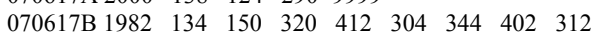

$\begin{array}{llllllllllll}070617 \mathrm{~B} & 1990 & 206 & 272 & 148 & 86 & 50 & 34 & 72 & 16 & 22 & 28\end{array}$

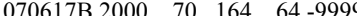

$\begin{array}{llllllll}070618 \text { A } 1973 & 558 & 612 & 384 & 728 & 766 & 802 & 894\end{array}$

$\begin{array}{lllllllllll}070618 \text { A } 1980 & 804 & 860 & 784 & 684 & 596 & 600 & 378 & 308 & 314 & 382\end{array}$

$\begin{array}{lllllllllll}070618 \text { A } 1990 & 308 & 366 & 222 & 204 & 142 & 130 & 88 & 104 & 64 & 58\end{array}$

070618A $2000 \quad 56 \quad 88 \quad 92-9999$

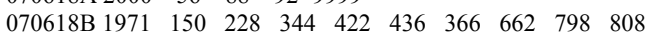

$\begin{array}{lllllllllll}070618 \mathrm{~B} 1980 & 884 & 694 & 620 & 592 & 590 & 516 & 550 & 294 & 166 & 342\end{array}$

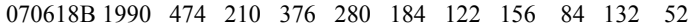

$\begin{array}{llllll}070618 B & 2000 & 58 & 64 & 38 & -9999\end{array}$

070619A $1972 \quad 102 \quad 132 \quad 272 \quad 318 \quad 260 \quad 282 \quad 190 \quad 130$

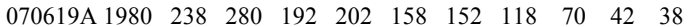

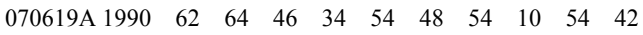

070619A $2000 \quad 28 \quad 56 \quad 46-9999$

070619B $1977 \quad 222 \quad 154 \quad 166$

$\begin{array}{lllllllllll}\text { 070619B } 1980 & 268 & 192 & 188 & 124 & 104 & 198 & 234 & 192 & 146 & 148\end{array}$

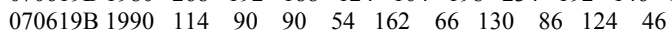

070619B $2000 \quad 54 \quad 130 \quad 58-9999$

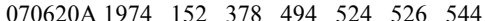

$\begin{array}{lllllllllll}070620 \mathrm{~A} 1980 & 440 & 434 & 562 & 488 & 396 & 524 & 476 & 360 & 186 & 322\end{array}$

$\begin{array}{llllllllllll}070620 \text { A } 1990 & 246 & 228 & 302 & 200 & 94 & 130 & 140 & 168 & 192 & 124\end{array}$

070620A $2000 \quad 82 \quad 248 \quad 110-9999$

070620B $1976 \quad 374 \quad 550 \quad 558 \quad 384$

$\begin{array}{lllllllllll}070620 \mathrm{~B} 1980 & 420 & 522 & 636 & 596 & 558 & 514 & 548 & 614 & 444 & 544\end{array}$

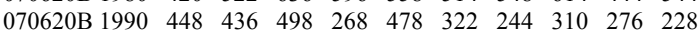

070620B $2000 \quad 262 \quad 180 \quad 56-9999$

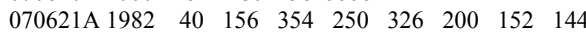

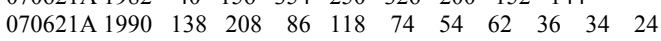

070621A $2000 \quad 20 \quad 32 \quad 42-9999$

070621B $1976 \quad 62 \quad 162 \quad 104 \quad 306$

$\begin{array}{lllllllllll}070621 \mathrm{~B} 1980 & 166 & 390 & 312 & 304 & 272 & 164 & 270 & 168 & 142 & 218\end{array}$

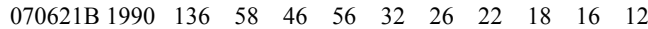

070621B $2000 \quad 28 \quad 20 \quad 26-9999$

070622A $1977 \quad 376 \quad 376 \quad 410$

$\begin{array}{lllllllllll}070622 \text { A } 1980 & 440 & 486 & 394 & 558 & 374 & 338 & 886 & 434 & 338 & 282\end{array}$

$\begin{array}{llllllllllll}070622 \mathrm{~A} 1990 & 228 & 308 & 210 & 244 & 348 & 456 & 292 & 298 & 242 & 494\end{array}$

070622A $2000 \quad 154 \quad 166 \quad 92-9999$

$\begin{array}{lllllll}070622 B & 1975 & 360 & 410 & 396 & 458 & 498\end{array}$

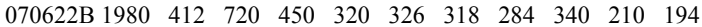

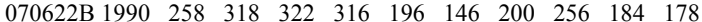

070622B $2000 \quad 98 \quad 168 \quad 76-9999$

070623A 1979368

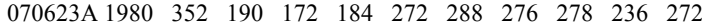

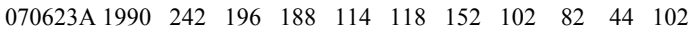

070623A $2000 \quad 40 \quad 60 \quad 140-9999$

070623B $1975 \quad 198 \quad 378 \quad 174 \quad 168 \quad 206$

$\begin{array}{llllllllllll}070623 B & 1980 & 260 & 226 & 336 & 230 & 212 & 246 & 210 & 178 & 90 & 64\end{array}$

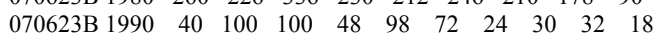

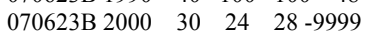

$\begin{array}{lllllll}070624 \mathrm{~A} & 1985 & 148 & 284 & 276 & 146 & 350\end{array}$

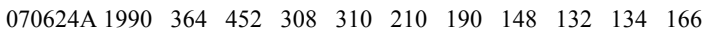

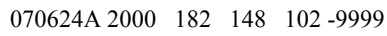

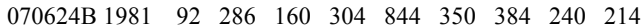

$\begin{array}{llllllllllll}070624 \mathrm{~B} 1990 & 164 & 246 & 262 & 196 & 158 & 154 & 98 & 122 & 104 & 98\end{array}$

070624B $2000 \quad 176 \quad 88 \quad 102-9999$

070625A $1974 \quad 66 \quad 46 \quad 58 \quad 20 \quad 28 \quad 56$

$\begin{array}{llllllllllll}070625 \mathrm{~A} & 1980 & 64 & 138 & 156 & 180 & 146 & 178 & 210 & 270 & 224 & 158\end{array}$

$\begin{array}{llllllllllll}070625 \mathrm{~A} & 1990 & 272 & 478 & 372 & 288 & 704 & 246 & 104 & 94 & 110 & 116\end{array}$

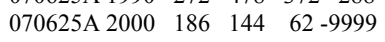

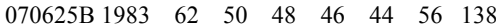

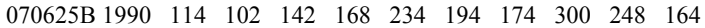

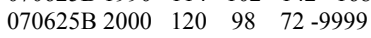

070626A $1973 \quad 202 \quad 374 \quad 168 \quad 210 \quad 316 \quad 460 \quad 406$

$\begin{array}{lllllllllll}070626 \text { A } 1980 & 592 & 434 & 488 & 538 & 464 & 514 & 496 & 350 & 130 & 144\end{array}$

$\begin{array}{lllllllllll}070626 \text { A } 1990 & 62 & 126 & 128 & 144 & 84 & 118 & 152 & 194 & 120 & 124\end{array}$

070626A $2000 \quad 86 \quad 132 \quad 112-9999$

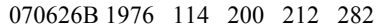

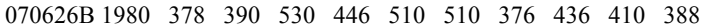

$\begin{array}{lllllllllll}070626 \mathrm{~B} 1990 & 280 & 338 & 288 & 228 & 172 & 168 & 152 & 134 & 174 & 116\end{array}$
070626B $2000 \quad 182 \quad 158 \quad 114-9999$

070627A $1979 \quad 152$

$\begin{array}{lllllllllll}070627 \mathrm{~A} 1980 & 224 & 168 & 290 & 304 & 562 & 334 & 378 & 346 & 310 & 350\end{array}$

$\begin{array}{llllllllllll}070627 \text { A } 1990 & 462 & 464 & 564 & 188 & 288 & 220 & 196 & 194 & 382 & 178\end{array}$ 070627A $2000 \quad 118 \quad 256 \quad 80-9999$

070627B $1978 \quad 210 \quad 330$

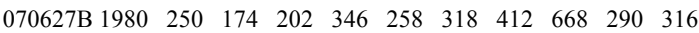

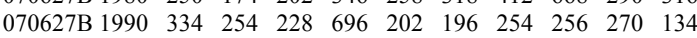

070627B $2000 \quad 204 \quad 278 \quad 144-9999$

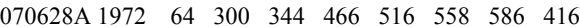

$\begin{array}{lllllllllll}070628 \text { A } 1980 & 520 & 320 & 254 & 206 & 180 & 236 & 182 & 216 & 88 & 82\end{array}$

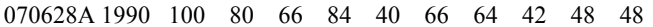

070628A $2000 \quad 46 \quad 50 \quad 30-9999$

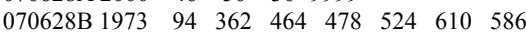

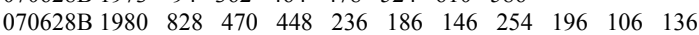

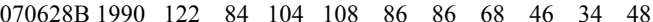

070628B $2000 \quad 48 \quad 52 \quad 50-9999$

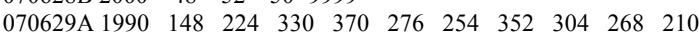

070629A $2000 \quad 160 \quad 166 \quad 202-9999$

070629B 1989252

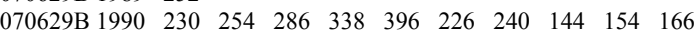
070629B $2000 \quad 134 \quad 94 \quad 68-9999$

070630A $1988 \quad 220 \quad 232$

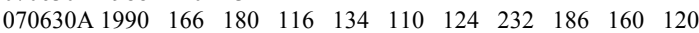

070630A $2000 \quad 180 \quad 114 \quad 220-9999$

070630B $1975 \quad 92 \quad 254 \quad 162 \quad 174 \quad 112$

070630B 1980 $186 \begin{array}{llllllllll}46 & 34 & 172 & 290 & 208 & 152 & 106 & 154 & 42\end{array}$

$\begin{array}{lllllllllll}070630 \mathrm{~B} 1990 & 22 & 20 & 18 & 54 & 14 & 10 & 30 & 32 & 10 & 10\end{array}$

070630B 2000 $10 \quad 14 \quad 14-9999$

$\begin{array}{llllllll}070631 \text { A } 1973 & 34 & 156 & 192 & 186 & 260 & 440 & 316\end{array}$

$\begin{array}{lllllllllll}070631 \mathrm{~A} 1980 & 204 & 236 & 212 & 634 & 584 & 312 & 332 & 190 & 186 & 284\end{array}$

$\begin{array}{lllllllllll}070631 \mathrm{~A} 1990 & 320 & 156 & 152 & 168 & 146 & 124 & 90 & 64 & 78 & 64\end{array}$

070631A $2000 \quad 56 \quad 66 \quad 32-9999$

070631B $1974 \quad 158 \quad 166 \quad 162 \quad 152 \quad 204 \quad 284$

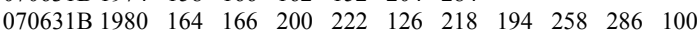

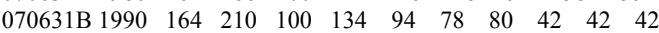

070631B $2000 \quad 38 \quad 52 \quad 76-9999$

070633A $1986 \quad 92 \quad 256 \quad 492 \quad 326$

$\begin{array}{lllllllllll}070633 \text { A } 1990 & 286 & 254 & 308 & 270 & 264 & 152 & 174 & 112 & 106 & 52\end{array}$

$\begin{array}{llllll}070633 \mathrm{~A} & 2000 & 64 & 60 & 60 & -9999\end{array}$

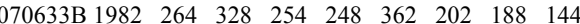

$\begin{array}{llllllllllll}070633 \mathrm{~B} & 1990 & 164 & 122 & 106 & 130 & 88 & 58 & 84 & 80 & 72 & 24\end{array}$

070633B $2000 \quad 38 \quad 32 \quad 50-9999$

070634A 1970 $34 \begin{array}{llllllllll}310 & 298 & 234 & 220 & 300 & 308 & 196 & 308 & 300\end{array}$

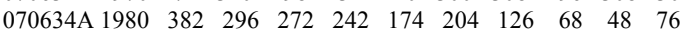

$\begin{array}{lllllllllll}070634 \mathrm{~A} 1990 & 80 & 62 & 92 & 84 & 96 & 88 & 98 & 88 & 124 & 100\end{array}$

070634A $2000 \quad 74 \quad 100 \quad 52-9999$

070634B $1975 \quad 230 \quad 278 \quad 266 \quad 456 \quad 304$

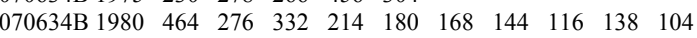

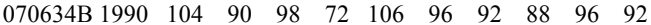

070634B $2000 \quad 118 \quad 96 \quad 50-9999$

070635A $1986 \quad 96 \quad 260 \quad 218 \quad 194$

$\begin{array}{lllllllllll}070635 \text { A } 1990 & 174 & 258 & 250 & 292 & 252 & 170 & 306 & 278 & 142 & 112\end{array}$

070635A $2000 \quad 116 \quad 112 \quad 100-9999$

070635B $1987 \quad 92 \quad 194 \quad 196$

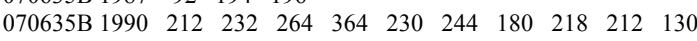

070635B $2000 \quad 114 \quad 62 \quad 262-9999$

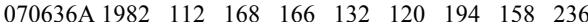

$\begin{array}{lllllllllll}070636 \text { A } 1990 & 126 & 144 & 116 & 88 & 88 & 140 & 82 & 112 & 40 & 22\end{array}$

$\begin{array}{lllll}070636 \text { A } 2000 & 22 & 22 & 52 & -9999\end{array}$

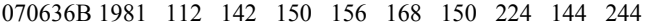

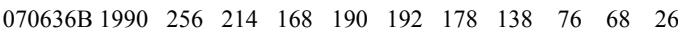

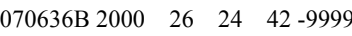

$\begin{array}{lllllllllll}070637 \mathrm{~A} 1980 & 210 & 500 & 232 & 204 & 204 & 196 & 232 & 230 & 206 & 126\end{array}$

$\begin{array}{llllllllllll}070637 \mathrm{~A} 1990 & 184 & 184 & 152 & 126 & 152 & 114 & 92 & 66 & 58 & 22\end{array}$

070637A $2000 \quad 36 \quad 26 \quad 42-9999$

070637B $1978 \quad 254 \quad 320$

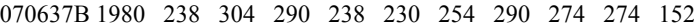

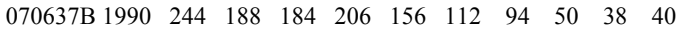
070637B $2000 \quad 28 \quad 42 \quad 34-9999$

070638A 1971 80 236 $196 \begin{array}{lllllll}170 & 192 & 182 & 270 & 264 & 302\end{array}$

$\begin{array}{lllllllllll}070638 \text { A } 1980 & 556 & 336 & 322 & 320 & 378 & 162 & 182 & 206 & 262 & 312\end{array}$

$\begin{array}{lllllllllll}070638 \text { A } 1990 & 310 & 202 & 288 & 188 & 208 & 232 & 234 & 196 & 168 & 102\end{array}$ 070638A $2000 \quad 120 \quad 156 \quad 84-9999$

$\begin{array}{llllllllll}070638 \text { B } 1971 & 184 & 292 & 244 & 204 & 222 & 222 & 198 & 308 & 294\end{array}$

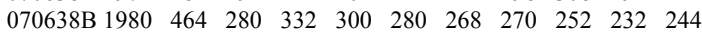

$\begin{array}{llllllllllll}070638 \mathrm{~B} 1990 & 308 & 194 & 198 & 172 & 158 & 146 & 146 & 126 & 104 & 92\end{array}$

070638B $2000 \quad 136 \quad 102 \quad 56-9999$

070639A 1979346

070639A 1980 $26 \begin{array}{llllllllll} & 156 & 202 & 200 & 198 & 132 & 146 & 118 & 298 & 432\end{array}$ 


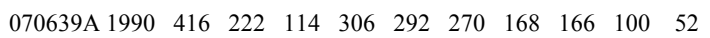
070639A $2000 \quad 74 \quad 62 \quad 66-9999$

070639B $1979 \quad 140$

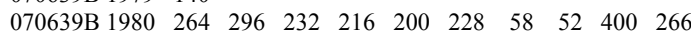

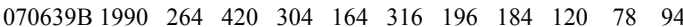
070639B 2000 $128 \quad 84 \quad 24-9999$

$\begin{array}{lllllllllll}070640 \text { A } 1980 & 216 & 156 & 124 & 168 & 128 & 108 & 102 & 132 & 200 & 92\end{array}$ $\begin{array}{lllllllllll}070640 \text { A } 1990 & 134 & 156 & 148 & 248 & 146 & 352 & 190 & 80 & 48 & 102\end{array}$ 070640A $2000 \quad 132 \quad 60 \quad 72-9999$

070640B 1979364

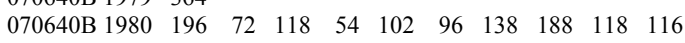

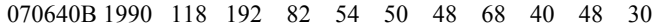

070640B $2000 \quad 78 \quad 56 \quad 50-9999$

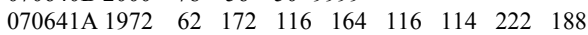

$\begin{array}{lllllllllll}070641 \text { A } 1980 & 244 & 238 & 278 & 318 & 258 & 294 & 262 & 300 & 170 & 178\end{array}$

$\begin{array}{llllllllllll}070641 \mathrm{~A} & 1990 & 178 & 182 & 220 & 152 & 220 & 194 & 128 & 142 & 162 & 104\end{array}$

070641A $2000 \quad 124 \quad 70 \quad 60-9999$

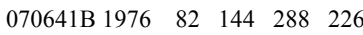

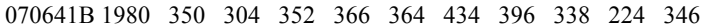

$\begin{array}{lllllllllll}070641 \mathrm{~B} 1990 & 366 & 326 & 366 & 302 & 356 & 328 & 308 & 312 & 348 & 180\end{array}$

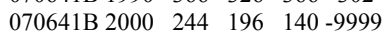

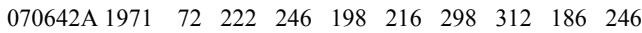

$\begin{array}{lllllllllll}070642 \text { A } 1980 & 192 & 186 & 218 & 166 & 120 & 152 & 84 & 120 & 136 & 114\end{array}$

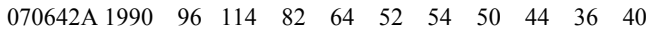

070642A $2000 \quad 44 \quad 40 \quad 34-9999$

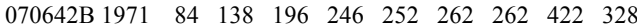

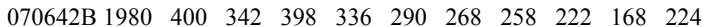

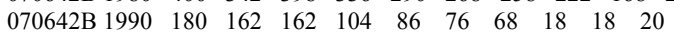

070642B $2000 \quad 34 \quad 26 \quad 32-9999$

$\begin{array}{lllllllllll}070643 \mathrm{~A} & 1971 & 92 & 328 & 236 & 246 & 230 & 192 & 204 & 290 & 270\end{array}$

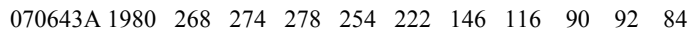

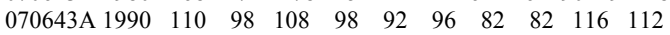

070643A $2000 \quad 70 \quad 78 \quad 44-9999$

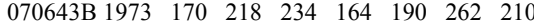

$\begin{array}{llllllllllll}070643 \mathrm{~B} & 1980 & 168 & 232 & 256 & 308 & 232 & 200 & 172 & 124 & 116 & 118\end{array}$

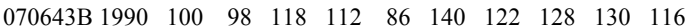

070643B $2000 \quad 98 \quad 78 \quad 64-9999$

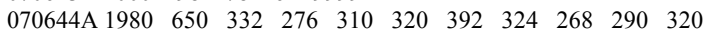

$\begin{array}{llllllllllll}070644 \mathrm{~A} & 1990 & 342 & 262 & 254 & 258 & 298 & 302 & 232 & 168 & 164 & 86\end{array}$

070644A $2000 \quad 126 \quad 152 \quad 60-9999$

070644B $1975 \quad 234 \quad 268 \quad 102 \quad 198 \quad 200$

$\begin{array}{lllllllllll}070644 \mathrm{~B} 1980 & 468 & 316 & 310 & 282 & 220 & 294 & 260 & 200 & 196 & 250\end{array}$

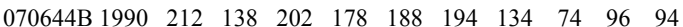

070644B $2000 \quad 154 \quad 100 \quad 64-9999$

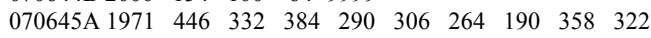

$\begin{array}{llllllllllll}070645 \text { A } 1980 & 622 & 390 & 258 & 314 & 274 & 320 & 364 & 296 & 230 & 272\end{array}$

$\begin{array}{llllllllllll}070645 \text { A } 1990 & 358 & 282 & 310 & 278 & 258 & 232 & 148 & 134 & 194 & 134\end{array}$

070645A $2000 \quad 156 \quad 178 \quad 100-9999$

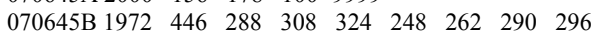

$\begin{array}{llllllllllll}070645 B & 1980 & 438 & 204 & 254 & 244 & 228 & 194 & 220 & 224 & 172 & 174\end{array}$

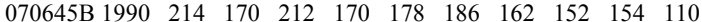

070645B $2000 \quad 104 \quad 144 \quad 60-9999$

$\begin{array}{lllllllll}070646 \text { A } 1972 & 200 & 328 & 268 & 200 & 184 & 222 & 230 & 192\end{array}$

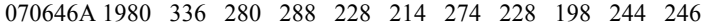

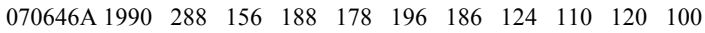

070646A $2000 \quad 118 \quad 108 \quad 72-9999$

070646B $1976 \quad 170 \quad 252 \quad 202 \quad 148$

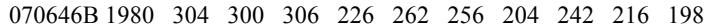

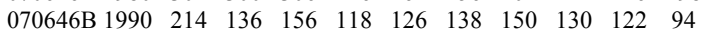

070646B $2000 \quad 76 \quad 86 \quad 70-9999$

070647A $1979 \quad 86$

$\begin{array}{lllllllllll}\text { 070647A } 1980 & 214 & 190 & 204 & 216 & 240 & 298 & 194 & 398 & 254 & 202\end{array}$

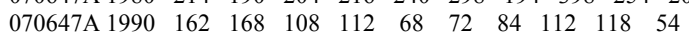

070647A $2000 \quad 42 \quad 34 \quad 38-9999$

070647B 1979486

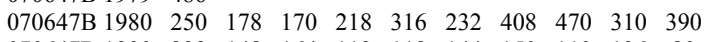

$\begin{array}{lllllllllll}070647 \mathrm{~B} 1990 & 222 & 148 & 164 & 112 & 118 & 144 & 150 & 110 & 136 & 80\end{array}$

070647B $2000 \quad 74 \quad 98 \quad 36-9999$

070648A $1984 \quad 96 \quad 254 \quad 194 \quad 188 \quad 150 \quad 152$

$\begin{array}{lllllllllll}070648 \text { A } 1990 & 232 & 210 & 242 & 126 & 132 & 138 & 80 & 98 & 72 & 50\end{array}$

070648A $2000 \quad 46 \quad 34 \quad 32-9999$

070649A $1989 \quad 90$

$\begin{array}{lllllllllll}070649 \text { A } 1990 & 224 & 238 & 160 & 242 & 252 & 210 & 260 & 178 & 124 & 368\end{array}$

070649A $2000 \quad 350 \quad 126 \quad 114-9999$

070649B $1987 \quad 318 \quad 256 \quad 288$

$\begin{array}{lllllllllll}070649 \mathrm{~B} 1990 & 274 & 288 & 268 & 128 & 154 & 184 & 442 & 432 & 270 & 278\end{array}$

070649B 2000 $398 \quad 316 \quad 276-9999$

$\begin{array}{llllll}070650 \mathrm{~A} & 1986 & 58 & 126 & 320 & 376\end{array}$

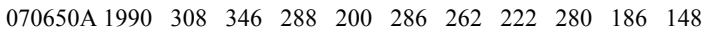

070650A $2000 \quad 160 \quad 118 \quad 62-9999$
070650B $1988 \quad 72 \quad 352$

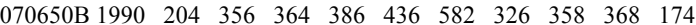
070650B $2000 \quad 138 \quad 64 \quad 80-9999$

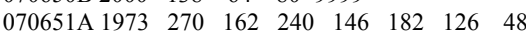

070651A 1980 $104 \begin{array}{llllllllll}136 & 162 & 120 & 60 & 132 & 132 & 50 & 46 & 60\end{array}$

$\begin{array}{lllllllllll}070651 \mathrm{~A} 1990 & 68 & 58 & 94 & 100 & 66 & 72 & 64 & 66 & 58 & 48\end{array}$

$\begin{array}{lllll}070651 \text { A } 2000 & 102 & 44 & 82 & -9999\end{array}$

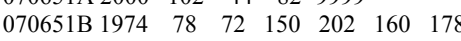

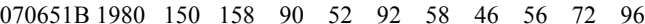

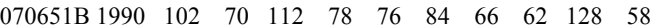

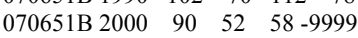

$\begin{array}{lllllllll}070653 \text { A } 1972 & 118 & 196 & 240 & 214 & 168 & 204 & 330 & 202\end{array}$

$\begin{array}{lllllllllll}070653 \text { A } 1980 & 280 & 320 & 302 & 220 & 262 & 282 & 318 & 256 & 116 & 148\end{array}$

$\begin{array}{llllllllllll}070653 \text { A } 1990 & 160 & 140 & 166 & 162 & 136 & 124 & 74 & 64 & 60 & 38\end{array}$

$\begin{array}{lllll}070653 \text { A } 2000 & 38 & 48 & 36 & -9999\end{array}$

070653B $1976 \quad 312 \quad 402 \quad 244 \quad 454$

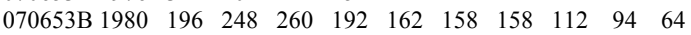

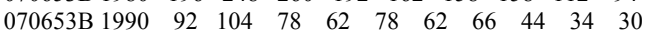

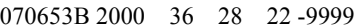

070654A $1972 \quad 148 \quad 154 \quad 246 \quad 156 \quad 224 \quad 178 \quad 276 \quad 280$

$\begin{array}{lllllllllllll}070654 \mathrm{~A} 1980 & 258 & 226 & 234 & 272 & 222 & 258 & 158 & 98 & 108 & 60\end{array}$

$\begin{array}{llllllllllll}070654 \mathrm{~A} & 1990 & 70 & 50 & 114 & 54 & 134 & 108 & 138 & 50 & 76 & 98\end{array}$

070654A $2000 \quad 98 \quad 96 \quad 132-9999$

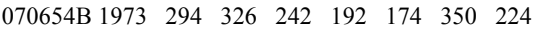

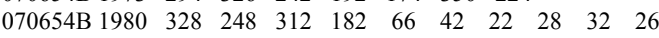

$\begin{array}{lllllllllll}070654 \mathrm{~B} 1990 & 54 & 34 & 82 & 110 & 96 & 56 & 68 & 82 & 112 & 90\end{array}$

070654B 2000 $112 \quad 122 \quad 68-9999$

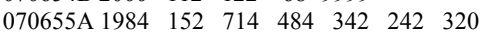

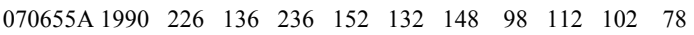

070655A $2000 \quad 82 \quad 56 \quad 68-9999$

$\begin{array}{llllllllll}070655 \mathrm{~B} & 1982 & 156 & 498 & 564 & 612 & 516 & 452 & 244 & 284\end{array}$

$\begin{array}{lllllllllll}070655 \mathrm{~B} 1990 & 230 & 104 & 170 & 178 & 180 & 202 & 146 & 152 & 86 & 90\end{array}$

070655B $2000 \quad 82 \quad 72 \quad 34-9999$

070656A 1979138

$\begin{array}{lllllllllll}070656 \text { A } 1980 & 130 & 124 & 152 & 132 & 128 & 168 & 148 & 172 & 184 & 330\end{array}$

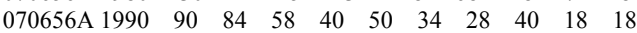

070656A $2000 \quad 22 \quad 20 \quad 20-9999$

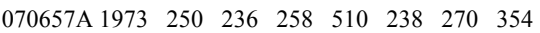

$\begin{array}{llllllllllll}070657 \mathrm{~A} & 1980 & 368 & 364 & 248 & 258 & 378 & 506 & 378 & 490 & 322 & 154\end{array}$

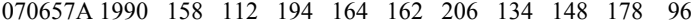

070657A $2000 \quad 94 \quad 72 \quad 58-9999$

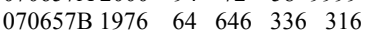

$\begin{array}{lllllllllll}070657 \mathrm{~B} 1980 & 274 & 278 & 276 & 232 & 216 & 272 & 324 & 268 & 314 & 256\end{array}$

$\begin{array}{llllllllllll}070657 \mathrm{~B} & 1990 & 196 & 116 & 162 & 104 & 94 & 110 & 268 & 166 & 78 & 120\end{array}$

070657B $2000 \quad 166 \quad 104 \quad 204-9999$

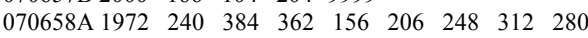

$\begin{array}{lllllllllll}070658 \text { A } 1980 & 342 & 314 & 326 & 350 & 330 & 328 & 356 & 206 & 108 & 254\end{array}$

$\begin{array}{llllllllllll}070658 \text { A } 1990 & 310 & 198 & 320 & 196 & 220 & 202 & 164 & 238 & 302 & 196\end{array}$

$\begin{array}{lllll}070658 \text { A } 2000 & 258 & 216 & 166 & -9999\end{array}$

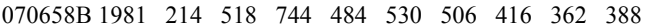

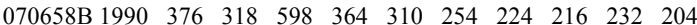

070658B $2000 \quad 124 \quad 152 \quad 188-9999$

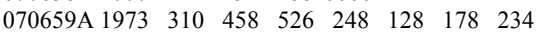

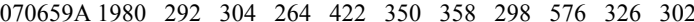

$\begin{array}{lllllllllll}070659 \text { A } 1990 & 332 & 186 & 128 & 120 & 176 & 190 & 168 & 100 & 92 & 124\end{array}$

070659A $2000 \quad 198 \quad 14 \quad 68-9999$

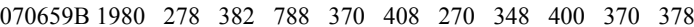

070659B 1990 $308 \begin{array}{llllllllll}300 & 254 & 306 & 204 & 328 & 216 & 206 & 184 & 168\end{array}$

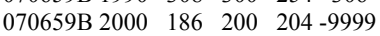

070660A $1975 \quad 670 \quad 418 \quad 478 \quad 536 \quad 426$

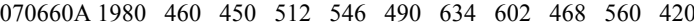

070660A $1990 \quad 472 \quad 644 \quad 728 \quad 546 \quad 506 \quad 484 \quad 276 \quad 200 \quad 206 \quad 214$

$\begin{array}{lllll}070660 \text { A } 2000 & 216 & 238 & 152 & -9999\end{array}$

070660B $1976 \quad 728 \quad 462 \quad 496 \quad 550$

070660B 1980 $47 \begin{array}{llllllllll}528 & 542 & 612 & 472 & 510 & 632 & 624 & 594 & 442\end{array}$

070660B 1990 $476 \begin{array}{llllllllll}400 & 410 & 310 & 270 & 224 & 230 & 156 & 230 & 242\end{array}$

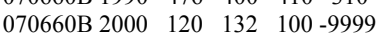

070661A $1975 \quad 448 \quad 476 \quad 526 \quad 742 \quad 722$

$\begin{array}{lllllllllll}070661 \text { A } 1980 & 610 & 628 & 758 & 736 & 656 & 710 & 550 & 208 & 180 & 252\end{array}$

$\begin{array}{lllllllllll}070661 \text { A } 1990 & 354 & 210 & 224 & 162 & 174 & 174 & 122 & 116 & 146 & 74\end{array}$

070661A $2000 \quad 114 \quad 176 \quad 84-9999$

070661B 1971 $140 \begin{array}{lllllllll}202 & 220 & 530 & 612 & 554 & 676 & 826 & 620\end{array}$

$\begin{array}{llllllllllll}070661 \mathrm{~B} 1980 & 646 & 566 & 660 & 656 & 642 & 588 & 552 & 258 & 178 & 252\end{array}$

$\begin{array}{lllllllllll}070661 \mathrm{~B} 1990 & 320 & 212 & 276 & 210 & 248 & 220 & 194 & 162 & 182 & 90\end{array}$

070661B $2000 \quad 108 \quad 138 \quad 46-9999$

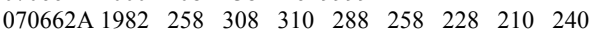

$\begin{array}{lllllllllll}070662 \text { A } 1990 & 192 & 466 & 172 & 102 & 86 & 44 & 24 & 122 & 48 & 34\end{array}$

070662A $2000 \quad 52 \quad 36 \quad 38-9999$

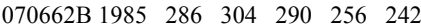

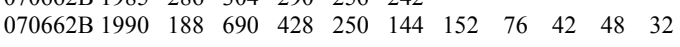


070662B $2000 \quad 82 \quad 92 \quad 88-9999$

$\begin{array}{llllllllll}070663 \text { A } 1981 & 488 & 250 & 158 & 112 & 40 & 108 & 100 & 102 & 58\end{array}$

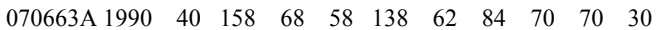
$\begin{array}{lllll}070663 \text { A } 2000 & 34 & 68 & 50 & -9999\end{array}$

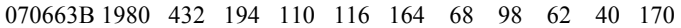

070663B $1990 \quad 40 \quad 48 \quad 52 \quad 72 \quad 50 \quad 42 \quad 52 \quad 52 \quad 48 \quad 54$

070663B $2000 \quad 84 \quad 48 \quad 76-9999$

070664A $1974 \quad 84 \quad 498 \quad 658 \quad 874 \quad 790 \quad 856$

$\begin{array}{lllllllllll}070664 \text { A } 1980 & 816 & 674 & 568 & 544 & 504 & 610 & 416 & 284 & 70 & 644\end{array}$

$\begin{array}{lllllllllll}070664 \text { A } 1990 & 92 & 178 & 202 & 184 & 194 & 112 & 168 & 230 & 140 & 206\end{array}$

$\begin{array}{lllll}070664 \text { A } 2000 & 64 & 196 & 106 & -9999\end{array}$

070664B $1974 \quad 160 \quad 374 \quad 410 \quad 416 \quad 490 \quad 652$

070664B 1980 $64 \begin{array}{llllllllll}546 & 568 & 420 & 402 & 630 & 550 & 620 & 506 & 430\end{array}$

$\begin{array}{lllllllllll}070664 \mathrm{~B} 1990 & 292 & 296 & 258 & 418 & 272 & 200 & 178 & 142 & 112 & 176\end{array}$

070664B $2000 \quad 220 \quad 284 \quad 338-9999$

\section{Watershed 13}

130101A $1954 \quad 54 \quad 56 \quad 84 \quad 80 \quad 24 \quad 32$

$\begin{array}{lllllllllll}130101 \mathrm{~A} 1960 & 30 & 44 & 130 & 218 & 206 & 236 & 170 & 232 & 258 & 326\end{array}$

$\begin{array}{lllllllllll}130101 \mathrm{~A} 1970 & 388 & 168 & 144 & 144 & 152 & 282 & 226 & 228 & 190 & 196\end{array}$

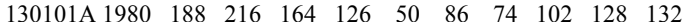

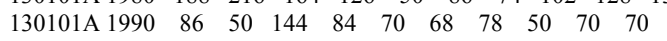

130101A $2000 \quad 62 \quad 66 \quad 40-9999$

130101B $1949 \quad 24$

130101B 1950 $46 \begin{array}{llllllllll}38 & 86 & 84 & 124 & 66 & 56 & 52 & 72 & 66\end{array}$

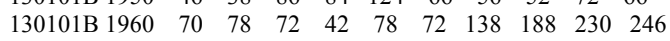

$\begin{array}{lllllllllll}130101 \mathrm{~B} 1970 & 270 & 208 & 216 & 162 & 136 & 376 & 258 & 190 & 142 & 148\end{array}$

$\begin{array}{lllllllllll}130101 \mathrm{~B} 1980 & 164 & 184 & 112 & 176 & 164 & 124 & 52 & 110 & 212 & 110\end{array}$

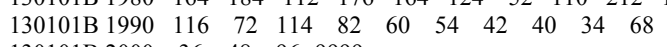

130101B $2000 \quad 36 \quad 48 \quad 96-9999$

130102A $1909 \quad 136$

$\begin{array}{lllllllllll}130102 \mathrm{~A} 1910 & 178 & 298 & 224 & 196 & 264 & 234 & 292 & 258 & 276 & 368\end{array}$ $\begin{array}{llllllllllll}130102 \mathrm{~A} 1920 & 236 & 314 & 288 & 392 & 430 & 286 & 264 & 184 & 164 & 142\end{array}$

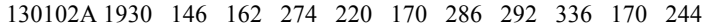
$\begin{array}{llllllllllll}130102 \mathrm{~A} 1940 & 386 & 214 & 230 & 248 & 198 & 134 & 86 & 76 & 136 & 148\end{array}$

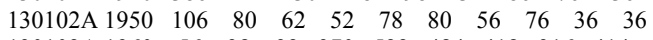

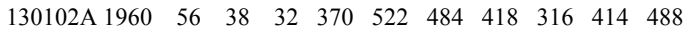
$\begin{array}{lllllllllll}130102 \mathrm{~A} 1970 & 380 & 372 & 508 & 400 & 400 & 432 & 332 & 374 & 356 & 354\end{array}$ $\begin{array}{lllllllllll}130102 \mathrm{~A} 1980 & 370 & 328 & 506 & 430 & 410 & 428 & 368 & 432 & 260 & 196\end{array}$

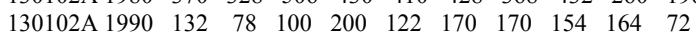
130102A $2000 \quad 38 \quad 72 \quad 46-9999$

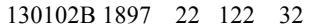

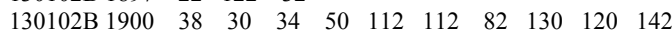

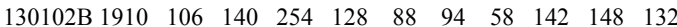

130102B 1920 $14 \begin{array}{llllllllll}158 & 196 & 230 & 226 & 236 & 256 & 290 & 336 & 282\end{array}$

$\begin{array}{lllllllllll}130102 \mathrm{~B} 1930 & 160 & 176 & 170 & 98 & 146 & 236 & 258 & 136 & 176 & 142\end{array}$

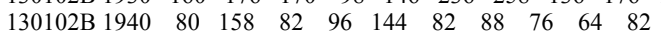

130102B 1950 $6 \begin{array}{lllllllllll}60 & 70 & 50 & 102 & 64 & 62 & 42 & 56 & 46 & 68\end{array}$

130102B $1960 \begin{array}{lllllllllll}290 & 586 & 558 & 430 & 410 & 298 & 322 & 378 & 236 & 404\end{array}$

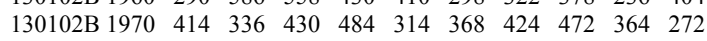

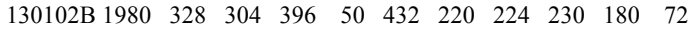

$\begin{array}{lllllllllll}130102 \mathrm{~B} 1990 & 44 & 92 & 74 & 114 & 80 & 102 & 86 & 108 & 92 & 66\end{array}$

130102B 2000 $24 \quad 44 \quad 44-9999$

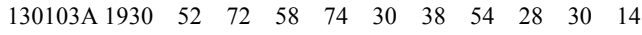

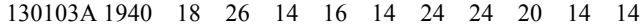

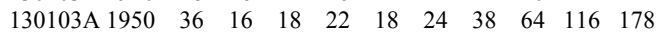

$\begin{array}{lllllllllll}130103 \text { A } 1960 & 256 & 344 & 302 & 252 & 262 & 242 & 204 & 204 & 160 & 248\end{array}$

$\begin{array}{lllllllllll}130103 \mathrm{~A} 1970 & 132 & 132 & 100 & 122 & 106 & 168 & 182 & 156 & 252 & 140\end{array}$

$\begin{array}{lllllllllll}130103 \text { A } 1980 & 228 & 290 & 188 & 88 & 116 & 74 & 64 & 56 & 46 & 86\end{array}$

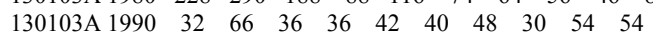

130103A $2000 \quad 136 \quad 186 \quad 92-9999$

130103B $1938 \quad 30 \quad 40$

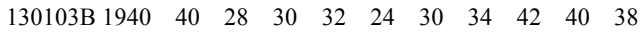

$\begin{array}{lllllllllll}130103 \mathrm{~B} 1950 & 18 & 14 & 12 & 12 & 24 & 38 & 36 & 34 & 34 & 32\end{array}$

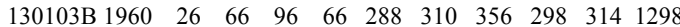

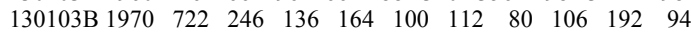

$\begin{array}{lllllllllll}130103 \mathrm{~B} 1980 & 284 & 218 & 132 & 110 & 96 & 60 & 60 & 60 & 40 & 88\end{array}$

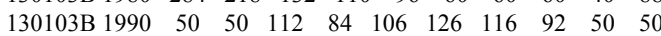

130103B 2000 $174 \quad 116 \quad 66-9999$

130104A 1952 $38 \quad 70 \quad 40 \quad 56 \quad 76 \quad 74 \quad 72 \quad 42$

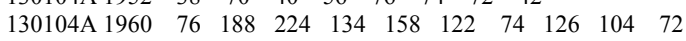

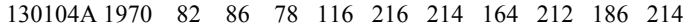

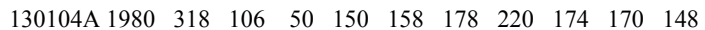

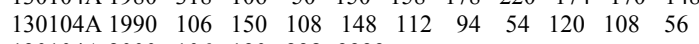

130104A $2000 \quad 106 \quad 190 \quad 238-9999$

$\begin{array}{llllllllll}\text { 130104B } 1961 & 322 & 180 & 180 & 122 & 118 & 82 & 114 & 188 & 122\end{array}$
130104B 1970 $148 \begin{array}{llllllllll}214 & 150 & 136 & 94 & 234 & 190 & 228 & 160 & 320\end{array}$

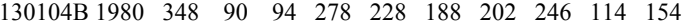
$\begin{array}{lllllllllll}130104 \mathrm{~B} 1990 & 116 & 204 & 234 & 152 & 68 & 98 & 92 & 128 & 122 & 134\end{array}$ 130104B 2000 $120 \quad 144 \quad 56-9999$

$\begin{array}{llllllllll}130105 \mathrm{~A} 1951 & 38 & 72 & 50 & 84 & 64 & 122 & 108 & 252 & 372\end{array}$

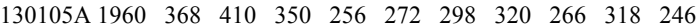

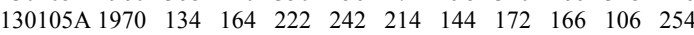
$\begin{array}{llllllllllll}130105 \text { A } 1980 & 262 & 216 & 220 & 130 & 158 & 148 & 106 & 224 & 144 & 188\end{array}$

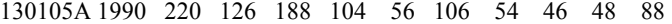
130105A 2000 $112 \quad 212 \quad 134-9999$

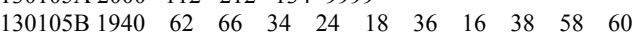

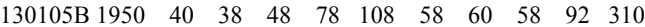

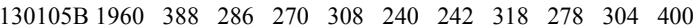
$\begin{array}{llllllllllll}130105 \mathrm{~B} 1970 & 304 & 194 & 198 & 268 & 280 & 218 & 130 & 192 & 114 & 96\end{array}$

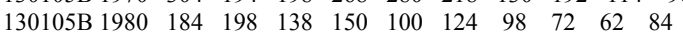

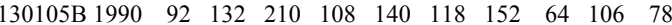
130105B $2000 \quad 138 \quad 178 \quad 88-9999$

130106A $1968 \quad 286 \quad 324$

130106A 1970 $396 \begin{array}{lllllllll}414 & 356 & 272 & 146 & 98 & 330 & 418 & 248 & 240\end{array}$ $\begin{array}{lllllllllll}\text { 130106A } 1980 & 158 & 174 & 190 & 156 & 88 & 62 & 44 & 52 & 228 & 142\end{array}$ $\begin{array}{llllllllllll}130106 \mathrm{~A} & 1990 & 196 & 102 & 144 & 98 & 70 & 128 & 100 & 56 & 92 & 36\end{array}$ 130106A $2000 \quad 42 \quad 112 \quad 70-9999$

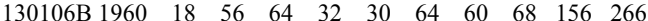
$\begin{array}{lllllllllll}\text { 130106B } 1970 & 184 & 198 & 242 & 126 & 114 & 70 & 180 & 146 & 138 & 130\end{array}$

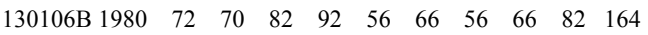

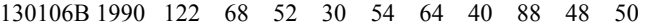
130106B $2000 \quad 30 \quad 40 \quad 38-9999$

130108A $1944 \quad 110 \quad 132 \quad 128 \quad 188 \quad 64 \quad 88$

$\begin{array}{lllllllllll}130108 \mathrm{~A} 1950 & 44 & 44 & 54 & 84 & 34 & 56 & 62 & 60 & 40 & 38\end{array}$

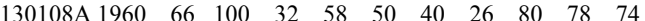

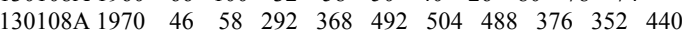

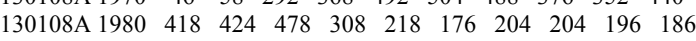

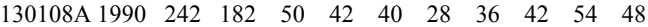
130108A $2000 \quad 80 \quad 132 \quad 128-9999$

130108B $1958 \quad 62 \quad 124$

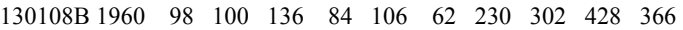

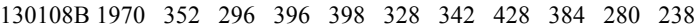
$\begin{array}{lllllllllll}130108 \mathrm{~B} 1980 & 228 & 218 & 132 & 174 & 130 & 52 & 46 & 56 & 26 & 48\end{array}$

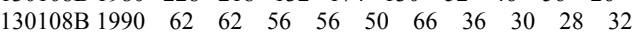
130108B 2000 $14 \quad 14 \quad 16-9999$

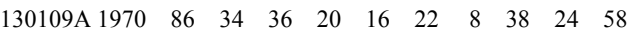
130109A 1980 $36 \begin{array}{lllllllllll}36 & 298 & 302 & 250 & 288 & 330 & 170 & 242 & 194 & 180\end{array}$

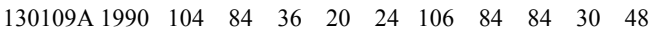
130109A $2000 \quad 40 \quad 68 \quad 68-9999$

130109B 1979218

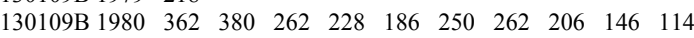

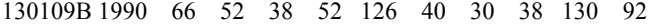
130109B 2000 $152 \quad 144 \quad 52-9999$

130110A $1945 \quad 84 \quad 40 \quad 38 \quad 78 \quad 52$

130110A 1950 $44 \begin{array}{llllllllll}92 & 28 & 44 & 106 & 34 & 38 & 36 & 46 & 44\end{array}$

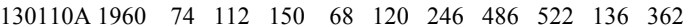
130110A $1970 \begin{array}{lllllllllll}236 & 206 & 370 & 190 & 272 & 224 & 248 & 380 & 442 & 508\end{array}$ $\begin{array}{llllllllllll}130110 \text { A } 1980 & 468 & 416 & 404 & 344 & 440 & 366 & 396 & 362 & 304 & 62\end{array}$

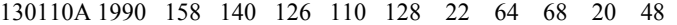
130110A $2000 \quad 82 \quad 60 \quad 76-9999$

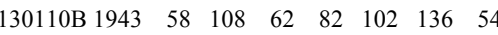

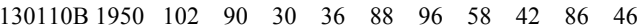

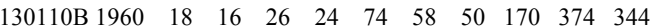

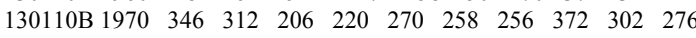
$\begin{array}{lllllllllllll}130110 \mathrm{~B} & 1980 & 294 & 348 & 312 & 340 & 408 & 386 & 302 & 100 & 88 & 46\end{array}$

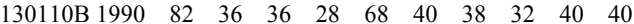
130110B $2000 \quad 46 \quad 40 \quad 32-9999$

130111A $1956 \quad 134 \quad 234 \quad 134 \quad 204$

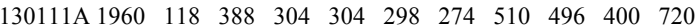

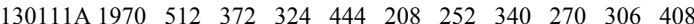
$\begin{array}{lllllllllll}130111 \mathrm{~A} 1980 & 374 & 330 & 316 & 288 & 266 & 298 & 226 & 136 & 438 & 514\end{array}$ $\begin{array}{lllllllllll}130111 \mathrm{~A} 1990 & 250 & 298 & 188 & 342 & 286 & 218 & 186 & 170 & 98 & 12\end{array}$ 130111A $2000 \quad 338 \quad 262 \quad 158-9999$

130111B $1966 \quad 430 \quad 226 \quad 262 \quad 170$

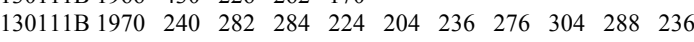

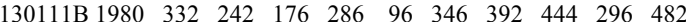

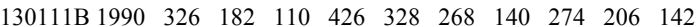
130111B 2000 $168 \begin{array}{lll}150 & 78 & -9999\end{array}$

$\begin{array}{llllllll}130112 \text { A } 1973 & 130 & 112 & 338 & 250 & 252 & 184 & 138\end{array}$

$\begin{array}{lllllllllll}130112 \mathrm{~A} 1980 & 202 & 146 & 192 & 192 & 132 & 86 & 44 & 60 & 34 & 38\end{array}$

$\begin{array}{lllllllllll}130112 \mathrm{~A} 1990 & 56 & 30 & 122 & 128 & 32 & 14 & 24 & 32 & 10 & 14\end{array}$

130112A $2000 \quad 16 \quad 28 \quad 48-9999$

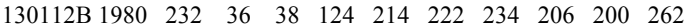

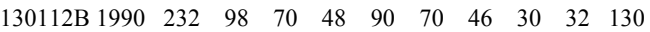

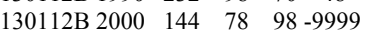


130113A $1955 \quad 40 \quad 38 \quad 258 \quad 534 \quad 430$

$\begin{array}{lllllllllll}130113 \mathrm{~A} 1960 & 504 & 442 & 256 & 354 & 386 & 286 & 348 & 208 & 248 & 290\end{array}$

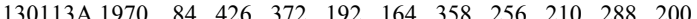

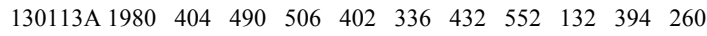
$\begin{array}{llllllllllll}130113 \mathrm{~A} 1990 & 208 & 320 & 270 & 278 & 118 & 216 & 206 & 102 & 74 & 74\end{array}$

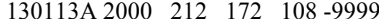

130113B $1957 \quad 164 \quad 480 \quad 614$

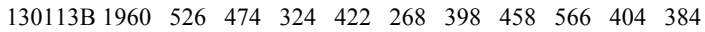
130113B 1970 $27 \begin{array}{llllllllll}288 & 224 & 152 & 188 & 250 & 110 & 320 & 176 & 342\end{array}$

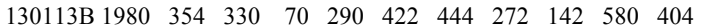

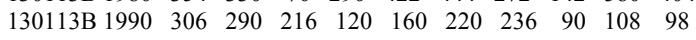
130113B $2000 \quad 184 \quad 166 \quad 60-9999$

$\begin{array}{lllllll}130114 \mathrm{~A} & 1915 & 270 & 392 & 562 & 316 & 104\end{array}$

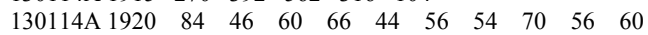
$\begin{array}{lllllllllll}130114 \mathrm{~A} 1930 & 36 & 102 & 82 & 92 & 46 & 78 & 86 & 94 & 66 & 62\end{array}$

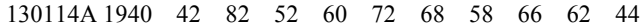
$\begin{array}{lllllllllll}130114 \mathrm{~A} 1950 & 42 & 32 & 20 & 26 & 12 & 24 & 8 & 22 & 30 & 26\end{array}$ $\begin{array}{lllllllllll}130114 \mathrm{~A} 1960 & 36 & 40 & 50 & 268 & 230 & 268 & 244 & 196 & 20 & 112\end{array}$

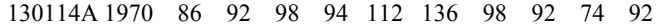
$\begin{array}{lllllllllll}130114 \mathrm{~A} 1980 & 94 & 66 & 58 & 54 & 94 & 82 & 44 & 50 & 54 & 30\end{array}$ $\begin{array}{llllllllllll}130114 \mathrm{~A} 1990 & 18 & 28 & 40 & 24 & 28 & 20 & 26 & 28 & 14 & 26\end{array}$ 130114A $2000 \quad 26 \quad 14 \quad 24-9999$

130114B $1909 \quad 184$

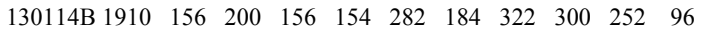
$\begin{array}{lllllllllll}130114 \mathrm{~B} 1920 & 102 & 92 & 80 & 94 & 180 & 192 & 168 & 160 & 120 & 172\end{array}$

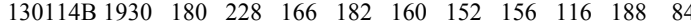

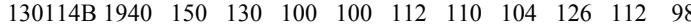
$\begin{array}{lllllllllll}130114 \mathrm{~B} 1950 & 76 & 82 & 58 & 76 & 68 & 82 & 76 & 84 & 64 & 62\end{array}$ 130114B 1960 $36 \begin{array}{llllllllll}76 & 236 & 350 & 392 & 330 & 348 & 72 & 50 & 218 & 310\end{array}$

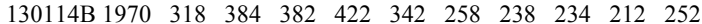
$\begin{array}{lllllllllll}130114 \mathrm{~B} 1980 & 158 & 194 & 184 & 240 & 196 & 188 & 176 & 200 & 120 & 66\end{array}$ $\begin{array}{lllllllllll}130114 \mathrm{~B} 1990 & 70 & 180 & 168 & 132 & 136 & 98 & 114 & 82 & 118 & 106\end{array}$ 130114B 2000 $46 \quad 118 \quad 82-9999$

130201A $1907 \quad 192 \quad 266 \quad 170$

$\begin{array}{lllllllllll}130201 \mathrm{~A} 1910 & 148 & 112 & 106 & 172 & 108 & 166 & 130 & 150 & 122 & 114\end{array}$

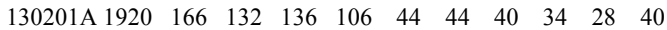
$\begin{array}{llllllllllll}130201 \mathrm{~A} & 1930 & 90 & 90 & 168 & 210 & 270 & 234 & 192 & 196 & 190 & 204\end{array}$

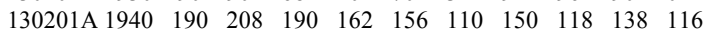

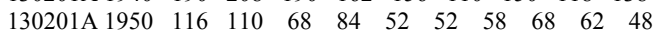
$\begin{array}{llllllllllll}130201 \mathrm{~A} 1960 & 44 & 90 & 90 & 102 & 102 & 98 & 70 & 100 & 108 & 82\end{array}$

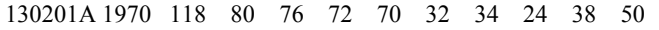
$\begin{array}{llllllllllll}130201 \mathrm{~A} & 1980 & 100 & 122 & 158 & 192 & 138 & 176 & 164 & 138 & 86 & 88\end{array}$

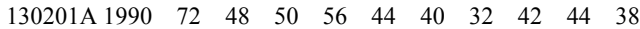

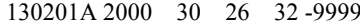

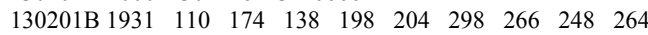
$\begin{array}{llllllllllll}130201 \mathrm{~B} 1940 & 220 & 214 & 168 & 158 & 134 & 138 & 146 & 126 & 138 & 144\end{array}$ 130201B 1950 $170 \begin{array}{llllllllll}116 & 80 & 116 & 78 & 80 & 52 & 70 & 60 & 76\end{array}$ $\begin{array}{lllllllllll}130201 \mathrm{~B} 1960 & 70 & 102 & 120 & 106 & 136 & 140 & 98 & 130 & 192 & 182\end{array}$

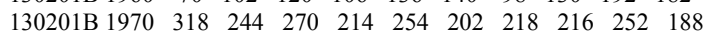

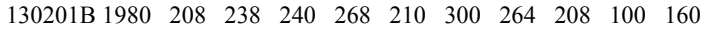

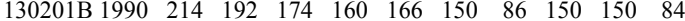
130201B $2000 \quad 156 \quad 154 \quad 114-9999$

130202 A $1928 \quad 150 \quad 230$

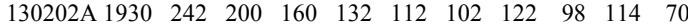

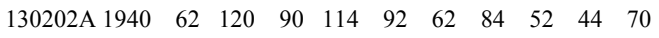
$\begin{array}{lllllllllll}130202 \mathrm{~A} 1950 & 72 & 78 & 68 & 106 & 60 & 82 & 80 & 112 & 142 & 176\end{array}$

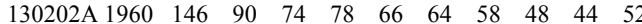

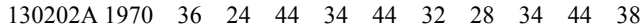
$\begin{array}{lllllllllll}130202 \mathrm{~A} 1980 & 32 & 26 & 34 & 28 & 34 & 32 & 40 & 42 & 36 & 30\end{array}$ $\begin{array}{lllllllllll}130202 \mathrm{~A} 1990 & 36 & 44 & 32 & 36 & 48 & 54 & 36 & 40 & 24 & 44\end{array}$ 130202A $2000 \quad 30 \quad 28 \quad 30-9999$

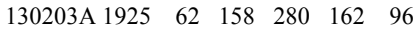

$\begin{array}{lllllllllll}130203 \mathrm{~A} 1930 & 66 & 60 & 70 & 108 & 82 & 120 & 128 & 100 & 72 & 126\end{array}$

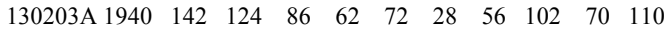

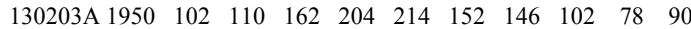
$\begin{array}{lllllllllll}130203 \text { A } 1960 & 92 & 86 & 60 & 52 & 80 & 66 & 70 & 62 & 42 & 22\end{array}$ $\begin{array}{lllllllllll}130203 \mathrm{~A} 1970 & 30 & 24 & 30 & 14 & 24 & 30 & 18 & 20 & 36 & 36\end{array}$ $\begin{array}{lllllllllll}130203 \mathrm{~A} 1980 & 54 & 68 & 52 & 78 & 80 & 54 & 58 & 44 & 30 & 14\end{array}$

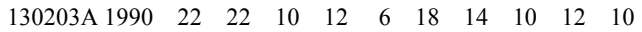
$\begin{array}{lllll}130203 \text { A } 2000 & 8 & 8 & 12 & -9999\end{array}$

130203B 1930 $44 \begin{array}{llllllllll}44 & 64 & 58 & 58 & 84 & 180 & 186 & 204 & 130 & 146\end{array}$

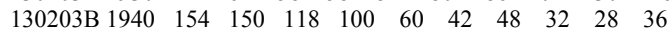
$\begin{array}{lllllllllll}130203 \mathrm{~B} 1950 & 100 & 98 & 172 & 168 & 182 & 156 & 158 & 134 & 138 & 134\end{array}$

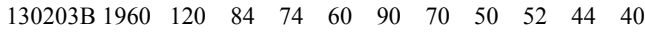
130203B 1970 $40 \begin{array}{llllllllll}40 & 24 & 24 & 28 & 16 & 18 & 20 & 24 & 30 & 44\end{array}$ $\begin{array}{lllllllllll}130203 \mathrm{~B} 1980 & 54 & 32 & 46 & 54 & 54 & 52 & 52 & 56 & 36 & 52\end{array}$

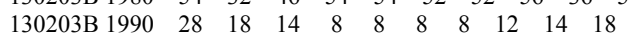
130203B 2000 $12 \quad 16 \quad 14-9999$

130204A $1945 \quad 60 \quad 156 \quad 198 \quad 178 \quad 220$

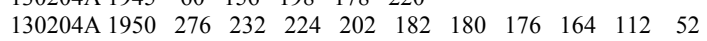

130204A $1960 \quad 48 \quad 46 \quad 48 \quad 82 \quad 82 \quad 96 \quad 100 \quad 92 \quad 126 \quad 102$

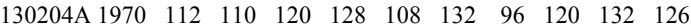

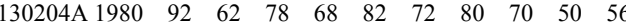
$\begin{array}{lllllllllll}130204 \mathrm{~A} 1990 & 56 & 46 & 54 & 38 & 44 & 42 & 34 & 36 & 34 & 38\end{array}$ 130204A $2000 \quad 26 \quad 36 \quad 38-9999$

$130204 \mathrm{~B} 1948 \quad 58 \quad 156$

130204B 1950 $142 \begin{array}{llllllllll}156 & 150 & 142 & 122 & 80 & 56 & 50 & 46 & 42\end{array}$ $\begin{array}{lllllllllll}130204 \mathrm{~B} 1960 & 64 & 98 & 98 & 136 & 126 & 188 & 154 & 154 & 196 & 266\end{array}$

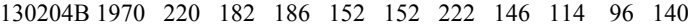
130204B 1980 $92 \begin{array}{llllllllll} & 102 & 100 & 94 & 78 & 76 & 58 & 64 & 60 & 44\end{array}$ 130204B 1990 $446 \begin{array}{llllllllll}58 & 48 & 56 & 34 & 50 & 54 & 66 & 58 & 70\end{array}$ 130204B 2000 $52 \quad 68 \quad 54-9999$

$130205 \mathrm{~B} 1945 \quad 318 \quad 416 \quad 194 \quad 224 \quad 242$

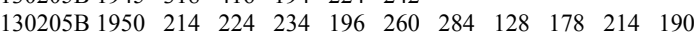
130205B $1960 \begin{array}{lllllllllll}240 & 260 & 228 & 152 & 170 & 230 & 254 & 250 & 224 & 238\end{array}$ $\begin{array}{lllllllllll}130205 \mathrm{~B} 1970 & 290 & 330 & 354 & 256 & 238 & 224 & 330 & 262 & 278 & 190\end{array}$

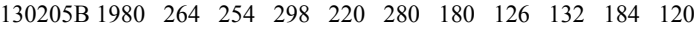

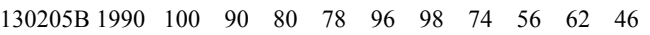
130205B 2000 $62 \quad 50 \quad 88-9999$

130206A $1966 \quad 140 \quad 184 \quad 138 \quad 146$

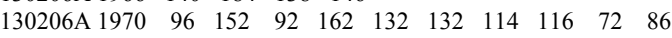

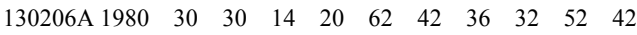

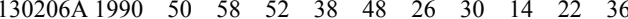
130206A $2000 \quad 32 \quad 26 \quad 18-9999$

130206B $1957 \quad 74 \quad 138 \quad 122$

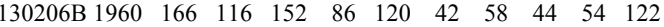

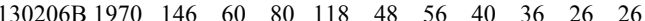

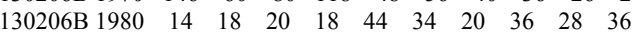

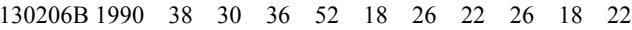
130206B $2000 \quad 72 \quad 36 \quad 26-9999$

$\begin{array}{lllllllllll}130207 \mathrm{~A} 1930 & 46 & 90 & 96 & 98 & 84 & 104 & 76 & 82 & 108 & 78\end{array}$ $\begin{array}{lllllllllll}130207 \mathrm{~A} 1940 & 72 & 66 & 60 & 46 & 44 & 34 & 44 & 198 & 236 & 162\end{array}$

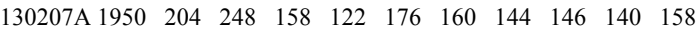
130207A $1960 \begin{array}{lllllllllll}120 & 104 & 92 & 92 & 64 & 56 & 42 & 32 & 26 & 20\end{array}$ $\begin{array}{lllllllllll}130207 \mathrm{~A} 1970 & 14 & 18 & 24 & 12 & 14 & 26 & 22 & 18 & 24 & 34\end{array}$ $\begin{array}{lllllllllll}130207 \mathrm{~A} 1980 & 40 & 94 & 86 & 70 & 110 & 86 & 122 & 148 & 118 & 102\end{array}$

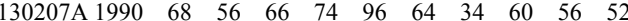
130207A $2000 \quad 34 \quad 42 \quad 42-9999$ 130208A 192948

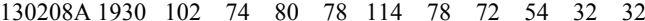

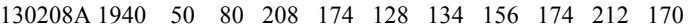

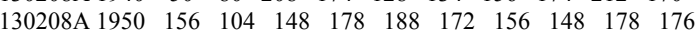
$\begin{array}{lllllllllll}130208 \text { A } 1960 & 148 & 128 & 142 & 114 & 166 & 156 & 114 & 100 & 102 & 74\end{array}$

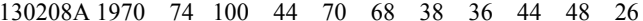
$\begin{array}{lllllllllll}130208 \mathrm{~A} 1980 & 28 & 26 & 38 & 54 & 52 & 64 & 38 & 82 & 50 & 36\end{array}$

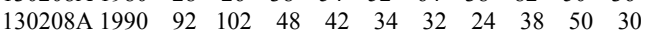
130208A $2000 \quad 30 \quad 26 \quad 52-9999$

130208B $1928 \quad 38 \quad 216$

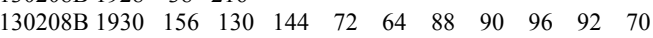
130208B 1940 $34 \begin{array}{llllllllll}50 & 36 & 26 & 28 & 30 & 60 & 158 & 138 & 124\end{array}$

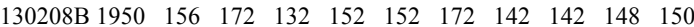

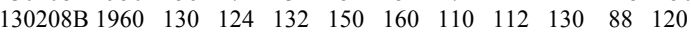

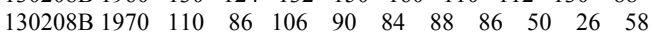

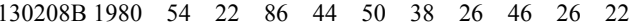

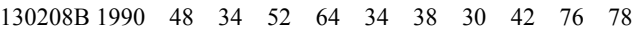
130208B $2000 \quad 66 \quad 54 \quad 62-9999$

130209A $1937 \quad 202 \quad 158 \quad 132$

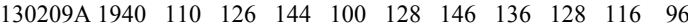
$\begin{array}{lllllllllll}130209 \text { A } 1950 & 74 & 84 & 90 & 84 & 118 & 150 & 154 & 156 & 160 & 166\end{array}$

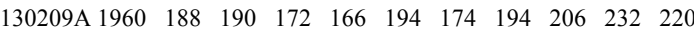
130209A 1970 $248 \begin{array}{llllllllll}296 & 210 & 200 & 302 & 258 & 214 & 212 & 274 & 182\end{array}$ $\begin{array}{lllllllllll}130209 \text { A } 1980 & 248 & 174 & 204 & 228 & 200 & 210 & 278 & 226 & 158 & 162\end{array}$

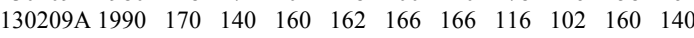
130209A 2000 $164 \quad 180 \quad 96-9999$

130209B $1935 \quad 178 \quad 286 \quad 316 \quad 266 \quad 202$

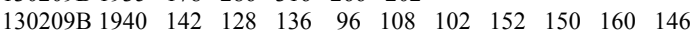
130209B 1950 $166 \begin{array}{llllllllll}148 & 118 & 144 & 60 & 90 & 62 & 82 & 60 & 68\end{array}$

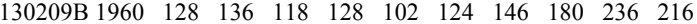

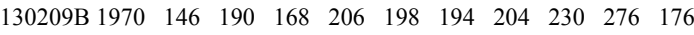

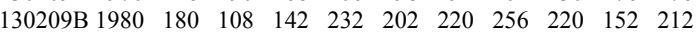

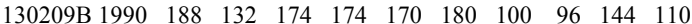
130209B 2000 $120 \quad 102 \quad 66-9999$

130210A $1946 \quad 238 \quad 190 \quad 104 \quad 66$ $\begin{array}{lllllllllll}130210 \mathrm{~A} 1950 & 40 & 44 & 24 & 28 & 26 & 24 & 12 & 16 & 22 & 16\end{array}$ $\begin{array}{lllllllllll}130210 \mathrm{~A} 1960 & 22 & 18 & 20 & 42 & 56 & 84 & 134 & 96 & 190 & 168\end{array}$ $\begin{array}{llllllllllll}130210 \mathrm{~A} & 1970 & 106 & 124 & 150 & 106 & 78 & 98 & 92 & 74 & 42 & 14\end{array}$ $\begin{array}{lllllllllll}130210 \text { A } 1980 & 26 & 28 & 24 & 22 & 26 & 54 & 136 & 134 & 98 & 92\end{array}$

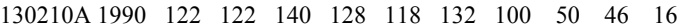
130210A $2000 \quad 16 \quad 16 \quad 14-9999$

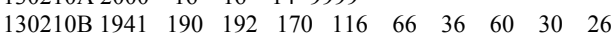




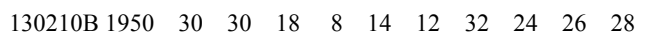

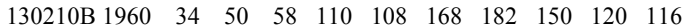
$\begin{array}{lllllllllll}130210 \mathrm{~B} 1970 & 104 & 96 & 116 & 126 & 102 & 40 & 8 & 10 & 12 & 14\end{array}$

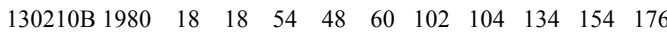
$\begin{array}{lllllllllll}130210 \mathrm{~B} 1990 & 148 & 128 & 80 & 102 & 38 & 28 & 18 & 14 & 8 & 14\end{array}$

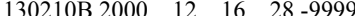

130211A $1938 \quad 102 \quad 334$

$\begin{array}{lllllllllll}130211 \mathrm{~A} 1940 & 566 & 382 & 298 & 326 & 258 & 208 & 324 & 356 & 218 & 172\end{array}$

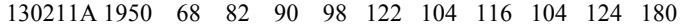

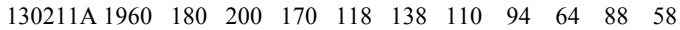

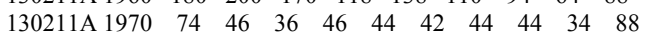

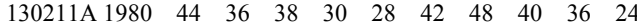

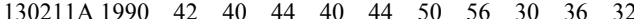
130211A $2000 \quad 36 \quad 26 \quad 44-9999$

130211B $1926 \quad 578 \quad 322 \quad 280 \quad 224$

$\begin{array}{lllllllllll}130211 \mathrm{~B} 1930 & 290 & 340 & 246 & 170 & 154 & 98 & 70 & 112 & 118 & 122\end{array}$

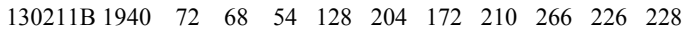

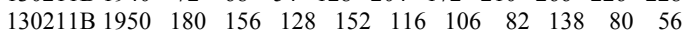
$\begin{array}{llllllllllll}130211 \mathrm{~B} & 1960 & 88 & 108 & 114 & 120 & 128 & 60 & 54 & 116 & 110 & 68\end{array}$ $\begin{array}{lllllllllll}130211 \mathrm{~B} 1970 & 90 & 68 & 142 & 128 & 72 & 64 & 70 & 72 & 88 & 96\end{array}$

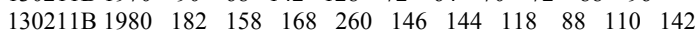

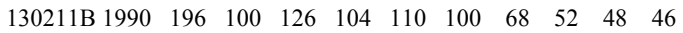
130211B 2000 $20 \quad 42 \quad 64-9999$

130214A $1963 \quad 190 \quad 118 \quad 108 \quad 94 \quad 98 \quad 112 \quad 100$

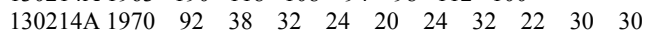
$\begin{array}{lllllllllll}130214 \mathrm{~A} 1980 & 44 & 74 & 144 & 208 & 162 & 70 & 112 & 100 & 86 & 68\end{array}$ $\begin{array}{lllllllllll}130214 \mathrm{~A} 1990 & 44 & 32 & 32 & 16 & 26 & 26 & 22 & 26 & 16 & 20\end{array}$ 130214A $2000 \quad 34 \quad 20 \quad 30-9999$

130214B $1966 \quad 94 \quad 136 \quad 186 \quad 112$

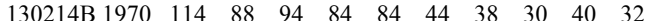
130214B 1980 $20 \begin{array}{llllllllll}16 & 48 & 54 & 40 & 22 & 42 & 80 & 120 & 198\end{array}$

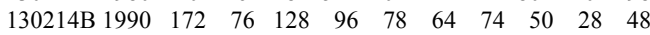
130214B $2000 \quad 78 \quad 46 \quad 50-9999$

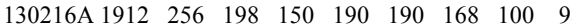
$\begin{array}{llllllllllll}130216 \text { A } 1920 & 148 & 136 & 174 & 108 & 100 & 84 & 58 & 64 & 74 & 90\end{array}$

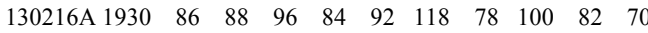

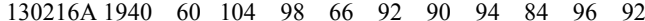

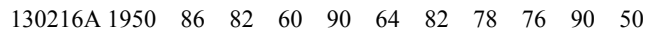
$\begin{array}{lllllllllll}130216 \mathrm{~A} 1960 & 42 & 50 & 82 & 140 & 216 & 232 & 296 & 290 & 160 & 50\end{array}$

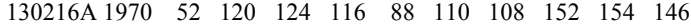
$\begin{array}{lllllllllll}130216 \text { A } 1980 & 182 & 168 & 170 & 178 & 174 & 156 & 152 & 190 & 136 & 162\end{array}$

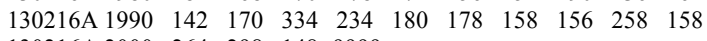
130216A $2000 \quad 264 \quad 298 \quad 148-9999$

130216B $1938 \quad 94 \quad 72$

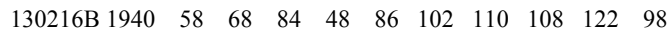

$\begin{array}{lllllllllll}130216 \mathrm{~B} 1950 & 82 & 70 & 50 & 58 & 56 & 64 & 62 & 52 & 64 & 46\end{array}$

130216B 1960 $38 \quad 40 \quad 50 \quad 122 \quad 236 \quad 192 \quad 262 \quad 276 \quad 192 \quad 66$

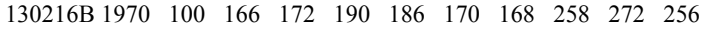

$\begin{array}{lllllllllll}130216 \mathrm{~B} 1980 & 328 & 322 & 374 & 332 & 342 & 312 & 294 & 288 & 168 & 264\end{array}$

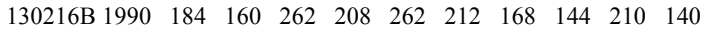

130216B $2000 \quad 196 \quad 196 \quad 118-9999$

130217A $1954 \quad 160 \quad 194 \quad 102 \quad 74 \quad 54 \quad 40$

$\begin{array}{lllllllllll}130217 \mathrm{~A} 1960 & 22 & 34 & 22 & 18 & 34 & 104 & 66 & 64 & 68 & 98\end{array}$

130217A 1970 $18 \begin{array}{llllllllll}78 & 28 & 36 & 34 & 34 & 62 & 78 & 70 & 144 & 266\end{array}$

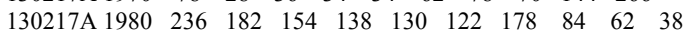

$\begin{array}{lllllllllll}130217 \mathrm{~A} 1990 & 22 & 52 & 32 & 22 & 18 & 18 & 18 & 12 & 12 & 18\end{array}$

130217A $2000 \quad 18 \quad 18 \quad 22-9999$

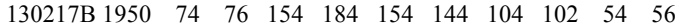

$\begin{array}{lllllllllll}130217 \mathrm{~B} 1960 & 44 & 52 & 38 & 24 & 34 & 44 & 34 & 16 & 22 & 28\end{array}$

$\begin{array}{lllllllllll}130217 \mathrm{~B} 1970 & 64 & 82 & 78 & 66 & 62 & 48 & 36 & 68 & 112 & 148\end{array}$

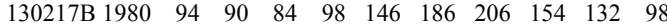

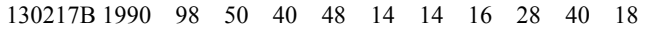

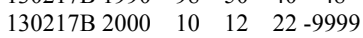

130218A $1937 \quad 106 \quad 168 \quad 124$

$\begin{array}{lllllllllll}130218 \text { A } 1940 & 96 & 46 & 24 & 18 & 16 & 16 & 22 & 14 & 16 & 8\end{array}$

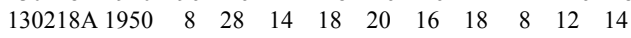

$\begin{array}{lllllllllllll}130218 \text { A } 1960 & 18 & 6 & 6 & 4 & 6 & 8 & 8 & 42 & 172 & 218\end{array}$

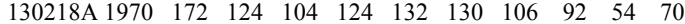

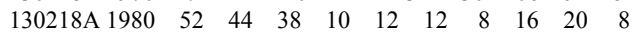

$\begin{array}{lllllllllll}130218 \mathrm{~A} 1990 & 6 & 20 & 12 & 14 & 8 & 10 & 12 & 10 & 8 & 8\end{array}$

130218A $2000 \quad 10 \quad 10 \quad 14-9999$

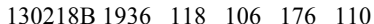

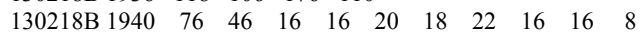

$\begin{array}{lllllllllll}130218 \mathrm{~B} 1950 & 4 & 10 & 12 & 24 & 16 & 14 & 26 & 8 & 10 & 10\end{array}$

$\begin{array}{lllllllllll}130218 \mathrm{~B} 1960 & 22 & 16 & 16 & 16 & 14 & 14 & 12 & 14 & 72 & 122\end{array}$

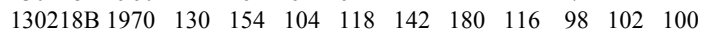

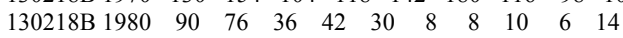

$\begin{array}{lllllllllll}130218 \mathrm{~B} 1990 & 14 & 6 & 6 & 4 & 8 & 4 & 10 & 12 & 10 & 10\end{array}$

130218B 2000 $10 \quad 14 \quad 24-9999$

130219A $1943 \quad 46 \quad 78 \quad 62 \quad 56 \quad 66 \quad 76 \quad 30$

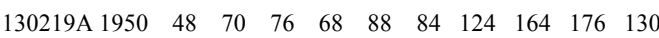

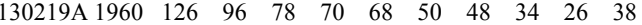

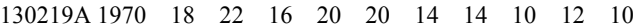

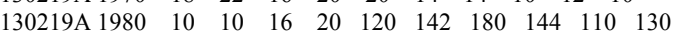

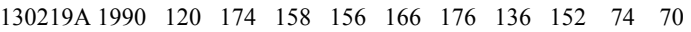
130219A $2000 \quad 70 \quad 94 \quad 70-9999$

130219B 1949120

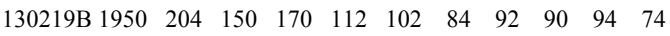
130219B 1960 $64 \begin{array}{llllllllll}46 & 20 & 26 & 26 & 20 & 26 & 26 & 26 & 16\end{array}$ 130219B 1970 $24 \quad \begin{array}{llllllllll}68 & 28 & 36 & 148 & 168 & 256 & 178 & 114 & 192\end{array}$

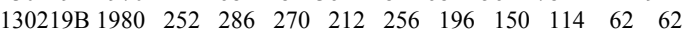
$\begin{array}{lllllllllll}\text { 130219B } 1990 & 56 & 74 & 12 & 12 & 10 & 14 & 16 & 12 & 10 & 18\end{array}$ 130219B $2000 \quad 12 \quad 10 \quad 38-9999$

130220A $1926260 \quad 202 \quad 242 \quad 250$

$\begin{array}{lllllllllll}130220 \text { A } 1930 & 354 & 278 & 294 & 208 & 168 & 118 & 184 & 178 & 240 & 148\end{array}$

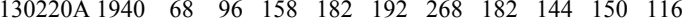

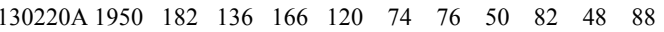

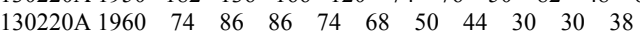

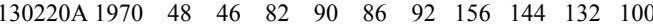
$\begin{array}{lllllllllll}130220 \text { A } 1980 & 112 & 124 & 132 & 156 & 90 & 132 & 116 & 126 & 64 & 36\end{array}$ $\begin{array}{lllllllllll}130220 \mathrm{~A} 1990 & 40 & 56 & 32 & 38 & 52 & 52 & 50 & 26 & 18 & 24\end{array}$ 130220A $2000 \quad 24 \quad 20 \quad 38-9999$

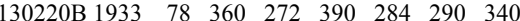

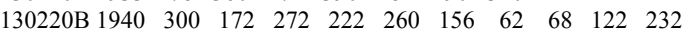
$\begin{array}{lllllllllll}130220 \mathrm{~B} 1950 & 244 & 418 & 212 & 148 & 110 & 88 & 96 & 86 & 134 & 92\end{array}$ 130220B 1960 $64 \begin{array}{llllllllll}64 & 68 & 58 & 54 & 32 & 34 & 44 & 50 & 46 & 32\end{array}$ 130220B 1970 $44 \begin{array}{llllllllll}38 & 58 & 30 & 32 & 26 & 28 & 28 & 34 & 66\end{array}$

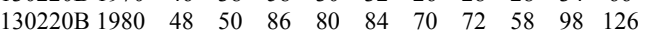

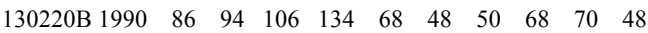
130220B $2000 \quad 70 \quad 86 \quad 58-9999$

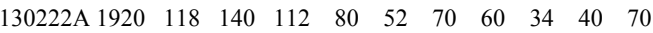
$\begin{array}{lllllllllll}130222 \mathrm{~A} 1930 & 46 & 18 & 16 & 26 & 30 & 34 & 40 & 52 & 50 & 38\end{array}$

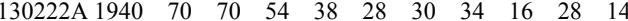
$\begin{array}{lllllllllll}130222 \mathrm{~A} 1950 & 36 & 52 & 26 & 16 & 28 & 16 & 38 & 38 & 24 & 16\end{array}$ $\begin{array}{lllllllllll}130222 \mathrm{~A} 1960 & 40 & 62 & 100 & 104 & 74 & 80 & 66 & 58 & 94 & 86\end{array}$

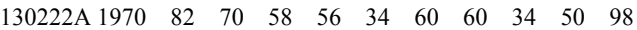
$\begin{array}{lllllllllll}130222 \mathrm{~A} 1980 & 62 & 98 & 72 & 58 & 26 & 52 & 74 & 36 & 42 & 46\end{array}$

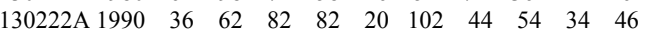
130222A $2000 \quad 46 \quad 32 \quad 44-9999$

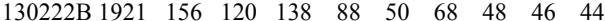

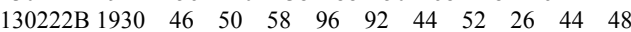

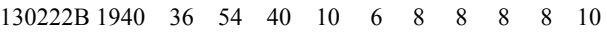

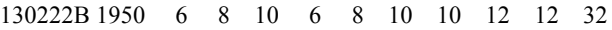

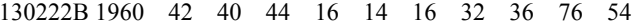
$\begin{array}{lllllllllll}130222 \mathrm{~B} 1970 & 56 & 38 & 38 & 62 & 78 & 80 & 72 & 52 & 72 & 60\end{array}$ $\begin{array}{lllllllllll}130222 \mathrm{~B} 1980 & 70 & 64 & 76 & 42 & 32 & 90 & 62 & 70 & 64 & 50\end{array}$ $\begin{array}{lllllllllll}130222 \mathrm{~B} 1990 & 40 & 32 & 38 & 30 & 36 & 38 & 28 & 46 & 50 & 52\end{array}$ $130222 \mathrm{~B} 2000 \quad 36 \quad 40 \quad 44-9999$

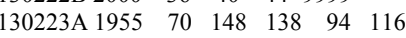

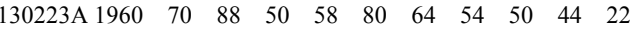

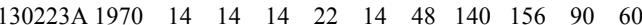
$\begin{array}{lllllllllll}130223 \text { A } 1980 & 20 & 24 & 18 & 20 & 20 & 18 & 24 & 22 & 16 & 16\end{array}$ $\begin{array}{lllllllllll}130223 \text { A } 1990 & 22 & 24 & 22 & 22 & 32 & 50 & 58 & 36 & 18 & 22\end{array}$ 130223A $2000 \quad 18 \quad 24 \quad 26-9999$

130223B $1978 \quad 198 \quad 166$

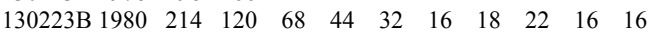
$\begin{array}{lllllllllll}130223 \mathrm{~B} 1990 & 24 & 18 & 36 & 30 & 56 & 76 & 52 & 40 & 50 & 28\end{array}$ $130223 \mathrm{~B} 2000 \quad 36 \quad 32 \quad 26-9999$

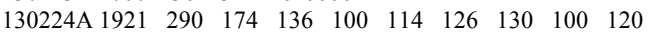
$\begin{array}{lllllllllll}130224 \mathrm{~A} 1930 & 164 & 138 & 92 & 146 & 128 & 188 & 168 & 194 & 154 & 136\end{array}$

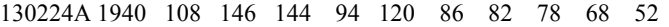

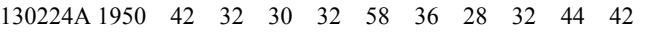

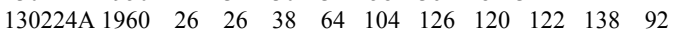

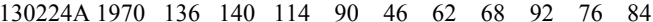
$\begin{array}{lllllllllll}130224 \mathrm{~A} 1980 & 84 & 94 & 112 & 128 & 78 & 58 & 48 & 80 & 54 & 36\end{array}$ $\begin{array}{lllllllllll}130224 \mathrm{~A} 1990 & 42 & 34 & 22 & 28 & 32 & 30 & 28 & 16 & 28 & 16\end{array}$ $\begin{array}{lllll}130224 \mathrm{~A} & 2000 & 18 & 14 & 16-9999\end{array}$

130224B $1924 \quad 192 \quad 162 \quad 110 \quad 146 \quad 102 \quad 88$

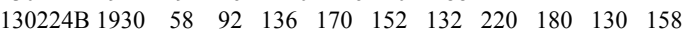

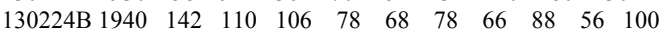
130224B 1950 $64 \begin{array}{llllllllll}60 & 56 & 46 & 50 & 46 & 26 & 50 & 28 & 28\end{array}$ 130224B 1960 30 28 38 24 30 44 $56 \quad 54 \quad 162 \quad 172$

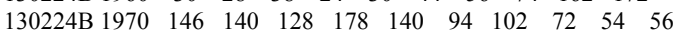

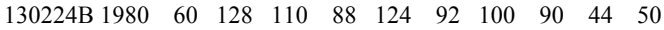
130224B 1990 $42 \begin{array}{llllllllll}44 & 24 & 36 & 16 & 26 & 22 & 20 & 26 & 18\end{array}$ 130224B 2000 $12 \quad 16 \quad 14-9999$ 130225 A $1929 \quad 56$

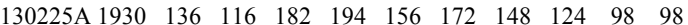

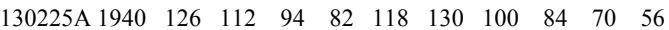

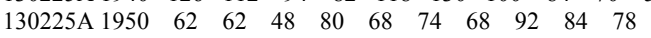




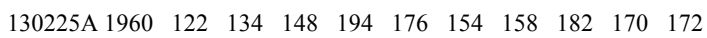
$\begin{array}{llllllllllll}130225 \text { A } 1970 & 148 & 198 & 156 & 142 & 150 & 164 & 122 & 104 & 134 & 108\end{array}$ $\begin{array}{llllllllllll}130225 \mathrm{~A} & 1980 & 128 & 114 & 138 & 110 & 66 & 110 & 152 & 134 & 78 & 104\end{array}$ $\begin{array}{llllllllllll}130225 \mathrm{~A} & 1990 & 88 & 102 & 120 & 100 & 104 & 124 & 88 & 114 & 126 & 98\end{array}$ 130225A $2000 \quad 160 \quad 154 \quad 86-9999$

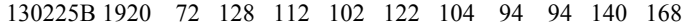

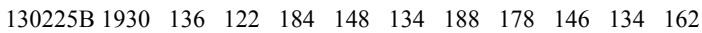

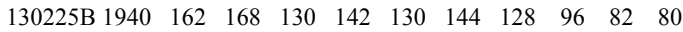

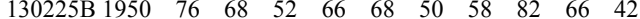

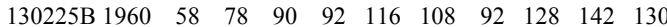

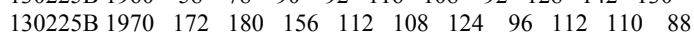

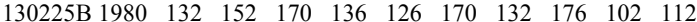
$\begin{array}{lllllllllll}130225 \mathrm{~B} 1990 & 148 & 152 & 162 & 142 & 120 & 114 & 88 & 126 & 150 & 82\end{array}$ 130225B $2000 \quad 78 \quad 64 \quad 76-9999$

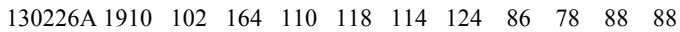

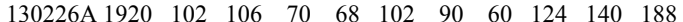
$\begin{array}{llllllllllll}130226 \mathrm{~A} & 1930 & 144 & 126 & 180 & 190 & 206 & 240 & 226 & 192 & 214 & 180\end{array}$ $\begin{array}{lllllllllll}130226 \text { A } 1940 & 168 & 156 & 172 & 120 & 114 & 98 & 126 & 102 & 104 & 128\end{array}$ 130226A 1950 $132106 \quad 48 \quad 70 \quad 50 \quad 52 \quad 72 \quad 76 \quad 66 \quad 48$

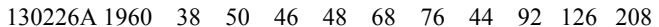

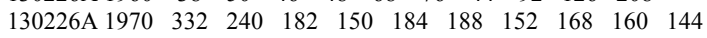
$\begin{array}{lllllllllll}130226 \text { A } 1980 & 144 & 124 & 172 & 120 & 156 & 268 & 262 & 192 & 90 & 102\end{array}$

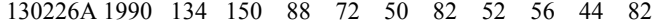
130226A $2000 \quad 48 \quad 38 \quad 48-9999$

130226B 1919190

130226B 1920 $166 \begin{array}{lllllllll}172 & 120 & 94 & 84 & 96 & 128 & 126 & 146 & 148\end{array}$ $\begin{array}{llllllllllll}130226 \mathrm{~B} & 1930 & 164 & 106 & 166 & 176 & 160 & 270 & 366 & 304 & 278 & 278\end{array}$

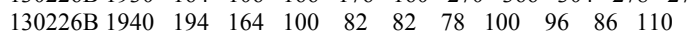

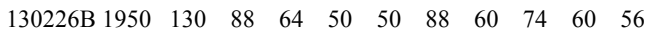

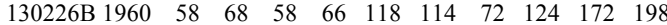

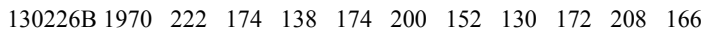

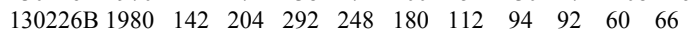

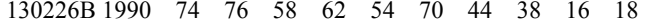
130226B $2000 \quad 28 \quad 26 \quad 48-9999$

$\begin{array}{llllllllll}130227 \mathrm{~A} 1911 & 198 & 210 & 190 & 148 & 120 & 90 & 90 & 74 & 62\end{array}$ $\begin{array}{lllllllllll}130227 \mathrm{~A} 1920 & 54 & 48 & 56 & 52 & 44 & 48 & 48 & 48 & 36 & 32\end{array}$ $\begin{array}{lllllllllll}130227 \mathrm{~A} 1930 & 78 & 108 & 110 & 72 & 86 & 92 & 82 & 130 & 128 & 106\end{array}$

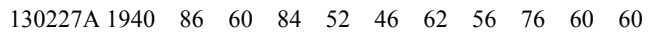
$\begin{array}{lllllllllll}130227 \mathrm{~A} 1950 & 38 & 30 & 24 & 22 & 12 & 10 & 10 & 22 & 32 & 28\end{array}$ $\begin{array}{lllllllllll}130227 \mathrm{~A} 1960 & 30 & 18 & 14 & 22 & 48 & 54 & 84 & 86 & 94 & 96\end{array}$ $\begin{array}{lllllllllll}130227 \mathrm{~A} 1970 & 74 & 68 & 94 & 58 & 46 & 60 & 36 & 40 & 30 & 34\end{array}$ $\begin{array}{lllllllllll}130227 \mathrm{~A} 1980 & 30 & 12 & 32 & 64 & 66 & 80 & 62 & 32 & 26 & 30\end{array}$ $\begin{array}{lllllllllll}130227 \mathrm{~A} 1990 & 20 & 18 & 30 & 16 & 20 & 20 & 20 & 22 & 14 & 10\end{array}$ 130227A $2000 \quad 10 \quad 6 \quad 12-9999$

130227B $1913 \quad 182 \quad 148 \quad 138 \quad 106 \quad 134 \quad 116 \quad 114$

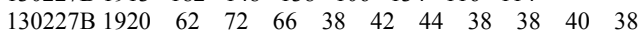
$\begin{array}{lllllllllll}130227 \mathrm{~B} 1930 & 36 & 26 & 56 & 88 & 76 & 56 & 134 & 168 & 126 & 112\end{array}$ $\begin{array}{lllllllllll}130227 \mathrm{~B} 1940 & 196 & 74 & 62 & 44 & 52 & 34 & 48 & 54 & 48 & 46\end{array}$

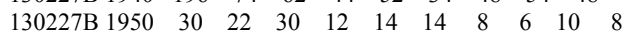
130227B 1960 $24 \begin{array}{llllllllll}14 & 14 & 14 & 22 & 42 & 72 & 90 & 70 & 68\end{array}$

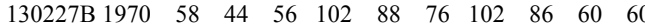
$\begin{array}{lllllllllll}130227 \mathrm{~B} 1980 & 34 & 24 & 18 & 32 & 28 & 44 & 28 & 20 & 16 & 18\end{array}$ $\begin{array}{lllllllllll}130227 \mathrm{~B} 1990 & 22 & 10 & 16 & 8 & 12 & 10 & 10 & 20 & 8 & 8\end{array}$

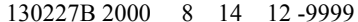

130228A $1963 \quad 72 \quad 364 \quad 450 \quad 492 \quad 310 \quad 376 \quad 342$

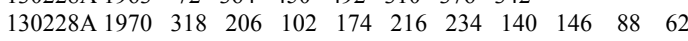

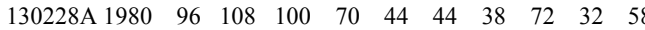

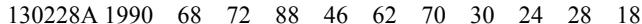
130228A $2000 \quad 38 \quad 22 \quad 46-9999$

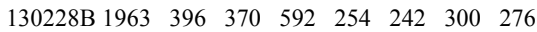

$\begin{array}{lllllllllll}130228 \mathrm{~B} 1970 & 246 & 120 & 208 & 230 & 226 & 232 & 212 & 140 & 122 & 168\end{array}$

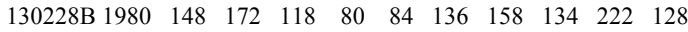

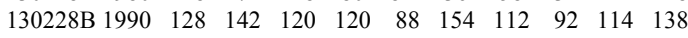
130228B $2000 \quad 84 \quad 50 \quad 66-9999$

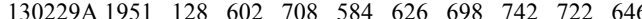
$\begin{array}{lllllllllll}130229 \mathrm{~A} 1960 & 744 & 700 & 544 & 470 & 424 & 318 & 370 & 486 & 498 & 650\end{array}$

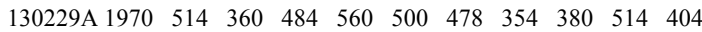

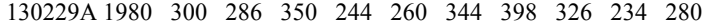

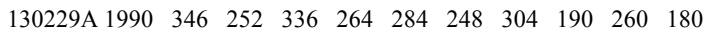
130229A $2000 \quad 302 \quad 336 \quad 190-9999$

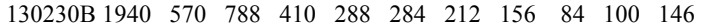

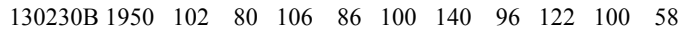

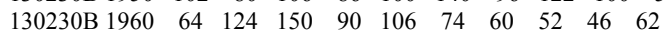
130230B 1970 $74 \begin{array}{llllllllll}70 & 58 & 80 & 54 & 56 & 56 & 36 & 70 & 74\end{array}$

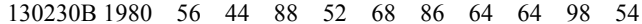
130230B 1990 $66 \begin{array}{llllllllll}42 & 64 & 46 & 48 & 60 & 38 & 30 & 42 & 52\end{array}$ $\begin{array}{lllll}130230 \mathrm{~B} 2000 & 34 & 38 & 28 & -9999\end{array}$

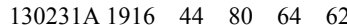

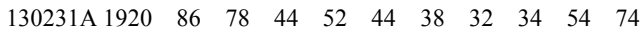

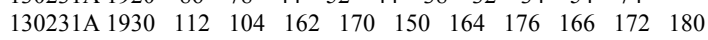

$\begin{array}{lllllllllll}130231 \mathrm{~A} 1940 & 168 & 142 & 142 & 152 & 140 & 164 & 104 & 82 & 110 & 88\end{array}$ $\begin{array}{lllllllllll}130231 \mathrm{~A} 1950 & 178 & 192 & 112 & 148 & 132 & 130 & 122 & 156 & 126 & 96\end{array}$

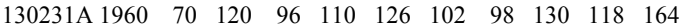
$\begin{array}{llllllllllll}130231 \mathrm{~A} & 1970 & 146 & 144 & 116 & 106 & 124 & 126 & 112 & 102 & 136 & 104\end{array}$

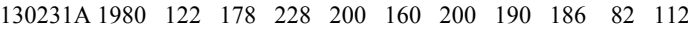

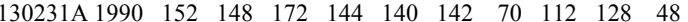
130231A $2000 \quad 80 \quad 104 \quad 74-9999$ 130231B $1934 \quad 176 \quad 228 \quad 248 \quad 198 \quad 208 \quad 186$

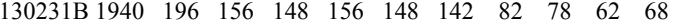
130231B 1950 $80 \begin{array}{llllllllll}70 & 34 & 48 & 34 & 72 & 90 & 92 & 88 & 54\end{array}$

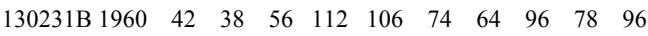

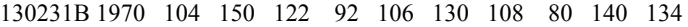
$\begin{array}{lllllllllll}130231 \mathrm{~B} 1980 & 190 & 188 & 200 & 160 & 150 & 184 & 180 & 134 & 72 & 90\end{array}$

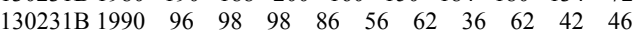
130231B $2000 \quad 46 \quad 62 \quad 56-9999$

$130232 \mathrm{~A} 1958 \quad 66 \quad 64$

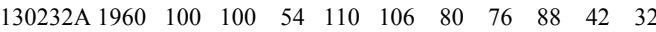
$\begin{array}{lllllllllll}130232 \mathrm{~A} 1970 & 32 & 48 & 40 & 24 & 36 & 52 & 72 & 46 & 34 & 40\end{array}$ $\begin{array}{lllllllllll}130232 \mathrm{~A} 1980 & 52 & 96 & 54 & 108 & 86 & 58 & 38 & 24 & 14 & 8\end{array}$

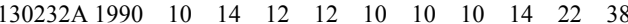
130232A $2000 \quad 74 \quad 98 \quad 40-9999$

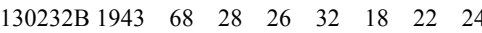

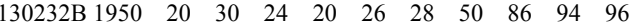

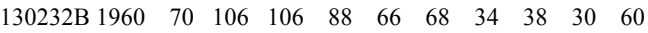

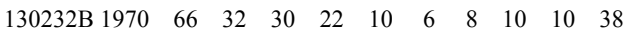

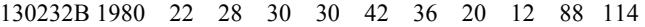
$\begin{array}{lllllllllll}130232 \mathrm{~B} 1990 & 140 & 74 & 60 & 38 & 32 & 20 & 36 & 6 & 10 & 38\end{array}$ 130232B $2000 \quad 36 \quad 52 \quad 60-9999$

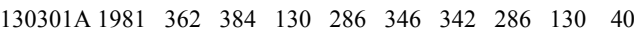
$\begin{array}{lllllllllll}130301 \mathrm{~A} 1990 & 172 & 164 & 154 & 170 & 154 & 80 & 76 & 72 & 38 & 16\end{array}$ 130301A $2000 \quad 66 \quad 40 \quad 38-9999$

130301B $1974 \quad 88 \quad 88 \quad 192 \quad 122 \quad 396 \quad 394$

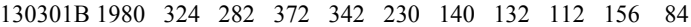

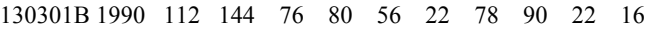
130301B $2000 \quad 20 \quad 36 \quad 54-9999$

130302A $1948 \quad 322 \quad 280$

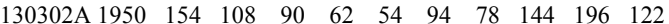

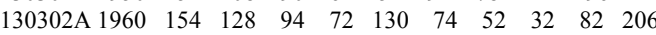
$\begin{array}{lllllllllll}130302 \mathrm{~A} 1970 & 204 & 258 & 232 & 166 & 104 & 170 & 132 & 164 & 190 & 84\end{array}$

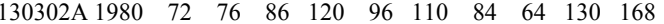

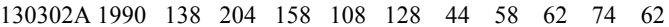
130302A $2000 \quad 66 \quad 30 \quad 40-9999$

$130302 \mathrm{~B} 194996$

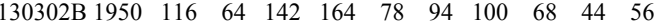

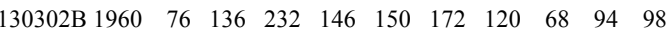
$\begin{array}{llllllllllll}130302 \mathrm{~B} & 1970 & 120 & 130 & 100 & 118 & 74 & 144 & 222 & 168 & 204 & 148\end{array}$

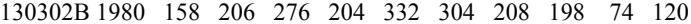
$\begin{array}{lllllllllll}130302 \mathrm{~B} 1990 & 90 & 108 & 92 & 96 & 106 & 88 & 62 & 68 & 148 & 90\end{array}$ 130302B $2000 \quad 94 \quad 190 \quad 64-9999$

130303A 1949552

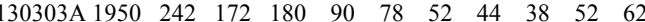

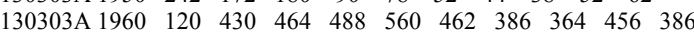

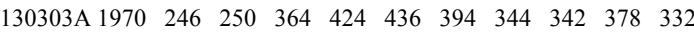

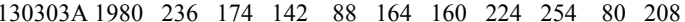

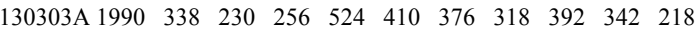
130303A $2000 \quad 236 \quad 194 \quad 42-9999$

130303B $1928 \quad 190 \quad 144$

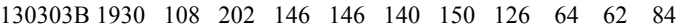

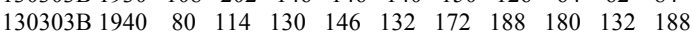

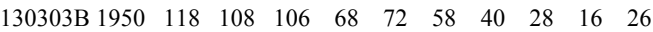

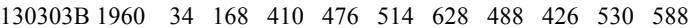
$\begin{array}{llllllllllll}130303 \mathrm{~B} & 1970 & 514 & 364 & 406 & 324 & 330 & 374 & 382 & 296 & 264 & 344\end{array}$

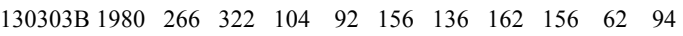

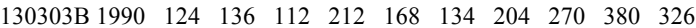
130303B 2000 $410 \quad 436 \quad 150-9999$ 130304A $1966 \quad 720 \quad 506 \quad 436 \quad 434$

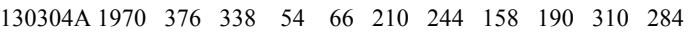

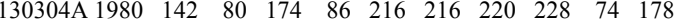
130304A 1990 $278 \begin{array}{llllllllll}184 & 122 & 314 & 208 & 236 & 226 & 228 & 274 & 160\end{array}$ 130304A $2000 \quad 308 \quad 256 \quad 66-9999$

130304B $1964 \quad 290 \quad 550 \quad 458 \quad 296 \quad 298 \quad 336$

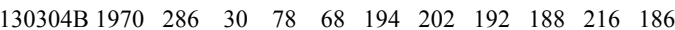
$\begin{array}{lllllllllll}130304 \mathrm{~B} 1980 & 118 & 92 & 104 & 66 & 204 & 240 & 200 & 178 & 58 & 132\end{array}$ 130304B 1990 $268 \begin{array}{llllllllll}202 & 134 & 348 & 300 & 342 & 326 & 380 & 394 & 286\end{array}$ 130304B 2000 $408 \quad 358 \quad 80-9999$

130305A $1958 \quad 64 \quad 36$

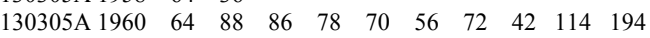

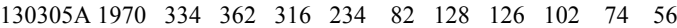

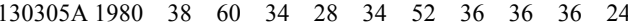
$\begin{array}{lllllllllll}130305 \mathrm{~A} 1990 & 20 & 32 & 18 & 28 & 24 & 20 & 22 & 38 & 20 & 28\end{array}$ 
130305A $2000 \quad 36 \quad 64 \quad 32-9999$

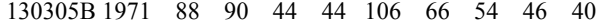

$\begin{array}{lllllllllll}130305 \mathrm{~B} 1980 & 46 & 40 & 104 & 170 & 250 & 296 & 344 & 292 & 172 & 168\end{array}$

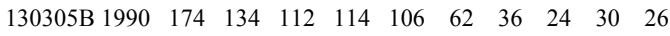

130305B 2000 $20 \quad 24 \quad 62-9999$

130306A $1927 \quad 74 \quad 38 \quad 38$

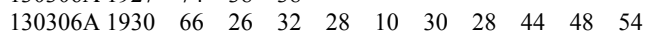

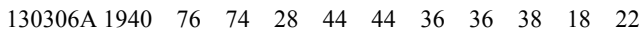

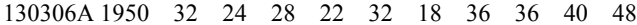

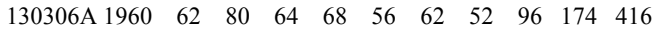

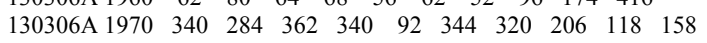

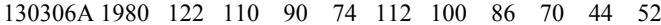

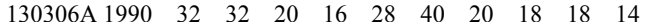

130306A $2000 \quad 10 \quad 12 \quad 8-9999$

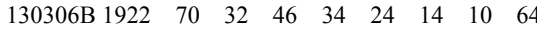

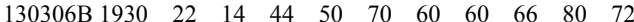

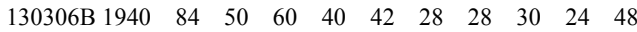

$\begin{array}{lllllllllll}130306 \mathrm{~B} 1950 & 22 & 74 & 30 & 38 & 66 & 66 & 72 & 74 & 72 & 58\end{array}$

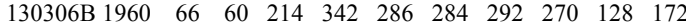

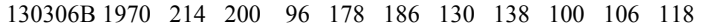

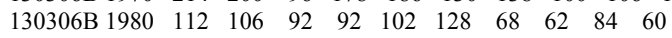

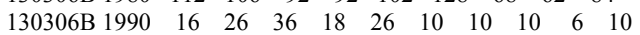

130306B 2000 $10 \quad 12 \quad 10-9999$

130307A $1945 \quad 110 \quad 66 \quad 18 \quad 32 \quad 46$

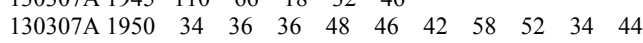

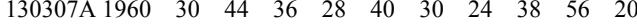

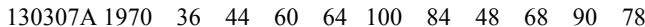

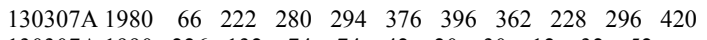

130307A 1990 $226 \begin{array}{lllllllll}132 & 74 & 74 & 42 & 20 & 30 & 12 & 32 & 52\end{array}$

130307A $2000 \quad 34 \quad 48 \quad 30-9999$

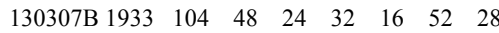

130307B 1940 $30 \begin{array}{llllllllll}12 & 50 & 28 & 40 & 32 & 36 & 36 & 10 & 18\end{array}$

130307B 1950 $24 \begin{array}{llllllllll}20 & 26 & 20 & 44 & 22 & 22 & 20 & 18 & 34\end{array}$

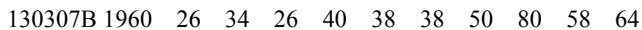

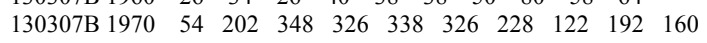

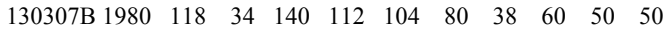

$\begin{array}{lllllllllll}130307 \mathrm{~B} 1990 & 24 & 28 & 32 & 14 & 52 & 16 & 18 & 16 & 16 & 14\end{array}$

130307B $2000 \quad 4 \quad 14 \quad 18-9999$

130308A $1967 \quad 300 \quad 404 \quad 378$

$\begin{array}{lllllllllll}130308 \text { A } 1970 & 404 & 346 & 224 & 158 & 302 & 338 & 370 & 426 & 372 & 272\end{array}$

$\begin{array}{lllllllllll}130308 \text { A } 1980 & 362 & 308 & 304 & 246 & 190 & 220 & 178 & 104 & 298 & 170\end{array}$

$\begin{array}{lllllllllll}130308 \text { A } 1990 & 102 & 134 & 122 & 194 & 186 & 150 & 200 & 216 & 276 & 154\end{array}$

130308A $2000 \quad 290 \quad 314 \quad 78-9999$

130308B $1987 \quad 110 \quad 568 \quad 660$

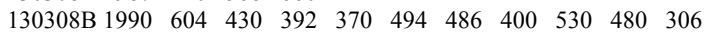

130308B $2000 \quad 262 \quad 430 \quad 254-9999$

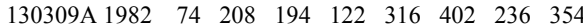

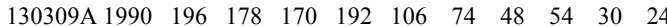

130309A $2000 \quad 64 \quad 90 \quad 88-9999$

130309B $1983 \quad 258 \quad 88 \quad 332 \quad 372 \quad 374 \quad 288 \quad 244$

130309B 1990 $142 \begin{array}{llllllllll}134 & 120 & 172 & 106 & 76 & 46 & 32 & 34 & 58\end{array}$

130309B $2000 \quad 66 \quad 50 \quad 112-9999$

130310A $1968 \quad 260 \quad 376$

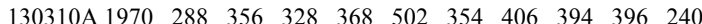

$\begin{array}{lllllllllll}130310 \text { A } 1980 & 320 & 330 & 374 & 254 & 230 & 406 & 398 & 212 & 238 & 108\end{array}$

$\begin{array}{llllllllllll}130310 \text { A } 1990 & 192 & 248 & 268 & 210 & 174 & 168 & 124 & 176 & 92 & 200\end{array}$

130310A $2000 \quad 406 \quad 392 \quad 182-9999$

130310B 1969340

$\begin{array}{lllllllllll}130310 \mathrm{~B} 1970 & 308 & 282 & 286 & 432 & 444 & 398 & 296 & 260 & 270 & 246\end{array}$

$\begin{array}{lllllllllll}130310 \mathrm{~B} 1980 & 348 & 382 & 354 & 318 & 302 & 514 & 566 & 474 & 440 & 218\end{array}$

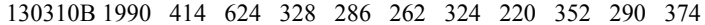

130310B $2000 \quad 430 \quad 330 \quad 232-9999$

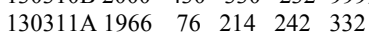

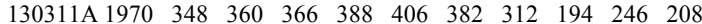

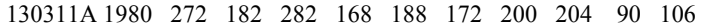

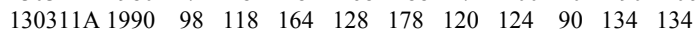

130311A $2000 \quad 218 \quad 184 \quad 86-9999$

130311B 1969326

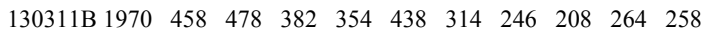

$\begin{array}{lllllllllll}130311 \mathrm{~B} 1980 & 278 & 318 & 224 & 302 & 298 & 272 & 406 & 252 & 132 & 176\end{array}$

$\begin{array}{lllllllllll}130311 \mathrm{~B} 1990 & 244 & 208 & 276 & 244 & 240 & 340 & 246 & 292 & 284 & 186\end{array}$

130311B $2000 \quad 318 \quad 318 \quad 124-9999$

130312A $1966 \quad 62 \quad 482 \quad 476 \quad 482$

$\begin{array}{lllllllllll}130312 \text { A } 1970 & 398 & 330 & 170 & 234 & 182 & 262 & 180 & 120 & 154 & 180\end{array}$

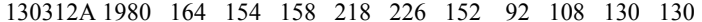

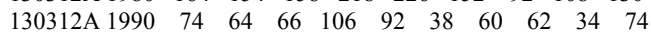

130312A $2000 \quad 84 \quad 86 \quad 50-9999$

130312B $1963 \quad 86 \quad 176 \quad 350 \quad 338 \quad 368 \quad 394 \quad 452$

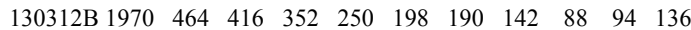

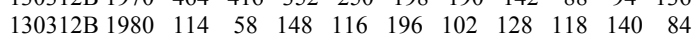

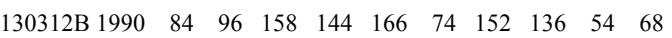

130312B $2000 \quad 98 \quad 72 \quad 78-9999$

130313A $1977 \quad 340 \quad 430 \quad 430$

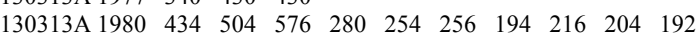

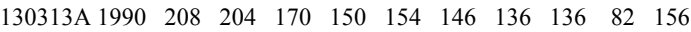

130313A $2000 \quad 152 \quad 142 \quad 146-9999$

130313B 1979340

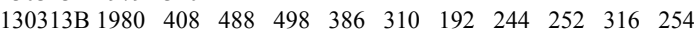

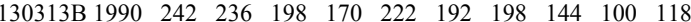
130313B 2000 $158 \quad 106 \quad 128-9999$

130314A 1951 $28 \quad 44 \quad 16 \quad 50 \quad 72 \quad 24 \quad 30 \quad 204 \quad 52$

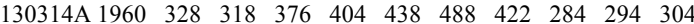

$\begin{array}{lllllllllll}130314 \mathrm{~A} 1970 & 86 & 290 & 262 & 198 & 216 & 92 & 134 & 160 & 76 & 128\end{array}$

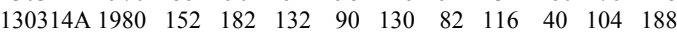

$\begin{array}{lllllllllll}130314 \mathrm{~A} 1990 & 88 & 74 & 96 & 48 & 48 & 50 & 66 & 26 & 60 & 56\end{array}$

130314A $2000 \quad 52 \quad 84 \quad 58-9999$

130314B $1937 \quad 122 \quad 88 \quad 78$

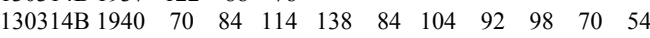

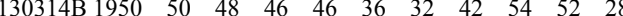

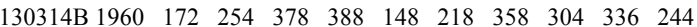

$\begin{array}{llllllllllll}130314 \mathrm{~B} 1970 & 254 & 336 & 258 & 188 & 148 & 160 & 118 & 74 & 78 & 88\end{array}$

$\begin{array}{lllllllllll}130314 \mathrm{~B} 1980 & 156 & 154 & 134 & 132 & 102 & 58 & 52 & 100 & 86 & 88\end{array}$

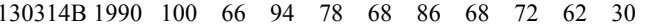

130314B $2000 \quad 58 \quad 40 \quad 86-9999$

130315A $1934 \quad 60 \quad 144 \quad 268 \quad 230 \quad 230 \quad 200$

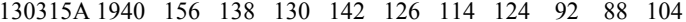

$\begin{array}{lllllllllll}130315 \mathrm{~A} 1950 & 76 & 74 & 68 & 70 & 88 & 42 & 48 & 70 & 74 & 64\end{array}$

$\begin{array}{lllllllllll}130315 \mathrm{~A} 1960 & 84 & 72 & 36 & 92 & 454 & 296 & 218 & 652 & 634 & 554\end{array}$

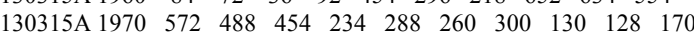

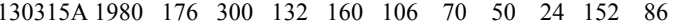

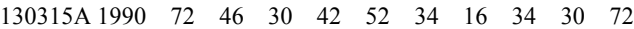

130315A $2000 \quad 52 \quad 42 \quad 74-9999$

130315B $1934 \quad 70 \quad 178 \quad 198 \quad 158 \quad 138 \quad 134$

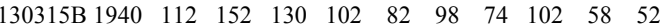

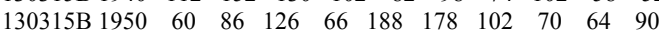

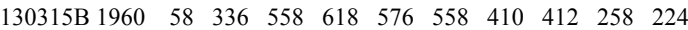

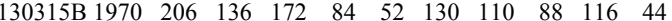

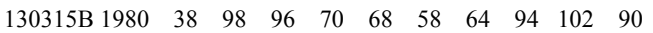

$\begin{array}{lllllllllll}130315 \mathrm{~B} 1990 & 56 & 122 & 14 & 46 & 88 & 84 & 82 & 36 & 74 & 66\end{array}$

130315B $2000 \quad 34 \quad 54 \quad 48-9999$

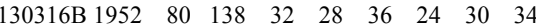

$\begin{array}{lllllllllll}130316 \mathrm{~B} 1960 & 50 & 28 & 48 & 78 & 44 & 64 & 50 & 96 & 224 & 394\end{array}$

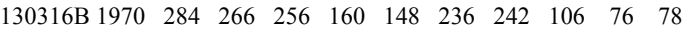

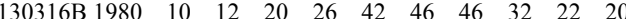

$\begin{array}{lllllllllll}130316 \mathrm{~B} 1990 & 20 & 36 & 26 & 48 & 22 & 24 & 18 & 26 & 24 & 40\end{array}$

130316B $2000 \quad 36 \quad 18 \quad 26-9999$

130317B $1948 \quad 156 \quad 98$

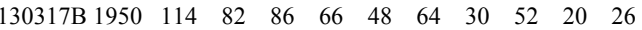

$\begin{array}{llllllllllll}130317 \mathrm{~B} 1960 & 36 & 30 & 44 & 38 & 110 & 378 & 696 & 488 & 302 & 344\end{array}$

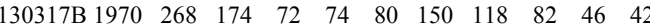

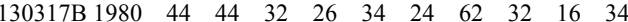

$\begin{array}{lllllllllll}130317 \mathrm{~B} 1990 & 58 & 40 & 40 & 38 & 10 & 26 & 28 & 24 & 48 & 26\end{array}$

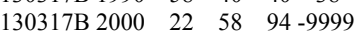

$\begin{array}{llllll}130318 A & 1926 & 138 & 152 & 104 & 76\end{array}$

$\begin{array}{lllllllllll}130318 \text { A } 1930 & 16 & 26 & 18 & 8 & 20 & 14 & 38 & 34 & 92 & 134\end{array}$

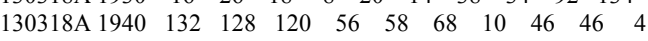

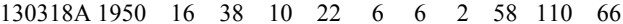

$\begin{array}{lllllllllll}130318 \mathrm{~A} 1960 & 82 & 62 & 74 & 44 & 220 & 414 & 324 & 304 & 280 & 196\end{array}$

$\begin{array}{lllllllllll}130318 \text { A } 1970 & 214 & 170 & 110 & 114 & 160 & 162 & 104 & 102 & 82 & 58\end{array}$

$\begin{array}{lllllllllll}130318 \text { A } 1980 & 120 & 190 & 148 & 152 & 152 & 170 & 78 & 88 & 70 & 56\end{array}$

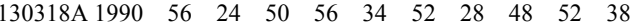

130318A $2000 \quad 52 \quad 68 \quad 50-9999$

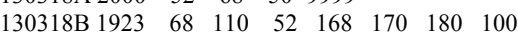

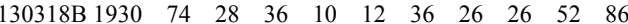

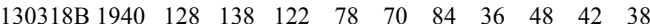

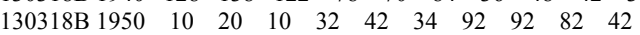

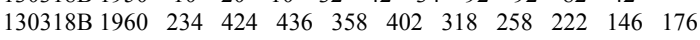

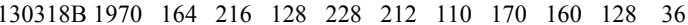

130318B 1980 $202 \begin{array}{llllllllll}154 & 78 & 100 & 66 & 78 & 66 & 56 & 64 & 52\end{array}$

$\begin{array}{lllllllllll}130318 \mathrm{~B} 1990 & 30 & 14 & 14 & 20 & 8 & 8 & 8 & 28 & 14 & 16\end{array}$

130318B $2000 \quad 14 \quad 12 \quad 28-9999$

$\begin{array}{llllllll}130319 \text { A } 1943 & 314 & 222 & 300 & 274 & 246 & 328 & 302\end{array}$

$\begin{array}{llllllllllll}130319 \mathrm{~A} 1950 & 238 & 254 & 254 & 258 & 184 & 214 & 202 & 272 & 284 & 232\end{array}$

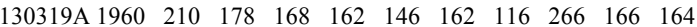

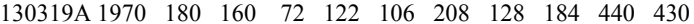

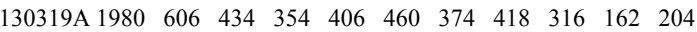

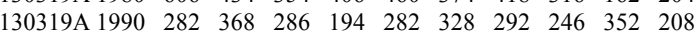
130319A 2000 $122 \quad 264 \quad 54-9999$

130319B $1948 \quad 340 \quad 450$

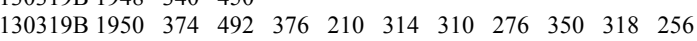


$\begin{array}{lllllllllll}\text { 130319B } 1960 & 244 & 312 & 312 & 122 & 224 & 186 & 94 & 138 & 100 & 122\end{array}$ 130319B 1970 $212 \quad 210298 \begin{array}{lllllllll}376 & 354 & 466 & 196 & 134 & 200 & 102\end{array}$

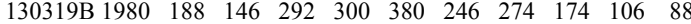

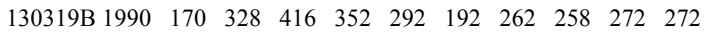
130319B 2000 $122 \quad 254 \quad 256-9999$

$\begin{array}{lllllll}130401 \mathrm{~A} & 1955 & 108 & 186 & 172 & 118 & 136\end{array}$

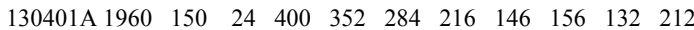
$\begin{array}{lllllllllll}130401 \mathrm{~A} 1970 & 236 & 216 & 294 & 280 & 394 & 206 & 168 & 86 & 84 & 134\end{array}$ $\begin{array}{lllllllllll}130401 \mathrm{~A} 1980 & 232 & 246 & 230 & 152 & 136 & 182 & 152 & 144 & 72 & 130\end{array}$

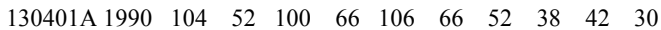
130401A $2000 \quad 56 \quad 54 \quad 48-9999$

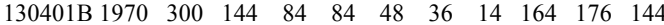
$\begin{array}{lllllllllll}130401 \mathrm{~B} 1980 & 202 & 168 & 90 & 126 & 206 & 118 & 82 & 60 & 156 & 140\end{array}$ $\begin{array}{lllllllllll}130401 \mathrm{~B} 1990 & 58 & 166 & 152 & 146 & 36 & 54 & 58 & 76 & 46 & 132\end{array}$ 130401B 2000 $62 \quad 72 \quad 90-9999$

130402 A $1937 \quad 78 \quad 94 \quad 42$

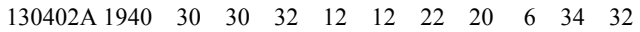
$\begin{array}{lllllllllll}130402 \mathrm{~A} 1950 & 10 & 38 & 56 & 98 & 38 & 122 & 114 & 96 & 104 & 92\end{array}$

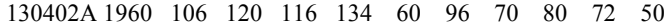

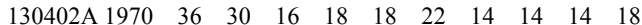

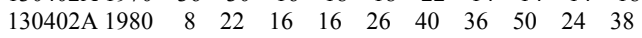
$\begin{array}{lllllllllll}130402 \mathrm{~A} 1990 & 38 & 26 & 30 & 42 & 32 & 40 & 40 & 26 & 10 & 8\end{array}$ 130402A $2000 \quad 10 \quad 12 \quad 28-9999$

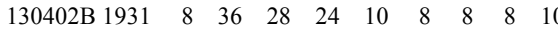

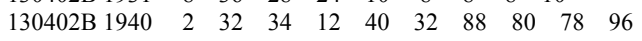
$\begin{array}{lllllllllll}130402 \mathrm{~B} 1950 & 90 & 92 & 82 & 100 & 82 & 120 & 128 & 52 & 72 & 32\end{array}$

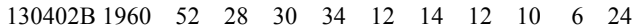

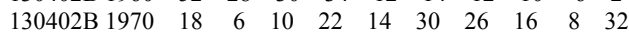

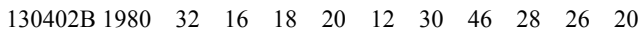

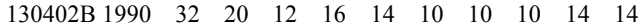
130402B 2000 $12 \quad 26 \quad 10-9999$

$\begin{array}{lllllllllll}\text { 130403A } 1970 & 86 & 74 & 72 & 86 & 84 & 46 & 80 & 102 & 102 & 130\end{array}$

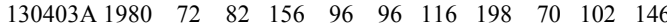
$\begin{array}{llllllllllll}130403 \mathrm{~A} & 1990 & 80 & 114 & 150 & 136 & 114 & 92 & 86 & 96 & 80 & 70\end{array}$ 130403A $2000 \quad 156 \quad 110 \quad 76-9999$

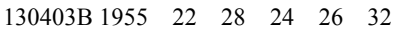

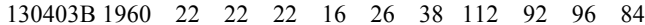
$\begin{array}{lllllllllll}130403 \mathrm{~B} 1970 & 64 & 58 & 28 & 54 & 58 & 78 & 80 & 44 & 50 & 158\end{array}$

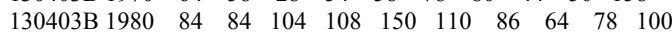

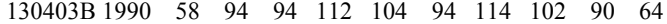
130403B $2000 \quad 50 \quad 78 \quad 84-9999$

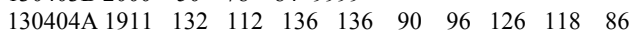

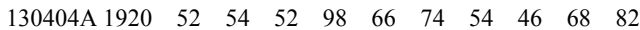
$\begin{array}{lllllllllll}130404 \mathrm{~A} 1930 & 46 & 78 & 38 & 62 & 68 & 98 & 152 & 212 & 204 & 160\end{array}$

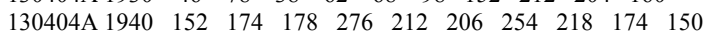
$\begin{array}{lllllllllll}130404 \mathrm{~A} 1950 & 154 & 138 & 88 & 100 & 104 & 82 & 68 & 100 & 120 & 74\end{array}$

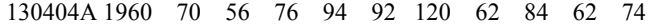
$\begin{array}{llllllllllll}130404 \mathrm{~A} & 1970 & 114 & 94 & 88 & 102 & 128 & 122 & 102 & 92 & 44 & 68\end{array}$ $\begin{array}{lllllllllll}130404 \mathrm{~A} 1980 & 52 & 144 & 106 & 92 & 72 & 54 & 64 & 54 & 54 & 32\end{array}$ $\begin{array}{lllllllllll}130404 \mathrm{~A} 1990 & 44 & 42 & 34 & 42 & 56 & 32 & 36 & 32 & 28 & 26\end{array}$ 130404A $2000 \quad 28 \quad 38 \quad 40-9999$

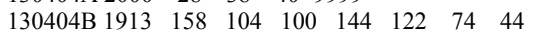

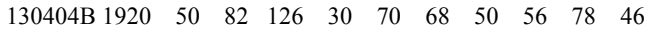
130404B 1930 $60 \begin{array}{llllllllll}34 & 84 & 82 & 114 & 152 & 184 & 140 & 126 & 132\end{array}$

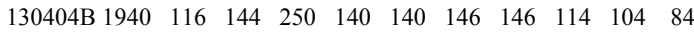

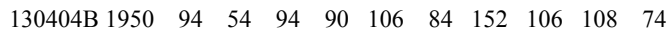
130404B 1960 $64 \begin{array}{llllllllll}88 & 60 & 66 & 98 & 80 & 68 & 46 & 54 & 80\end{array}$ 130404B 1970 $70 \begin{array}{llllllllll}78 & 82 & 76 & 60 & 70 & 74 & 34 & 44 & 40\end{array}$ 130404B 1980 $58 \begin{array}{llllllllll}80 & 60 & 80 & 62 & 76 & 68 & 68 & 48 & 68\end{array}$ $\begin{array}{lllllllllll}130404 \mathrm{~B} 1990 & 48 & 48 & 42 & 74 & 36 & 42 & 18 & 16 & 16 & 18\end{array}$ 130404B 2000 $16 \quad 24 \quad 28-9999$

130405A $1937 \quad 26 \quad 28 \quad 16$

$\begin{array}{lllllllllll}130405 \mathrm{~A} 1940 & 28 & 14 & 16 & 22 & 62 & 68 & 66 & 82 & 78 & 90\end{array}$ 130405A 1950 $96 \begin{array}{llllllllll}94 & 102 & 66 & 96 & 78 & 96 & 54 & 18 & 28\end{array}$

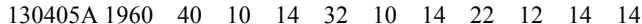
$\begin{array}{lllllllllll}130405 \mathrm{~A} 1970 & 12 & 10 & 82 & 46 & 86 & 32 & 24 & 22 & 32 & 42\end{array}$ $\begin{array}{lllllllllll}130405 \mathrm{~A} 1980 & 52 & 46 & 64 & 76 & 78 & 36 & 22 & 132 & 76 & 80\end{array}$ $\begin{array}{lllllllllll}130405 \mathrm{~A} 1990 & 54 & 50 & 60 & 28 & 20 & 50 & 18 & 16 & 28 & 108\end{array}$ 130405A $2000 \quad 36 \quad 32 \quad 28-9999$

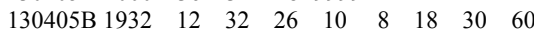

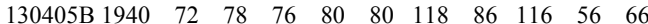
$\begin{array}{lllllllllll}130405 \mathrm{~B} 1950 & 80 & 70 & 56 & 46 & 38 & 28 & 14 & 14 & 12 & 10\end{array}$

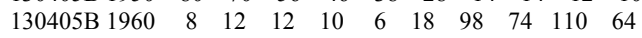

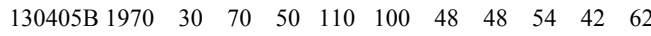

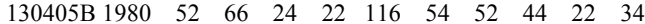
$\begin{array}{lllllllllll}130405 \mathrm{~B} 1990 & 40 & 18 & 38 & 28 & 28 & 90 & 44 & 22 & 38 & 36\end{array}$

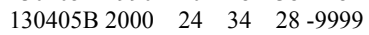

$\begin{array}{llll}130406 \text { A } 1937 & 84 & 108 & 108\end{array}$

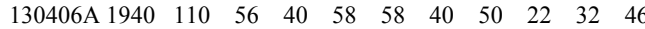

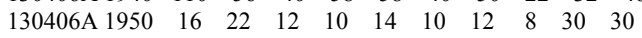

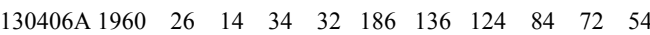

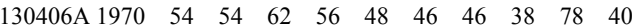

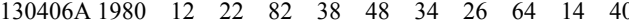

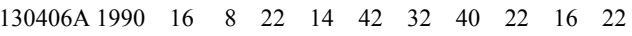
130406A $2000 \quad 22 \quad 36 \quad 28-9999$

130406B $1927 \quad 100 \quad 154 \quad 170$

130406B 1930 $68 \begin{array}{llllllllll}36 & 14 & 38 & 22 & 26 & 68 & 54 & 64 & 60\end{array}$ $\begin{array}{llllllllllll}130406 \mathrm{~B} 1940 & 14 & 64 & 74 & 20 & 54 & 44 & 30 & 16 & 12 & 12\end{array}$ 130406B 1950 $34 \begin{array}{llllllllll}12 & 14 & 6 & 10 & 44 & 30 & 16 & 14 & 8\end{array}$

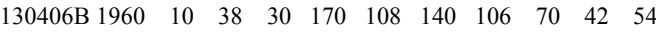

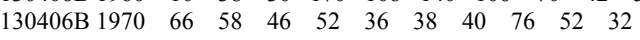
130406B 1980 $36 \begin{array}{llllllllll}118 & 66 & 78 & 48 & 18 & 18 & 70 & 24 & 28\end{array}$

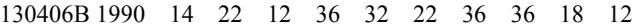
$\begin{array}{lllll}130406 \mathrm{~B} 2000 & 14 & 24 & 12 & -9999\end{array}$ 130407A $1937 \quad 54 \quad 88 \quad 80$

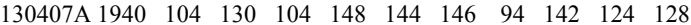
130407A 1950 $96 \begin{array}{llllllllll} & 96 & 86 & 80 & 70 & 66 & 52 & 56 & 92 & 130\end{array}$

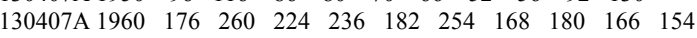
$\begin{array}{lllllllllll}\text { 130407A } 1970 & 174 & 152 & 118 & 106 & 118 & 74 & 78 & 122 & 90 & 78\end{array}$

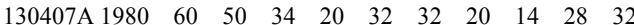
$\begin{array}{lllllllllll}130407 \mathrm{~A} 1990 & 30 & 20 & 30 & 32 & 36 & 32 & 22 & 24 & 30 & 28\end{array}$ 130407A $2000 \quad 30 \quad 26 \quad 36-9999$

130407B $1928 \quad 96 \quad 254$

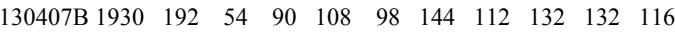

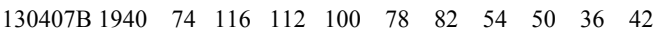

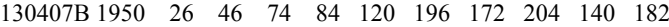

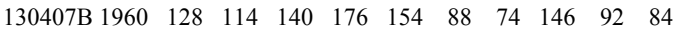
$\begin{array}{lllllllllll}130407 \mathrm{~B} 1970 & 92 & 52 & 52 & 42 & 44 & 30 & 22 & 26 & 28 & 50\end{array}$ $\begin{array}{lllllllllll}130407 \mathrm{~B} 1980 & 40 & 48 & 52 & 18 & 28 & 30 & 32 & 36 & 24 & 24\end{array}$

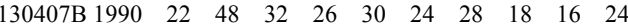
130407B 2000 $30 \quad 20 \quad 46-9999$

130408A $1954 \quad 88 \quad 122 \quad 100 \quad 212 \quad 234 \quad 66$

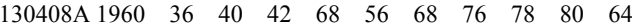

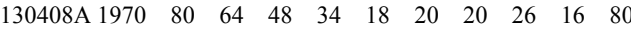

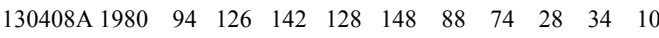

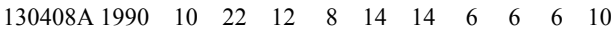
130408 A $2000 \quad 8 \quad 28 \quad 42-9999$

130408B 1950 $68 \begin{array}{llllllllll} & 68 & 176 & 184 & 48 & 30 & 26 & 40 & 48 & 62\end{array}$ $\begin{array}{lllllllllll}130408 \mathrm{~B} 1960 & 70 & 100 & 60 & 34 & 42 & 36 & 46 & 12 & 22 & 12\end{array}$

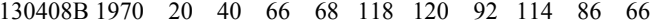
130408B 1980 $60 \begin{array}{llllllllll}70 & 30 & 10 & 32 & 34 & 24 & 8 & 6 & 12\end{array}$

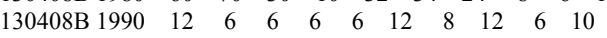
130408B 2000 $12 \quad 10 \quad 12-9999$

130409A $1935 \quad 56 \quad 224 \quad 40 \quad 36 \quad 30$

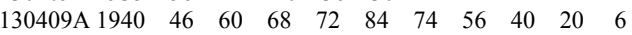
$\begin{array}{lllllllllll}130409 \text { A } 1950 & 10 & 10 & 22 & 42 & 24 & 26 & 64 & 52 & 62 & 78\end{array}$

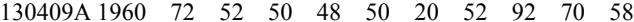

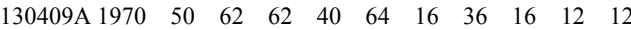
130409A $1980 \quad 8 \quad 4 \quad \begin{array}{lllllllll} & 8 & 6 & 4 & 10 & 6 & 24 & 34 & 74\end{array}$ $\begin{array}{lllllllllll}130409 \text { A } 1990 & 54 & 54 & 86 & 60 & 26 & 58 & 14 & 38 & 18 & 22\end{array}$ 130409A $2000 \quad 12 \quad 10 \quad 26-9999$

130409B $1926 \quad 134 \quad 138 \quad 108 \quad 172$

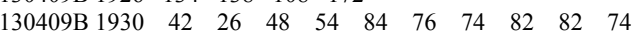

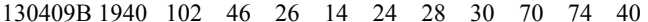

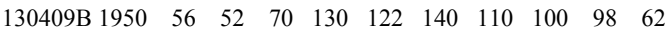

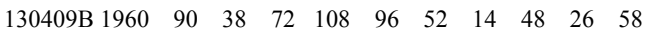

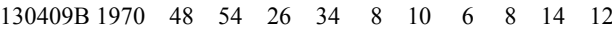

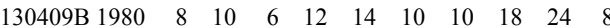

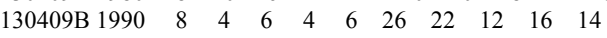
130409B $2000 \quad 8 \quad 18 \quad 16-9999$

130410A $1937 \quad 390 \quad 724 \quad 674$

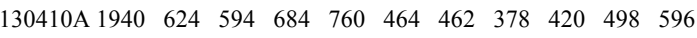
$\begin{array}{lllllllllll}130410 \text { A } 1950 & 394 & 320 & 364 & 426 & 342 & 364 & 378 & 480 & 330 & 214\end{array}$

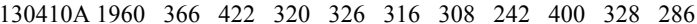

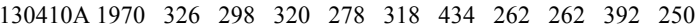
$\begin{array}{lllllllllll}130410 \text { A } 1980 & 278 & 312 & 478 & 380 & 302 & 318 & 380 & 264 & 172 & 194\end{array}$ $\begin{array}{lllllllllll}130410 \mathrm{~A} 1990 & 192 & 182 & 304 & 212 & 208 & 220 & 256 & 198 & 194 & 122\end{array}$ 130410A $2000 \quad 176 \quad 168 \quad 148-9999$

130410B $1935 \quad 48 \quad 548 \quad 724 \quad 634 \quad 552$

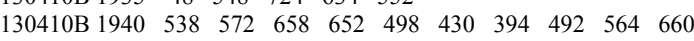

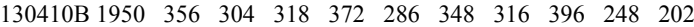
130410B 1960 $26 \begin{array}{llllllllll}436 & 288 & 318 & 282 & 206 & 168 & 272 & 274 & 242\end{array}$ $\begin{array}{llllllllllll}130410 \mathrm{~B} & 1970 & 366 & 278 & 314 & 268 & 396 & 408 & 216 & 164 & 362 & 208\end{array}$ 130410B 1980 $290 \begin{array}{llllllllll}264 & 400 & 282 & 192 & 188 & 320 & 212 & 132 & 128\end{array}$

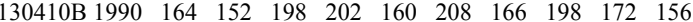
130410B 2000 $198 \quad 178 \quad 146-9999$

$\begin{array}{lllllllllll}130411 \mathrm{~A} 1960 & 36 & 32 & 28 & 42 & 58 & 70 & 66 & 74 & 50 & 66\end{array}$

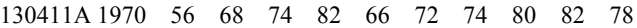

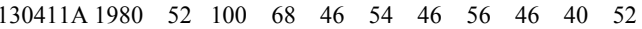

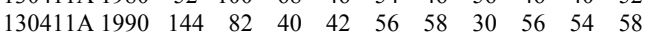


130411A $2000 \quad 82 \quad 88 \quad 36-9999$

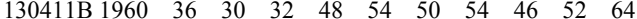
$\begin{array}{lllllllllll}130411 \mathrm{~B} 1970 & 66 & 70 & 46 & 38 & 46 & 64 & 64 & 46 & 52 & 40\end{array}$ $\begin{array}{lllllllllll}130411 \mathrm{~B} 1980 & 58 & 52 & 40 & 40 & 28 & 52 & 40 & 32 & 30 & 36\end{array}$

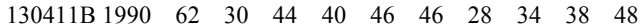
130411B 2000 $\quad 88 \quad 80 \quad 36-9999$

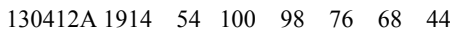

$\begin{array}{lllllllllll}130412 \mathrm{~A} 1920 & 38 & 32 & 30 & 26 & 24 & 32 & 28 & 28 & 18 & 40\end{array}$ $\begin{array}{lllllllllll}130412 \mathrm{~A} 1930 & 44 & 18 & 50 & 38 & 68 & 74 & 92 & 96 & 92 & 70\end{array}$ $\begin{array}{lllllllllll}130412 \mathrm{~A} 1940 & 42 & 58 & 84 & 76 & 74 & 78 & 84 & 78 & 86 & 82\end{array}$ $\begin{array}{lllllllllll}130412 \text { A } 1950 & 94 & 38 & 62 & 82 & 62 & 72 & 82 & 42 & 78 & 46\end{array}$ $\begin{array}{lllllllllll}130412 \text { A } 1960 & 58 & 68 & 68 & 60 & 74 & 80 & 68 & 70 & 48 & 60\end{array}$

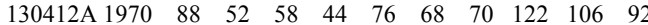

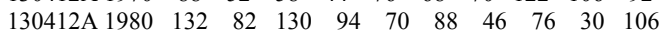
$\begin{array}{llllllllllll}130412 \mathrm{~A} 1990 & 110 & 60 & 178 & 102 & 140 & 64 & 44 & 38 & 90 & 46\end{array}$ 130412A $2000 \quad 106 \quad 110 \quad 48-9999$

130412B $1917 \quad 104 \quad 84 \quad 84$

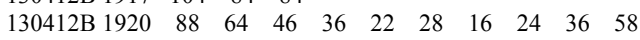

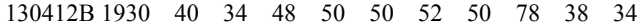

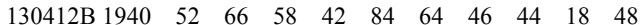
$\begin{array}{lllllllllll}130412 \mathrm{~B} 1950 & 70 & 28 & 42 & 58 & 48 & 52 & 68 & 54 & 70 & 32\end{array}$ $\begin{array}{lllllllllll}130412 \mathrm{~B} 1960 & 32 & 66 & 58 & 46 & 60 & 32 & 52 & 52 & 36 & 38\end{array}$

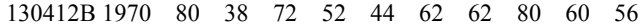

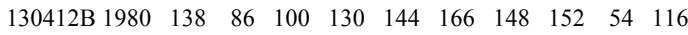

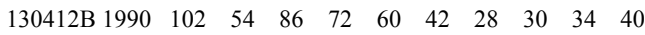
130412B $2000 \quad 82 \quad 78 \quad 38-9999$

130413A $1953 \quad 264 \quad 360 \quad 376 \quad 402 \quad 312 \quad 508 \quad 326$

$\begin{array}{llllllllllll}130413 \mathrm{~A} 1960 & 310 & 310 & 246 & 210 & 164 & 174 & 126 & 78 & 214 & 196\end{array}$ $\begin{array}{lllllllllll}130413 \mathrm{~A} 1970 & 112 & 100 & 108 & 144 & 158 & 134 & 118 & 96 & 146 & 106\end{array}$

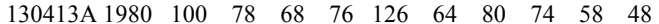
$\begin{array}{lllllllllll}130413 \mathrm{~A} 1990 & 92 & 206 & 110 & 132 & 92 & 62 & 96 & 70 & 76 & 70\end{array}$ 130413A $2000 \quad 100 \quad 74 \quad 66-9999$

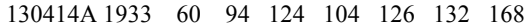

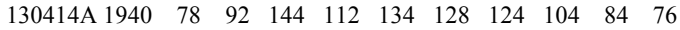

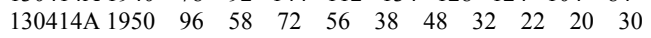
$\begin{array}{lllllllllll}130414 \mathrm{~A} 1960 & 30 & 98 & 104 & 156 & 142 & 92 & 146 & 110 & 168 & 154\end{array}$ 130414A 1970 $96 \begin{array}{llllllllll}96 & 130 & 100 & 84 & 60 & 84 & 132 & 66 & 28 & 92\end{array}$

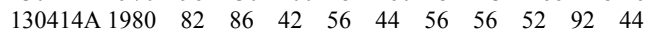
$\begin{array}{lllllllllll}130414 \mathrm{~A} 1990 & 40 & 68 & 50 & 52 & 36 & 26 & 34 & 24 & 22 & 26\end{array}$ 130414A $2000 \quad 42 \quad 48 \quad 36-9999$

130415A 1939316

$\begin{array}{lllllllllll}130415 \mathrm{~A} 1940 & 278 & 334 & 414 & 372 & 270 & 334 & 314 & 316 & 294 & 384\end{array}$ $\begin{array}{llllllllllll}130415 \text { A } 1950 & 294 & 258 & 256 & 264 & 236 & 278 & 328 & 374 & 312 & 252\end{array}$ $\begin{array}{lllllllllll}130415 \text { A } 1960 & 228 & 314 & 230 & 258 & 236 & 208 & 186 & 318 & 298 & 280\end{array}$ $\begin{array}{lllllllllll}130415 \text { A } 1970 & 276 & 290 & 262 & 278 & 328 & 350 & 260 & 266 & 446 & 268\end{array}$ $\begin{array}{lllllllllll}130415 \mathrm{~A} 1980 & 256 & 212 & 280 & 208 & 214 & 222 & 280 & 208 & 160 & 182\end{array}$ $\begin{array}{llllllllllll}130415 \text { A } 1990 & 184 & 140 & 192 & 166 & 138 & 152 & 116 & 160 & 152 & 106\end{array}$ 130415A $2000 \quad 194 \quad 148 \quad 136-9999$

$\begin{array}{lllllll}130415 \mathrm{~B} & 1935 & 122 & 264 & 434 & 392 & 310\end{array}$

$\begin{array}{lllllllllll}130415 \mathrm{~B} 1940 & 304 & 298 & 342 & 404 & 384 & 402 & 352 & 386 & 408 & 440\end{array}$ $\begin{array}{lllllllllll}130415 \mathrm{~B} 1950 & 320 & 284 & 286 & 324 & 276 & 308 & 300 & 346 & 326 & 258\end{array}$ $\begin{array}{lllllllllll}130415 \mathrm{~B} 1960 & 270 & 342 & 236 & 226 & 208 & 204 & 188 & 256 & 306 & 308\end{array}$ $\begin{array}{lllllllllll}130415 \mathrm{~B} 1970 & 318 & 288 & 296 & 246 & 284 & 332 & 266 & 278 & 366 & 250\end{array}$

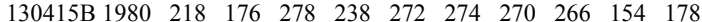
130415B $1990 \quad 194 \quad 160 \begin{array}{llllllllll}200 & 186 & 142 & 146 & 148 & 184 & 164 & 114\end{array}$ 130415B $2000 \quad 184 \quad 188 \quad 132-9999$

130416A $1938 \quad 312 \quad 322$

$\begin{array}{lllllllllll}130416 \text { A } 1940 & 302 & 264 & 352 & 368 & 288 & 336 & 312 & 328 & 372 & 412\end{array}$ $\begin{array}{lllllllllll}130416 \text { A } 1950 & 350 & 306 & 286 & 288 & 276 & 308 & 274 & 316 & 264 & 250\end{array}$ $\begin{array}{lllllllllll}130416 \text { A } 1960 & 278 & 276 & 216 & 188 & 212 & 214 & 198 & 244 & 258 & 248\end{array}$ $\begin{array}{lllllllllll}130416 \text { A } 1970 & 226 & 194 & 214 & 222 & 232 & 234 & 158 & 192 & 192 & 154\end{array}$ $\begin{array}{lllllllllll}130416 \text { A } 1980 & 206 & 184 & 186 & 132 & 112 & 122 & 138 & 108 & 70 & 102\end{array}$ $\begin{array}{lllllllllll}130416 \text { A } 1990 & 106 & 86 & 100 & 100 & 68 & 80 & 58 & 64 & 86 & 70\end{array}$ 130416A $2000 \quad 56 \quad 68 \quad 68-9999$

130416B $1936 \quad 102 \quad 252 \quad 282 \quad 264$

$\begin{array}{lllllllllll}130416 \mathrm{~B} 1940 & 310 & 276 & 312 & 280 & 288 & 296 & 254 & 296 & 286 & 310\end{array}$ $\begin{array}{lllllllllll}130416 \mathrm{~B} 1950 & 312 & 382 & 364 & 336 & 288 & 262 & 298 & 298 & 274 & 262\end{array}$ $\begin{array}{llllllllllll}130416 \mathrm{~B} 1960 & 248 & 228 & 258 & 250 & 158 & 160 & 164 & 150 & 178 & 166\end{array}$ $\begin{array}{lllllllllll}130416 \mathrm{~B} 1970 & 188 & 144 & 358 & 170 & 148 & 196 & 174 & 116 & 146 & 160\end{array}$ $\begin{array}{llllllllllll}130416 \mathrm{~B} & 1980 & 130 & 158 & 138 & 138 & 104 & 126 & 126 & 110 & 80 & 70\end{array}$

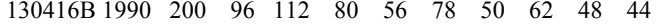
130416B $2000 \quad 24 \quad 50 \quad 32-9999$

130418A 1949116

130418A $1950 \quad 112 \quad 76 \quad 74 \quad 76 \quad 70 \quad 74 \quad 80 \quad 72 \quad 46 \quad 72$

$\begin{array}{lllllllllll}130418 \text { A } 1960 & 70 & 62 & 110 & 122 & 106 & 86 & 116 & 64 & 148 & 164\end{array}$

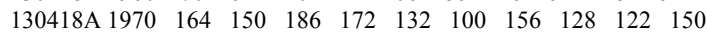

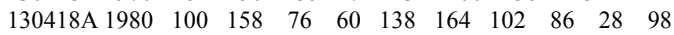

$\begin{array}{lllllllllll}130418 \mathrm{~A} 1990 & 92 & 24 & 122 & 90 & 84 & 58 & 74 & 66 & 68 & 60\end{array}$

130418A $2000 \quad 46 \quad 72 \quad 44-9999$

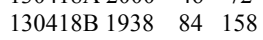

$\begin{array}{lllllllllll}130418 \mathrm{~B} 1940 & 154 & 120 & 118 & 100 & 110 & 162 & 118 & 124 & 154 & 130\end{array}$

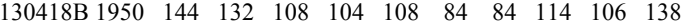
130418B 1960 $124 \begin{array}{llllllllll}100 & 54 & 68 & 102 & 152 & 216 & 216 & 132 & 98\end{array}$

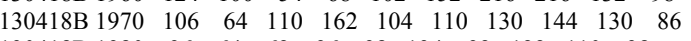

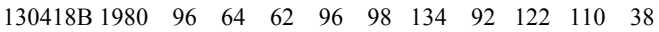

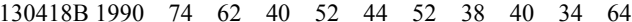
130418B $2000 \quad 20 \quad 34 \quad 46-9999$

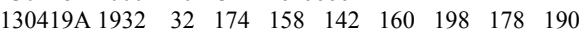

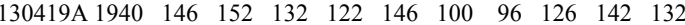
$\begin{array}{lllllllllll}130419 \text { A } 1950 & 104 & 92 & 70 & 130 & 96 & 112 & 102 & 100 & 184 & 136\end{array}$ $\begin{array}{lllllllllll}\text { 130419A } 1960 & 136 & 180 & 136 & 136 & 168 & 120 & 108 & 132 & 104 & 114\end{array}$ $\begin{array}{lllllllllll}130419 \text { A } 1970 & 132 & 140 & 130 & 140 & 176 & 188 & 118 & 144 & 178 & 124\end{array}$ $\begin{array}{lllllllllll}130419 \mathrm{~A} 1980 & 130 & 164 & 242 & 202 & 160 & 168 & 218 & 176 & 98 & 94\end{array}$ $\begin{array}{lllllllllll}130419 \text { A } 1990 & 130 & 102 & 132 & 128 & 120 & 106 & 114 & 126 & 124 & 128\end{array}$ 130419A 2000 $114 \quad 136 \quad 106-9999$

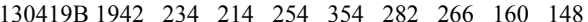

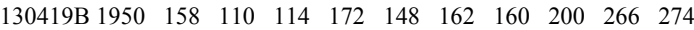

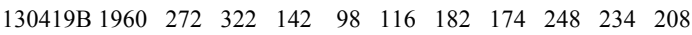

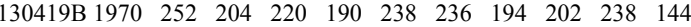

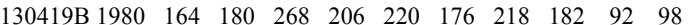

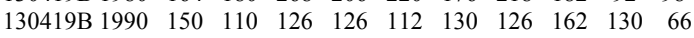
130419B $2000 \quad 94 \quad 122 \quad 102-9999$

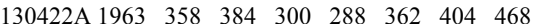

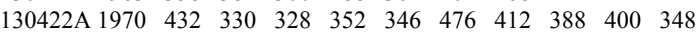
$\begin{array}{lllllllllll}130422 \mathrm{~A} 1980 & 402 & 382 & 472 & 422 & 472 & 414 & 410 & 304 & 192 & 244\end{array}$

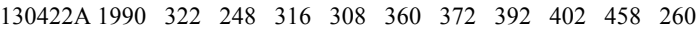
130422 A $2000 \quad 308 \quad 370 \quad 240-9999$

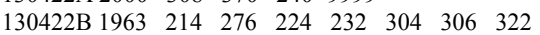

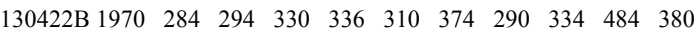
$\begin{array}{lllllllllll}130422 \mathrm{~B} 1980 & 410 & 484 & 552 & 462 & 348 & 346 & 336 & 302 & 168 & 320\end{array}$

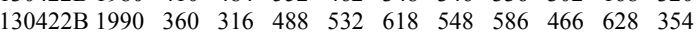
130422B $2000 \quad 506 \quad 470 \quad 232-9999$

130423B $1943 \quad 200 \quad 204 \quad 156 \quad 146 \quad 244 \quad 108 \quad 92$

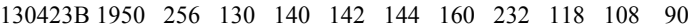

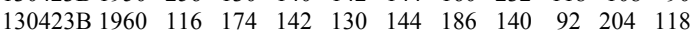

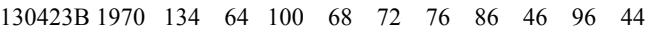

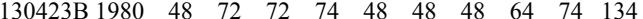

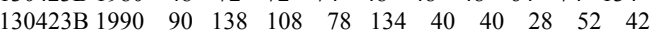
$130423 \mathrm{~B} 2000 \quad 58 \quad 56 \quad 38-9999$

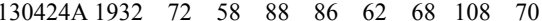

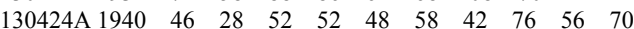
$\begin{array}{lllllllllll}130424 \mathrm{~A} 1950 & 74 & 82 & 70 & 60 & 78 & 62 & 16 & 40 & 42 & 52\end{array}$

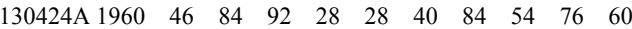

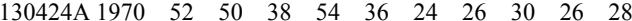
$\begin{array}{lllllllllll}130424 \mathrm{~A} 1980 & 14 & 22 & 8 & 26 & 38 & 30 & 34 & 28 & 28 & 14\end{array}$ $\begin{array}{llccccccccc}130424 \mathrm{~A} 1990 & 10 & 8 & 10 & 12 & 12 & 18 & 10 & 8 & 12 & 12\end{array}$ 130424A $2000 \quad 10 \quad 12 \quad 20-9999$

130424B $1932 \quad 122 \quad 48 \quad 106 \quad 138 \quad 92 \quad 54 \quad 42 \quad 64$

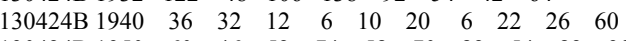
130424B 1950 $60 \begin{array}{llllllllll}46 & 52 & 74 & 58 & 70 & 82 & 54 & 82 & 90\end{array}$ 130424B 1960 $64 \begin{array}{llllllllll}86 & 56 & 84 & 90 & 38 & 60 & 54 & 58 & 38\end{array}$

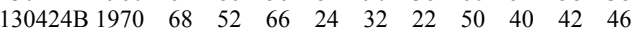

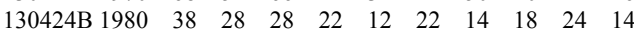
130424B 1990 $10 \begin{array}{llllllllll}10 & 14 & 16 & 18 & 20 & 30 & 58 & 42 & 18 & 6\end{array}$ 130424B $2000 \quad 8 \quad 8 \quad 34-9999$

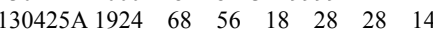
$\begin{array}{lllllllllll}130425 \mathrm{~A} 1930 & 14 & 36 & 22 & 18 & 28 & 34 & 32 & 20 & 68 & 48\end{array}$

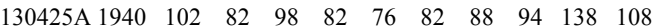

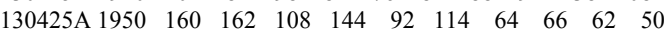

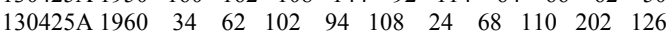

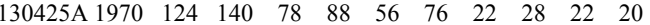
$\begin{array}{lllllllllll}130425 \mathrm{~A} 1980 & 40 & 28 & 112 & 146 & 50 & 44 & 46 & 42 & 12 & 30\end{array}$ $\begin{array}{lllllllllll}130425 \mathrm{~A} 1990 & 36 & 28 & 24 & 36 & 36 & 18 & 28 & 44 & 18 & 14\end{array}$ 130425 A $2000 \quad 16 \quad 10 \quad 12-9999$

$130425 \mathrm{~B} 1927 \quad 68 \quad 40 \quad 56$

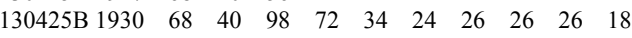
$\begin{array}{lllllllllll}130425 \mathrm{~B} 1940 & 38 & 30 & 20 & 22 & 26 & 26 & 46 & 40 & 36 & 82\end{array}$

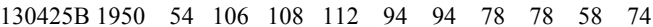

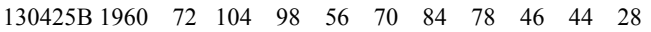

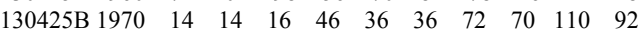

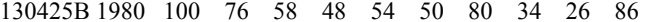
$\begin{array}{lllllllllll}130425 \mathrm{~B} 1990 & 96 & 92 & 44 & 42 & 90 & 128 & 82 & 154 & 78 & 112\end{array}$ 130425B $2000 \quad 90 \quad 76 \quad 52-9999$

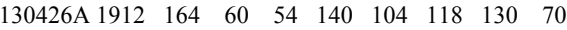

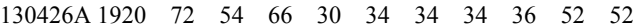

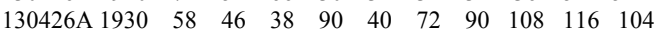
$\begin{array}{lllllllllll}130426 \text { A } 1940 & 122 & 106 & 104 & 182 & 132 & 152 & 140 & 124 & 162 & 142\end{array}$

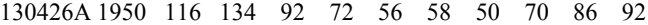
$\begin{array}{lllllllllll}130426 \mathrm{~A} 1960 & 34 & 44 & 60 & 24 & 28 & 22 & 26 & 32 & 16 & 34\end{array}$

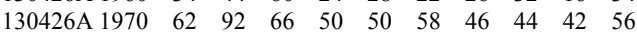




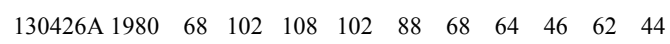

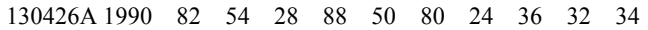
130426A $2000 \quad 30 \quad 54 \quad 36-9999$

130426B $1915 \quad 226 \quad 150 \quad 140 \quad 152 \quad 80$

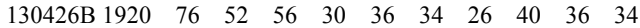

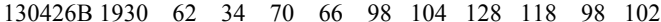

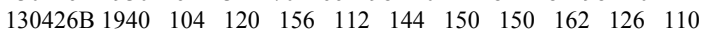
$\begin{array}{lllllllllll}130426 \mathrm{~B} 1950 & 124 & 102 & 66 & 46 & 52 & 88 & 116 & 92 & 78 & 48\end{array}$

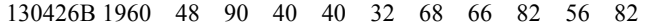

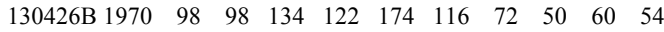

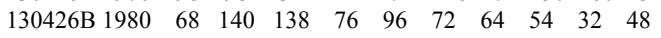

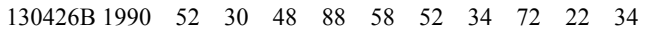
130426B 2000 $50 \quad 76 \quad 62-9999$

130427A $1925 \quad 48 \quad 60 \quad 44 \quad 50 \quad 42$

$\begin{array}{lllllllllll}130427 \mathrm{~A} 1930 & 54 & 78 & 52 & 124 & 100 & 166 & 114 & 176 & 158 & 82\end{array}$ $\begin{array}{lllllllllll}130427 \mathrm{~A} 1940 & 92 & 72 & 118 & 150 & 60 & 206 & 182 & 92 & 110 & 88\end{array}$ $\begin{array}{llllllllllll}130427 \mathrm{~A} 1950 & 126 & 106 & 106 & 98 & 84 & 84 & 74 & 98 & 118 & 82\end{array}$ $\begin{array}{lllllllllll}130427 \mathrm{~A} 1960 & 36 & 28 & 56 & 82 & 124 & 156 & 116 & 80 & 106 & 66\end{array}$ $\begin{array}{lllllllllll}130427 \mathrm{~A} 1970 & 96 & 154 & 120 & 128 & 142 & 134 & 128 & 154 & 154 & 226\end{array}$ $\begin{array}{lllllllllll}130427 \text { A } 1980 & 162 & 188 & 168 & 170 & 114 & 126 & 168 & 138 & 80 & 142\end{array}$

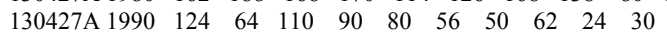
130427A $2000 \quad 20 \quad 16 \quad 62-9999$

$\begin{array}{lllllll}130427 \mathrm{~B} 1924 & 24 & 18 & 60 & 72 & 28 & 34\end{array}$

$\begin{array}{lllllllllll}130427 \mathrm{~B} 1930 & 38 & 66 & 84 & 56 & 130 & 130 & 158 & 128 & 176 & 142\end{array}$

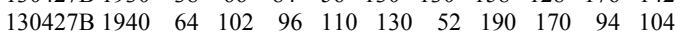
$\begin{array}{lllllllllll}130427 \mathrm{~B} 1950 & 78 & 140 & 152 & 128 & 94 & 80 & 88 & 76 & 102 & 132\end{array}$ $\begin{array}{lllllllllll}130427 \mathrm{~B} 1960 & 84 & 34 & 26 & 84 & 92 & 122 & 170 & 110 & 76 & 110\end{array}$ $\begin{array}{lllllllllll}130427 \mathrm{~B} 1970 & 56 & 100 & 168 & 132 & 138 & 138 & 144 & 126 & 190 & 182\end{array}$ $\begin{array}{lllllllllll}130427 \mathrm{~B} 1980 & 122 & 118 & 172 & 198 & 170 & 106 & 100 & 164 & 222 & 140\end{array}$ 130427B 1990 $70 \begin{array}{llllllllll}70 & 140 & 120 & 58 & 122 & 98 & 90 & 54 & 48 & 68\end{array}$ 130427B $2000 \quad 28 \quad 32 \quad 102-9999$

130501A $1928 \quad 48 \quad 80$

$\begin{array}{lllllllllll}130501 \mathrm{~A} 1930 & 82 & 92 & 136 & 138 & 156 & 116 & 128 & 148 & 120 & 68\end{array}$ $\begin{array}{lllllllllll}130501 \mathrm{~A} 1940 & 52 & 46 & 50 & 70 & 50 & 24 & 42 & 8 & 32 & 34\end{array}$

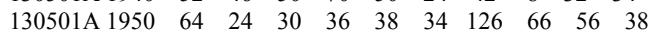

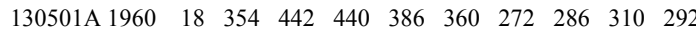

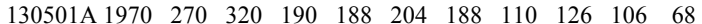

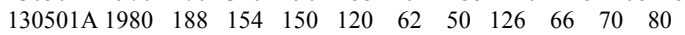
$\begin{array}{lllllllllll}130501 \mathrm{~A} 1990 & 66 & 82 & 232 & 140 & 98 & 74 & 84 & 122 & 92 & 92\end{array}$ 130501A $2000 \quad 86 \quad 64 \quad 58-9999$

130501B $1938 \quad 116 \quad 60$

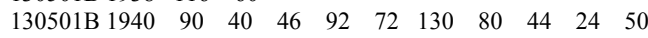

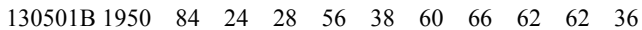

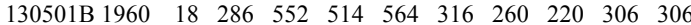

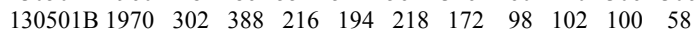

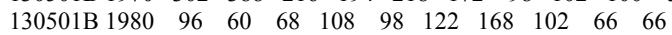
$\begin{array}{lllllllllll}130501 \mathrm{~B} 1990 & 40 & 62 & 192 & 154 & 80 & 88 & 92 & 126 & 100 & 68\end{array}$ 130501B $2000 \quad 58 \quad 62 \quad 38-9999$

$\begin{array}{lllllllllll}130502 \text { A } 1930 & 132 & 218 & 314 & 280 & 386 & 290 & 232 & 248 & 220 & 162\end{array}$ $\begin{array}{lllllllllll}130502 \mathrm{~A} 1940 & 232 & 126 & 80 & 96 & 194 & 102 & 76 & 72 & 70 & 68\end{array}$

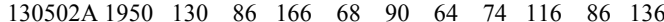
$\begin{array}{lllllllllll}130502 \mathrm{~A} 1960 & 252 & 274 & 328 & 384 & 552 & 398 & 440 & 344 & 378 & 424\end{array}$ $\begin{array}{llllllllllll}130502 \mathrm{~A} 1970 & 398 & 286 & 394 & 446 & 532 & 468 & 322 & 364 & 380 & 436\end{array}$ $\begin{array}{lllllllllll}130502 \mathrm{~A} 1980 & 294 & 132 & 256 & 264 & 228 & 454 & 448 & 318 & 148 & 244\end{array}$ $\begin{array}{lllllllllll}130502 \mathrm{~A} 1990 & 148 & 542 & 312 & 438 & 286 & 310 & 400 & 316 & 292 & 356\end{array}$ 130502A $2000 \quad 194 \quad 236 \quad 442-9999$

$\begin{array}{llllllll}130502 B & 1944 & 228 & 224 & 228 & 176 & 102 & 76\end{array}$

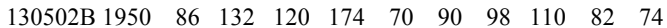

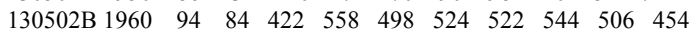

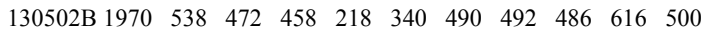

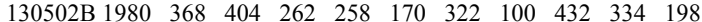

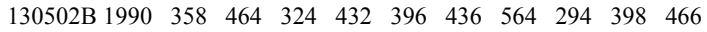
130502B $2000 \quad 196 \quad 192 \quad 284-9999$

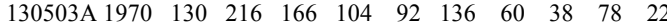

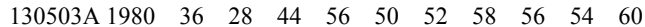
$\begin{array}{lllllllllll}130503 \mathrm{~A} 1990 & 30 & 32 & 16 & 86 & 54 & 28 & 32 & 26 & 36 & 54\end{array}$ 130503A $2000 \quad 30 \quad 22 \quad 32-9999$

$130504 \mathrm{~A} 1948 \quad 52 \quad 32$

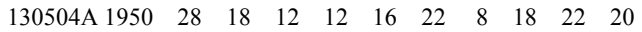
$\begin{array}{lllllllllll}130504 \mathrm{~A} 1960 & 22 & 30 & 20 & 20 & 32 & 134 & 172 & 136 & 160 & 144\end{array}$

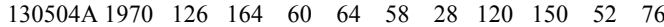

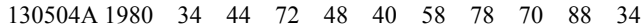
$\begin{array}{lllllllllll}130504 \mathrm{~A} 1990 & 38 & 58 & 84 & 34 & 94 & 14 & 16 & 26 & 38 & 58\end{array}$ 130504A $2000 \quad 24 \quad 38 \quad 66-9999$

130504B $\begin{array}{lllll}1966 & 122 & 178 & 182 & 172\end{array}$

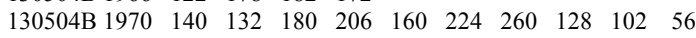

130504B 1980 $66 \begin{array}{llllllllll}176 & 84 & 52 & 18 & 16 & 86 & 54 & 80 & 80\end{array}$

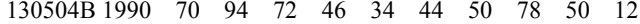

130504B 2000 $76 \quad 64 \quad 54-9999$

$\begin{array}{lll}130505 \text { A } 1948 & 124 & 32\end{array}$
130505A $1950 \quad 64 \quad 56 \quad 18 \quad 32 \quad 24 \quad 20 \quad 40 \quad 24 \quad 24 \quad 22$ $\begin{array}{lllllllllll}130505 \text { A } 1960 & 44 & 242 & 266 & 224 & 236 & 234 & 236 & 274 & 286 & 232\end{array}$

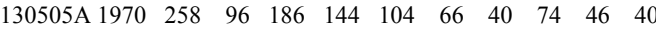
$\begin{array}{lllllllllll}130505 \mathrm{~A} 1980 & 30 & 28 & 34 & 128 & 58 & 46 & 84 & 46 & 48 & 50\end{array}$ $\begin{array}{lllllllllll}130505 \mathrm{~A} 1990 & 60 & 48 & 40 & 58 & 52 & 144 & 56 & 64 & 32 & 28\end{array}$ 130505 A $2000 \quad 50 \quad 22 \quad 36-9999$

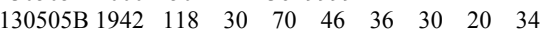

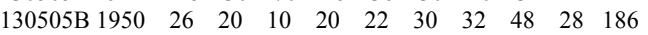

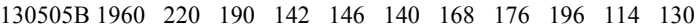

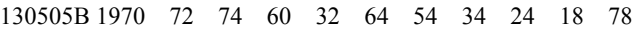
$\begin{array}{lllllllllll}130505 \mathrm{~B} 1980 & 70 & 38 & 28 & 30 & 44 & 40 & 50 & 46 & 48 & 42\end{array}$

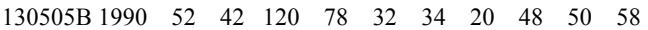
130505 B $2000 \quad 72 \quad 90 \quad 86-9999$

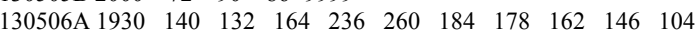

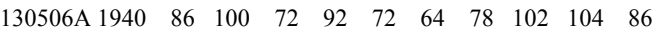
130506A 1950 $74 \begin{array}{llllllllll}738 & 98 & 112 & 78 & 98 & 96 & 62 & 156 & 44\end{array}$

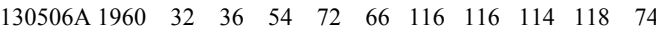

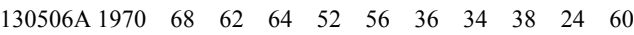

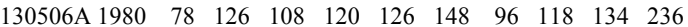
$\begin{array}{lllllllllll}130506 \text { A } 1990 & 184 & 236 & 264 & 238 & 168 & 166 & 186 & 152 & 140 & 120\end{array}$ 130506A $2000 \quad 48 \quad 140 \quad 90-9999$

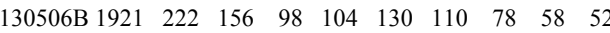

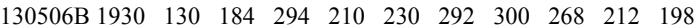

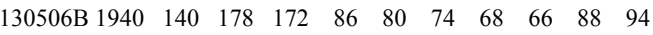

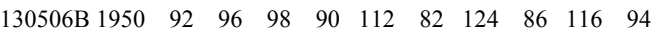

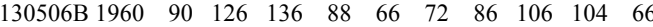

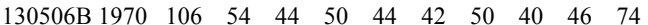

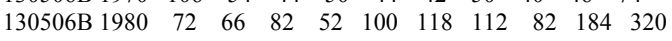

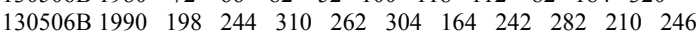
130506B $2000 \quad 156 \quad 124 \quad 122-9999$

130507A $1937 \quad 32 \quad 126 \quad 134$

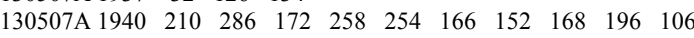
$\begin{array}{lllllllllll}130507 \text { A } 1950 & 106 & 142 & 144 & 186 & 112 & 84 & 154 & 90 & 76 & 92\end{array}$

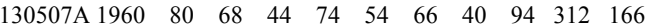

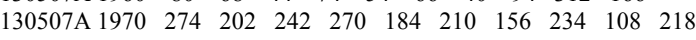

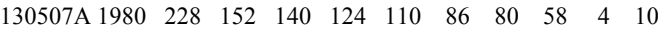
$\begin{array}{lllllllllll}130507 \mathrm{~A} 1990 & 22 & 14 & 14 & 20 & 16 & 30 & 34 & 36 & 44 & 48\end{array}$ 130507A $2000 \quad 26 \quad 46 \quad 58-9999$

130507B $1934 \quad 174 \quad 144 \quad 126 \quad 124 \quad 72 \quad 74$

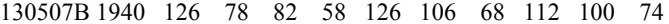
130507B 1950 $66 \begin{array}{llllllllll}80 & 86 & 78 & 62 & 34 & 42 & 56 & 44 & 76\end{array}$ $\begin{array}{lllllllllll}130507 \mathrm{~B} 1960 & 52 & 36 & 34 & 40 & 260 & 340 & 194 & 316 & 222 & 202\end{array}$ $\begin{array}{lllllllllll}130507 \mathrm{~B} 1970 & 286 & 270 & 224 & 108 & 104 & 108 & 104 & 200 & 92 & 132\end{array}$

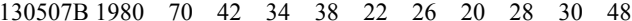

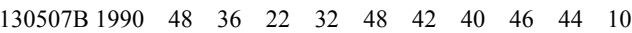
130507B $2000 \quad 12 \quad 12 \quad 22-9999$

130508A $1938266 \quad 236$

$\begin{array}{lllllllllll}130508 \text { A } 1940 & 204 & 194 & 248 & 124 & 166 & 148 & 158 & 162 & 152 & 134\end{array}$

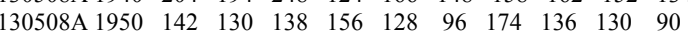

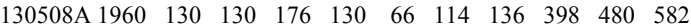

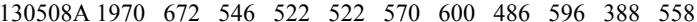
$\begin{array}{lllllllllll}130508 \text { A } 1980 & 448 & 314 & 408 & 416 & 262 & 260 & 144 & 308 & 218 & 194\end{array}$ $\begin{array}{llllllllllll}130508 \text { A } 1990 & 262 & 234 & 446 & 374 & 218 & 90 & 98 & 202 & 112 & 314\end{array}$ 130508A 2000 $198 \quad 176 \quad 156-9999$

130508B $1946 \quad 294 \quad 262 \quad 104 \quad 278$

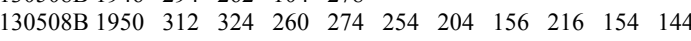

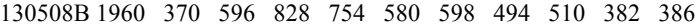

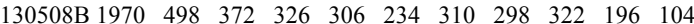

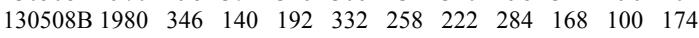
$\begin{array}{lllllllllll}130508 \mathrm{~B} 1990 & 254 & 54 & 152 & 176 & 72 & 84 & 60 & 66 & 90 & 108\end{array}$ 130508B $2000 \quad 68 \quad 42 \quad 46-9999$

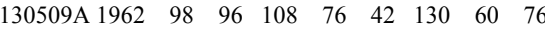

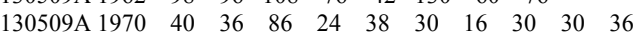
$\begin{array}{lllllllllll}130509 \text { A } 1980 & 30 & 66 & 234 & 224 & 188 & 140 & 104 & 124 & 102 & 132\end{array}$

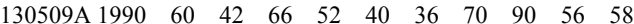
130509A $2000 \quad 50 \quad 52 \quad 46-9999$ $\begin{array}{lllllll}130511 \mathrm{~A} 1934 & 70 & 124 & 94 & 96 & 144 & 108\end{array}$

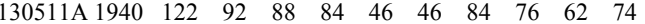
$\begin{array}{lllllllllll}130511 \mathrm{~A} 1950 & 66 & 86 & 78 & 38 & 70 & 604 & 408 & 272 & 92 & 132\end{array}$ $\begin{array}{lllllllllll}130511 \mathrm{~A} 1960 & 208 & 140 & 50 & 158 & 226 & 74 & 68 & 118 & 82 & 284\end{array}$

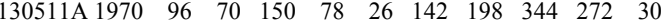

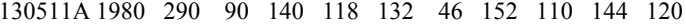
$\begin{array}{lllllllllll}130511 \mathrm{~A} 1990 & 120 & 212 & 98 & 164 & 202 & 92 & 82 & 154 & 130 & 180\end{array}$ 130511A $2000 \quad 116 \quad 140 \quad 130-9999$

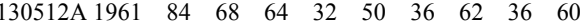

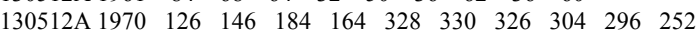
$\begin{array}{lllllllllll}130512 \text { A } 1980 & 334 & 302 & 186 & 224 & 208 & 80 & 106 & 296 & 156 & 146\end{array}$ $\begin{array}{lllllllllll}130512 \mathrm{~A} 1990 & 52 & 54 & 28 & 30 & 34 & 28 & 20 & 42 & 122 & 158\end{array}$ 130512A $2000 \quad 132 \quad 154 \quad 230-9999$ 130512B 195932 


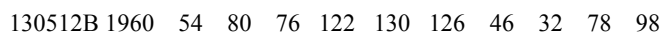

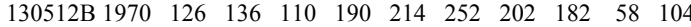

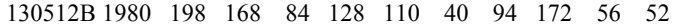
$\begin{array}{lllllllllll}130512 \mathrm{~B} 1990 & 46 & 42 & 32 & 26 & 28 & 22 & 22 & 24 & 56 & 58\end{array}$ 130512B $2000 \quad 82 \quad 128 \quad 108-9999$

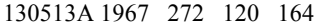

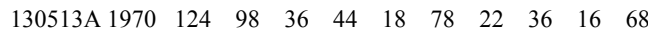
$\begin{array}{lllllllllll}130513 \mathrm{~A} 1980 & 38 & 38 & 72 & 84 & 32 & 28 & 26 & 28 & 22 & 18\end{array}$ $\begin{array}{lllllllllll}130513 \mathrm{~A} 1990 & 60 & 30 & 30 & 54 & 32 & 42 & 68 & 30 & 38 & 56\end{array}$ 130513A $2000 \quad 48 \quad 58 \quad 42-9999$

$\begin{array}{llllllll}130515 \text { A } 1963 & 32 & 26 & 50 & 50 & 54 & 76 & 90\end{array}$

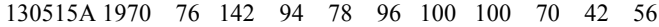
$\begin{array}{lllllllllll}130515 \mathrm{~A} 1980 & 92 & 70 & 24 & 16 & 48 & 224 & 134 & 126 & 112 & 34\end{array}$ $\begin{array}{lllllllllll}130515 \mathrm{~A} 1990 & 38 & 50 & 66 & 10 & 30 & 106 & 72 & 80 & 84 & 112\end{array}$ 130515A $2000 \quad 36 \quad 42 \quad 30-9999$

$130515 \mathrm{~B} 1956 \quad 66 \quad 36 \quad 32 \quad 30$

$\begin{array}{lllllllllll}130515 \mathrm{~B} 1960 & 22 & 12 & 28 & 14 & 52 & 42 & 36 & 28 & 64 & 258\end{array}$

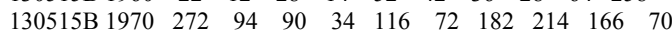

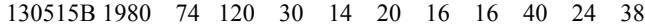

$\begin{array}{lllllllllll}130515 \mathrm{~B} 1990 & 38 & 22 & 10 & 36 & 24 & 36 & 60 & 74 & 76 & 90\end{array}$

130515B $2000 \quad 80 \quad 26 \quad 74-9999$

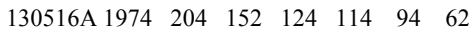

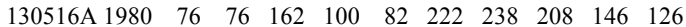
$\begin{array}{lllllllllll}130516 \mathrm{~A} 1990 & 38 & 44 & 94 & 134 & 110 & 134 & 110 & 108 & 102 & 106\end{array}$ 130516A $2000 \quad 60 \quad 124 \quad 30-9999$

130517A $1984 \quad 276 \quad 236 \quad 236 \quad 200 \quad 230 \quad 30$

$\begin{array}{lllllllllll}130517 \mathrm{~A} 1990 & 146 & 200 & 66 & 84 & 116 & 74 & 126 & 76 & 76 & 70\end{array}$

130517A $2000 \quad 110 \quad 106 \quad 36-9999$

$\begin{array}{lllllllll}130518 \text { A } 1972 & 184 & 248 & 240 & 314 & 234 & 174 & 90 & 62\end{array}$

$\begin{array}{lllllllllll}130518 \text { A } 1980 & 186 & 144 & 244 & 242 & 174 & 138 & 82 & 74 & 30 & 64\end{array}$

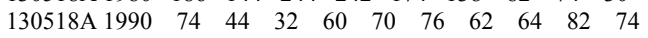

130518A $2000 \quad 80 \quad 102 \quad 88-9999$

130518B $1967 \quad 322 \quad 238 \quad 242$

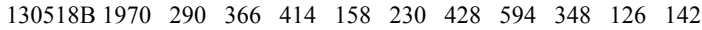

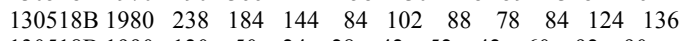

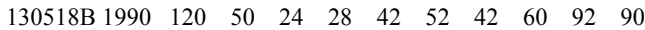

130518B $2000 \quad 88 \quad 20 \quad 36-9999$

$\begin{array}{llllll}130601 \text { A } 1945 & 338 & 204 & 204 & 228 & 224\end{array}$

$\begin{array}{lllllllllll}130601 \mathrm{~A} 1950 & 222 & 220 & 208 & 398 & 194 & 144 & 146 & 108 & 106 & 76\end{array}$

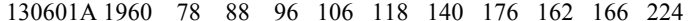

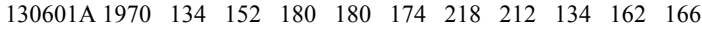

$\begin{array}{llllllllllll}130601 \mathrm{~A} & 1980 & 188 & 186 & 182 & 264 & 204 & 194 & 142 & 174 & 132 & 96\end{array}$

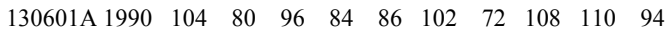

130601A $2000 \quad 82 \quad 90 \quad 96-9999$

130601B $1947 \quad 136 \quad 232 \quad 178$

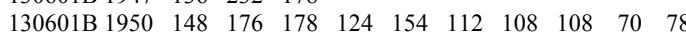

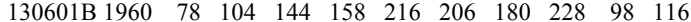

$\begin{array}{lllllllllll}130601 \mathrm{~B} 1970 & 118 & 142 & 154 & 166 & 154 & 112 & 106 & 166 & 162 & 158\end{array}$

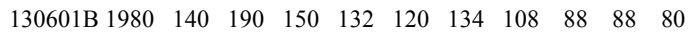

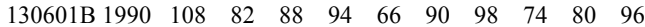

130601B $2000 \quad 144 \quad 100 \quad 82-9999$

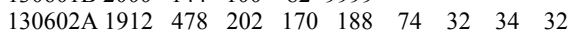

$\begin{array}{lllllllllll}130602 \mathrm{~A} 1920 & 26 & 28 & 42 & 64 & 72 & 74 & 132 & 188 & 286 & 394\end{array}$

$\begin{array}{lllllllllll}130602 \text { A } 1930 & 382 & 366 & 370 & 304 & 252 & 232 & 230 & 194 & 178 & 198\end{array}$

$\begin{array}{lllllllllll}130602 \mathrm{~A} 1940 & 314 & 120 & 146 & 136 & 114 & 104 & 76 & 62 & 74 & 176\end{array}$

$\begin{array}{lllllllllll}130602 \text { A } 1950 & 118 & 96 & 136 & 158 & 162 & 192 & 192 & 198 & 144 & 136\end{array}$

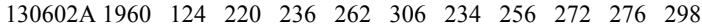

$\begin{array}{lllllllllll}130602 \mathrm{~A} 1970 & 272 & 204 & 184 & 220 & 270 & 232 & 182 & 198 & 190 & 164\end{array}$

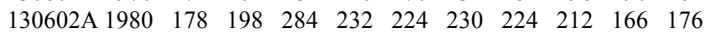

$\begin{array}{llllllllllll}130602 \mathrm{~A} 1990 & 220 & 162 & 182 & 176 & 166 & 180 & 210 & 202 & 216 & 158\end{array}$

130602 A $2000 \quad 180 \quad 216 \quad 146-9999$

130602B $1924 \quad 72 \quad 104 \quad 150 \quad 208 \quad 216 \quad 392$

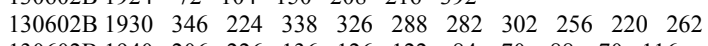

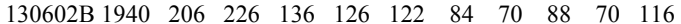

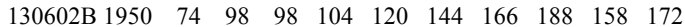

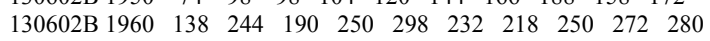

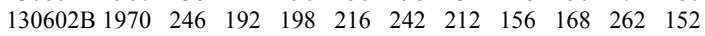

$\begin{array}{llllllllllll}130602 \mathrm{~B} 1980 & 240 & 190 & 204 & 158 & 186 & 240 & 242 & 184 & 132 & 144\end{array}$

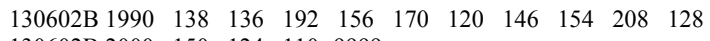

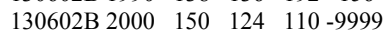

130603A 1969144

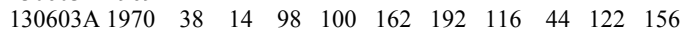

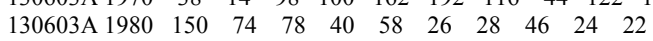

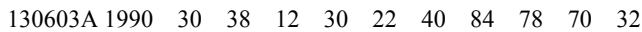

130603A $2000 \quad 64 \quad 50 \quad 56-9999$

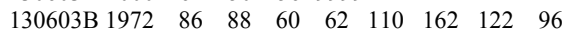

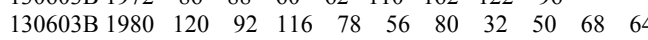

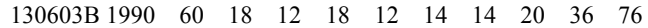

130603B $2000 \quad 40 \quad 54 \quad 72-9999$

130604A $1947 \quad 150 \quad 156 \quad 146$

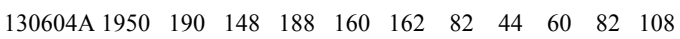

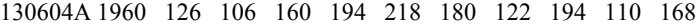
$\begin{array}{lllllllllll}130604 \mathrm{~A} 1970 & 232 & 188 & 184 & 180 & 256 & 172 & 240 & 162 & 306 & 256\end{array}$ $\begin{array}{lllllllllll}130604 \mathrm{~A} 1980 & 220 & 176 & 160 & 112 & 124 & 148 & 116 & 118 & 140 & 126\end{array}$

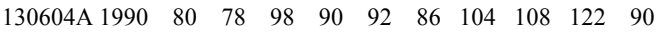
130604A $2000 \quad 96 \quad 98 \quad 134-9999$

$\begin{array}{llllllll}130605 \text { A } 1943 & 220 & 176 & 244 & 134 & 182 & 102 & 152\end{array}$

$\begin{array}{lllllllllll}130605 \text { A } 1950 & 80 & 80 & 128 & 154 & 132 & 166 & 202 & 212 & 220 & 228\end{array}$

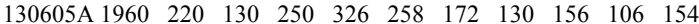

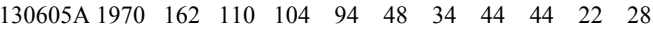

$\begin{array}{lllllllllll}130605 \text { A } 1980 & 76 & 150 & 200 & 222 & 234 & 208 & 198 & 106 & 66 & 124\end{array}$

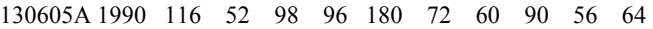
130605A $2000 \quad 148 \quad 200 \quad 118-9999$

130605B 1939346

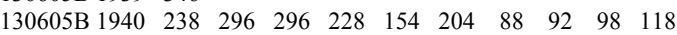

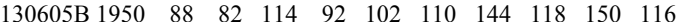

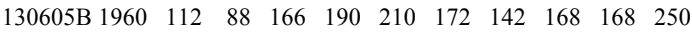

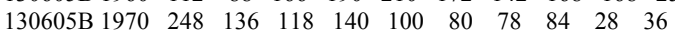

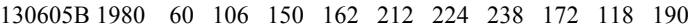

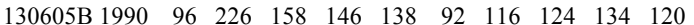
130605B $2000 \quad 150 \quad 176 \quad 100-9999$

130606A $1945 \quad 176 \quad 180 \quad 150 \quad 544 \quad 338$

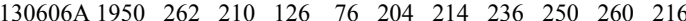
$\begin{array}{lllllllllll}130606 \text { A } 1960 & 228 & 126 & 248 & 194 & 232 & 496 & 172 & 222 & 162 & 262\end{array}$ $\begin{array}{lllllllllll}130606 \text { A } 1970 & 200 & 224 & 246 & 202 & 240 & 146 & 202 & 164 & 112 & 190\end{array}$

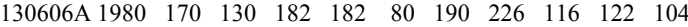

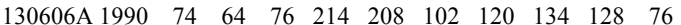
130606A $2000 \quad 104 \quad 108 \quad 116-9999$

130606B $1948 \quad 118 \quad 296$

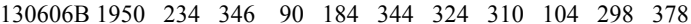

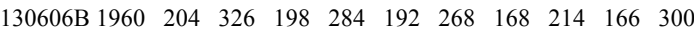

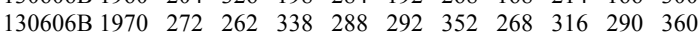

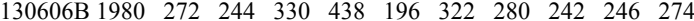

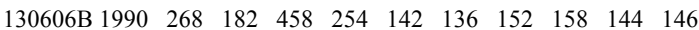
130606B $2000 \quad 74 \quad 54 \quad 54-9999$

130607A $1945 \quad 60 \quad 48 \quad 72 \quad 36 \quad 12$

$\begin{array}{lllllllllll}130607 \mathrm{~A} 1950 & 58 & 112 & 206 & 240 & 156 & 208 & 188 & 176 & 190 & 148\end{array}$

$\begin{array}{lllllllllll}130607 \mathrm{~A} 1960 & 88 & 86 & 68 & 80 & 36 & 84 & 110 & 48 & 90 & 68\end{array}$

$\begin{array}{lllllllllll}130607 \mathrm{~A} 1970 & 44 & 74 & 42 & 14 & 42 & 26 & 34 & 38 & 52 & 40\end{array}$

130607A 1980 $60 \begin{array}{llllllllll}60 & 46 & 126 & 58 & 48 & 54 & 88 & 64 & 72\end{array}$

$\begin{array}{lllllllllll}130607 \mathrm{~A} 1990 & 26 & 48 & 112 & 26 & 34 & 30 & 58 & 40 & 36 & 42\end{array}$

130607A $2000 \quad 26 \quad 22 \quad 46-9999$

130608 A $1936 \quad 62 \quad 1004 \quad 520 \quad 560$

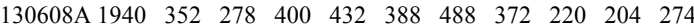

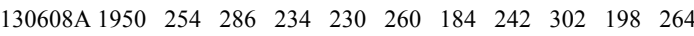
$\begin{array}{lllllllllll}130608 \text { A } 1960 & 248 & 154 & 126 & 132 & 172 & 178 & 108 & 112 & 128 & 58\end{array}$ $\begin{array}{llllllllllll}130608 \text { A } 1970 & 68 & 118 & 162 & 168 & 148 & 124 & 106 & 184 & 168 & 98\end{array}$ $\begin{array}{llllllllllll}130608 \text { A } 1980 & 102 & 102 & 136 & 164 & 98 & 146 & 128 & 106 & 56 & 98\end{array}$

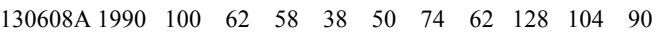
130608A $2000 \quad 116 \quad 76 \quad 168-9999$

130608B $1945 \quad 302 \quad 324 \quad 356 \quad 254 \quad 172$

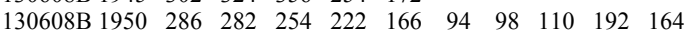
$\begin{array}{llllllllllll}130608 \mathrm{~B} & 1960 & 146 & 194 & 136 & 150 & 176 & 192 & 170 & 186 & 142 & 124\end{array}$

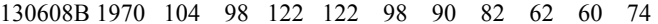

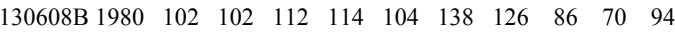

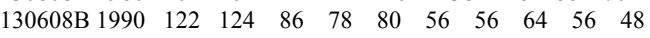
130608B $2000 \quad 52 \quad 52 \quad 54-9999$

130609A $1938 \quad 170 \quad 272$

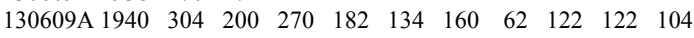
$\begin{array}{lllllllllll}130609 \text { A } 1950 & 114 & 186 & 290 & 202 & 226 & 278 & 296 & 212 & 392 & 332\end{array}$ $\begin{array}{lllllllllll}130609 \text { A } 1960 & 326 & 412 & 404 & 308 & 416 & 532 & 390 & 300 & 318 & 364\end{array}$ $\begin{array}{lllllllllll}\text { 130609A } 1970 & 240 & 246 & 294 & 230 & 238 & 182 & 106 & 82 & 144 & 156\end{array}$ $\begin{array}{lllllllllll}130609 \text { A } 1980 & 166 & 106 & 112 & 212 & 172 & 132 & 110 & 170 & 62 & 42\end{array}$ $\begin{array}{lllllllllll}130609 \text { A } 1990 & 564 & 122 & 100 & 76 & 104 & 186 & 162 & 164 & 182 & 164\end{array}$ 130609A $2000 \quad 178 \quad 202 \quad 230-9999$

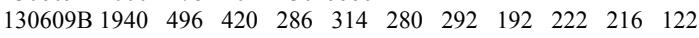

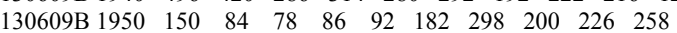

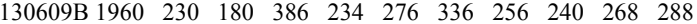

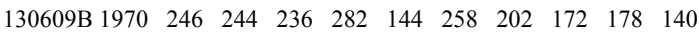

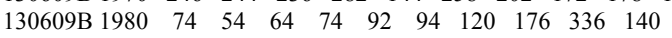

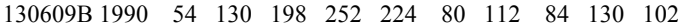
130609B 2000 $120 \quad 106 \quad 122-9999$

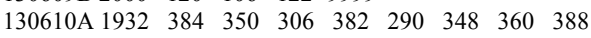

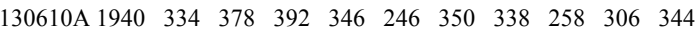
130610A 1950 $288 \begin{array}{llllllllll}270 & 246 & 288 & 258 & 254 & 230 & 290 & 336 & 272\end{array}$ $\begin{array}{lllllllllll}130610 \text { A } 1960 & 358 & 456 & 342 & 406 & 408 & 328 & 342 & 398 & 390 & 286\end{array}$ $\begin{array}{lllllllllll}130610 \text { A } 1970 & 352 & 242 & 260 & 248 & 216 & 238 & 254 & 234 & 362 & 224\end{array}$ $\begin{array}{lllllllllll}130610 \text { A } 1980 & 288 & 270 & 328 & 276 & 274 & 328 & 292 & 284 & 216 & 302\end{array}$

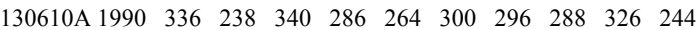
130610A $2000 \quad 202 \quad 348 \quad 254-9999$ 
130610B $1922 \quad 262 \quad 204 \quad 208214 \quad 190 \quad 166 \quad 174 \quad 230$

$\begin{array}{lllllllllll}130610 \mathrm{~B} 1930 & 214 & 284 & 354 & 348 & 370 & 396 & 266 & 292 & 286 & 268\end{array}$ $\begin{array}{lllllllllll}130610 \mathrm{~B} 1940 & 294 & 354 & 428 & 388 & 282 & 364 & 346 & 296 & 348 & 370\end{array}$ 130610B 1950 $310 \begin{array}{llllllllll}328 & 254 & 362 & 360 & 384 & 308 & 350 & 400 & 312\end{array}$

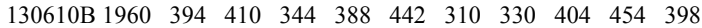
$\begin{array}{lllllllllll}130610 \mathrm{~B} 1970 & 404 & 342 & 450 & 472 & 486 & 506 & 416 & 328 & 428 & 356\end{array}$

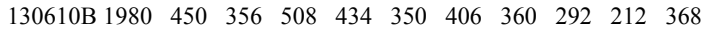
130610B 1990 $562 \begin{array}{lllllllll}366 & 382 & 332 & 286 & 310 & 354 & 426 & 506 & 282\end{array}$ 130610B $2000 \quad 286 \quad 340 \quad 236-9999$

130611A $1938 \quad 692 \quad 634$

$\begin{array}{lllllllllll}\text { 130611A } 1940 & 688 & 680 & 692 & 700 & 606 & 702 & 590 & 704 & 690 & 760\end{array}$

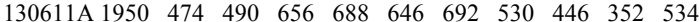

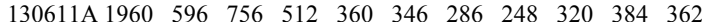
$\begin{array}{llllllllllll}130611 \mathrm{~A} 1970 & 468 & 368 & 336 & 296 & 370 & 334 & 244 & 268 & 396 & 252\end{array}$

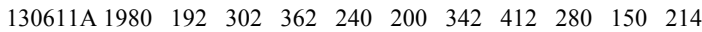

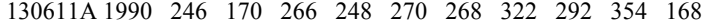
130611A $2000 \quad 440 \quad 212 \quad 148-9999$

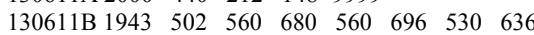

130611B 1950 $48 \begin{array}{llllllllll}416 & 506 & 594 & 526 & 568 & 556 & 502 & 420 & 556\end{array}$

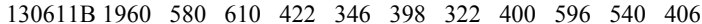
$\begin{array}{lllllllllll}130611 \mathrm{~B} 1970 & 472 & 462 & 592 & 634 & 842 & 916 & 548 & 632 & 658 & 444\end{array}$

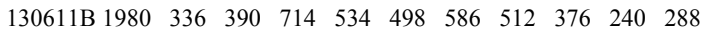

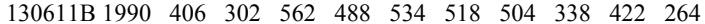
130611B $2000 \quad 514 \quad 314 \quad 142-9999$

130612A $1917 \quad 96 \quad 68 \quad 122$

$\begin{array}{lllllllllll}130612 \mathrm{~A} 1920 & 54 & 50 & 40 & 30 & 20 & 32 & 54 & 98 & 178 & 278\end{array}$

$\begin{array}{lllllllllll}130612 \text { A } 1930 & 226 & 302 & 208 & 290 & 282 & 306 & 244 & 258 & 346 & 372\end{array}$ $\begin{array}{llllllllllll}130612 \text { A } 1940 & 368 & 268 & 450 & 354 & 302 & 382 & 254 & 274 & 234 & 242\end{array}$ $\begin{array}{lllllllllll}130612 \text { A } 1950 & 148 & 156 & 134 & 128 & 64 & 152 & 62 & 112 & 108 & 52\end{array}$

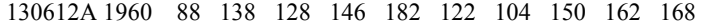

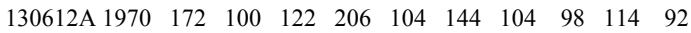

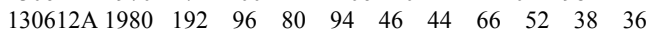
$\begin{array}{lllllllllll}130612 \mathrm{~A} 1990 & 56 & 46 & 82 & 144 & 142 & 70 & 74 & 80 & 104 & 116\end{array}$ 130612A $2000 \quad 146 \quad 158 \quad 124-9999$

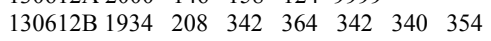

$\begin{array}{lllllllllll}130612 \mathrm{~B} 1940 & 210 & 322 & 458 & 364 & 206 & 516 & 494 & 296 & 304 & 194\end{array}$

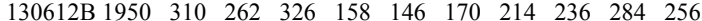

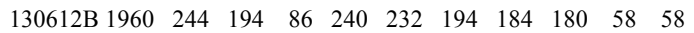

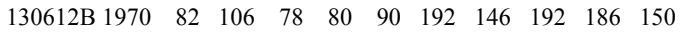

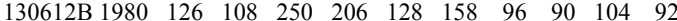
$\begin{array}{lllllllllll}130612 \mathrm{~B} 1990 & 76 & 42 & 212 & 126 & 134 & 68 & 76 & 112 & 128 & 114\end{array}$ 130612B $2000 \quad 128 \quad 134 \quad 108-9999$

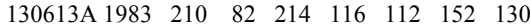

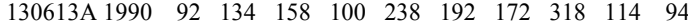
130613A $2000 \quad 46 \quad 42 \quad 64-9999$

130613B $1978 \quad 92 \quad 106$

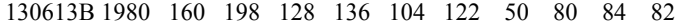

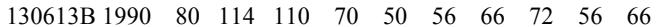

130613B 2000 $70 \quad 50 \quad 56-9999$

130614A $1933 \quad 90 \quad 282 \quad 366 \quad 340 \quad 372 \quad 346 \quad 340$

130614A 1940 $340 \begin{array}{llllllllll}254 & 270 & 282 & 238 & 294 & 208 & 294 & 258 & 264\end{array}$ $\begin{array}{lllllllllll}130614 \text { A } 1950 & 182 & 176 & 174 & 222 & 208 & 262 & 226 & 244 & 246 & 236\end{array}$

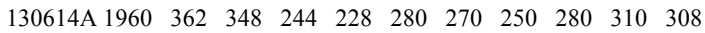
$\begin{array}{llllllllllll}130614 \mathrm{~A} 1970 & 242 & 222 & 206 & 252 & 296 & 318 & 206 & 162 & 266 & 208\end{array}$ $\begin{array}{lllllllllll}130614 \text { A } 1980 & 222 & 206 & 296 & 268 & 244 & 304 & 306 & 302 & 180 & 252\end{array}$ $\begin{array}{lllllllllll}130614 \mathrm{~A} 1990 & 260 & 198 & 262 & 210 & 248 & 168 & 226 & 184 & 200 & 144\end{array}$ 130614A $2000 \quad 224 \quad 234 \quad 156-9999$

130614B 1949194

$\begin{array}{lllllllllll}130614 \mathrm{~B} 1950 & 396 & 228 & 230 & 216 & 278 & 248 & 272 & 182 & 264 & 184\end{array}$

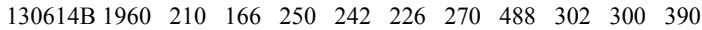
$\begin{array}{lllllllllll}130614 \mathrm{~B} 1970 & 360 & 342 & 294 & 350 & 374 & 466 & 348 & 310 & 354 & 254\end{array}$ 130614B 1980 $258 \begin{array}{llllllllll}230 & 288 & 280 & 250 & 346 & 364 & 258 & 186 & 210\end{array}$ $\begin{array}{lllllllllll}130614 \mathrm{~B} 1990 & 228 & 180 & 242 & 216 & 200 & 202 & 292 & 294 & 382 & 210\end{array}$ 130614B $2000 \quad 252 \quad 284 \quad 184-9999$

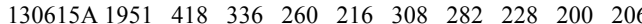
$\begin{array}{lllllllllll}130615 \mathrm{~A} 1960 & 216 & 216 & 186 & 204 & 142 & 182 & 160 & 210 & 208 & 170\end{array}$ $\begin{array}{llllllllllll}130615 A & 1970 & 152 & 118 & 188 & 192 & 282 & 264 & 322 & 322 & 320 & 236\end{array}$

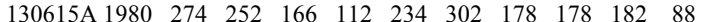
$\begin{array}{llllllllllll}130615 \mathrm{~A} & 1990 & 124 & 98 & 184 & 210 & 264 & 188 & 160 & 170 & 260 & 184\end{array}$ 130615A $2000 \quad 110 \quad 68 \quad 104-9999$

130615B 1929472

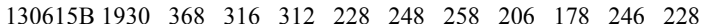
$\begin{array}{llllllllllll}130615 B & 1940 & 208 & 172 & 200 & 200 & 156 & 128 & 216 & 234 & 162 & 136\end{array}$

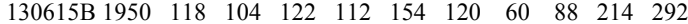
$\begin{array}{lllllllllll}130615 \mathrm{~B} 1960 & 264 & 332 & 286 & 124 & 98 & 184 & 246 & 234 & 270 & 154\end{array}$ 130615B $1970 \begin{array}{lllllllllll}230 & 178 & 116 & 246 & 140 & 154 & 142 & 194 & 170 & 264\end{array}$

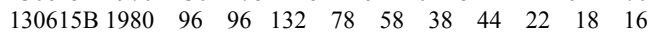

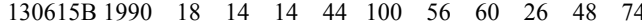
130615B $2000 \quad 34 \quad 136 \quad 46-9999$

130616A 1919160
130616A 1920 $260 \begin{array}{llllllllll}262 & 250 & 306 & 254 & 184 & 162 & 178 & 238 & 282\end{array}$ $\begin{array}{lllllllllll}130616 \text { A } 1930 & 300 & 294 & 378 & 400 & 392 & 472 & 342 & 404 & 356 & 348\end{array}$

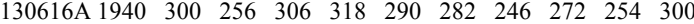
$\begin{array}{lllllllllll}130616 \text { A } 1950 & 260 & 242 & 220 & 224 & 228 & 238 & 238 & 288 & 272 & 256\end{array}$ $\begin{array}{lllllllllll}130616 \text { A } 1960 & 280 & 332 & 328 & 294 & 296 & 196 & 184 & 208 & 212 & 244\end{array}$ $\begin{array}{lllllllllll}130616 \text { A } 1970 & 302 & 210 & 224 & 214 & 184 & 206 & 190 & 158 & 208 & 114\end{array}$ 130616A $1980178 \begin{array}{llllllllll}114 & 138 & 114 & 122 & 158 & 154 & 150 & 110 & 134\end{array}$

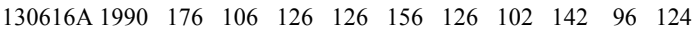
130616A $2000 \quad 106 \quad 130 \quad 120-9999$

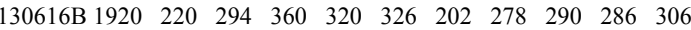

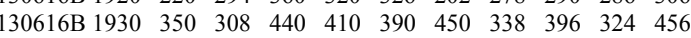

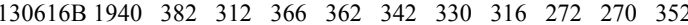

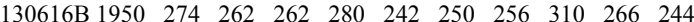

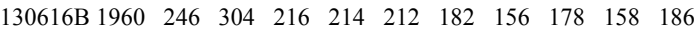
$\begin{array}{lllllllllll}130616 \mathrm{~B} 1970 & 220 & 196 & 190 & 178 & 212 & 206 & 178 & 164 & 192 & 136\end{array}$

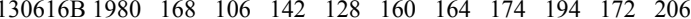

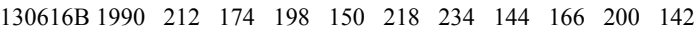
130616B $2000 \quad 212 \quad 246 \quad 160-9999$

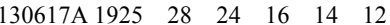

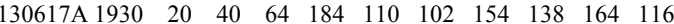
$\begin{array}{lllllllllll}130617 \text { A } 1940 & 134 & 148 & 194 & 154 & 108 & 114 & 134 & 34 & 102 & 58\end{array}$

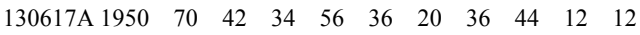
$\begin{array}{lllllllllll}130617 \mathrm{~A} 1960 & 20 & 24 & 24 & 56 & 58 & 84 & 140 & 124 & 72 & 78\end{array}$ $\begin{array}{lllllllllll}130617 \mathrm{~A} 1970 & 84 & 100 & 152 & 106 & 100 & 82 & 110 & 106 & 50 & 48\end{array}$ $\begin{array}{lllllllllll}130617 \mathrm{~A} 1980 & 48 & 34 & 18 & 42 & 48 & 72 & 60 & 60 & 68 & 58\end{array}$ $\begin{array}{lllllllllll}130617 \mathrm{~A} 1990 & 40 & 22 & 18 & 18 & 16 & 20 & 14 & 20 & 88 & 134\end{array}$ 130617A $2000 \quad 114 \quad 134 \quad 136-9999$

130617B $1938 \quad 82 \quad 192$

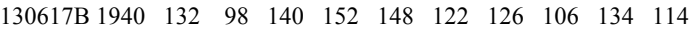

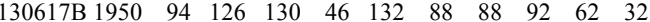

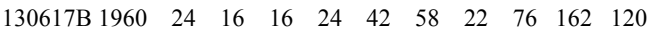

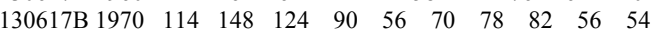

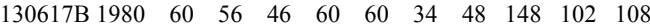

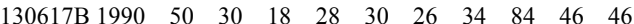
130617B $2000 \quad 30 \quad 32 \quad 38-9999$ 130618A $1957 \quad 150 \quad 88 \quad 62$

$\begin{array}{lllllllllll}130618 \mathrm{~A} 1960 & 46 & 58 & 40 & 34 & 28 & 34 & 32 & 34 & 46 & 112\end{array}$ $\begin{array}{lllllllllll}130618 \mathrm{~A} 1970 & 46 & 84 & 10 & 128 & 58 & 86 & 46 & 78 & 32 & 34\end{array}$ $\begin{array}{lllllllllll}130618 \mathrm{~A} 1980 & 42 & 42 & 44 & 40 & 28 & 70 & 38 & 44 & 42 & 40\end{array}$

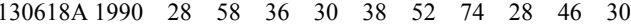
130618 A $2000 \quad 26 \quad 26 \quad 34-9999$

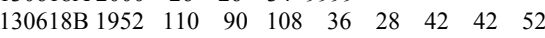

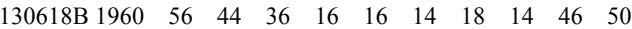
$\begin{array}{lllllllllll}130618 \mathrm{~B} 1970 & 40 & 28 & 44 & 66 & 52 & 64 & 42 & 90 & 88 & 74\end{array}$ $\begin{array}{lllllllllll}130618 \mathrm{~B} 1980 & 62 & 60 & 60 & 114 & 112 & 46 & 82 & 132 & 34 & 48\end{array}$

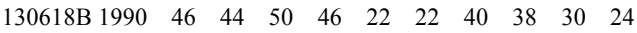
130618B $2000 \quad 22 \quad 20 \quad 38-9999$

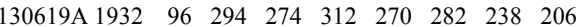

$\begin{array}{lllllllllll}130619 \text { A } 1940 & 232 & 248 & 258 & 224 & 206 & 244 & 230 & 192 & 194 & 214\end{array}$

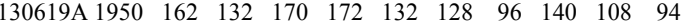

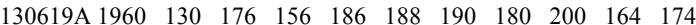

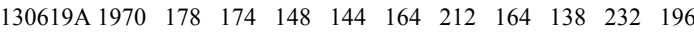
$\begin{array}{lllllllllll}130619 \text { A } 1980 & 222 & 198 & 238 & 240 & 276 & 254 & 242 & 154 & 150 & 210\end{array}$

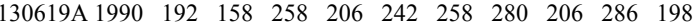
130619A $2000 \quad 270 \quad 360 \quad 210-9999$

130619B $1937 \quad 332 \quad 268 \quad 236$

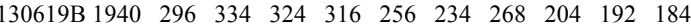

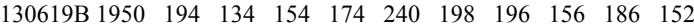

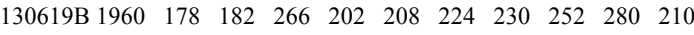

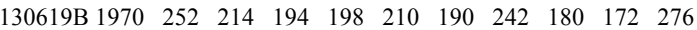

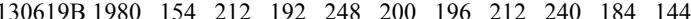
130619B 1990 $186 \begin{array}{llllllllll}196 & 156 & 232 & 224 & 214 & 234 & 212 & 198 & 178\end{array}$ 130619B 2000 $136 \quad 186 \quad 142-9999$

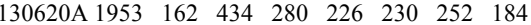

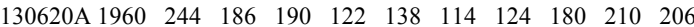
$\begin{array}{lllllllllll}130620 \mathrm{~A} 1970 & 200 & 206 & 166 & 174 & 112 & 122 & 104 & 116 & 118 & 138\end{array}$ $\begin{array}{lllllllllll}130620 \text { A } 1980 & 114 & 128 & 120 & 138 & 138 & 162 & 124 & 184 & 190 & 158\end{array}$

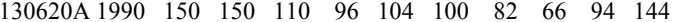
130620A $2000 \quad 122 \quad 106 \quad 132-9999$ 130620B $1948 \quad 214 \quad 138$

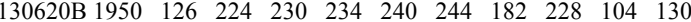
$\begin{array}{lllllllllll}\text { 130620B } 1960 & 128 & 110 & 102 & 104 & 116 & 134 & 138 & 146 & 148 & 118\end{array}$

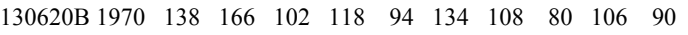

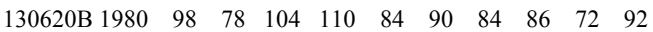

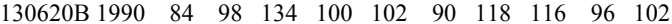
130620B 2000 $124 \quad 130 \quad 96-9999$ $\begin{array}{llllllllll}130621 \mathrm{~A} 1971 & 206 & 194 & 222 & 348 & 238 & 180 & 152 & 126 & 84\end{array}$

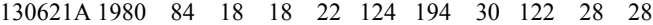
$\begin{array}{lllllllllll}130621 \mathrm{~A} 1990 & 26 & 26 & 68 & 26 & 24 & 20 & 16 & 10 & 14 & 18\end{array}$ 130621A $2000 \quad 20 \quad 42 \quad 40-9999$ 


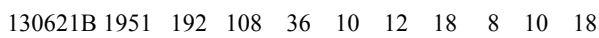

$\begin{array}{lllllllllll}130621 \mathrm{~B} 1960 & 24 & 44 & 56 & 82 & 112 & 192 & 138 & 194 & 126 & 184\end{array}$

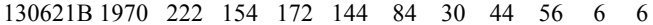

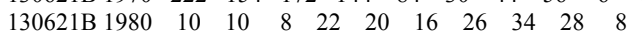

$\begin{array}{lllllllllll}130621 \mathrm{~B} 1990 & 18 & 28 & 24 & 10 & 8 & 10 & 4 & 28 & 24 & 44\end{array}$

130621B $2000 \quad 28 \quad 42 \quad 32-9999$

*Increment core identification code consists of watershed (07 or 13$)$, plot (01 to 06$)$, tree number, then core number (A or B).

Measurements are listed in a decadal series and expressed in $0.01 \mathrm{~mm}$. 


\section{VITAE}

Nicholas R. Doner

\section{EDUCATION}

M. A. in Geography, West Virginia University, 2004. Thesis: Effects of drought on the growth of young and mature temperate forests in West Virginia.

B. S. in Biology (Geography minor, History minor), West Virginia University, 2001.

Independent undergraduate research: Spatial patterns of nitrate availability in three forested watershed in the Fernow Experimental Forest.

\section{TEACHING EXPERIENCE}

Laboratory Instructor/Teaching Assistant. West Virginia University. Six sections, two semesters, 2002-2003.

\section{Courses Taught:}

Physical Geography Laboratory

\section{AWARDS AND GRANTS}

2003 Travel support money (award) to the $99^{\text {th }}$ Annual Meeting of the Association of American Geographers (\$200). West Virginia University, Eberly College of Arts and Sciences.

\section{PUBLICATIONS}

Kernan, J. T., N. Doner, H. Freeman, S. Kazar, A. E. Hessl. (in review). Can mangroves serve as indicators of global sea level change? Submitted to Southeastern Geographer, 2003.

\section{PAPER PRESENTATIONS}

N. R. Doner and A. E. Hessl. 2003. Effects of drought on the growth of young and mature temperate forests in West Virginia. Special Session at Association of American Geographers, sponsored by Biogeography Specialty Group, Annual meeting, New Orleans, LA. March 2003. 


\section{OTHER ACADEMIC EMPLOYMENT}

Department physical geography laboratory manual proofreader. Department of Geology and Geography, Eberly College of Arts and Sciences, West Virginia University. May 2003 June 2003.

Research assistant (Dr. Amy E. Hessl). Department of Geology and Geography, Eberly College of Arts and Sciences, West Virginia University. May 2002 - May 2003.

Laboratory employee (Hollowfill project). Natural Resource Analysis Center, Davis College of Agriculture, Forestry, and Consumer Sciences, West Virginia University. May 2001 May 2002.

Laboratory employee (Dr. Jonathan Cumming). Department of Biology, Eberly College of Arts and Sciences, West Virginia University. January 2001 - May 2001.

Laboratory employee (Dr. William Peterjohn and Dr. Martin Christ). Department of Biology, Eberly College of Arts and Sciences, West Virginia University. September 1999 December 2000.

\section{MEMBERSHIPS IN PROFESSIONAL SOCIETIES}

Association of American Geographers (AAG)

SouthEastern Division of the AAG
2002 to present

2002 to present 